Utah State University

DigitalCommons@USU

\title{
Analysis of Angles-Only Hybrid Space-Based/Ground-Based Approach for Geosynchronous Orbit Catalog Maintenance
}

Blythe A. Andrews

Utah State University

Follow this and additional works at: https://digitalcommons.usu.edu/etd

Part of the Aerospace Engineering Commons, and the Mechanical Engineering Commons

\section{Recommended Citation}

Andrews, Blythe A., "Analysis of Angles-Only Hybrid Space-Based/Ground-Based Approach for Geosynchronous Orbit Catalog Maintenance" (2017). All Graduate Theses and Dissertations. 6550.

https://digitalcommons.usu.edu/etd/6550

This Dissertation is brought to you for free and open access by the Graduate Studies at DigitalCommons@USU. It has been accepted for inclusion in All Graduate Theses and Dissertations by an authorized administrator of DigitalCommons@USU. For more information, please contact digitalcommons@usu.edu.

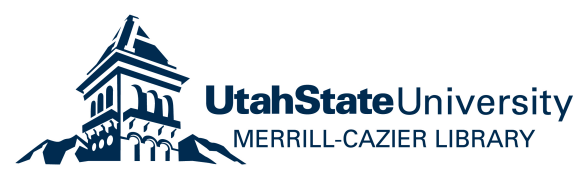


ANALYSIS OF ANGLES-ONLY HYBRID SPACE-BASED/GROUND-BASED

APPROACH FOR GEOSYNCHRONOUS ORBIT

CATALOG MAINTENANCE

by

Blythe A. Andrews

A dissertation submitted in partial fulfillment

of the requirements for the degree

of

DOCTOR OF PHILOSOPHY

in

Aerospace Engineering

Approved:

David K. Geller, Ph.D.

R. Rees Fullmer, Ph.D.

Major Professor

Committee Member

Stephen A. Whitmore, Ph.D.

James T. Wheeler, Ph.D.

Committee Member

Committee Member

Richard G. Cobb, Ph.D.

Mark R. McLellan, Ph.D.

Committee Member

Vice President for Research and

Dean of the School of Graduate Studies

UTAH STATE UNIVERSITY

Logan, Utah 
This material is declared a work of the U.S. Government and is not subject to copyright protection in the United States 
The views expressed in this dissertation are those of the author and do not reflect the official policy or position of the United States Air Force, Department of Defense, or the U.S. Government 


\author{
ABSTRACT \\ Geosynchronous Orbit Catalog Maintenance \\ by \\ Blythe A. Andrews, Doctor of Philosophy \\ Utah State University, 2017 \\ Major Professor: David K. Geller, Ph.D. \\ Department: Mechanical and Aerospace Engineering
}

Analysis of Angles-Only Hybrid Space-Based/Ground-Based Approach for

Geosynchronous Equatorial Orbit (GEO) is critical to Earth communications, weather monitoring, and national defense. Orbit estimation of GEO objects is difficult due to physical constraints placed on ground-based tracking devices such as weather, object range, and tracking frequency restrictions. These constraints are commonly mitigated through the use of two-way signaling devices for cooperative GEO satellites. However, determining the position and velocity of uncooperative GEO satellites and/or objects is more challenging. The objective of this dissertation is to quantify the increased orbital accuracy of objects in the GEO catalog when the Air Force Space Command Space Surveillance Network (AFSPC SSN) is augmented with space-based angles-only measurements from a sensor in a unique near-GEO orbit. Linear covariance theory and analysis provides an efficient method to determine the covariance of the position and velocity of an uncooperative GEO object, while incorporating uncertainties in the dynamics and sensor errors. Once this covariance is determined, an error budget analysis is performed to determine the major sources of uncertainty contributing to position errors of objects in the GEO catalog. As a result, it 
is shown through linear covariance analysis that incorporating measurements from a spacebased sensor in a near-GEO orbit increases the orbital accuracy of GEO objects when compared to the orbital accuracy achieved with AFSPC SSN measurements alone.

(250 pages) 


\title{
PUBLIC ABSTRACT
}

\author{
Analysis of Angles-Only Hybrid Space-Based/Ground-Based Approach for \\ Geosynchronous Orbit Catalog Maintenance \\ Blythe A. Andrews
}

Geosynchronous Equatorial Orbit (GEO) is critical to Earth communications, weather monitoring, and national defense. Orbit estimation of GEO objects is difficult due to physical constraints placed on ground-based tracking devices such as weather, object range, and tracking frequency restrictions. These constraints are commonly mitigated through the use of two-way signaling devices for cooperative GEO satellites. However, determining the position and velocity of uncooperative GEO satellites and/or objects is more challenging. The objective of this dissertation is to quantify the increased orbital accuracy of objects in the GEO catalog when the Air Force Space Command Space Surveillance Network (AFSPC SSN) is augmented with space-based angles-only measurements from a sensor in a unique near-GEO orbit. Linear covariance theory and analysis provides an efficient method to determine the covariance of the position and velocity of an uncooperative GEO object, while incorporating uncertainties in the dynamics and sensor errors. Once this covariance is determined, an error budget analysis is performed to determine the major sources of uncertainty contributing to position errors of objects in the GEO catalog. As a result, it is shown through linear covariance analysis that incorporating measurements from a spacebased sensor in a near-GEO orbit increases the orbital accuracy of GEO objects when compared to the orbital accuracy achieved with AFSPC SSN measurements alone. 


\section{ACKNOWLEDGMENTS}

I would like to acknowledge those that have made this work and the next level in my education possible. Through their support and guidance, the toughest times were overcome.

First, I wish to acknowledge my advisor, Dr. David Geller. His faith in me from the start of this journey made this work possible. Dr. Geller provided the guidance and mentoring required to complete this research. He is an excellent teacher, role model, professor, and friend.

Next, I wish to thank Martin France. He provided me the opportunity to pursue this degree and for that I am grateful. I'm glad I could achieve your expectation.

Lastly, this work would not of been possible without the love and support of my husband. He selflessly supported my decision to pursue this degree. He understood the challenges I faced and allowed me the time I needed to overcome them. Our daughter made this journey that much more enjoyable. I hope to be a role model for you as you grow. 


\section{CONTENTS}

\section{Page}

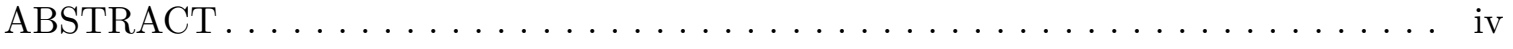

PUBLIC ABSTRACT ........................ vi

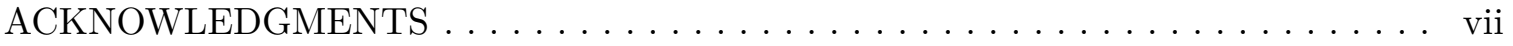

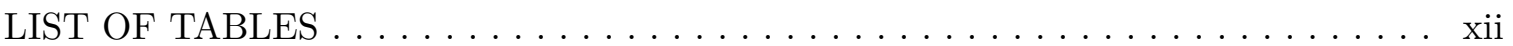

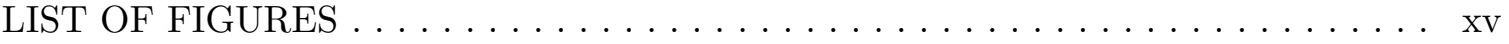

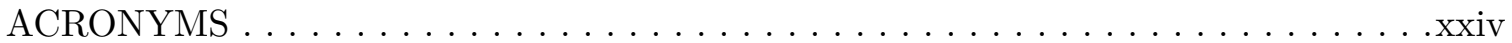

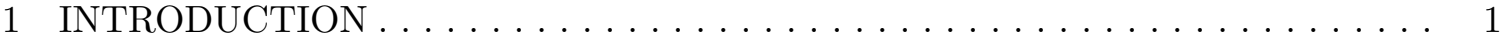

1.1 Dissertation Thesis Statement . . . . . . . . . . . . . . 2

1.2 Dissertation Overview ........................ 3

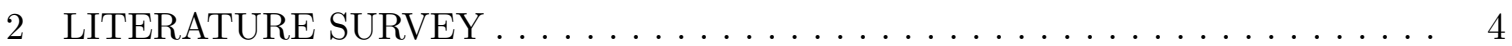

2.1 Geosynchronous Orbits . . . . . . . . . . . . . . . . . 4

2.2 Geosynchronous Angles-Only Orbit Estimation . . . . . . . . . . 5

2.2.1 Ground-Based Sensors . . . . . . . . . . . . . . . . 6

$2.2 .2 \quad$ Space-Based Sensors . . . . . . . . . . . . . . . . . 6

2.2.3 Geosynchronous Orbit Estimation . . . . . . . . . . . . . 7

2.3 Orbit Estimation Analysis Tools _. . . . . . . . . . . . . . . . 9

2.3.1 Monte Carlo Analysis . . . . . . . . . . . . . . . . . . . 10

2.3.2 Linear Covariance Analysis / Error Budget Analysis . . . . . . . . . 11

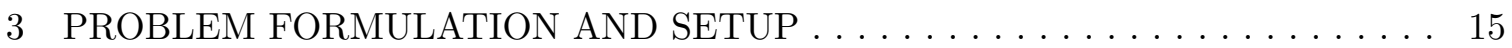

3.1 Formulation . . . . . . . . . . . . . . . . . 15

3.1.1 Resident Space Object, Space Sensor, and Ground Stations . . . . . 15

3.1 .2 Measurements . . . . . . . . . . . . . . . . 17

3.1.3 Tasking Profiles . . . . . . . . . . . . . . . . . . . . . . 19

3.2 Dynamics . . . . . . . . . . . . . . . . . . . 20

3.3 Orbit Estimation Overview and Approach . . . . . . . . . . . . 22

3.4 Summary . . . . . . . . . . . . . . . . . . . 22

4 MONTE CARLO SIMULATION-TRUTH MODELS . . . . . . . . . . . . . . 23

4.1 Nonlinear Truth Model . . . . . . . . . . . . . . . . . . . . 23

4.2 Truth Model Dynamics . . . . . . . . . . . . . . . . . 24

4.2.1 Spherical Harmonic Gravity Model . . . . . . . . . . . . . . . . 25

4.2.2 Disturbance Accelerations . . . . . . . . . . . . . . . . 26

4.2.3 Truth Model for Angle Only Measurements . . . . . . . . . . . . 26

4.2.4 Truth Model for GPS/USN Measurements . . . . . . . . . . . . 27 
4.3 Truth State Vector . . . . . . . . . . . . . . . . . . 28

4.4 Summary . . . . . . . . . . . . . . . . . . . 29

5 MONTE CARLO SIMULATION-EXTENDED KALMAN FILTER . . . . . . . . 30

5.1 Extended Kalman Filter Overview . . . . . . . . . . . . . . . . . . 30

5.2 EKF Model Dynamics . . . . . . . . . . . . . . . . . . . . . 31

5.2.1 Spherical Harmonic Gravity Model . . . . . . . . . . . . . . . 32

5.2 .2 Disturbance Accelerations . . . . . . . . . . . . . . . . . . 32

5.2.3 EKF Angles-Only Measurement Model . . . . . . . . . . . . . . . . . 32

5.2.4 EKF GPS/USN Measurement Model . . . . . . . . . . . . . . . . 33

5.3 EKF State Vector . . . . . . . . . . . . . . . . . . . 34

5.4 Covariance Propagation and the Jacobian . . . . . . . . . . . . . . 35

5.5 EKF Covariance Update . . . . . . . . . . . . . . . . . . . 38

5.6 Summary . . . . . . . . . . . . . . . . . . . . . 41

6 MONTE CARLO SIMULATION-PARAMETER VALUES . . . . . . . . . . . . . . 42

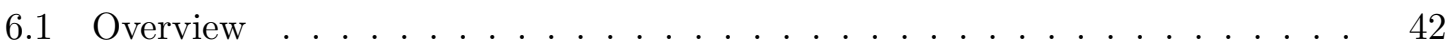

6.2 RSO and SS Initial Position and Velocity Uncertainties . . . . . . . . . . . . 42

6.3 Ground Station and SS Biases and Noise . . . . . . . . . . . . . 43

6.4 Area-to-Mass Parameters . . . . . . . . . . . . . . . . . 43

6.5 Disturbance Accelerations . . . . . . . . . . . . . . . . . 46

6.6 Random Accelerations . . . . . . . . . . . . . . . . . . 54

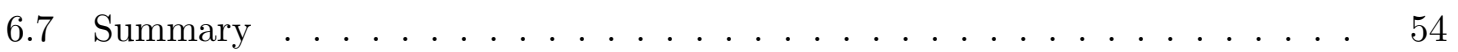

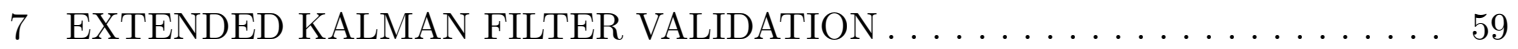

7.1 EKF Validation Method Overview . . . . . . . . . . . . . . . 59

7.2 Measurement Bias Estimation . . . . . . . . . . . . . . . 60

7.3 SRP Estimation . . . . . . . . . . . . . . . . . . . . . . 62

7.4 Disturbance Acceleration Estimation . . . . . . . . . . . . . . 64

7.5 Ground Station Only Measurement Validation . . . . . . . . . . . . 66

7.6 Space Sensor and Ground Station Measurement Validation . . . . . . . . . 70

7.6.1 Low Circular Space Sensor Orbit . . . . . . . . . . . . . . . 70

7.6.2 High Circular Space Sensor Orbit . . . . . . . . . . . . . . . 77

7.6 .3 Elliptical Space Sensor Orbit . . . . . . . . . . . . . . . . . 79

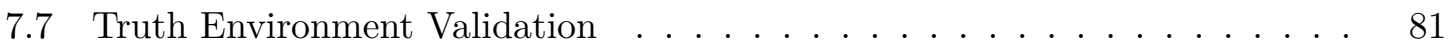

7.7.1 Ground Station Only Measurements . . . . . . . . . . . . 82

7.7.2 Low Circular Space Sensor Orbit . . . . . . . . . . . . . . . 85

7.7.3 High Circular Space Sensor Orbit . . . . . . . . . . . . . . . . . 91

7.7 .4 Elliptical Space Sensor Orbit . . . . . . . . . . . . . . . . . 92

7.8 Summary . . . . . . . . . . . . . . . . . . . . 94

8 LINEAR COVARIANCE ANALYSIS TOOL DEVELOPMENT . . . . . . . . . . 95

8.1 LinCov Overview . . . . . . . . . . . . . . . . . . . . . 95

8.2 LinCov Models . . . . . . . . . . . . . . . . . . . . . . . 98

8.2.1 LinCov Covariance Propagation . . . . . . . . . . . . . . . . . . . . 99

8.2 .2 LinCov Covariance Update . . . . . . . . . . . . . . . . . . . 102

8.3 Error Budget Analysis . . . . . . . . . . . . . . . . . . 106 
8.4 Summary . . . . . . . . . . . . . . . . . . 107

9 LINEAR COVARIANCE ANALYSIS TOOL VALIDATION . . . . . . . . . . . . 108

9.1 LinCov Validation Method Overview . . . . . . . . . . . . . . . . 108

9.2 Measurement Bias Estimation . . . . . . . . . . . . . . . . . . 108

9.3 SRP Estimation . . . . . . . . . . . . . . . . . . . . 110

9.4 Disturbance Acceleration Estimation . . . . . . . . . . . . . . . . 112

9.5 Ground Station Only Measurement Validation _. . . . . . . . . . . . 113

9.6 Space Sensor and Ground Station Measurement Validation . . . . . . . . . 117

9.6.1 Low Circular Space Sensor Orbit . . . . . . . . . . . . . . . . 117

9.6.2 High Circular Space Sensor Orbit . . . . . . . . . . . . . . . . . . . 124

9.6.3 Elliptical Space Sensor Orbit _. . . . . . . . . . . . . . 126

9.7 Truth Environment Verification . . . . . . . . . . . . . . . 128

9.7.1 Ground Station Measurements - Low Errors . . . . . . . . . . . . . . 129

9.7.2 Low Circular Space Sensor Orbit . . . . . . . . . . . . . . . . . . 132

9.7.3 High Circular Space Sensor Orbit . . . . . . . . . . . . . . . . . . . 138

9.7.4 Elliptical Space Sensor Orbit . . . . . . . . . . . . . . . 139

9.8 Summary . . . . . . . . . . . . . . . . . . . . . . . . 141

10 RESULTS - SPACE SENSOR AUGMENTATION BASELINE RESULTS . . . . . 142

10.1 Results Overview . . . . . . . . . . . . . . . . . . . . . 142

10.2 Ground Station Measurements Only . . . . . . . . . . . . . . . . 143

10.3 Low Circular Space Sensor Orbit . . . . . . . . . . . . . . . . . . . 145

10.4 High Circular Space Sensor Orbit . . . . . . . . . . . . . . . . . 148

10.5 Elliptical Space Sensor Orbit ． . . . . . . . . . . . . . . 151

10.6 Summary . . . . . . . . . . . . . . . . . . . . 154

11 RESULTS - REDUCED GROUND STATION MEASUREMENT PERIODS . . . 156

11.1 Results Overview . . . . . . . . . . . . . . . 156

11.2 Ground Station Only . . . . . . . . . . . . . . . . 156

11.3 Low Circular Space Sensor Orbit . . . . . . . . . . . . . . . . . . 158

11.4 High Circular Space Sensor Orbit . . . . . . . . . . . . . . . . . . 159

11.5 Elliptical Space Sensor Orbit . . . . . . . . . . . . . . . . 161

11.6 Summary . . . . . . . . . . . . . . . . . . . . . 162

12 RESULTS - EXTENDED SPACE SENSOR MEASUREMENT PERIODS . . . . . 164

12.1 Results Overview . . . . . . . . . . . . . . . . . . . . 164

12.2 Low Circular Space Sensor Orbit . . . . . . . . . . . . . . . . . . . 164

12.3 High Circular Space Sensor Orbit . . . . . . . . . . . . . . . . 165

12.4 Elliptical Space Sensor Orbit ～. . . . . . . . . . . . . . . 166

12.5 Summary . . . . . . . . . . . . . . . . . . 167

13 RESULTS - RSO INCLINATION ANALYSIS . . . . . . . . . . . . . . . . . . 169

13.1 Results Overview . . . . . . . . . . . . . . . . . . 169

13.2 Ground Station Only . . . . . . . . . . . . . . . . . . 169

13.3 Low Circular Space Sensor . . . . . . . . . . . . . . . . . . . . 170

13.4 High Circular Space Sensor . . . . . . . . . . . . . . . . . . . . . . . . . . 172

13.5 Elliptical Space Sensor . . . . . . . . . . . . . . . . . 176 
13.6 Summary . . . . . . . . . . . . . . . . . . . 178

14 RESULTS - SPACE SENSOR INCLINATION ANALYSIS . . . . . . . . . . . . . 180

14.1 Results Overview . . . . . . . . . . . . . . . . . . 180

14.2 Low Circular Space Sensor . . . . . . . . . . . . . . . . . . . 180

14.3 High Circular Space Sensor . . . . . . . . . . . . . . . . . . . . 181

14.4 Elliptical Space Sensor ． . . . . . . . . . . . . . . . . . . . . 184

14.5 Summary . . . . . . . . . . . . . . . . . . . 185

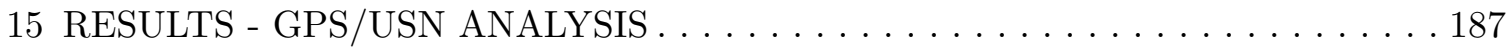

15.1 Results Overview . . . . . . . . . . . . . . . . . . . 187

15.2 GPS Accuracy . . . . . . . . . . . . . . . . . . 187

15.3 SSC USN Accuracy Analysis . . . . . . . . . . . . . . . . . . 189

15.4 Summary . . . . . . . . . . . . . . . . . . . . 193

16 RESULTS - ERROR BUDGET ANALYSIS . . . . . . . . . . . . . . . . 195

16.1 Error Budget Analysis Overview . . . . . . . . . . . . . . . . 195

16.2 Ground Station Only . . . . . . . . . . . . . . . . . 196

16.3 Low Circular Orbit Space Sensor . . . . . . . . . . . . . . . . . . 201

16.4 High Circular Space Sensor Orbit . . . . . . . . . . . . . . . . 206

16.5 Elliptical Space Sensor Orbit . . . . . . . . . . . . . . . . 210

16.6 Summary . . . . . . . . . . . . . . . . . . . . . 214

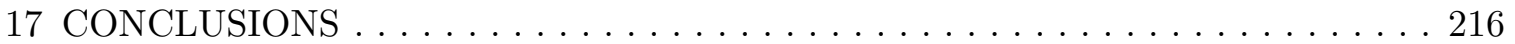

17.1 Dissertation Thesis Statement . . . . . . . . . . . . . . . 216

17.2 Summary of Results . . . . . . . . . . . . . . . . . 216

17.3 Future Work . . . . . . . . . . . . . . . . . 218

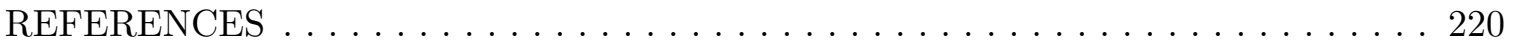

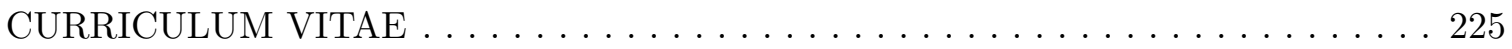




\section{LIST OF TABLES}

Table

Page

3.1 RSO and SS Orbital Elements . . . . . . . . . . . . . . . . . 16

3.2 GEODSS Ground Station Geographic Characteristics _ . . . . . . . . . 16

6.1 RSO Initial State Vector Uncertainties . . . . . . . . . . . . . . . . 55

6.2 Space Sensor Initial State Vector Uncertainties . . . . . . . . . . . . . 56

6.3 Measurement Errors _. . . . . . . . . . . . . . . . . 57

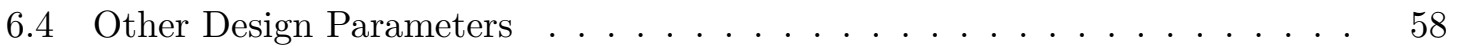

10.1 Baseline 1-week RSO Position Results, Nominal Tasking Profiles . . . . . . 155

10.2 Baseline 2-week RSO Position Results, Nominal Tasking Profiles . . . . . . 155

11.1 Number of GS Measurement Periods Analysis - Ground Station Only, 1 week results . . . . . . . . . . . . . . . . . . 157

11.2 Number of GS Measurement Periods Analysis - Ground Station Only, 2 week results . . . . . . . . . . . . . . . . . 157

11.3 Number of GS Measurement Periods Analysis - GS and Low Circular SS, 1 week results . . . . . . . . . . . . . . . . . . . 158

11.4 Number of GS Measurement Periods Analysis - GS and Low Circular SS, 2 week results . . . . . . . . . . . . . . . .

11.5 Number of GS Measurement Periods Analysis - GS and High Circular SS, 1 week results . . . . . . . . . . . . . . . . .

11.6 Number of GS Measurement Periods Analysis - GS and High Circular SS, 2 week results . . . . . . . . . . . . . . . . . . . 160

11.7 Number of GS Measurement Periods Analysis - GS and Elliptical SS, 1 week results . . . . . . . . . . . . . . . . . . . 161

11.8 Number of GS Measurement Periods Analysis - GS and Elliptical SS, 2 week

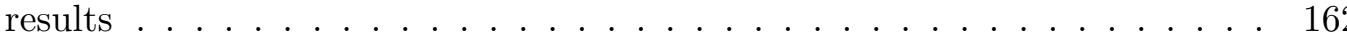


12.1 Extended SS Measurement Periods Analysis - GS and Low Circular SS . . . 165

12.2 Extended SS Measurement Periods Analysis - GS and High Circular SS . . 166

12.3 Extended SS Measurement Periods Analysis - GS and Elliptical SS . . . . . 167

13.1 RSO Inclination Analysis - Ground Station Only . . . . . . . . . . . . . 170

13.2 RSO Inclination Analysis - GS and Low Circular SS . . . . . . . . . . . 172

13.3 RSO Inclination Analysis - GS and High Circular SS . . . . . . . . . . 176

13.4 RSO Inclination Analysis - GS and Elliptical SS . . . . . . . . . . . 178

14.1 SS Inclination Analysis - GS and Low Circular SS . . . . . . . . . . . 181

14.2 SS Inclination Analysis - GS and High Circular SS . . . . . . . . . . . . 184

14.3 SS Inclination Analysis - GS and Elliptical SS . . . . . . . . . . . . . . 185

15.1 GPS Analysis - Low Circular SS Orbit, High GPS Errors . . . . . . . . . . 188

15.2 GPS Analysis - High Circular SS Orbit, High GPS Errors . . . . . . . . . . . 188

15.3 GPS Analysis - Elliptical SS Orbit, High GPS Errors . . . . . . . . . . . . 189

15.4 USN Analysis - Low Circular SS Orbit, Low Initial Errors . . . . . . . . . . . . 190

15.5 USN Analysis - High Circular SS Orbit, Low Initial Errors ～. . . . . . . . . . . 191

15.6 USN Analysis - High Circular SS Orbit, Nominal Initial Errors . . . . . . . . . . 191

15.7 USN Analysis - Elliptical SS Orbit, Low Initial Errors . . . . . . . . . . . . . . . 192

15.8 USN Analysis - Elliptical SS Orbit, Nominal Initial Errors . . . . . . . . . . . . . 192

15.9 USN Analysis - Elliptical SS Orbit, High Initial Errors . . . . . . . . . . . 193

16.1 Error Budget Analysis - Ground Station Only, Nominal Tasking, 1 week to

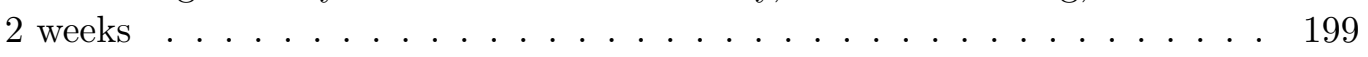

16.2 Error Budget Analysis - Ground Station Only, 2 GS Tasking, 1 week to 2 weeks . . . . . . . . . . . . . . . . . . . 201

16.3 Error Budget Analysis - Low Circular SS + GS, Nominal Tasking, 1 week to 2 weeks . . . . . . . . . . . . . . . . . 204

16.4 Error Budget Analysis - Low Circular SS + GS, 2 GS Tasking, 1 week to 2 weeks . . . . . . . . . . . . . . . . 206 
16.5 Error Budget Analysis - High Circular SS + GS, Nominal Tasking, 1 week to 2 weeks . . . . . . . . . . . . . . . . . . . 208

16.6 Error Budget Analysis - High Circular SS + GS, 2 GS Tasking, 1 week to 2 weeks . . . . . . . . . . . . . . . . . . . 210

16.7 Error Budget Analysis - Elliptical SS + GS, Nominal Tasking, 1 week to 2 weeks . . . . . . . . . . . . . . . . . . . . . 212

16.8 Error Budget Analysis - Elliptical SS + GS, 2 GS Tasking, 1 week to 2 weeks 214 


\section{LIST OF FIGURES}

Figure $\quad$ Page

3.1 Ground Station Locations and RSO Projected Starting Location (Image courtesy of Fundamentals of Astrodynamics and Applications, 4th Edition [1]) . 17

3.2 Azimuth and Elevation Measurement Geometry (Image courtesy of Orbital Mechanics for Engineering Students, 2nd Edition [2]) . . . . . . . 18

3.3 Nominal Measurement Tasking Profiles . . . . . . . . . . . . . . . . . . 20

6.1 RSO GEO orbit inertial x-component of higher-order disturbance acceleration 47

6.2 RSO GEO orbit inertial z-component of higher-order disturbance acceleration 47

6.3 RSO GEO orbit original ECRV $3-\sigma$ position error . . . . . . . . . . 48

6.4 RSO GEO orbit revised ECRV $3-\sigma$ position error . . . . . . . . . . 48

6.5 Low Circular x-component higher-order disturbance acceleration . . . . . 49

6.6 Low Circular ECRV $3-\sigma$ position error . . . . . . . . . . . . . . . 50

6.7 High Circular x-component higher-order disturbance acceleration . . . . . . 51

6.8 High Circular Original ECRV $3-\sigma$ position error . . . . . . . . . . . . 51

6.9 High Circular Revised ECRV $3-\sigma$ position error . . . . . . . . . . . . . 52

6.10 Elliptical x-component higher-order disturbance acceleration . . . . . . . 53

6.11 Elliptical ECRV $3-\sigma$ position error . . . . . . . . . . . . . . . 53

7.1 GS1 Measurement Bias, EKF Validation, Estimation Errors . . . . . . . . . 61

7.2 GS2 Measurement Bias, EKF Validation, Estimation Errors . . . . . . . . 61

7.3 Space Sensor Measurement Bias, EKF Validation, Estimation Errors . . . . 62

7.4 RSO Area-to-Mass Ratio, EKF Validation, Estimation Errors . . . . . . . 63

7.5 Space Sensor Area-to-Mass Ratio, EKF Validation, Estimation Errors . . . 64

7.6 RSO Disturbance Acceleration, EKF Validation, Estimation Errors . . . . . 65 
7.7 Low Circular Space Sensor Orbit Disturbance Acceleration, EKF Validation, Estimation Errors . . . . . . . . . . . . . . 66

7.8 Nominal Initial Errors - Ground Station Only Measurements, EKF Validation, RSO LVLH Position Estimation Errors . . . . . . . . . . . . . .

7.9 Nominal Initial Errors - Ground Station Only Measurements, EKF Validation, RSO Velocity Estimation Errors . . . . . . . . . . . . . 67

7.10 Nominal Initial Errors - Ground Station Only Measurements, EKF Validation, RSO Area-to-Mass Ratio Estimation Error . . . . . . . . . . . . . 68

7.11 Nominal Initial Errors - Ground Station Only Measurements, EKF Validation, Ground Station 1 Biases Estimation Errors _ . . . . . . . . 68

7.12 Nominal Initial Errors - Ground Station Only Measurements, EKF Validation, RSO Disturbance Accels Estimation Errors . . . . . . . . . . . .

7.13 High Initial Errors - Ground Station Only Measurements, EKF Validation, RSO Area-to-Mass Ratio Estimation Error . . . . . . . . . . . . .

7.14 Nominal Initial Errors - Ground Station and Low Circular SS Measurements, EKF Validation, RSO LVLH Position Estimation Errors . . . . . . . . . . . 71

7.15 Nominal Initial Errors - Ground Station and Low Circular SS Measurements, EKF Validation, RSO Inertial Velocity Estimation Errors . . . . . . . . . .

7.16 Nominal Initial Errors - Ground Station and Low Circular SS Measurements, EKF Validation, RSO Area-to-Mass Ratio Estimation Error . . . . . . . . .

7.17 Nominal Initial Errors - Ground Station and Low Circular SS Measurements, EKF Validation, SS LVLH Position Estimation Errors . . . . . . . . .

7.18 Nominal Initial Errors - Ground Station and Low Circular SS Measurements, EKF Validation, SS Inertial Velocity Estimation Errors . . . . . . . . . 73

7.19 Nominal Initial Errors - Ground Station and Low Circular SS Measurements, EKF Validation, SS Area-to-Mass Ratio Estimation Error . . . . . . . . .

7.20 Nominal Initial Errors - Ground Station and Low Circular SS Measurements, EKF Validation, Ground Station 1 Biases Estimation Errors . . . . . . . . .

7.21 Nominal Initial Errors - Ground Station and Low Circular SS Measurements, EKF Validation, Ground Station 2 Biases Estimation Errors . . . . . . . . .

7.22 Nominal Initial Errors - Ground Station and Low Circular SS Measurements, EKF Validation, SS Biases Estimation Errors . . . . . . . . . . . 75 
7.23 Nominal Initial Errors - Ground Station and Low Circular SS Measurements, EKF Validation, RSO Disturbance Accels Estimation Errors . . . . . . . . . 76

7.24 Nominal Initial Errors - Ground Station and Low Circular SS Measurements, EKF Validation, SS Disturbance Accels Estimation Errors . . . . . . . . . .

7.25 High Initial Errors - Ground Station and Low Circular SS Measurements, EKF Validation, RSO LVLH Position Estimation Errors . . . . . . . . . . .

7.26 Nominal Initial Errors - Ground Station and High Circular SS Measurements, EKF Validation, RSO LVLH Position Estimation Errors . . . . . . . . . . . 78

7.27 High Initial Errors - Ground Station and High Circular SS Measurements, EKF Validation, RSO LVLH Position Estimation Errors . . . . . . . . . .

7.28 Nominal Initial Errors - Ground Station and Elliptical SS Measurements, EKF Validation, RSO LVLH Position Estimation Errors . . . . . . . . . . .

7.29 High Initial Errors - Ground Station and Elliptical SS Measurements, EKF Validation, RSO LVLH Position Estimation Errors . . . . . . . . . . . .

7.30 Low Initial Errors - Ground Station Only Measurements, EKF Validation, RSO LVLH Position Estimation Errors . . . . . . . . . . . . . . . . 82

7.31 Low Initial Errors - Ground Station Only Measurements, EKF Validation, RSO Inertial Velocity Estimation Errors . . . . . . . . . . . . . .

7.32 Low Initial Errors - Ground Station Only Measurements, EKF Validation, RSO Area-to-Mass Ratio Estimation Error . . . . . . . . . . . . . . .

7.33 Low Initial Errors - Ground Station Only Measurements, EKF Validation, Ground Station 1 Biases Estimation Errors . . . . . . . . . . . . .

7.34 Low Initial Errors - Ground Station Only Measurements, EKF Validation, RSO Disturbance Accels Estimation Errors . . . . . . . . . . . . . . . . 84

7.35 Low Initial Errors - Ground Station and Low Circular SS Measurements, EKF Validation, RSO LVLH Position Estimation Errors . . . . . . . . . . .

7.36 Low Initial Errors - Ground Station and Low Circular SS Measurements, EKF Validation, RSO Inertial Velocity Estimation Errors . . . . . . . . . .

7.37 Low Initial Errors - Ground Station and Low Circular SS Measurements, EKF Validation, RSO Area-to-Mass Ratio Estimation Error . . . . . . . . .

7.38 Low Initial Errors - Ground Station and Low Circular SS Measurements, EKF Validation, SS LVLH Position Estimation Errors . . . . . . . . . . 
7.39 Low Initial Errors - Ground Station and Low Circular SS Measurements, EKF Validation, SS Inertial Velocity Estimation Errors . . . . . . . . 87

7.40 Low Initial Errors - Ground Station and Low Circular SS Measurements, EKF Validation, SS Area-to-Mass Ratio Estimation Error . . . . . . . . . .

7.41 Low Initial Errors - Ground Station and Low Circular SS Measurements, EKF Validation, Ground Station 1 Biases Estimation Errors . . . . . . . . .

7.42 Low Initial Errors - Ground Station and Low Circular SS Measurements, EKF Validation, Ground Station 2 Biases Estimation Errors . . . . . . . . . 89

7.43 Low Initial Errors - Ground Station and Low Circular SS Measurements, EKF Validation, SS Biases Estimation Errors . . . . . . . . . . .

7.44 Low Initial Errors - Ground Station and Low Circular SS Measurements, EKF Validation, RSO Disturbance Accels Estimation Errors . . . . . . . . .

7.45 Low Initial Errors - Ground Station and Low Circular SS Measurements, EKF Validation, SS Disturbance Accels Estimation Errors . . . . . . . . . .

7.46 Low Initial Errors - Ground Station and High Circular SS Measurements, EKF Validation, RSO LVLH Position Estimation Errors . . . . . . . . . . . 91

7.47 Low Initial Errors - Ground Station and High Circular SS Measurements, EKF Validation, SS Disturbance Accels Estimation Errors . . . . . . . . .

7.48 Low Initial Errors - Ground Station and Elliptical SS Measurements, EKF Validation, RSO LVLH Position Estimation Errors . . . . . . . . . . .

7.49 Low Initial Errors - Ground Station and Elliptical SS Measurements, EKF Validation, SS Disturbance Accels Estimation Errors . . . . . . . . . . . 93

9.1 GS1 Measurement Bias, LinCov Validation, Estimation Errors . . . . . 109

9.2 GS2 Measurement Bias, LinCov Validation, Estimation Errors . . . . . . 109

9.3 Space Sensor Measurement Bias, LinCov Validation, Estimation Errors . . . 110

9.4 RSO Area-to-Mass Ratio, LinCov Validation, Estimation Errors . . . . . 111

9.5 Space Sensor Area-to-Mass Ratio, LinCov Validation, Estimation Errors . . 111

9.6 RSO Disturbance Acceleration, LinCov Validation, Estimation Errors . . 112

9.7 Low Circular Space Sensor Orbit Disturbance Acceleration, LinCov Validation, Estimation Errors ． . . . . . . . . . . . . . . . . 113 
9.8 Nominal Initial Errors - Ground Station Only Measurements, LinCov Validation, RSO LVLH Position Estimation Errors . . . . . . . . . . . . . . . 114

9.9 Nominal Initial Errors - Ground Station Only Measurements, LinCov Validation, RSO Velocity Estimation Errors . . . . . . . . . . . . . .

9.10 Nominal Initial Errors - Ground Station Only Measurements, LinCov Validation, RSO Area-to-Mass Ratio Estimation Error . . . . . . . . . . . . . .

9.11 Nominal Initial Errors - Ground Station Only Measurements, LinCov Validation, Ground Station 1 Biases Estimation Errors . . . . . . . . . . . 115

9.12 Nominal Initial Errors - Ground Station Only Measurements, LinCov Validation, RSO Disturbance Accels Estimation Errors . . . . . . . . . . . .

9.13 High Initial Errors - Ground Station Only Measurements, LinCov Validation, RSO Area-to-Mass Ratio Estimation Error . . . . . . . . . . . . .

9.14 Nominal Initial Errors - Ground Station and Low Circular SS Measurements, LinCov Validation, RSO LVLH Position Estimation Errors . . . . . . . . .

9.15 Nominal Initial Errors - Ground Station and Low Circular SS Measurements, LinCov Validation, RSO Inertial Velocity Estimation Errors . . . . . . . . . 118

9.16 Nominal Initial Errors - Ground Station and Low Circular SS Measurements, LinCov Validation, RSO Area to Mass Ratio Estimation Error . . . . . . .

9.17 Nominal Initial Errors - Ground Station and Low Circular SS Measurements, LinCov Validation, SS LVLH Position Estimation Errors . . . . . . . . . . .

9.18 Nominal Initial Errors - Ground Station and Low Circular SS Measurements, LinCov Validation, SS Inertial Velocity Estimation Errors . . . . . . . . . .

9.19 Nominal Initial Errors - Ground Station and Low Circular SS Measurements, LinCov Validation, SS Area-to-Mass Ratio Estimation Error . . . . . . . . 120

9.20 Nominal Initial Errors - Ground Station and Low Circular SS Measurements, LinCov Validation, Ground Station 1 Biases Estimation Errors . . . . . . .

9.21 Nominal Initial Errors - Ground Station and Low Circular SS Measurements, LinCov Validation, Ground Station 2 Biases Estimation Errors . . . . . . .

9.22 Nominal Initial Errors - Ground Station and Low Circular SS Measurements, LinCov Validation, SS Biases Estimation Errors . . . . . . . . . . . .

9.23 Nominal Initial Errors - Ground Station and Low Circular SS Measurements, LinCov Validation, RSO Disturbance Accels Estimation Errors . . . . . . . 122 
9.24 Nominal Initial Errors - Ground Station and Low Circular SS Measurements, LinCov Validation, SS Disturbance Accels Estimation Errors . . . . . . . . 123

9.25 High Initial Errors - Ground Station and Low Circular SS Measurements, LinCov Validation, RSO LVLH Position Estimation Errors . . . . . . . . .

9.26 Nominal Initial Errors - Ground Station and High Circular SS Measurements, LinCov Validation, RSO LVLH Position Estimation Errors . . . . . . . . .

9.27 High Initial Errors - Ground Station and High Circular SS Measurements, LinCov Validation, RSO LVLH Position Estimation Errors . . . . . . . . . 126

9.28 Nominal Initial Errors - Ground Station and Elliptical SS Measurements, LinCov Validation, RSO LVLH Position Estimation Errors . . . . . . . . .

9.29 High Initial Errors - Ground Station and Elliptical SS Measurements, LinCov Validation, RSO LVLH Position Estimation Errors . . . . . . . . . . . .

9.30 Low Initial Errors - Ground Station Only Measurements, LinCov Validation, RSO LVLH Position Estimation Errors . . . . . . . . . . . . . . . . .

9.31 Low Initial Errors - Ground Station Only Measurements, LinCov Validation, RSO Inertial Velocity Estimation Errors . . . . . . . . . . . . . . . . 130

9.32 Low Initial Errors - Ground Station Only Measurements, LinCov Validation, RSO Area-to-Mass Ratio Estimation Error . . . . . . . . . . . . . . . 130

9.33 Low Initial Errors - Ground Station Only Measurements, LinCov Validation, Ground Station 1 Biases Estimation Errors . . . . . . . . . . . . . .

9.34 Low Initial Errors - Ground Station Only Measurements, LinCov Validation, RSO Disturbance Accels Estimation Errors . . . . . . . . . . . . . . .

9.35 Low Initial Errors - Ground Station and Low Circular SS Measurements, LinCov Validation, RSO LVLH Position Estimation Errors . . . . . . . . .

9.36 Low Initial Errors - Ground Station and Low Circular SS Measurements, LinCov Validation, RSO Inertial Velocity Estimation Errors . . . . . . . . .

9.37 Low Initial Errors - Ground Station and Low Circular SS Measurements, LinCov Validation, RSO Area-to-Mass Ratio Estimation Error . . . . . . .

9.38 Low Initial Errors - Ground Station and Low Circular SS Measurements, LinCov Validation, SS LVLH Position Estimation Errors . . . . . . . . . . . 134

9.39 Low Initial Errors - Ground Station and Low Circular SS Measurements, LinCov Validation, SS Inertial Velocity Estimation Errors . . . . . . . . . . 134 
9.40 Low Initial Errors - Ground Station and Low Circular SS Measurements, LinCov Validation, SS Area-to-Mass Ratio Estimation Error . . . . . . . . . 135

9.41 Low Initial Errors - Ground Station and Low Circular SS Measurements, LinCov Validation, Ground Station 1 Biases Estimation Errors . . . . . . .

9.42 Low Initial Errors - Ground Station and Low Circular SS Measurements, LinCov Validation, Ground Station 2 Biases Estimation Errors . . . . . . .

9.43 Low Initial Errors - Ground Station and Low Circular SS Measurements, LinCov Validation, SS Biases Estimation Errors . . . . . . . . . . . . 136

9.44 Low Initial Errors - Ground Station and Low Circular SS Measurements, LinCov Validation, RSO Disturbance Accels Estimation Errors . . . . . . .

9.45 Low Initial Errors - Ground Station and Low Circular SS Measurements, LinCov Validation, SS Disturbance Accels Estimation Errors . . . . . . . .

9.46 Low Initial Errors - Ground Station and High Circular SS Measurements, LinCov Validation, RSO LVLH Position Measurement Errors . . . . . . . . 138

9.47 Low Initial Errors - Ground Station and High Circular SS Measurements, LinCov Validation, SS Disturbance Accels Estimation Errors . . . . . . . . 139

9.48 Low Initial Errors - Ground Station and Elliptical SS Measurements, LinCov Validation, RSO LVLH Position Measurement Errors . . . . . . . . . . . . . 140

9.49 Low Initial Errors - Ground Station and Elliptical SS Measurements, LinCov Validation, SS Disturbance Accels Estimation Errors . . . . . . . . . . .

10.1 Baseline Results - GS Only Measurements, Nominal GS Tasking Profile, Low Initial Errors . . . . . . . . . . . . . . . . . . . . .

10.2 Baseline Results - GS Only Measurements, Nominal GS Tasking Profile, Nominal Initial Errors . . . . . . . . . . . . . . . . . . . . 144

10.3 Baseline Results - GS Only Measurements, Nominal GS Tasking Profile, High Initial Errors . . . . . . . . . . . . . . . . . .

10.4 Baseline Results - GS and Low Circular Orbit SS Measurements, Nominal Tasking Profiles, Low Initial Errors . . . . . . . . . . . . . . . . . 146

10.5 Baseline Results - GS and Low Circular Orbit SS Measurements, Nominal Tasking Profiles, Nominal Initial Errors . . . . . . . . . . . . . . . .

10.6 Baseline Results - GS and Low Circular Orbit SS Measurements, Nominal Tasking Profiles, High Initial Errors . . . . . . . . . . . . . . . . . . 148 
10.7 Baseline Results - GS and High Circular Orbit SS Measurements, Nominal Tasking Profiles, Low Initial Errors ～. . . . . . . . . . . . . . . . . 149

10.8 Baseline Results - GS and High Circular Orbit SS Measurements, Nominal Tasking Profiles, Nominal Initial Errors . . . . . . . . . . . . . . . . 150

10.9 Baseline Results - GS and High Circular Orbit SS Measurements, Nominal Tasking Profiles, High Initial Errors ． . . . . . . . . . . . . . . . . 151

10.10Baseline Results - GS and Elliptical Orbit SS Measurements, Nominal Tasking Profiles, Low Initial Errors _ . . . . . . . . . . . . . . . 152

10.11Baseline Results - GS and Elliptical Orbit SS Measurements, Nominal Tasking Profiles, Nominal Initial Errors . . . . . . . . . . . . . . . . . 153

10.12Baseline Results - GS and Elliptical Orbit SS Measurements, Nominal Tasking Profiles, High Initial Errors . . . . . . . . . . . . . . . . . . . . 154

13.1 Low Circular Space Sensor Relative Motion - RSO Inclined Analysis . . . . 171

13.2 High Circular Space Sensor Relative Motion - RSO Inclined Analysis . . . . 173

13.3 High Circular SS Orbit, Low Initial Errors, RSO Inclination Analysis - RSO $3-\sigma$ Position Error . . . . . . . . . . . . . . . . 174

13.4 High Circular SS Orbit, Low Errors, RSO Inclination Analysis - RSO 3- $\sigma$ Position Error, During SS Measurement Periods . . . . . . . . . . . . . . . 174

13.5 High Circular SS Orbit, Low Errors, RSO Inclination Analysis - RSO 3- $\sigma$ Position Error, During Second Week . . . . . . . . . . . . . . . . . 175

13.6 Elliptical Space Sensor Relative Motion - RSO Inclined Analysis . . . . . 177

14.1 SS Inclination Analysis - RSO 3- $\sigma$ Position Error, Low Initial Errors . . . . 182

14.2 SS Inclination Analysis - RSO 3- $\sigma$ Position Error, Nominal Initial Errors . . 183

16.1 Error Budget Analysis - Ground Station Only, Low Initial Errors . . . . . . 197

16.2 Error Budget Analysis - Ground Station Only, Low Initial Errors, 1 week to 2 weeks . . . . . . . . . . . . . . . . . . . 198

16.3 Error Budget Analysis - Ground Station Only, Low Initial Errors, 2 GS Meas Periods, 1 week to 2 weeks . . . . . . . . . . . . . . . 200

16.4 Error Budget Analysis - Low Circular SS + GS, Low Initial Errors . . . . . 202 
16.5 Error Budget Analysis - Low Circular SS + GS, Low Initial Errors, 1 week to 2 weeks . . . . . . . . . . . . . . . . . . 203

16.6 Error Budget Analysis - Low Circular SS + GS, Low Initial Errors, 2 GS Meas Periods, 1 week to 2 weeks . . . . . . . . . . . . . . . . 205

16.7 Error Budget Analysis - High Circular SS + GS, Low Initial Errors, 1 week to 2 weeks . . . . . . . . . . . . . . . . . 207

16.8 Error Budget Analysis - High Circular SS + GS, Low Initial Errors, 2 GS Meas Periods, 1 week to 2 weeks . . . . . . . . . . . . . . . . . 209

16.9 Error Budget Analysis - Elliptical SS + GS, Low Initial Errors, 1 week to 2 weeks . . . . . . . . . . . . . . . . . . . 211

16.10Error Budget Analysis - Elliptical SS + GS, Low Initial Errors, 2 GS Meas Periods, 1 week to 2 weeks . . . . . . . . . . . . . . . . . . . 213 
xxiv

\section{ACRONYMS}

$\begin{array}{ll}\text { AFSPC SSN } & \text { Air Force Space Command Space Surveillance Network } \\ \text { DISCOS } & \text { Database and Information System Characterising Objects in Space } \\ \text { GEO } & \text { Geosynchronous Equatorial Orbit } \\ \text { GEODSS } & \text { Ground-Based Electro-Optical Deep Space Surveillance } \\ \text { EKF } & \text { Extended Kalman Filter } \\ \text { ESA } & \text { European Space Agency } \\ \text { GSSAP } & \text { Geosynchronous Space Situational Awareness Program } \\ \text { JFCC Space } & \text { Joint Functional Component Command for Space } \\ \text { LEO } & \text { Low Earth Orbit } \\ \text { LinCov } & \text { Linear Covariance } \\ \text { RSO } & \text { Resident Space Object } \\ \text { SBSS } & \text { Space Based Space Surveillance } \\ \text { SBV } & \text { Space-Based Visible } \\ \text { SRP } & \text { Solar Radiation Pressure } \\ \text { SS } & \text { Space Sensor } \\ \text { TLE } & \text { Two-Line Element Set }\end{array}$




\section{CHAPTER 1}

\section{INTRODUCTION}

Geosynchronous Equatorial Orbit (GEO) satellites are critical to Earth communications, weather monitoring, and national defense. As a result, GEO is congested with satellites and debris. With such a congested environment, accurate tracking of GEO objects, especially uncooperative objects, is critical to the success of the operational satellites. This chapter will provide a brief overview of the research, the thesis statement of this dissertation, and finally an overview of the remaining chapters.

Orbit estimation of GEO objects is traditionally achieved through use of radar range and angle measurements (right ascension and declination). Radar range measurements are limited to very few ground-based sensors, making angles-only measurements from optical sensors the main method of tracking. The Ground-Based Electro-Optical Deep Space Surveillance (GEODSS) system, part of the Air Force Space Command Space Surveillance Network (AFSPC SSN), tracks deep space objects including GEO objects. These optical telescopes provide angles-only measurements of the objects. Ground-based, angles-only measurements for GEO satellite tracking has challenges associated with it due to physical constraints such as weather, lighting, object size detection, uncertain forces, satellite maneuvers, and other tracking restrictions. These challenges are reduced with cooperative GEO satellites through the use of two-way signaling devices, i.e. radiometric Doppler and range. However, challenges exist when tracking uncooperative objects due to the lack of two-way signaling. The addition of a near-GEO space-based angles-only sensor to assist with orbit determination is a way to overcome many of the present challenges and constraints. A space-based sensor has reduced weather constraints, better lighting conditions, has the potential to detect smaller size objects, and provides additional measurements to combine with ground-based measurements. 
Case studies exist that determine the orbit estimation accuracy achievable with multiple (ground and space-based) sensors, and in fact Low Earth Orbit (LEO) satellites are operationally used as tracking sensors, however the need exists for an efficient analysis model that can quantify the benefit of augmenting ground-based sensors with a space-based sensor. This model must also incorporate uncertainties associated with a GEO object of interest, which is referred to as a Resident Space Object (RSO). This analysis model must be able to incorporate various sensors, both ground-based and space-based, and the errors associated with these sensors. Ultimately, the uncertainty of the RSO orbit must be quantified and the sources of error contributing to the uncertainty determined.

Linear covariance (LinCov) theory and analysis is used to quantify the effect of augmenting the AFSPC SSN with angles-only measurements from near-GEO satellites. The traditional method of accomplishing this is through Monte Carlo analysis. However, Monte Carlo analysis is time consuming and laborious. In order to obtain results in an efficient manner, LinCov theory and analysis is used to assess the effect of adding the space-based measurements. Using LinCov analysis the covariance of the position and velocity of an uncooperative GEO object is determined, while incorporating uncertainties in the dynamics and sensor errors. Once this covariance is determined, an error budget analysis is performed to determine the major sources contributing to the error in position of objects in the geosynchronous catalog.

Two limitations exist with the developed LinCov analysis tool: 1) The solar radiation pressure (SRP) model is based on a modified cannonball model and is thus not applicable to, for example, high area to mass ratio (HAMR) objects, and 2) the range of initial position uncertainties for the GEO objects is limited to approximately $20 \mathrm{~km} \mathrm{1-sigma.} \mathrm{Beyond} \mathrm{this}$ level of initial position uncertainty, the developed extended Kalman filter exhibited some divergence. This is discussed further in the Chapter 6 of the dissertation.

\subsection{Dissertation Thesis Statement}

The thesis of the dissertation is that through linear covariance theory the increased orbital accuracy of objects in the GEO catalog, achievable when the AFSPC SSN is aug- 
mented with space-based angles-only measurements from a sensor in a near-GEO orbit, can be quantified.

\subsection{Dissertation Overview}

The dissertation begins, in Chapter 2, with a comprehensive literature survey relevant to the research at hand. Next, in Chapter 3, the research problem and approach is formally introduced. Chapters 4 through 6 present the development of a Monte Carlo simulation, which includes a truth model and Extended Kalman filter (EKF). Chapter 7 provides the validation of the developed EKF. Chapter 8 presents the development of the LinCov analysis tool, which builds on the developed truth model and EKF. The LinCov tool is validated in

Chapter 9. Chapters 10 through 16 present the analysis results produced through use of the LinCov tool. Finally, Chapter 17 summarizes the work performed and includes ideas for future work. 
CHAPTER 2

LITERATURE SURVEY

\subsection{Geosynchronous Orbits}

Geosynchronous orbits have the unique characteristic of possessing the same angular rate as Earth's rotation. Geosynchronous covers all orbits with a radius of approximately 42,164 km, which have the same rotational rate as Earth independent of inclination. Due to its unique rotational rate, orbits of this type with small inclinations are popular for communication and weather satellites. This research will focus on geosynchronous orbits of inclinations less than or equal to 15 degrees.

The United States Air Force Space Command Space Surveillance Network (AFSPC $\mathrm{SSN}$ ) is a system of sensors dedicated to tracking space objects. The Joint Functional Component Command for Space (JFCC Space) publishes two line element data sets for the publicly releasable tracked satellites on www.space-track.org. There are currently 808 geosynchronous satellites with available two line element data sets on the space track website $[3]$.

The European Space Agency (ESA) Operations Centre through use of their Database and Information System Characterising Objects in Space (DISCOS) Database classifies all objects near the geostationary ring into seven different types of categories:

$\mathrm{C} 1$ : objects under longitude and inclination control - longitude is nearly constant and inclination is smaller than 0.3 degrees

C2: objects under longitude control only - inclination higher than 0.3 degrees

D: objects in a drift orbit

L1: objects in a libration orbit around the Eastern stable point (75 degrees East)

L2: objects in a libration orbit around the Western stable point (105 degrees West)

L3: objects in a libration orbit around both stable points 
I: objects in a highly inclined orbit (larger than 25 degrees)

All of these objects have an eccentricity smaller than 0.2 and a semi-major axis between 39,664 and 45,314 km. All inclinations are lower than 70 degrees.

In their status report for all geosynchronous objects as of the end of 2012 , there are 1369 total objects near the geostationary ring; this includes both controlled and uncontrolled objects. By classification, the numbers break down as:

C1: 289 objects

C2: 133 objects

D: 662 objects

L1: 114 objects

L2: 46 objects

L3: 18 objects

I: 9 objects

20 objects could not be classified and 73 are uncatalogued objects [4].

An uncooperative GEO object or RSO, for purposes of this research, has an inclination less than or equal to 15 degrees and is either controlled or uncontrolled. These objects fall into all categories listed by the ESA except I, the highly inclined orbit. Uncooperative refers to an object without two-way signaling capability with the AFSPC SSN. Cooperative refers to an object with two-way signaling capability with AFSPC SSN.

\subsection{Geosynchronous Angles-Only Orbit Estimation}

Uncooperative GEO objects are typically tracked using angles-only measurements from ground-based optical telescopes. These angular measurements are right ascension and declination of the RSO. Upon gaining these measurements from a telescope, an estimate of the RSO's state (position and velocity vectors) is determined. Challenges exist that make obtaining the required angles-only measurements difficult. Ground tracking sites are limited in their capabilities through tasking profiles, lighting conditions, measurement geometry, and weather. Space-based optical telescopes provide an opportunity to overcome some of these constraints, such as weather and reduced tracking opportunities, to provide right ascension 
and declination measurements of the RSO. Relevant research in these areas is summarized below.

\subsubsection{Ground-Based Sensors}

David A. Vallado developed a comprehensive document on simulating space surveillance networks [5]. It contains a detailed list of the sensors that AFSPC SSN is comprised of which includes the location, latitude, and longitude of the ground-based optical sensor. Not only is the location of the sensor important, but realistically scheduling the sensor to track the object is important as well. A geostationary object is designed to hover over a certain spot on Earth at all times. So, for instance, if the ground-based sensor has visibility of the RSO, then technically the ground-based sensor has access to the RSO at all times and can take continuous measurements. However, taking continuous measurements is unrealistic as a certain number of constraints exist that make it impossible. Lighting conditions and Sun and Moon exclusion zones are a few of the physical constraints placed on the system. Along with the physical constraints, there are unknown factors that must be considered, such as a missed track due to weather or over-scheduling. Vallado incorporates these unknowns as a missed track probability factor. He develops tasking lists that give each sensor a pass length, observation step size, revisit time, and missed track probability.

Objects of highest importance to AFSPC are known as Category 1 objects. For category 1 objects, AFSPC needs data that day and with a high success rate. The Ground-based Electro-Optical Deep Space Surveillance (GEODSS) system which is part of the AFSPC SSN, averages around 30 percent response rate for category 1 taskings, mainly due to nighttime viewing restrictions and weather [6]. There are three operational GEODSS sites: Socorro, New Mexico; Diego Garcia, British Indian Ocean Territory; and Maui, Hawaii [7].

The field of view of these telescopes is $1.23 \times 1.61$ degrees, which is approximately $860 \mathrm{x}$ $1125 \mathrm{~km}$ at geosynchronous range [8].

\subsubsection{Space-Based Sensors}

The Space-Based Visible (SBV) Sensor is a sensor on the Midcourse Space Experiment 
satellite, which was launched in 1996 [9]. First, SBV went through an experimental demonstration phase for 18 months, after which it was incorporated as a sensor in the AFSPC SSN. The sensor observed targets required by AFSPC, just as a ground-based sensor does. SBV had the ability to cover the entire geosynchronous belt from its $900 \mathrm{~km}$ sun-synchronous orbit. This added coverage of an SSN gap that exists in the eastern hemisphere of the geosynchronous belt. One of the large contributions SBV made is that the average age of a RSOs element set was reduced by 20 percent. SBV was shown to produce more accurate observations than a GEODSS sensor.

SBV was useful in generating observations that resulted in highly accurate position estimates of geosynchronous satellites [6]. SBV made a significant contribution by detecting and classifying uncorrelated targets. These are detected objects that do not correlate with any known objects in the catalog. SBV was at or above a 90 percent response rate for category 1 taskings.

The United States Air Force launched the Space Based Space Surveillance (SBSS) satellite in 2010 [10]. This satellite serves as a follow-on to SBV. It is in a $630 \mathrm{~km}$ altitude, sun-synchronous orbit. It is capable of monitoring objects as small as 1-meter at geostationary orbit.

The United States Air Force also has a Geosynchronous Space Situational Awareness Program (GSSAP), which so far has placed four satellites in a near-geosynchronous orbit to perform as a SSN sensor [11]. These satellites also have the capability to perform Rendezvous and Proximity Operations, which allow for better surveillance. These satellites will enhance the knowledge of the geostationary orbit environment while aiding in satellite collision avoidance and will allow for more accurate tracking and characterization of man-made orbiting objects.

\subsubsection{Geosynchronous Orbit Estimation}

The risk of collision in the congested GEO environment has sparked studies and research focused on increasing the accuracy of GEO object estimation results. In particular, for those objects that do not have owner provided data, such as debris or uncooperative satellites. 
The details and results of a selection of these studies and research are provided in this section.

David Vallado has worked with others on geosynchronous orbit estimation studies [12]. One such study was performed with Jonathan Lowe and Joseph Anderson evaluating the orbit estimation benefits of placing an optical payload on a commercial satellite in geosynchronous orbit. Trade studies were performed using one or two hosted payload observations as well as ground station observations. 10-15 observations were assumed per track with an average of 4 tracks per day per sensor. The trade studies were performed using AGI ODTK and STK and measurements were simulated for each RSO from each sensor. The results show that position uncertainties for four particular geosynchronous satellites are reduced by one to two orders of magnitude when a hosted payload is used in conjunction with ground station measurements, versus just using ground station measurements. It was shown that position uncertainties under $1 \mathrm{~km}$ are possible if two hosted payloads are used and their measurements are fused with ground station measurements. It was also found that an imaging density of 10 observations per track and 6 tracks per day from one hosted payload provides an adequate orbit determination accuracy. Finally, it was determined that a single optical payload in GEO is capable of detecting as much as $45 \%$ of all GEO RSOs.

Jonathan Lowe, working with David Vallado and Bob Hall, published results of a similar trade study using five RSOs in various orbits, tracked with several ground-based and hosted payload sensors [13]. The baseline configuration consisted of two ground stations and use of two hosted payloads. Each ground station received 1 pass per day, each 3 minutes long, making angular measurements every 30 seconds. The hosted payloads took measurements every 60 seconds during a pass. The hosted payload tracks the RSO during the entire visibility window, and a pass is close to 12 hours long. The results of the baseline study varied from $110.1 \mathrm{~m}$ to $384.6 \mathrm{~m}$ two-sigma RSS error for the five RSOs. This baseline study placed a 10 arcsec white noise sigma on the hosted optical sensor measurements and 0.5 arcsec white noise sigma on the ground station measurements. 
Ray Byrne, et al. from Sandia National Laboratories analyzed the benefit of a geosynchronous observation point for orbit determination [14]. For this study, an Extended Kalman Filter was used to estimate the state of a simulated geosynchronous Tracking and Data Relay Satellite 8 orbit. Monte Carlo simulations were used to determine the RMS position error (1-sigma) based on a variety of trades. One trade study performed used two ground-based angles-only sensors and one space-based angles-only sensor. The groundbased sensor had a white noise sigma of 0.003 degrees applied and the space-based sensor had a white noise sigma of 0.003 degrees applied. The initial uncertainty surrounding the position of the RSO was $20 \mathrm{~km}$ (1-sigma) and the uncertainty surrounding the velocity was

5 percent of the initial velocity (1-sigma). This Monte Carlo study resulted in a mean RMS position error (1-sigma) of $4.494 \mathrm{~km}$ and a mean RMS velocity error (1-sigma) of 0.534 $\mathrm{m} / \mathrm{s}$. This study used only the two-body equations of motion with no noise placed into the dynamics to account for unknown forces such as solar radiation pressure.

\subsection{Orbit Estimation Analysis Tools}

The position and velocity covariance is an important piece of information gained from the orbit estimation process. It conveys the uncertainty of the estimate of the state, the variables of interest in the estimation problem. Mathematical models are used to generate both the estimate of the state as well as the covariance of that estimate. However, no deterministic mathematical model is perfect, as it does not account for all the uncertainties that exist within the model. Some forces that effect the mathematical model cannot be modeled deterministically. Finally, measurements are never perfect.

Rather than taking a deterministic mathematical modeling approach, orbit estimation is performed through stochastic modeling. Stochastic modeling allows for uncertainties in the system to be modeled and incorporated into the estimate and covariance of the state. A form of this type of stochastic model is to have a linear dynamic model of the system driven by white Gaussian noise and also to have a measurement model that is driven by white Gaussian noise. White Gaussian noise is completely described by its mean value and standard deviation [15]. In terms of this research, the uncertainties in the dynamic model 
that can be driven by white Gaussian noise are the errors in the Solar Radiation Pressure model and other unknown or mismodeled accelerations. Uncertainties in the measurement model are represented by noise and bias. The white noise is often used to drive exponentially random variables and random walks. Two available methods to analyze the effects of the uncertainties will be discussed in this section, Monte Carlo analysis and LinCov analysis.

\subsubsection{Monte Carlo Analysis}

Monte Carlo analysis consists of doing multiple analysis runs of a linear or non-linear stochastic system. Each time an analysis is performed, a different value of the white Gaussian noise is utilized that fits within its statistical description and the initial conditions of the state are created with a random number generator that fits within the starting covariance. Therefore, a different state estimate and filter covariance is obtained each time the analysis is performed. Hundreds if not thousands of these analysis runs can be performed to determine the statistics of the results of the analysis. In the end, a mean and covariance is obtained for the estimate that incorporates the uncertainties in the model. This process is time consuming and laborious, however if enough runs are performed, it is a very accurate method of achieving the results. For orbit determination, this can be used to analyze the results of a Kalman filter that is initialized with the mean value of RSO position and velocity and its associated covariance. This Kalman filter requires simulated sensor measurements to be processed as a part of the analysis, which again is time consuming to generate.

An example of an orbit determination Monte Carlo analysis was performed by Bhaskaran et al. [16], this Monte Carlo analysis was performed to assess the Deep Space 1 Autonomous Navigation System filter. 100 Monte Carlo samples of the truth model with uncertainties represented by the stochastic model were conducted. The measurements that were generated by the stochastic model were then passed to a linear least squares filter and an estimate of the state was calculated. The truth values from the stochastic model and the filter values of the state were differenced which allowed an error covariance to be developed. The error covariance was then compared to the filter covariance, which allowed the filter performance to be assessed. 


\subsubsection{Linear Covariance Analysis / Error Budget Analysis}

LinCov analysis achieves nearly the same covariance results as Monte Carlo analysis, however with the benefit of only performing one analysis run instead of hundreds or thousands. As the name implies, LinCov analysis is performed on linear systems. If the system being analyzed is non-linear, it can be linearized around a nominal trajectory prior to using the LinCov analysis method. Care must be taken to ensure the non-linear system can be approximated as linear in the region that is being analyzed. In terms of orbital dynamics, the system is non-linear, however, variations are small when compared to a nominal reference trajectory, and therefore linearizing the system is generally acceptable. The result of LinCov analysis is only the covariance, it does not provide a mean estimate of the state. It is able to provide statistical bounds on the state elements.

A benefit of Linear Covariance Analysis is that no simulated measurements are required to be generated. Instead, only the geometry of the state elements, as a result of the dynamics of the problem, are incorporated.

Once the model for the LinCov analysis is verified, it can be used to perform an error budget analysis. This analysis looks at the contribution of each individual error source present in the stochastic model and determines its contribution to the overall covariance of a particular state element of importance. This can allow further analyses into the large error sources to see if it is possible to reduce their contribution, which would reduce the overall covariance.

A rigorous linear covariance analysis and error budget method is provided by Maybeck [15] and Christensen and Geller [17]. The first goal of Maybeck's method is to verify that a developed Kalman filter produces accurate results. The foundation of Maybeck's approach is to develop a truth model that represents as closely as possible, the real world. This truth model must incorporate all critical states to the estimation problem, and be driven by white Gaussian noise. The truth model then generates measurements within the measurement process, that can have a bias and/or white Gaussian noise added to them or more complex error models that are driven by white Gaussian noise can be developed. 
These measurements are then fed to the developed Kalman filter, and in turn are processed by the Kalman filter. The truth model provides a true state, while the Kalman filter output is a state estimate. The error committed by the Kalman filter can then be calculated by differencing the true state and the state estimate. Many error samples can be generated from repeating this process. This is also known as Monte Carlo analysis, which was described previously. From the many error samples, a covariance can be calculated. The resulting error covariance is then compared to the Kalman filter's covariance. These should align closely, which shows that the Kalman filter is able to represent the error surrounding its state estimate accurately.

With a verified accurate portrayal of the real world and an associated Kalman filter, the entire problem can now be linearized around a reference trajectory. Now, with one run of the linearized model, a covariance is generated that should closely align with the Monte Carlo results. If the results align, a LinCov analysis tool is verified and is capable of generating a covariance representative of the Monte Carlo analysis runs, but in just one run.

Once this LinCov analysis tool is developed, it can be used to perform an error budget, which is also discussed in Maybeck [15] and Christensen and Geller [17]. This consists of performing a LinCov analysis run with only one source of error or a small group of error sources turned on. As a result, the effect of this one source of error is calculated. This can be done for each error source, individually. The outcome of this process is the ability to determine the most influential error sources, as well as the least influential. This can possibly allow for a reduced-state filter, by removing the least influential error sources. It can also lead to focused improvements to the most influential error sources.

In literature, various meanings and methods of LinCov analysis that differ from the above approach, used for orbit estimation purposes, can be found. There is also literature that utilizes the model of the LinCov analysis to perform an error budget analysis. Examples of these are given below.

A very basic meaning of LinCov analysis is to take a stochastic system model and 
run different measurement types/tasking scenarios on that model to see the affect on the resulting covariance. Ryan S. Park, et al. did just this by studying the resulting covariance of the Cassini spacecraft Titan flyby, where the affects of synthetic aperture radar and altimetry data types were analyzed [18].

Estefan and Folkner performed an analysis to determine how sensitive spacecraft navigation errors were to Earth Orientation calibrations [19]. A LinCov analysis was utilized and as a result error budget charts were developed that showed how much each of the principal error sources present in the model contribute to the total Mars Pathfinder orbit determination uncertainty.

Estefan and Burkhart analyzed a Mars Observer interplanetary cruise scenario for cases where different data types were utilized [20]. For their research, the linear filter model was assumed to be optimal, meaning the truth model and filter model are assumed to be the same. Error budget analysis was performed for the cases when different data types were present in the filter (Doppler, ranging, and a combined set of the two).

Hicks and Wiesel used a method similar to that of Maybeck to verify their system model [21]. Monte Carlo simulations were conducted and the filter results were then compared to the truth model. The filter was then tuned to acceptable performance before being used in the next area of their research.

Tombasco and Axelrad analyze the state error covariance of one geosynchronous object relative to another when processing space-based angles-only measurements [22]. The approach taken to process the measurements is batch processing which includes no process noise. There is noise placed on the measurements. The covariance for the along-track separation and the intersatellite range distance are analyzed. First, an analytical covariance prediction tool is developed based on a linearized two-body relative motion model. The analytical covariance developed is then verified by studying the linearization error when compared to a batch estimated uncertainty. A Monte Carlo analysis is then performed to analyze how well the variance represents the actual estimation error distribution. The Monte Carlo analysis performed 100 runs of the batch processor, where the measurement 
noise and the a priori state were randomized based on the uncertainty bounds. The analytic covariance is shown as useful to approximate the amount of tracking required to meet a desired accuracy, or the accuracy given a certain amount of tracking. Future work is noted as working to include dynamic uncertainty, such as solar radiation pressure.

In addition to Tombasco's analytical covariance work, Tombasco in her PhD dissertation states that follow-on work could include an analysis of geosynchronous estimation accuracy using sequential estimation, which would allow for process noise [23]. She states that the uncertainty modeling could be modified to include luni-solar gravity, solar radiation pressure, and higher-order Earth gravity model, and that "It is of particular interest to inspect how errors in the solar radiation pressure model affect the orbit estimation accuracy". Finally, she states that expansion to a general analytic covariance model that can incorporate multiple measurement sources (ground-based and space-based) would be useful for geosynchronous orbit estimation purposes, in addition to the case studies that exist. 
CHAPTER 3

\section{PROBLEM FORMULATION AND SETUP}

\subsection{Formulation}

To test the thesis statement of this research, a LinCov analysis tool using LinCov theory, was ultimately created that incorporates an RSO, AFSPC SSN ground stations, and a space-based sensor. This LinCov analysis tool estimates the position and velocity covariance, while incorporating the uncertainties that exist in the dynamics of the RSO and space sensor, and sensor errors. Below details the characteristics of the RSO and space sensor orbits, the ground station and space sensor tasking profiles, an overview of the dynamics incorporated, the measurements, and the orbit estimation/modeling approach taken.

\subsubsection{Resident Space Object, Space Sensor, and Ground Stations}

A generic RSO in geosynchronous orbit was chosen for this analysis. On analyzing the TLEs of all geosynchronous objects in the catalog, it can be seen that the average GEO

inclination is .05 degrees and the average eccentricity is $5 \times 10^{-4}$. Three different space sensor orbits are used in this analysis. This allows the levels of achievable accuracy based on distance from GEO to be determined, as well as to view the effects of using a circular versus elliptical orbit. A circular orbit 1,000 km below geosynchronous, a circular orbit 7,342 km below geosynchronous, and an elliptical orbit with apogee of the high circular orbit radius and perigee of the low circular orbit radius are used. The orbital elements used to initialize the RSO and space sensor for each orbit are provided in Table 3.1. 
Table 3.1: RSO and SS Orbital Elements

\begin{tabular}{|c|c|c|c|c|}
\hline Orbital Elements & RSO & Low Circular SS & High Circular SS & Elliptical SS \\
\hline Semi-major axis $(\mathrm{m})$ & 42164000 & 34822000 & 41164000 & 37993000 \\
\hline Eccentricity & $5 \times 10^{-4}$ & $1 \times 10^{-10}$ & $1 \times 10^{-10}$ & 0.0835 \\
\hline Inclination (rad) & 0.0009 & $1 \times 10^{-10}$ & $1 \times 10^{-10}$ & $1 \times 10^{-10}$ \\
\hline Right Ascension (rad) & 0.1745 & 0.2094 & 0.2094 & 0.2094 \\
\hline Arg. of Perigee (rad) & 4.1888 & 4.1888 & 4.1888 & 2.6354 \\
\hline True Anomaly (rad) & 0 & 5.7612 & 5.5335 & 4.1861 \\
\hline
\end{tabular}

Two GEODSS ground station locations are simulated for analysis: Socorro, New Mexico and Maui, Hawaii. Table 3.2 provides the geographic characteristics of the ground stations. The provided geodetic latitudes were transformed into geocentric latitudes for use in the simulation. Figure 3.1 shows the location of the ground stations and the starting location of the RSO projected onto a map of the Earth.

Table 3.2: GEODSS Ground Station Geographic Characteristics

\begin{tabular}{|c|c|c|}
\hline Geographic Characteristic & Socorro, NM & Maui, HI \\
\hline \hline Elevation (m) & 1510 & 3058 \\
\hline Geodetic Latitude (deg) & 33.817192 & 20.708400 \\
\hline Longitude (deg) & -106.659867 & -156.257433 \\
\hline
\end{tabular}




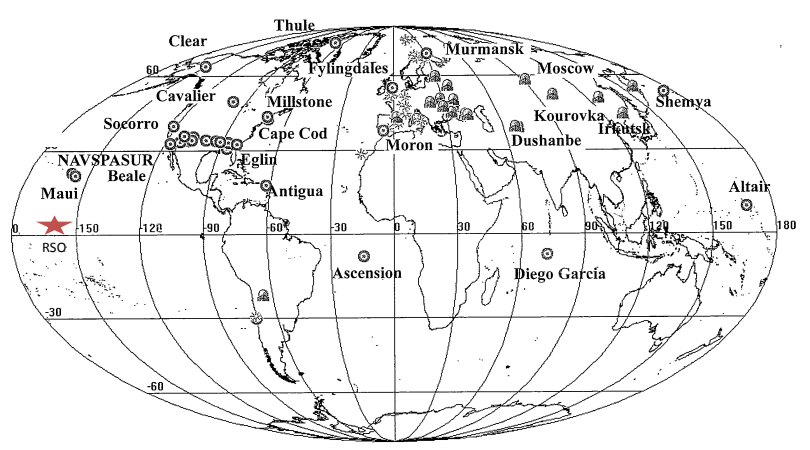

Fig. 3.1: Ground Station Locations and RSO Projected Starting Location (Image courtesy of Fundamentals of Astrodynamics and Applications, 4th Edition [1])

\subsubsection{Measurements}

Measurements of the RSO are performed by the ground stations and the space sensor throughout the simulation. Each space sensor observation consists of two measurements, right ascension and declination of the RSO. It is assumed that the space-based sensor performs in a sidereal tracking mode, which makes the stars appear as point sources and the RSOs appear as streaks across the field of view, similar to SBV [24]. The star background in the field of view is compared to a star catalog to determine the location the sensor is pointing, and then measurements of right ascension and declination are taken from the streaks of the RSOs compared to the stars. Measurements are chosen to occur near the closest approach of the space sensor to the RSO within a 7 day simulation period. In order for a measurement to be taken, sun lighting conditions on the RSO-space sensor geometry must be within constraints. Using Vallado's RSO phase angle description [12], the phase angle is the angle from the RSO-space sensor vector to the RSO-Sun vector. An angle of zero means the RSO is fully lit, 90 degrees is half lit, and 180 degrees is fully backlit. The maximum phase angle allowed in this analysis is 90 degrees. Nominally, measurements are performed every 25 seconds for a total of 5 minutes for a given measurement period. Measurements for each of the space sensor orbit scenarios were made to occur in the same timeframe of the 7 day simulation period. 
Each ground station observation consists of two measurements, right ascension and declination of the RSO. Nominally, measurements are performed every 25 seconds for a total of 6 minutes for a given measurement period. GEODSS uses a CCD combined with streak detection algorithms to gain observations of deep-space objects [25].

The right ascension, $\alpha$, and declination, $\delta$, measurement geometry is shown in Figure 3.2. For ground station measurements, the variable $\mathrm{O}$ marks the location of the ground station and the variable B marks the location of the RSO. For space sensor measurements, the variable $\mathrm{O}$ marks the location of the space sensor and the variable $\mathrm{B}$ marks the location of the RSO.

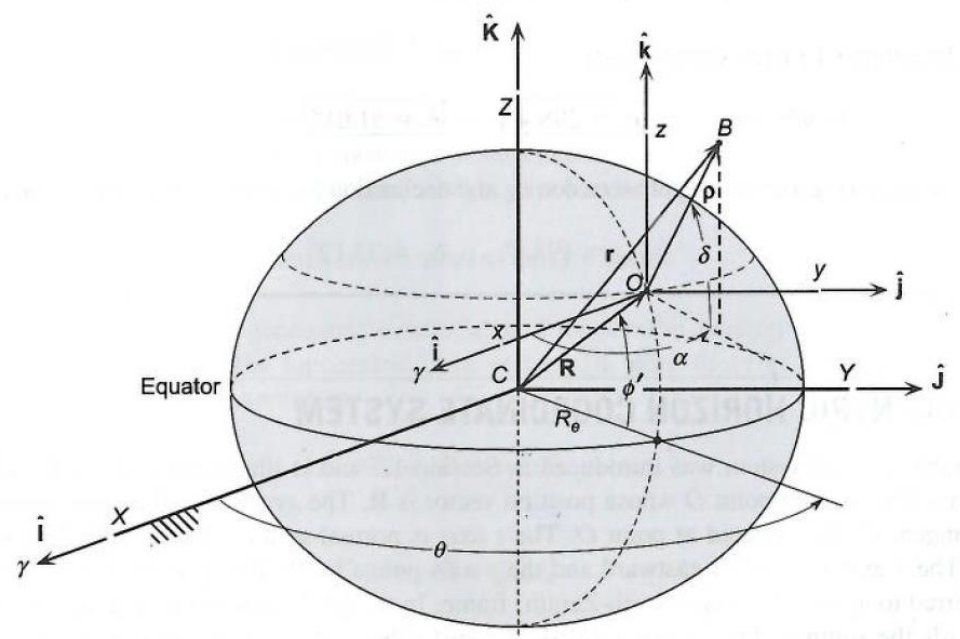

Fig. 3.2: Azimuth and Elevation Measurement Geometry (Image courtesy of Orbital Mechanics for Engineering Students, 2nd Edition [2])

Two space sensor navigation systems are simulated to update the space sensor position and velocity. The first option is GPS. GPS measurements are simulated as position and velocity updates on the space sensor. The baseline GPS measurement profile is a position and velocity measurement every 10 minutes. The second option is through the use of the SSC Universal Space Network ground stations. These measurements are simulated as a 
position and velocity measurement performed once per day.

All sensor measurements are modeled within this analysis to include an uncertain bias and noise. The bias and noise represent the imperfections that exist within the sensors, similar to SBV, to include: pixelization, distorted optics, and streak end point determination $[26]$.

\subsubsection{Tasking Profiles}

The starting time of all simulation analysis is set to zero in all plots; this zero time corresponds to February 10, 2009, 0000 hours.

Each ground station observation consists of two measurements, right ascension and declination of the RSO. Ground station measurements are set to occur using Vallado's GEODSS ground sensor tracking parameters for optical trackers as a guide [12]. For this analysis, the following is used:

Track Length: 6 minutes

Observation Step Size: 25 seconds

Revisit Time: 23 hours

Inter-revisit Time: 18 hours

Missed Track Probability: $35 \%$

Space sensor observations are set to occur near closet approach with the RSO. Measurement periods for all three space sensor orbits occur between the third and fourth ground station measurement periods. Figure 3.3 shows the nominal tasking profiles for each measurement source. The markers denote each measurement period start time. 


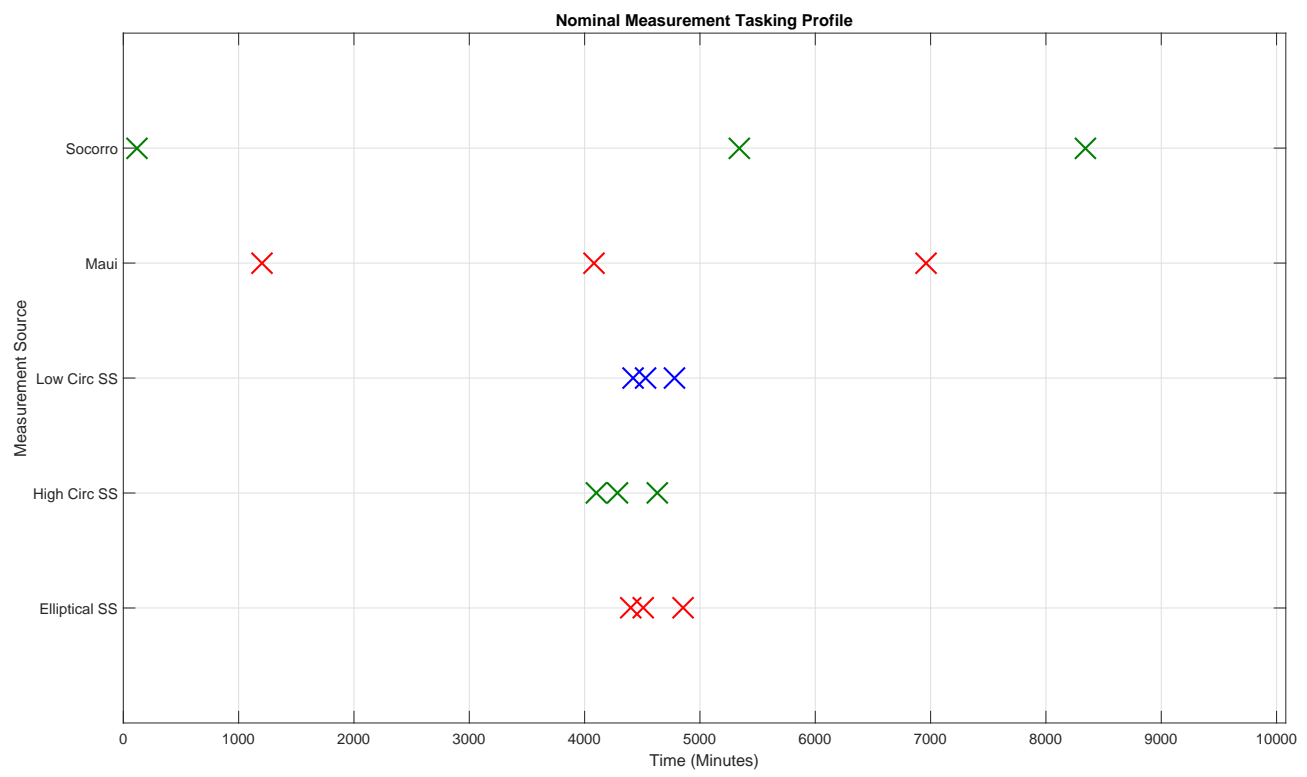

Fig. 3.3: Nominal Measurement Tasking Profiles

\subsection{Dynamics}

This section is a brief introduction to the dynamics included in this research effort, more detail is provided in Chapters 4 through 6 .

The RSO and space sensor are modeled to include the same dynamics. Consulting Montenbruck and Gill [27], the six most important perturbations at the geosynchronous orbit radius are:

1) Spherical Earth's gravity

2) $J_{2,0}$

3) Moon Third-Body

4) Sun Third-Body

5) Solar Radiation Pressure (SRP)

6) $J_{2,2}$

Each of these dominant perturbations are modeled for the RSO and the space sensor. In all developed analysis tools, the spherical harmonics gravity model programmed by 
Carlos Roithmayr, from NASA Langley Research Center, is used to model gravitational accelerations up to $J_{2,2}$. The code is based on NASA documents by Mueller and Roithmayr $[28,29]$.

The Moon and Sun third-body effects are modeled using the following equation [1]:

$$
\boldsymbol{a}_{\oplus s a t}=\mu_{3}\left[\frac{\boldsymbol{r}_{s a t 3}}{r_{s a t 3}^{3}}-\frac{\boldsymbol{r}_{\oplus 3}}{r_{\oplus 3}^{3}}\right]
$$

where:

$\mu_{3}$ : gravitational parameter of the third body

$\boldsymbol{r}_{\text {sat } 3}$ : vector from the satellite to the third body

$\boldsymbol{r}_{\oplus 3}$ : vector from Earth to the third body

The solar radiation pressure effect is modeled using a modified cannonball model for both the RSO and the space sensor. The cannonball model assumes the area that intersects the sunlight is always the same over time and the force direction is always away from the Sun. The equation used to model the acceleration due to SRP is [1]:

$$
\boldsymbol{a}_{S R P}=-p_{S R P}(C)(A) \frac{\boldsymbol{r}_{\text {sat } / \text { sun }}}{\left\|\boldsymbol{r}_{\text {sat } / \text { sun }}\right\|}
$$

where:

$p_{S R P}:$ solar radiation pressure

$C$ : coefficient of reflectivity

$A$ : area to mass ratio of the satellite facing the Sun

$\boldsymbol{r}_{\text {sat/sun }}$ : vector from satellite to the sun

The area to mass ratio of the RSO and the space sensor are modeled to change with time using a random walk.

In addition to the dominant perturbations, higher-order gravity terms above $J_{2,2}$ have an effect on the RSO and the space sensor and are modeled as exponentially random correlated variables. These will be referred to as disturbance accelerations. 
Finally, to account for accelerations that are not incorporated in the dynamics, such as the SRP dynamics of a high area to mass object or the effects from other planets, process noise is included on the geosynchronous object and space sensor. This will be referred to as random accelerations.

\subsection{Orbit Estimation Overview and Approach}

LinCov analysis and theory is used to generate the results of this dissertation. To develop the linear covariance model, the linear covariance analysis and error budget method provided by Maybeck [15] and Christensen and Geller [17] are utilized.

First, a truth model is developed that matches the real world as closely as possible. It encompasses the dynamics and measurements and includes the error sources described above. A Kalman filter is also developed, using the dynamics, measurements, and error sources described above, and processes the measurements generated by the truth model. The Kalman filter is then verified through use of Monte Carlo analysis. Once the Kalman filter is verified, it and the truth model are linearized around a reference trajectory to develop the linear covariance analysis tool. This linear covariance analysis tool is verified by comparison to the Monte Carlo results. Finally, the linear covariance analysis tool is used to generate the research results.

\subsection{Summary}

This chapter detailed the characteristics of the RSO and space sensor orbits, the ground station and space sensor tasking profiles, provided an overview of the dynamics incorporated, the measurements, and the orbit estimation/modeling approach taken. The next few chapters will detail the dynamics of the problem and present the estimation models created. 
CHAPTER 4

\section{MONTE CARLO SIMULATION-TRUTH MODELS}

\subsection{Nonlinear Truth Model}

The truth model is developed to represent, as closely as possible, the real world. It must incorporate all critical states to the estimation problem. The nonlinear truth model incorporates system dynamics models driven by white Gaussian noise and measurement models. The truth model generates measurements within the measurement models, which can have a bias and/or white Gaussian noise added to them.

The state vector, $\mathbf{x}$, represents the true state of the dynamic system. The dynamics of the truth states are a function of the truth states and noise

$$
\dot{\boldsymbol{x}}=f(\boldsymbol{x}, t)+\boldsymbol{w}
$$

where $\boldsymbol{w}$ is a vector of zero-mean white-noise processes that account for small uncertainties in the dynamics of the truth state, with covariance

$$
E\left[\boldsymbol{w}(t) \boldsymbol{w}^{T}\left(t^{\prime}\right)\right]=S_{w}(t) \delta\left(t-t^{\prime}\right)
$$

$E[\cdot]$ stands for the expected value of the quantity included in the brackets.

The discrete truth model measurements, $\tilde{\mathbf{z}}_{k}$, are a function of the truth states and noise

$$
\tilde{\mathbf{z}}_{k}=\boldsymbol{h}\left(\boldsymbol{x}_{k}, t_{k}\right)+\nu_{k}
$$

where $\nu_{k}$ is zero-mean discrete measurement noise, with covariance

$$
E\left[v_{k} v_{k^{\prime}}^{T}\right]=R_{\nu} \delta_{k k^{\prime}}
$$


For each analysis run, the truth model state vector is initialized with the nominal value of the state along with an additive random value defined by the initial 1-sigma dispersion of the initial state. All noise values are generated by a random number generator and defined by their initial 1-sigma value.

A detailed description of the true state dynamics and measurement models for the RSO estimation problem is given in Section 4.2.

\subsection{Truth Model Dynamics}

The orbital dynamics of the RSO and SS are given by

$$
\begin{gathered}
\dot{\mathbf{r}}_{r s o}=\mathbf{v}_{r s o} \\
\dot{\mathbf{v}}_{r s o}=\boldsymbol{a}_{18,18\left(\boldsymbol{r}_{r s o}\right)}+\boldsymbol{a}_{\text {moon }\left(\boldsymbol{r}_{r s o}\right)}+\boldsymbol{a}_{s u n\left(\boldsymbol{r}_{r s o}\right)}+\boldsymbol{a}_{S R P\left(\boldsymbol{r}_{r s o}\right)}+\boldsymbol{a}_{r s o}+\eta_{r s o} \\
\dot{\mathbf{r}}_{s s}=\mathbf{v}_{s s} \\
\dot{\mathbf{v}}_{s s}=\boldsymbol{a}_{18,18\left(\boldsymbol{r}_{s s}\right)}+\boldsymbol{a}_{m o o n\left(\boldsymbol{r}_{s s}\right)}+\boldsymbol{a}_{s u n\left(\boldsymbol{r}_{s s}\right)}+\boldsymbol{a}_{S R P\left(\boldsymbol{r}_{s s}\right)}+\boldsymbol{a}_{s s}+\eta_{s s}
\end{gathered}
$$

where $\boldsymbol{a}_{18,18}$ is a $18 \times 18$ spherical harmonics gravity model, $\boldsymbol{a}_{\text {moon/sun }}$ are third body perturbations as shown in Eq. 3.1, $\boldsymbol{a}_{S R P}$ is the acceleration due to SRP as shown in Eq. 3.2 with the area-to-mass ratio modeled as a random walk and the coefficient of reflectivity modeled as a constant

$$
\begin{gathered}
\dot{A}_{r s o}=\eta_{A_{r s o}}, \dot{C}_{r s o}=0 \\
\dot{A}_{s s}=\eta_{A_{s s}}, \dot{C}_{s s}=0 \\
E\left[\eta_{A_{r s o}}(t)\right]=E\left[\eta_{A_{S S}}(t)\right]=0
\end{gathered}
$$

with variance:

$$
E\left[\eta_{A_{r s o}}(t) \eta_{A_{r s o}}\left(t^{\prime}\right)\right]=Q_{A_{r s o}} \delta\left(t-t^{\prime}\right), E\left[\eta_{A_{S S}}(t) \eta_{A_{S S}}\left(t^{\prime}\right)\right]=Q_{A_{s s}} \delta\left(t-t^{\prime}\right)
$$


where $\eta_{A_{r s o}}$ and $\eta_{A_{S S}}$ are continuous Gaussian white noise processes, and $Q_{A_{r s o}}$ and $Q_{A_{s s}}$ is the strength of the white noise. $\boldsymbol{a}_{r s o}$ and $\boldsymbol{a}_{s s}$ are disturbance accelerations modeled as firstorder Markov processes, and $\eta_{r s o}$ and $\eta_{s s}$ are random accelerations modeled as continuous zero mean white noise

$$
\begin{gathered}
E\left[\eta_{r s o}(t)\right]=E\left[\eta_{s s}(t)\right]=0 \\
E\left[\eta_{r s o}(t) \eta_{r s o}\left(t^{\prime}\right)^{T}\right]=Q_{r s o} \delta\left(t-t^{\prime}\right), E\left[\eta_{s s}(t) \eta_{s s}\left(t^{\prime}\right)^{T}\right]=Q_{s s} \delta\left(t-t^{\prime}\right)
\end{gathered}
$$

where $Q_{r s o}$ and $Q_{s s}$ are the strength of the white noise.

\subsubsection{Spherical Harmonic Gravity Model}

Earth is not a perfectly spherical body, most of the mass that accounts for this is found near the equator, causing Earth to have an oblate shape. One approach of modeling the effect of mass distribution on Earth's gravity potential, which in turn affects the acceleration of a satellite, is modeling the gravity field in terms of a spherical harmonic series. The satellite acceleration due to Earth's gravity, in an Earth fixed frame, using spherical harmonics, is given by

$$
\begin{gathered}
\boldsymbol{r}^{E F}=T_{E F \rightarrow I}^{T} \boldsymbol{r} \\
\boldsymbol{a}_{18,18}^{E F}=\frac{\mu}{r}\left[\sum_{l=0}^{\infty} \sum_{m=0}^{l}\left(\frac{R_{\oplus}}{r}\right)^{l} P_{l, m}\left[\sin \left(\phi_{g c_{s a t}}\right)\right]\left\{C_{l, m} \cos \left(m \lambda_{s a t}\right)+S_{l, m} \sin \left(m \lambda_{s a t}\right)\right\}\right]
\end{gathered}
$$

where $l$ is the degree and $m$ is the order, for this analysis the degree and order are set to $18[1,28,29]$. To obtain this acceleration in the inertial frame in order to incorporate it into the dynamics equation, the Earth fixed acceleration is transformed back into the inertial frame.

$$
\boldsymbol{a}_{18,18}(\boldsymbol{r})=T_{E F \rightarrow I} \boldsymbol{a}_{18,18}^{E F}
$$




\subsubsection{Disturbance Accelerations}

The disturbance accelerations are modeled as first-order Markov processes, also known as an exponentially correlated random variable (ECRV):

$$
\dot{\boldsymbol{a}}_{r s o}=-\left(\frac{\mathbf{a}_{r s o}}{\tau_{r s o}}\right)+\eta_{\text {dist }_{r s o}}, \dot{\boldsymbol{a}}_{s s}=-\left(\frac{\mathbf{a}_{s s}}{\tau_{s s}}\right)+\eta_{\text {dist }_{s s}}
$$

where

$$
\begin{gathered}
E\left[\eta_{\text {dist }_{r s o}}(t)\right]=E\left[\eta_{\text {dist }_{s s}}(t)\right]=0 \\
E\left[\eta_{\text {dist }_{r s o}}(t) \eta_{\text {dist }_{r s o}}\left(t^{\prime}\right)^{T}\right]=\frac{2 \sigma_{r s o}^{2}}{\tau_{r s o}} \delta\left(t-t^{\prime}\right) \\
E\left[\eta_{\text {dist }_{s s}}(t) \eta_{\text {dist }_{s s}}\left(t^{\prime}\right)^{T}\right]=\frac{2 \sigma_{s s}^{2}}{\tau_{s s}} \delta\left(t-t^{\prime}\right)
\end{gathered}
$$

where $\eta_{\text {dist } t_{s o}}$ and $\eta_{\text {dist }}$ are continuous Gaussian white noise process, $\sigma_{r s o}^{2}$ and $\sigma_{s s}^{2}$ are the steady-state variance of the disturbance accelerations, and $\tau_{r s o}$ and $\tau_{s s}$ are time constants.

\subsubsection{Truth Model for Angle Only Measurements}

The angle only measurement models, which consist of the right ascension, $\alpha$, and declination, $\delta$, for each measurement source are provided in Eq. 4.22 through Eq. 4.27. These measurements include unknown measurement biases and noise.

$$
\begin{gathered}
\alpha_{G S 1}=\arctan \left(\frac{\rho_{1, j}}{\rho_{1, i}}\right)+b_{\alpha 1}+\eta_{\alpha 1} \\
\delta_{G S 1}=\arcsin \left(\frac{\rho_{1, k}}{\rho_{1}}\right)+b_{\delta 1}+\eta_{\delta 1} \\
\alpha_{G S 2}=\arctan \left(\frac{\rho_{2, j}}{\rho_{2, i}}\right)+b_{\alpha 2}+\eta_{\alpha 2} \\
\delta_{G S 2}=\arcsin \left(\frac{\rho_{2, k}}{\rho_{2}}\right)+b_{\delta 2}+\eta_{\delta 2} \\
\alpha_{s s}=\arctan \left(\frac{\rho_{s s, j}}{\rho_{s s, i}}\right)+b_{\alpha, s s}+\eta_{\alpha, s s}
\end{gathered}
$$




$$
\delta_{s s}=\arcsin \left(\frac{\rho_{s s, k}}{\rho_{s s}}\right)+b_{\delta, s s}+\eta_{\delta, s s}
$$

where $\rho_{1} \equiv\left\|\mathbf{r}_{r s o}-\mathbf{r}_{G S 1}\right\|, \rho_{2} \equiv\left\|\mathbf{r}_{r s o}-\mathbf{r}_{G S 2}\right\|, \rho_{s s} \equiv\left\|\mathbf{r}_{r s o}-\mathbf{r}_{s s}\right\|$ (all in inertial frame) and $\rho_{1, i / j / k}, \rho_{2, i / j / k}, \rho_{s s, i / j / k}$ are the inertial components.

The measurement biases are assumed to be constants [1]

$$
\begin{gathered}
\dot{b}_{\alpha 1}=0, \dot{b}_{\delta 1}=0 \\
\dot{b}_{\alpha 2}=0, \dot{b}_{\delta 2}=0 \\
\dot{b}_{\alpha, s s}=0, \dot{b}_{\delta, s s}=0
\end{gathered}
$$

All noise is modeled as zero-mean white Gaussian noise processes with variances:

$$
\begin{gathered}
E\left[\eta_{\alpha 1}^{2}(t)\right]=\sigma_{\eta_{\alpha 1}}^{2}, E\left[\eta_{\delta 1}^{2}(t)\right]=\sigma_{\eta_{\delta 1}}^{2} \\
E\left[\eta_{\alpha 2}^{2}(t)\right]=\sigma_{\eta_{\alpha 2}}^{2}, E\left[\eta_{\delta 2}^{2}(t)\right]=\sigma_{\eta_{\delta 2}}^{2} \\
E\left[\eta_{\alpha, s s}^{2}(t)\right]=\sigma_{\eta_{\alpha, s s}}^{2}, E\left[\eta_{\delta, s s}^{2}(t)\right]=\sigma_{\eta_{\delta, s s}}^{2}
\end{gathered}
$$

\subsubsection{Truth Model for GPS/USN Measurements}

The space sensor position and velocity is updated at regular intervals through the simulation by use of GPS updates or USN ground tracking station updates. This measurement is generated as the current position and velocity of the space sensor with an additive noise. The noise is modeled as zero-mean white Gaussian noise processes with variances:

$$
\begin{gathered}
G P S_{r}=\boldsymbol{r}_{s s}+\eta_{g p s, r} \\
G P S_{v}=\boldsymbol{v}_{s s}+\eta_{g p s, v} \\
E\left[\eta_{g p s, r}^{2}(t)\right]=\sigma_{g p s, r}^{2}, E\left[\eta_{g p s, v}^{2}(t)\right]=\sigma_{g p s, v}^{2}
\end{gathered}
$$




\subsection{Truth State Vector}

The truth state for the Monte Carlo analysis is a 28-dimensional vector, as given by

$$
\boldsymbol{x}=\left[\begin{array}{c}
\boldsymbol{x}_{r s o} \\
\boldsymbol{x}_{s s} \\
\boldsymbol{p}_{\text {sensor }} \\
\boldsymbol{p}_{\text {dist }}
\end{array}\right]
$$

where

$$
\boldsymbol{x}_{r s o}=\left[\begin{array}{c}
\boldsymbol{r}_{r s o} \\
\boldsymbol{v}_{r s o} \\
C_{r s o} \\
A_{r s o}
\end{array}\right]
$$

where $\boldsymbol{r}_{r s o}$ is the inertial position of the GEO RSO, $\boldsymbol{v}_{r s o}$ is the inertial velocity of the GEO $\mathrm{RSO}, C_{r s o}$ is the RSO coefficient of reflectivity, and $A_{r s o}$ is the RSO area-to-mass ratio.

$$
\boldsymbol{x}_{s s}=\left[\begin{array}{c}
\boldsymbol{r}_{s s} \\
\boldsymbol{v}_{s s} \\
C_{s s} \\
A_{s s}
\end{array}\right]
$$

where $\boldsymbol{r}_{s s}$ is the inertial position of the space sensor, $\boldsymbol{v}_{s s}$ is the inertial velocity of the space sensor, $C_{s s}$ is the space sensor coefficient of reflectivity, and $A_{s s}$ is the space sensor 
area-to-mass ratio.

$$
\boldsymbol{p}_{\text {sensor }}=\left[\begin{array}{c}
b_{\alpha 1} \\
b_{\delta 1} \\
b_{\alpha 2} \\
b_{\delta 2} \\
b_{\alpha, s s} \\
b_{\delta, s s}
\end{array}\right]
$$

which consists of the right ascension and declination measurement biases for each measurement source.

$$
\boldsymbol{p}_{\text {dist }}=\left[\begin{array}{c}
\boldsymbol{a}_{r s o} \\
\boldsymbol{a}_{s s}
\end{array}\right]
$$

where $\boldsymbol{a}_{r s o}$ is the disturbance accelerations on the RSO and $\boldsymbol{a}_{s s}$ is the disturbance accelerations on the space sensor.

\subsection{Summary}

This chapter provided all of the equations used to build the truth model for the RSO estimation problem. The developed truth model is used to perform Monte Carlo analysis and is a fundamental piece of creating the LinCov analysis tool. 
CHAPTER 5

\section{MONTE CARLO SIMULATION-EXTENDED KALMAN FILTER}

\subsection{Extended Kalman Filter Overview}

The extended Kalman filter produces a state estimate. The vector, $\hat{\boldsymbol{x}}$, represents the estimate of the state. The estimated state is propagated and updated using Eq. 5.1 through Eq. 5.5 below.

$$
\begin{gathered}
\dot{\hat{\boldsymbol{x}}}=\hat{f}(\hat{\boldsymbol{x}}, t) \\
\hat{\boldsymbol{x}}_{k}^{+}=\hat{\boldsymbol{x}}_{k}^{-}+\hat{K}_{k}\left[\tilde{\mathbf{z}}_{k}-\hat{\tilde{\mathbf{z}}}_{k}\right]
\end{gathered}
$$

$\hat{\tilde{\mathbf{z}}}_{k}$ is the estimated value of the measurement:

$$
\hat{\tilde{\mathbf{z}}}_{k}=\hat{\boldsymbol{h}}\left(\hat{\boldsymbol{x}}_{k}, t_{k}\right)
$$

The Kalman gain, $\hat{K}_{k}$, is determined using:

$$
\hat{K}_{k}=\hat{P}_{k}^{-} \hat{H}_{k}^{T}\left(\hat{H}_{k} \hat{P}_{k}^{-} \hat{H}_{k}^{T}+\hat{R}_{v}\right)^{-1}
$$

where:

$$
\hat{H}_{k}=\left.\frac{\partial \hat{\boldsymbol{h}}\left(\hat{\boldsymbol{x}}_{k}, t_{k}\right)}{\partial \hat{\boldsymbol{x}}_{k}}\right|_{\hat{\boldsymbol{x}}}
$$

The filter covariance, $\hat{P}_{k}$, is propagated according to Eq. 5.6.

$$
\hat{P}_{i+1}^{+}=\Phi_{i} \hat{P}_{i} \Phi_{i}^{T}+\hat{Q}_{d, i}
$$

where $\Phi$, the state transition matrix to the second order, is equal to:

$$
\Phi=e^{\hat{F}\left(t-t_{0}\right)}=I_{n x n}+\hat{F}\left(t-t_{0}\right)+\hat{F}^{2}\left(t-t_{0}\right)^{2} / 2 !
$$


and where the Jacobian is given by:

$$
\hat{F}=\left.\frac{\partial \hat{f}(\hat{\boldsymbol{x}}, t)}{\partial \hat{\boldsymbol{x}}}\right|_{\hat{\boldsymbol{x}}}
$$

The filter covariance, $\hat{P}_{k}$, is updated according to Eq. 5.9.

$$
\hat{P}_{k}^{+}=\left(I-\hat{K}_{k} \hat{H}_{k}\right) \hat{P}_{k}^{-}\left(I-\hat{K}_{k} \hat{H}_{k}\right)^{T}+\hat{K}_{k} \hat{R}_{v} \hat{K}_{k}^{T}
$$

The variables $\hat{F}, \hat{H}, \hat{Q}_{d, i}$, and $\hat{R}_{v}$ are determined by the filter design model. A detailed description of the EKF dynamics and measurement models for the RSO estimation problem are given in Section 5.2.

\subsection{EKF Model Dynamics}

The orbital dynamics of the RSO and SS are given by

$$
\begin{gathered}
\dot{\hat{\boldsymbol{r}}}_{r s o}=\hat{\boldsymbol{v}}_{r s o} \\
\dot{\hat{\boldsymbol{v}}}_{r s o}=\boldsymbol{a}_{2,2\left(\hat{\boldsymbol{r}}_{r s o}\right)}+\boldsymbol{a}_{m o o n\left(\hat{\boldsymbol{r}}_{r s o}\right)}+\boldsymbol{a}_{s u n\left(\hat{\boldsymbol{r}}_{r s o}\right)}+\boldsymbol{a}_{S R P\left(\hat{\boldsymbol{r}}_{r s o}\right)}+\boldsymbol{a}_{r s o} \\
\dot{\hat{\boldsymbol{r}}}_{s s}=\hat{\boldsymbol{v}}_{s s} \\
\dot{\hat{\boldsymbol{v}}}_{s s}=\boldsymbol{a}_{2,2\left(\hat{\boldsymbol{r}}_{s s}\right)}+\boldsymbol{a}_{m o o n\left(\hat{\boldsymbol{r}}_{s s}\right)}+\boldsymbol{a}_{\text {sun }\left(\hat{\boldsymbol{r}}_{s s}\right)}+\boldsymbol{a}_{S R P\left(\hat{\boldsymbol{r}}_{s s}\right)}+\boldsymbol{a}_{s s}
\end{gathered}
$$

where $\boldsymbol{a}_{2,2}$ is a $2 \times 2$ spherical harmonics gravity model, $\boldsymbol{a}_{\text {moon/sun }}$ are third body perturbations as shown in Eq. 3.1, $\boldsymbol{a}_{S R P}$ is the acceleration due to SRP as shown in Eq. 3.2 with the area-to-mass ratio is modeled as a random walk and the coefficient of reflectivity modeled as a constant.

$$
\begin{gathered}
\dot{\hat{\hat{A}}}_{r s o}=0, \dot{\hat{C}}_{r s o}=0 \\
\dot{\hat{A}}_{s s}=0, \dot{\hat{C}}_{s s}=0
\end{gathered}
$$

$\boldsymbol{a}_{r s o}$ and $\boldsymbol{a}_{s s}$ are disturbance accelerations modeled as first-order Markov processes. 


\subsubsection{Spherical Harmonic Gravity Model}

The EKF incorporates a spherical harmonic gravity model of degree and order 2. This is to represent the most influential gravitational forces acting on the RSO and SS. As mentioned previously, the higher-order gravity terms, referred to as disturbance accelerations, will be represented by an ECRV within the EKF.

The satellite acceleration due to Earth's gravity, in an Earth fixed frame, using spherical harmonics, is given by

$$
\begin{gathered}
\hat{\boldsymbol{r}}^{E F}=T_{E F \rightarrow I}^{T} \hat{\boldsymbol{r}} \\
\boldsymbol{a}_{2,2}^{E F}=\frac{\mu}{\hat{r}}\left[\sum_{l=0}^{\infty} \sum_{m=0}^{l}\left(\frac{R_{\oplus}}{\hat{r}}\right)^{l} P_{l, m}\left[\sin \left(\phi_{g c_{s a t}}\right)\right]\left\{C_{l, m} \cos \left(m \lambda_{\text {sat }}\right)+S_{l, m} \sin \left(m \lambda_{\text {sat }}\right)\right\}\right]
\end{gathered}
$$

where $l$ is the degree and $m$ is the order, for this analysis the degree and order are set to $2[1,28,29]$. To obtain this acceleration in the inertial frame in order to incorporate it into the dynamics equation, the Earth fixed acceleration is transformed back into the inertial frame.

$$
\boldsymbol{a}_{2,2}(\hat{\boldsymbol{r}})=T_{E F \rightarrow I} \boldsymbol{a}_{2,2}^{E F}
$$

\subsubsection{Disturbance Accelerations}

The disturbance accelerations are modeled as first-order Markov processes, also known as an exponentially correlated random variable (ECRV):

$$
\dot{\hat{\boldsymbol{a}}}_{r s o}=-\left(\frac{\hat{\mathbf{a}}_{r s o}}{\tau_{r s o}}\right), \dot{\hat{\boldsymbol{a}}}_{s s}=-\left(\frac{\hat{\mathbf{a}}_{s s}}{\tau_{s s}}\right)
$$

where $\tau_{r s o}$ and $\tau_{s s}$ are time constants.

\subsubsection{EKF Angles-Only Measurement Model}

The estimated angle only measurement models which consist of the right ascension, $\alpha$, and declination, $\delta$, for each measurement source are provided in Eq. 5.20 through Eq. 5.25. 


$$
\begin{aligned}
& \hat{\tilde{\alpha}}_{G S 1}=\arctan \left(\frac{\hat{\rho}_{1, j}}{\hat{\rho}_{1, i}}\right)+\hat{b}_{\alpha 1} \\
& \hat{\tilde{\delta}}_{G S 1}=\arcsin \left(\frac{\hat{\rho}_{1, k}}{\hat{\rho}_{1}}\right)+\hat{b}_{\delta 1} \\
& \hat{\tilde{\alpha}}_{G S 2}=\arctan \left(\frac{\hat{\rho}_{2, j}}{\hat{\rho}_{2, i}}\right)+\hat{b}_{\alpha 2} \\
& \hat{\tilde{\delta}}_{G S 2}=\arcsin \left(\frac{\hat{\rho}_{2, k}}{\hat{\rho}_{2}}\right)+\hat{b}_{\delta 2} \\
& \hat{\tilde{\alpha}}_{s s}=\arctan \left(\frac{\hat{\rho}_{s s, j}}{\hat{\rho}_{s s, i}}\right)+\hat{b}_{\alpha, s s} \\
& \hat{\tilde{\delta}}_{s s}=\arcsin \left(\frac{\hat{\rho}_{s s, k}}{\hat{\rho}_{s s}}\right)+\hat{b}_{\delta, s s}
\end{aligned}
$$

where $\hat{\rho}_{1} \equiv\left\|\hat{\mathbf{r}}_{r s o}-\hat{\mathbf{r}}_{G S 1}\right\|, \hat{\rho}_{2} \equiv\left\|\hat{\mathbf{r}}_{r s o}-\hat{\mathbf{r}}_{G S 2}\right\|, \hat{\rho}_{s s} \equiv\left\|\hat{\mathbf{r}}_{r s o}-\hat{\mathbf{r}}_{s s}\right\|$ (all in inertial frame) and $\hat{\rho}_{1, i / j / k}, \hat{\rho}_{2, i / j / k}, \hat{\rho}_{s s, i / j / k}$ are the inertial components.

The measurement biases are modeled as constants [1].

$$
\begin{gathered}
\dot{\hat{b}}_{\alpha 1}=0, \dot{\hat{b}}_{\delta 1}=0 \\
\dot{\hat{b}}_{\alpha 2}=0, \dot{\hat{b}}_{\delta 2}=0 \\
\dot{\hat{b}}_{\alpha, s s}=0, \dot{\hat{b}}_{\delta, s s}=0
\end{gathered}
$$

\subsubsection{EKF GPS/USN Measurement Model}

The estimated GPS/USN measurement update is modeled as equivalent to the most recent estimate of the space sensor position and velocity.

$$
\begin{aligned}
G \hat{\tilde{P}} S_{r} & =\hat{\boldsymbol{r}}_{s s} \\
G \hat{\tilde{P}} S_{v} & =\hat{\boldsymbol{v}}_{s s}
\end{aligned}
$$




\subsection{EKF State Vector}

The EKF state for the Monte Carlo analysis is a 28-dimensional vector, as given by

$$
\hat{\boldsymbol{x}}=\left[\begin{array}{c}
\hat{\boldsymbol{x}}_{r s o} \\
\hat{\boldsymbol{x}}_{s s} \\
\hat{\boldsymbol{p}}_{\text {sensor }} \\
\hat{\boldsymbol{p}}_{\text {dist }}
\end{array}\right]
$$

where

$$
\hat{\boldsymbol{x}}_{r s o}=\left[\begin{array}{c}
\hat{\boldsymbol{r}}_{r s o} \\
\hat{\boldsymbol{v}}_{r s o} \\
\hat{C}_{r s o} \\
\hat{A}_{r s o}
\end{array}\right]
$$

where $\hat{\boldsymbol{r}}_{r s o}$ is the estimated inertial position of the GEO RSO, $\hat{\boldsymbol{v}}_{r s o}$ is the estimated inertial velocity of the GEO RSO, $\hat{C}_{r s o}$ is the estimated RSO coefficient of reflectivity, and $\hat{A}_{r s o}$ is the estimated RSO area-to-mass ratio.

$$
\hat{\boldsymbol{x}}_{s s}=\left[\begin{array}{c}
\hat{\boldsymbol{r}}_{s s} \\
\hat{\boldsymbol{v}}_{s s} \\
\hat{C}_{s s} \\
\hat{A}_{s s}
\end{array}\right]
$$

where $\hat{\boldsymbol{r}}_{s s}$ is the estimated inertial position of the space sensor, $\hat{\boldsymbol{v}}_{s s}$ is the estimated inertial velocity of the space sensor, $\hat{C}_{s s}$ is the estimated space sensor coefficient of reflectivity, and 
$\hat{A}_{s s}$ is the estimated space sensor area-to-mass ratio.

$$
\hat{\boldsymbol{p}}_{\text {sensor }}=\left[\begin{array}{c}
\hat{b}_{\alpha 1} \\
\hat{b}_{\delta 1} \\
\hat{b}_{\alpha 2} \\
\hat{b}_{\delta 2} \\
\hat{b}_{\alpha, s s} \\
\hat{b}_{\delta, s s}
\end{array}\right]
$$

which consists of the estimated right ascension and declination measurement biases for each measurement source.

$$
\hat{\boldsymbol{p}}_{d i s t}=\left[\begin{array}{c}
\hat{\boldsymbol{a}}_{r s o} \\
\hat{\boldsymbol{a}}_{s s}
\end{array}\right]
$$

where $\hat{\boldsymbol{a}}_{r s o}$ is the disturbance accelerations on the RSO and $\hat{\boldsymbol{a}}_{s s}$ is the disturbance accelerations on the space sensor.

\subsection{Covariance Propagation and the Jacobian}

In order to propagate the EKF state covariance as shown in Eq.5.6, the partial deriva-

tive matrix $\hat{F}$ and $\hat{Q}_{d, i}$ must be determined. For this analysis, the Jacobian, $\hat{F}$, matrix becomes: 


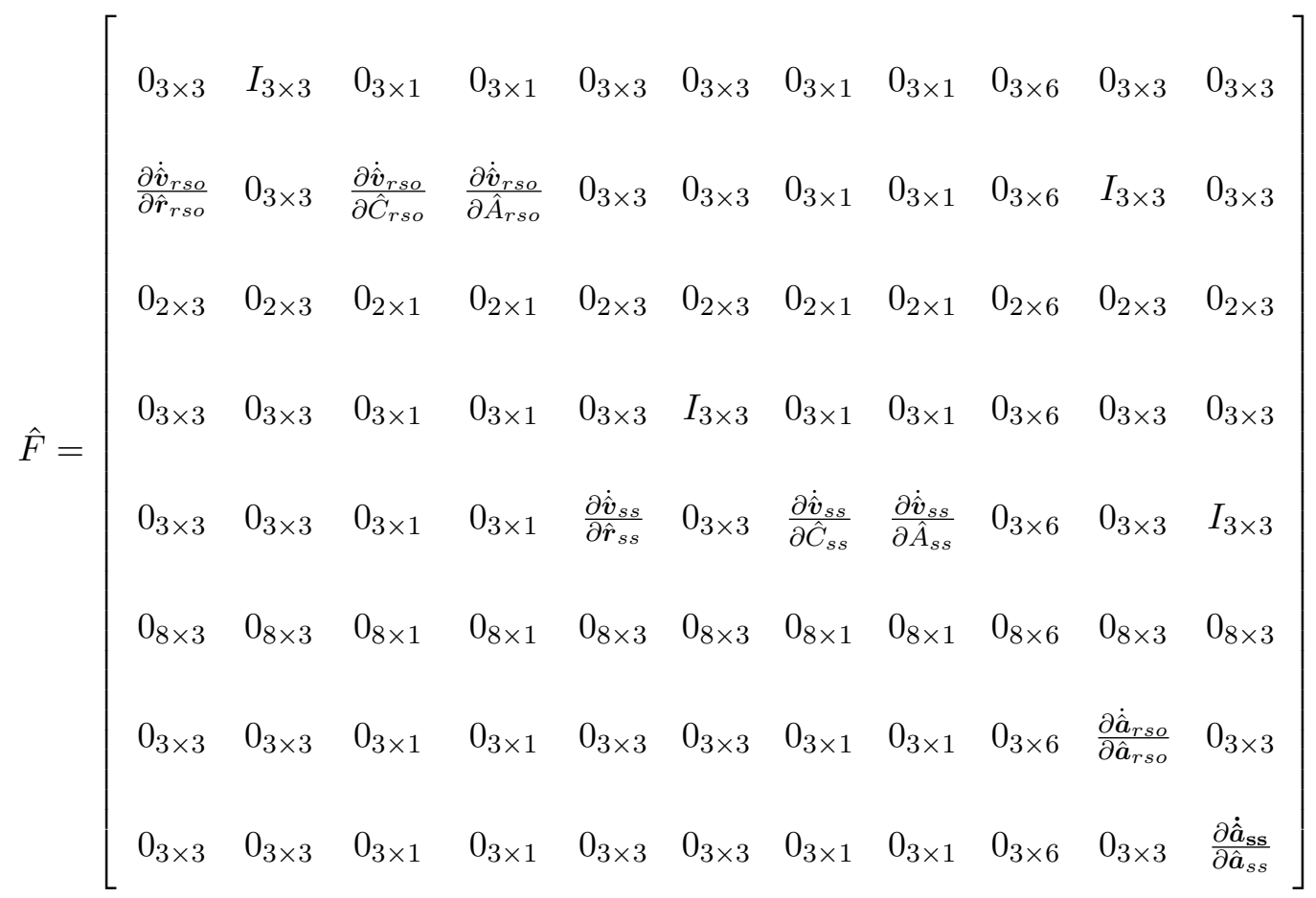

where:

$$
\begin{gathered}
\frac{\partial \dot{\hat{\boldsymbol{v}}}}{\partial \hat{C}}=-P_{S R P}(\hat{A})\left(\frac{\rho_{\text {Sun }}-\hat{\boldsymbol{r}}}{\left\|\rho_{\text {Sun }}-\hat{\boldsymbol{r}}\right\|}\right) \\
\frac{\partial \dot{\hat{\boldsymbol{v}}}}{\partial \hat{A}}=-P_{\text {SRP }}(\hat{C})\left(\frac{\rho_{\text {Sun }}-\hat{\boldsymbol{r}}}{\left\|\rho_{\text {Sun }}-\hat{\boldsymbol{r}}\right\|}\right) \\
\frac{\partial \dot{\hat{\boldsymbol{a}}}}{\partial \hat{\boldsymbol{a}}}=\frac{-1}{\tau} I_{3 x 3}
\end{gathered}
$$

and

$$
\frac{\partial \dot{\hat{\boldsymbol{v}}}}{\partial \hat{\boldsymbol{r}}}=\frac{\partial \boldsymbol{a}_{2,2}}{\partial \hat{\boldsymbol{r}}}+\frac{\partial \boldsymbol{a}_{\text {moon }}}{\partial \hat{\boldsymbol{r}}}+\frac{\partial \boldsymbol{a}_{\text {sun }}}{\partial \hat{\boldsymbol{r}}}+\frac{\partial \boldsymbol{a}_{S R P}}{\partial \hat{\boldsymbol{r}}}
$$


where

$$
\begin{gathered}
\frac{\partial \boldsymbol{a}_{2,2}}{\partial \hat{\boldsymbol{r}}}=-\frac{\mu}{\hat{r}^{3}}\left(-3 \hat{i}^{T}+I_{3 x 3}\right)-\frac{\mu(J 2)\left(R_{\oplus}^{2}\right)}{2} \\
\left\{\left(\frac{-30\left(\boldsymbol{n}^{T} \hat{\boldsymbol{r}}\right) \boldsymbol{n}\left(\hat{i}^{T}\right)}{\hat{r}^{6}}\right)+\left(\frac{6\left(\boldsymbol{n} \boldsymbol{n}^{T}\right)}{\hat{r}^{5}}\right)-\left(\frac{15 \hat{\boldsymbol{r}}\left(\hat{i}^{T}\right)}{\hat{r}^{6}}\right)+\left(\frac{3\left(I_{3 x 3}\right)}{\hat{r}^{5}}\right)+\right. \\
\left.\left(\frac{75\left(\hat{i}^{T} \boldsymbol{n}\right)^{2} \hat{\mathbf{r}}\left(\hat{i}^{T}\right)}{\hat{r}^{6}}\right)-\left(\frac{15\left(\hat{i}^{T} \boldsymbol{n}\right) I_{3 x 3}}{\hat{r}^{5}}\right)-\left(\frac{30 \hat{\boldsymbol{r}}\left(\boldsymbol{n}^{T} \hat{i}\right) \boldsymbol{n}^{T}}{\hat{r}^{6}}\right)\left(I_{3 x 3}-\hat{i}^{T}\right)\right\} \\
\boldsymbol{n}=\left[\begin{array}{lll}
0 & 0 & 1
\end{array}\right]^{T}
\end{gathered}
$$

Note: This partial derivative includes only $J_{2,0}$ and does not include the partial derivative for $J_{2,2} . J_{2,2}$, referencing Montenbruck and Gill [27], is the smallest of the six most important and included perturbations at GEO. To see the effect of neglecting the $J_{2,2}$ partial derivative, analysis was done which compared results of a filter with and without a $J_{2,2}$ numerical partial derivative, using an $18 \times 18$ gravity model for the truth. The resulting difference, when small initial conditions (given in Table 6.1) were used on the RSO, was an ending RMS 3-sigma error of only 30.1 meters, where the overall 3-sigma error was 111.7 $\mathrm{km}$. Thus, although terms up to $J_{2,2}$ are included in the filter gravity model, the Jacobian includes only terms up to $J_{2,0}$.

$$
\begin{gathered}
\frac{\partial \boldsymbol{a}_{\text {moon }}}{\partial \hat{\boldsymbol{r}}}=\left(3 \mu_{\text {Moon }} \frac{\hat{\boldsymbol{r}}-\rho_{\text {Moon }}}{\left\|\hat{\boldsymbol{r}}-\rho_{\text {Moon }}\right\|^{4}} \frac{\left(\hat{\boldsymbol{r}}-\rho_{\text {Moon }}\right)^{T}}{\left\|\hat{\boldsymbol{r}}-\rho_{\text {Moon }}\right\|}\right)-\frac{\mu_{\text {Moon }}}{\left\|\hat{\boldsymbol{r}}-\rho_{\text {Moon }}\right\|^{3}} I_{3 x 3} \\
\frac{\partial \boldsymbol{a}_{\text {sun }}}{\partial \hat{\boldsymbol{r}}}=\left(3 \mu_{\text {Sun }} \frac{\hat{\boldsymbol{r}}-\rho_{\text {Sun }}}{\left\|\hat{\boldsymbol{r}}-\rho_{\text {Sun }}\right\|^{4}} \frac{\left(\hat{\boldsymbol{r}}-\rho_{\text {Sun }}\right)^{T}}{\left\|\hat{\boldsymbol{r}}-\rho_{\text {Sun }}\right\|}\right)-\frac{\mu_{\text {Sun }}}{\left\|\hat{\boldsymbol{r}}-\rho_{\text {Sun }}\right\|^{3}} I_{3 x 3} \\
\frac{\partial \boldsymbol{a}_{\text {SRP }}}{\partial \hat{\boldsymbol{r}}}=-P_{S R P}(\hat{C})(\hat{A})\left(\frac{-I_{3 x 3}}{\left\|\rho_{\text {Sun }}-\hat{\boldsymbol{r}}\right\|}+\frac{\rho_{\text {Sun }}-\hat{\boldsymbol{r}}}{\left\|\rho_{\text {Sun }}-\hat{\boldsymbol{r}}\right\|^{2}} \frac{\left(\rho_{\text {Sun }}-\hat{\boldsymbol{r}}\right)^{T}}{\left\|\rho_{\text {Sun }}-\hat{\boldsymbol{r}}\right\|} I_{3 x 3}\right) \\
\frac{\partial \dot{\hat{\boldsymbol{v}}}}{\partial \hat{C}}=-P_{S R P}(\hat{A})\left(\frac{\rho_{\text {Sun }}-\hat{\boldsymbol{r}}}{\left\|\rho_{\text {Sun }}-\hat{\boldsymbol{r}}\right\|}\right) \\
\frac{\partial \dot{\hat{\boldsymbol{v}}}}{\partial \hat{A}}=-P_{S R P}(\hat{C})\left(\frac{\rho_{\text {Sun }}-\hat{\boldsymbol{r}}}{\left\|\rho_{\text {Sun }}-\hat{\boldsymbol{r}}\right\|}\right)
\end{gathered}
$$


The $\hat{Q}_{d, i}$ matrix is:

$$
\hat{Q}_{d, i}=\left[\begin{array}{cc}
\hat{Q}_{d 1, i} & 0_{14 x 14} \\
0_{14 x 14} & \hat{Q}_{d 2, i}
\end{array}\right]
$$

where:

$$
\begin{aligned}
& \hat{Q}_{d 1, i}=\left[\begin{array}{cccccc}
0_{3 \times 3} & 0_{3 \times 3} & 0_{3 \times 1} & 0_{3 \times 1} & 0_{3 \times 3} & 0_{3 \times 3} \\
0_{3 \times 3} & \hat{Q}_{r s o} \Delta t & 0_{3 \times 1} & 0_{3 \times 1} & 0_{3 \times 3} & 0_{3 \times 3} \\
0_{1 \times 3} & 0_{1 \times 3} & 0_{1 \times 1} & 0_{1 \times 1} & 0_{1 \times 3} & 0_{1 \times 3} \\
0_{1 \times 3} & 0_{1 \times 3} & 0_{1 \times 1} & \hat{Q}_{A_{r s o}} \Delta t & 0_{1 \times 3} & 0_{1 \times 3} \\
0_{3 \times 3} & 0_{3 \times 3} & 0_{3 \times 1} & 0_{3 \times 1} & 0_{3 \times 3} & 0_{3 \times 3} \\
0_{3 \times 3} & 0_{3 \times 3} & 0_{3 \times 1} & 0_{3 \times 1} & 0_{3 \times 3} & \hat{Q}_{s s} \Delta t
\end{array}\right] \\
& \hat{Q}_{d 2, i}=\left[\begin{array}{ccccc}
0_{1 \times 1} & 0_{1 \times 1} & 0_{1 \times 6} & 0_{1 \times 3} & 0_{1 \times 3} \\
0_{1 \times 1} & \hat{Q}_{A_{s s}} \Delta t & 0_{1 \times 6} & 0_{1 \times 3} & 0_{1 \times 3} \\
0_{6 \times 1} & 0_{6 \times 1} & 0_{6 \times 6} & 0_{6 \times 3} & 0_{6 \times 3} \\
0_{3 \times 1} & 0_{3 \times 1} & 0_{3 \times 6} & \frac{2 \hat{\sigma}_{r s o}^{2}}{\tau_{r s o}} \triangle t & 0_{3 \times 3} \\
0_{3 \times 1} & 0_{3 \times 1} & 0_{3 \times 6} & 0_{3 \times 3} & \frac{2 \hat{\sigma}_{s s}^{2}}{\tau_{s s}} \triangle t
\end{array}\right]
\end{aligned}
$$

\subsection{EKF Covariance Update}

In order to update the state and covariance in the EKF, the partial derivative matrix $\hat{H}_{k}$ and $\hat{R}_{v}$ must be determined. The $\hat{H}_{k}$ matrix consists of four separate matrices, each supporting one of the measurement sources. When a particular measurement source is used to obtain a measurement, the $\hat{H}_{k}$ matrix corresponding to that source is used. 


$$
\begin{aligned}
\hat{H}_{k, 1}= & {\left[\begin{array}{cccccc}
\frac{\partial \alpha_{G S 1}}{\partial \hat{\boldsymbol{r}}_{r s o}} & 0_{1 \times 5} & 0_{1 \times 3} & 0_{1 \times 5} & \frac{\partial \alpha_{G S 1}}{\partial \hat{b}_{\alpha 1}} & 0_{1 \times 11} \\
\frac{\partial \delta_{G S 1}}{\partial \hat{r}_{r s o}} & 0_{1 \times 5} & 0_{1 \times 3} & 0_{1 \times 6} & \frac{\partial \delta_{G S 1}}{\partial \hat{b}_{\delta 1}} & 0_{1 \times 10}
\end{array}\right] } \\
\hat{H}_{k, 2}= & {\left[\begin{array}{cccccc}
\frac{\partial \alpha_{G S 2}}{\partial \hat{\boldsymbol{r}}_{r s o}} & 0_{1 \times 5} & 0_{1 \times 3} & 0_{1 \times 7} & \frac{\partial \alpha_{G S 2}}{\partial \hat{b}_{\alpha 2}} & 0_{1 \times 9} \\
\frac{\partial \delta_{G S 2}}{\partial \hat{\boldsymbol{r}}_{r s o}} & 0_{1 \times 5} & 0_{1 \times 3} & 0_{1 \times 8} & \frac{\partial \delta_{G S 2}}{\partial_{\hat{b}_{\delta 2}}} & 0_{1 \times 8}
\end{array}\right] } \\
\hat{H}_{k, s s}= & {\left[\begin{array}{cccccc}
\frac{\partial \alpha_{S S}}{\partial \hat{\boldsymbol{r}}_{r s o}} & 0_{1 \times 5} & \frac{\partial \alpha_{S S}}{\partial \hat{\boldsymbol{r}}_{s s}} & 0_{1 \times 9} & \frac{\partial \alpha_{S S}}{\partial \hat{b}_{\alpha, s s}} & 0_{1 \times 7} \\
\frac{\partial \delta_{S S}}{\partial \hat{\boldsymbol{r}}_{r s o}} & 0_{1 \times 5} & \frac{\partial \delta_{S S}}{\partial \hat{\boldsymbol{r}}_{s s}} & 0_{1 \times 10} & \frac{\partial \delta_{S S}}{\partial \hat{b}_{\delta s s}} & 0_{1 \times 6}
\end{array}\right] } \\
\hat{H}_{k, g p s}= & {\left[\begin{array}{lllll} 
\\
0_{3 \times 8} & I_{3 \times 3} & 0_{3 \times 3} & 0_{3 \times 14} & \\
0 & & & & \\
03 \times 8 & 0_{3 \times 3} & I_{3 \times 3} & 0_{3 \times 14}
\end{array}\right] }
\end{aligned}
$$

where:

$$
\begin{aligned}
& \frac{\partial \alpha_{G S 1}}{\partial \hat{\boldsymbol{r}}_{r s o}}=\left[\begin{array}{lll}
-\frac{\hat{\rho}_{1, j}}{\left(\hat{\rho}_{1, i}+\hat{\rho}_{1, j}^{2}\right)} & \frac{\hat{\rho}_{1, i}}{\left(\hat{\rho}_{1, i}^{2}+\hat{\rho}_{1, j}^{2}\right)} & 0
\end{array}\right] \\
& \frac{\partial \alpha_{G S 1}}{\partial \hat{b}_{\alpha 1}}=1 \\
& \frac{\partial \delta_{G S 1}}{\partial \hat{\boldsymbol{r}}_{r s o}}=\left[-\frac{\hat{\rho}_{1, i} \hat{\rho}_{1, k}}{\hat{\rho}_{1}^{3}\left(\sqrt{1-\frac{\hat{\rho}_{1, k}^{2}}{\hat{\rho}_{1}^{2}}}\right)}-\frac{\hat{\rho}_{1, j} \hat{\rho}_{1, k}}{\hat{\rho}_{1}^{3}\left(\sqrt{1-\frac{\hat{\rho}_{1, k}^{2}}{\hat{\rho}_{1}^{2}}}\right)} \frac{\hat{\rho}_{1}^{2}-\hat{\rho}_{1, k}^{2}}{\hat{\rho}_{1}^{3}\left(\sqrt{1-\frac{\hat{\rho}_{1, k}^{2}}{\hat{\rho}_{1}^{2}}}\right)}\right] \\
& \frac{\partial \delta_{G S 1}}{\partial \hat{b}_{\delta 1}}=1 \\
& \frac{\partial \alpha_{G S 2}}{\partial \hat{\boldsymbol{r}}_{r s o}}=\left[\begin{array}{lll}
-\frac{\hat{\rho}_{2, j}}{\left(\hat{\rho}_{2, i}^{2}+\hat{\rho}_{2, j}^{2}\right)} & \frac{\hat{\rho}_{2, i}}{\left(\hat{\rho}_{2, i}^{2}+\hat{\rho}_{2, j}^{2}\right)} & 0
\end{array}\right] \\
& \frac{\partial \alpha_{G S 2}}{\partial \hat{b}_{\alpha 2}}=1
\end{aligned}
$$




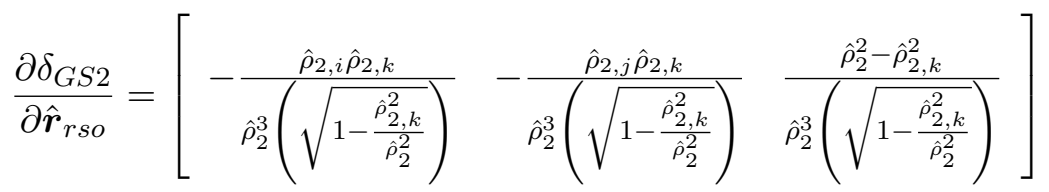

$$
\begin{aligned}
& \frac{\partial \delta_{G S 2}}{\partial \hat{b}_{\delta 2}}=1 \\
& \frac{\partial \alpha_{S S}}{\partial \hat{\boldsymbol{r}}_{r s o}}=\left[\begin{array}{lll}
-\frac{\hat{\rho}_{S S, j}}{\left(\hat{\rho}_{S S, i}^{2}+\hat{\rho}_{S S, j}^{2}\right)} & \frac{\hat{\rho}_{S S, i}}{\left(\hat{\rho}_{S S, i}^{2}+\hat{\rho}_{S S, j}^{2}\right)} & 0
\end{array}\right] \\
& \frac{\partial \alpha_{S S}}{\partial \hat{\boldsymbol{r}}_{s s}}=\left[\begin{array}{lll}
-\frac{\hat{\rho}_{S S, j}}{\left(\hat{\rho}_{S S, i}^{2}+\hat{\rho}_{S S, j}^{2}\right)} & \frac{\hat{\rho}_{S S, i}}{\left(\hat{\rho}_{S S, i}^{2}+\hat{\rho}_{S S, j}^{2}\right)} & 0
\end{array}\right]\left(-I_{3 x 3}\right) \\
& \frac{\partial \alpha_{S S}}{\partial \hat{b}_{\alpha, s s}}=1
\end{aligned}
$$

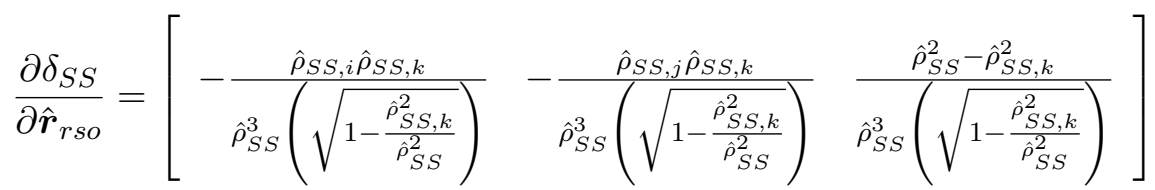

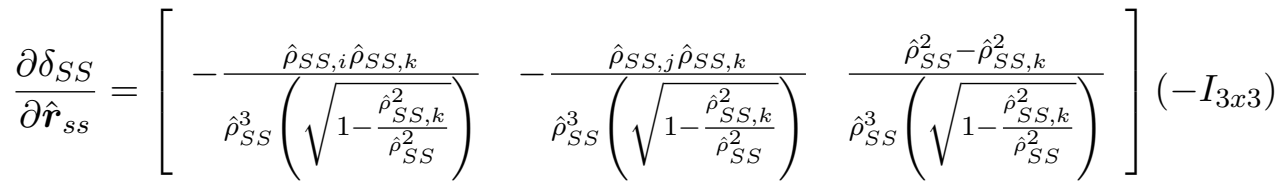

$$
\begin{aligned}
& \frac{\partial \delta_{S S}}{\partial \hat{b}_{\delta, s s}}=1
\end{aligned}
$$

The $\hat{R}_{v}$ matrix consists of four separate matrices, each supporting one of the measurement sources. When a particular measurement source is used to obtain a measurement, the $\hat{R}_{v}$ matrix corresponding to that source is used.

$$
\begin{aligned}
& \hat{R}_{v, 1}=\left[\begin{array}{cc}
\sigma_{\alpha 1}^{2} & 0 \\
0 & \sigma_{\delta 1}^{2}
\end{array}\right] \\
& \hat{R}_{v, 2}=\left[\begin{array}{cc}
\sigma_{\alpha 2}^{2} & 0 \\
0 & \sigma_{\delta 2}^{2}
\end{array}\right]
\end{aligned}
$$




$$
\begin{gathered}
\hat{R}_{v, s s}=\left[\begin{array}{cc}
\sigma_{\alpha, s s}^{2} & 0 \\
0 & \sigma_{\delta, s s}^{2}
\end{array}\right] \\
\hat{R}_{v, g p s}=\left[\begin{array}{cc}
\sigma_{g p s, r}^{2} & 0 \\
0 & \sigma_{g p s, v}^{2}
\end{array}\right]
\end{gathered}
$$

\subsection{Summary}

This chapter provided all the dynamics and equations necessary to build the Extended Kalman Filter for the RSO estimation problem. This EKF is used in the Monte Carlo analysis as well as a fundamental piece of building the LinCov analysis tool. 


\section{CHAPTER 6 \\ MONTE CARLO SIMULATION-PARAMETER VALUES}

\subsection{Overview}

This chapter provides the values and rationale used for the uncertainty parameters of the RSO estimation problem. Tables 6.1 - 6.4 at the end of the chapter summarize all values.

\subsection{RSO and SS Initial Position and Velocity Uncertainties}

The RSO and SS low, nominal, and high initial position error values were chosen to represent varying levels of knowledge of the position of these objects, in order to analyze this effect in the results. The $1-\sigma$ uncertainties in altitude, $\sigma_{h}$, downrange, $\sigma_{d}$, and crosstrack, $\sigma_{c}$, are shown in Table 6.1. The corresponding downrange, crosstrack, and altitude rates were determined as follows [30]

$$
\begin{gathered}
\dot{\sigma}_{d}=\omega_{0} \sigma_{h} \\
\dot{\sigma}_{c}=\omega_{0} \sigma_{c} \\
\dot{\sigma}_{h}=\omega_{0} \sigma_{d}
\end{gathered}
$$

where $\omega_{0}$ is the orbital rate.

The high initial position error of the RSO was originally chosen to be $100 \mathrm{~km}$ downrange, $20 \mathrm{~km}$ crosstrack, and $20 \mathrm{~km}$ altitude, 1-sigma. During Monte Carlo runs of this error level divergence occurred within the first week of analysis. The source of this divergence was determined to be non-linearities within the simulation. A second order EKF was attempted but did not fix the divergence issue. This remains an area of future analysis. The

divergence issue did not occur with lower initial position errors of the RSO, therefore the high initial position error of the RSO was reduced to $20 \mathrm{~km}$ downrange, $4 \mathrm{~km}$ crosstrack, 
and $4 \mathrm{~km}$ altitude, 1-sigma.

\subsection{Ground Station and SS Biases and Noise}

The ground station biases and noise nominal values are taken from Vallado's Fundamentals of Astrodynamics and Applications [1]. The high bias values were chosen as twice the nominal value. The low bias values come from Contributions of the GEODSS System to Catalog Maintenance [31] which collected data from observations using the GEODSS system to perform calibration.

The low space sensor biases and noise were chosen to be of the same magnitude of the nominal ground sensor values, and the nominal and high were each increased an order of magnitude from low. This means the space sensor performs less accurately than the ground stations regardless of whether the low, nominal, or high conditions are being analyzed.

\subsection{Area-to-Mass Parameters}

The area-to-mass ratio of the RSO and the space sensor are important parameters in determining the effects of solar radiation pressure. The initial mean value of the area-tomass ratio, $A_{\text {mean }}$, for the RSO is set to the order of the area-to-mass ratio of an intact spacecraft, $0.02 \mathrm{~m}^{2} / \mathrm{kg}[32]$.

The initial value of the area-to-mass ratio for the space sensor was determined by using the specifications for a cubesat [33]. A $1 \mathrm{U}$ cubesat has a mass of $1.33 \mathrm{~kg} / \mathrm{U}$ and each side of the cube is $10 \mathrm{~cm}$ in length. For this analysis, a $6 \mathrm{U}$ cubesat is used as the space sensor. The large side of a $6 \mathrm{U}$ cubesat is $30 \mathrm{~cm}$ in length and $20 \mathrm{~cm}$ in width, giving an area of 0.06 $\mathrm{m}^{2}$. The mass of the $6 \mathrm{U}$ cubesat is $7.98 \mathrm{~kg}$. Therefore, the area-to-mass ratio of the large side of a $6 \mathrm{U}$ cubesat is set at $0.00752 \mathrm{~m}^{2} / \mathrm{kg}$. The small side of a $6 \mathrm{U}$ cubesat is $30 \mathrm{~cm}$ in

length and $10 \mathrm{~cm}$ in width, giving an area of $0.03 \mathrm{~m}^{2}$. This results in an area-to-mass ratio of $0.00376 \mathrm{~m}^{2} / \mathrm{kg}$ for the small side of a $6 \mathrm{U}$ cubesat. The initial mean value of the space sensor area-to-mass ratio is set at the average value of the small side and large side, 0.0056 $\mathrm{m}^{2} / \mathrm{kg}$. 
The initial standard deviation of the area-to-mass ratio, for both the RSO and the space sensor, can be set at two different $\sigma_{A}$ values, low and nominal. These values represent the how well the initial area-to-mass ratio is known. The low value represents good knowledge of the initial area-to-mass ratio; therefore a value of $1 \%$ of the initial mean area-to-mass ratio is used. For the RSO, the low value is $\sigma_{A}=2 \times 10^{-4} \mathrm{~m}^{2} / \mathrm{kg}$. For the space sensor, the low value is $\sigma_{A}=5.6 \times 10^{-5} \mathrm{~m}^{2} / \mathrm{kg}$. The RSO nominal value is chosen so that it represents a lack of knowledge of the current configuration of the satellite with respect to the Sun. A value of $50 \%$ of the initial mean area-to-mass ratio is used for the initial 3-sigma value. For the RSO, the nominal initial 1-sigma value is set at $\sigma_{A}=3.3 \times 10^{-3} \mathrm{~m}^{2} / \mathrm{kg}$. For the space sensor, it is assumed that the area-to-mass ratio is always well known, therefore the nominal value is the same as the low value, $\sigma_{A}=5.6 \times 10^{-5} \mathrm{~m}^{2} / \mathrm{kg}$.

Low and nominal values for the strength of the random walk white noise, $Q$, were also determined. The low value for the RSO represents that the satellite is holding a near constant orientation with respect to the Sun. The nominal value for the RSO represents a satellite rotating with respect to the Sun. For the space sensor only a low value of the random walk white noise is calculated which is used for all analysis. This is to represent that the area-to-mass ratio of the space sensor is always well known.

The following relationship is used to determine the values of the strength of the random walk white noise:

$$
\sigma_{R W}^{2}=\sigma_{A}^{2}+Q t_{f}
$$

where $\sigma_{R W}^{2}$ is the variance of the random walk at the final time, $\sigma_{A_{r s o}}^{2}$ is the variance of the initial area-to-mass ratio, and $t_{f}$ is the final time, 7 days or 604800 seconds.

For the low value for $Q_{A_{r s o}}$ and the value for $Q_{A_{s s}}, 4.2 \%$ of the initial area to mass ratio is used for the final 3-sigma value of the random walk. Using this 3 -sigma value for the random walk, the resulting noise values are $Q_{A_{\text {rso }}}=6.61 \times 10^{-14} \mathrm{~m}^{4} /\left(\mathrm{kg}^{2} \mathrm{~s}\right)$ for the RSO and a value of $Q_{A_{s s}}=5.19 \times 10^{-15} \mathrm{~m}^{4} /\left(\mathrm{kg}^{2} \mathrm{~s}\right)$ for the space sensor. 
For the nominal value of $Q_{A_{r s o}}$, care must be taken to ensure the value of the area-tomass ratio does not become negative. To ensure the value does not go negative within the simulation time, a value of $70.7 \%$ of the initial area to mass ratio, or $\frac{\sqrt{2}}{2}\left(A_{\text {mean }}\right)$, is used for the final 3-sigma value of the random walk.

Using this 3-sigma value for the random walk and solving for $Q_{A_{r s o}}$ in Equation 6.4 gives:

$$
Q_{A_{r s o}}=\frac{\sigma_{R W}^{2}-\sigma_{A_{r s o}}^{2}}{t_{f}}=\frac{\left(\frac{\sqrt{2}}{6}\left(A_{\text {mean }}\right)\right)^{2}-\left(\sigma_{A_{r s o}}^{2}\right)}{t_{f}}
$$

where $\sigma_{A_{r s o}}^{2}$ is the variance of the initial area-to-mass ratio, and $t_{f}$ is the final time, which is 7 days or 604800 seconds.

For the RSO, the nominal value of $Q_{A_{r s o}}$ is determined through use of the initial areato-mass 1-sigma value of $\sigma_{A}=3.3 \times 10^{-3} \mathrm{~m}^{2} / \mathrm{kg}$ in Eq. 6.5 , giving a nominal value of $Q_{A_{r s o}}$ $=1.84 \times 10^{-11} \mathrm{~m}^{4} /\left(\mathrm{kg}^{2} \mathrm{~s}\right)$.

The initial standard deviations and random walk white noise strength values are combined to create three cases to be analyzed, low, nominal, and high. For the RSO, the low case represents high knowledge of the initial area-to-mass ratio and a near constant orientation with respect to the Sun. Therefore it utilizes the low initial standard deviation and low white noise strength. The nominal case represents lack of knowledge of the current attitude of the satellite with respect to the Sun, but a near constant orientation with respect to the Sun. It utilizes the nominal initial standard deviation and the low white noise strength. The high case represents lack of knowledge of the current orientation of the satellite with respect to the Sun and a rotating satellite (nadir pointing or other). It utilizes the nominal initial standard deviation and the nominal white noise strength. For the space sensor, the same initial condition and white noise strength is used for all levels of analysis: low, nominal, and high. 


\subsection{Disturbance Accelerations}

Higher-order gravity terms above $J_{2,2}$ have an affect on the geosynchronous satellite and the space sensor and are modeled with an ECRV.

In order to model the higher-order gravitational effects, representational values of the strength of the white noise and the time constant were determined.

In the truth model, the nominal RSO GEO orbit was propagated using a full $18 \times 18$ gravitational model. Next, the value of the acceleration due to a $J_{2,2}$ gravitational model at each location in the orbit was determined. The difference between the full $18 \times 18$ gravitational model perturbation and the $J_{2,2}$ gravitational model perturbation was taken to define the gravitational effects above $J_{2,2}$. The 3 -sigma bounds and time constant of the disturbance acceleration were determined from these results. The inertial x component difference is shown in Figure 6.1. By analyzing this plot, the time constant is set to 43200 seconds. The largest difference, the inertial z component difference, is shown in Figure 6.2 , the 3 -sigma bounds were taken as the maximum $3 \times 10^{-9} \mathrm{~m} / \mathrm{s}^{2}$, this gives a 1 -sigma value of $1 \times 10^{-9} \mathrm{~m} / \mathrm{s}^{2}$. This value was then used for the ECRV to ensure it encompassed the position errors caused by gravitational accelerations above $J_{2,2}$. Figure 3 shows these position errors. In Figure 6.3, it can be seen that the resulting ECRV position covariance does not fully encompass the errors; therefore the ECRV is tuned to a sigma value of 1.73 x $10^{-9} \mathrm{~m} / \mathrm{s}^{2}$. Figure 6.4 shows the revised 1-sigma position covariance encompassing the position error. 


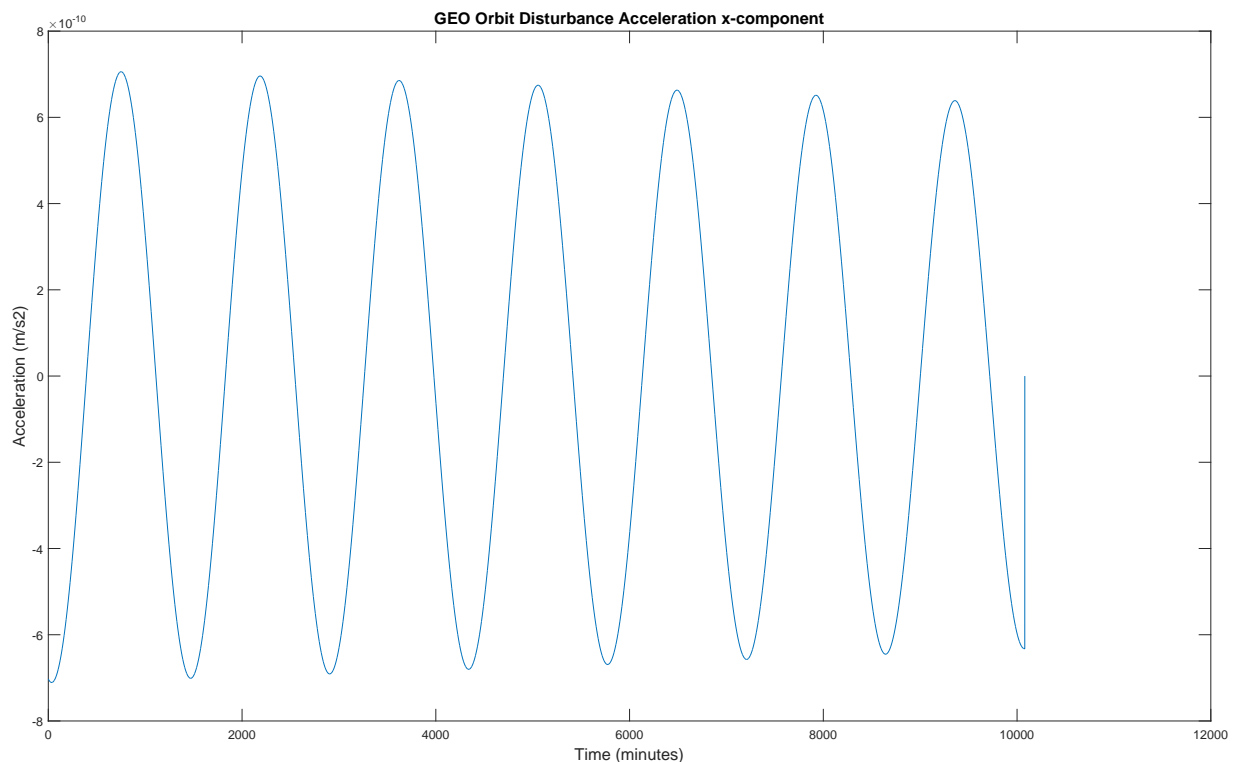

Fig. 6.1: RSO GEO orbit inertial x-component of higher-order disturbance acceleration

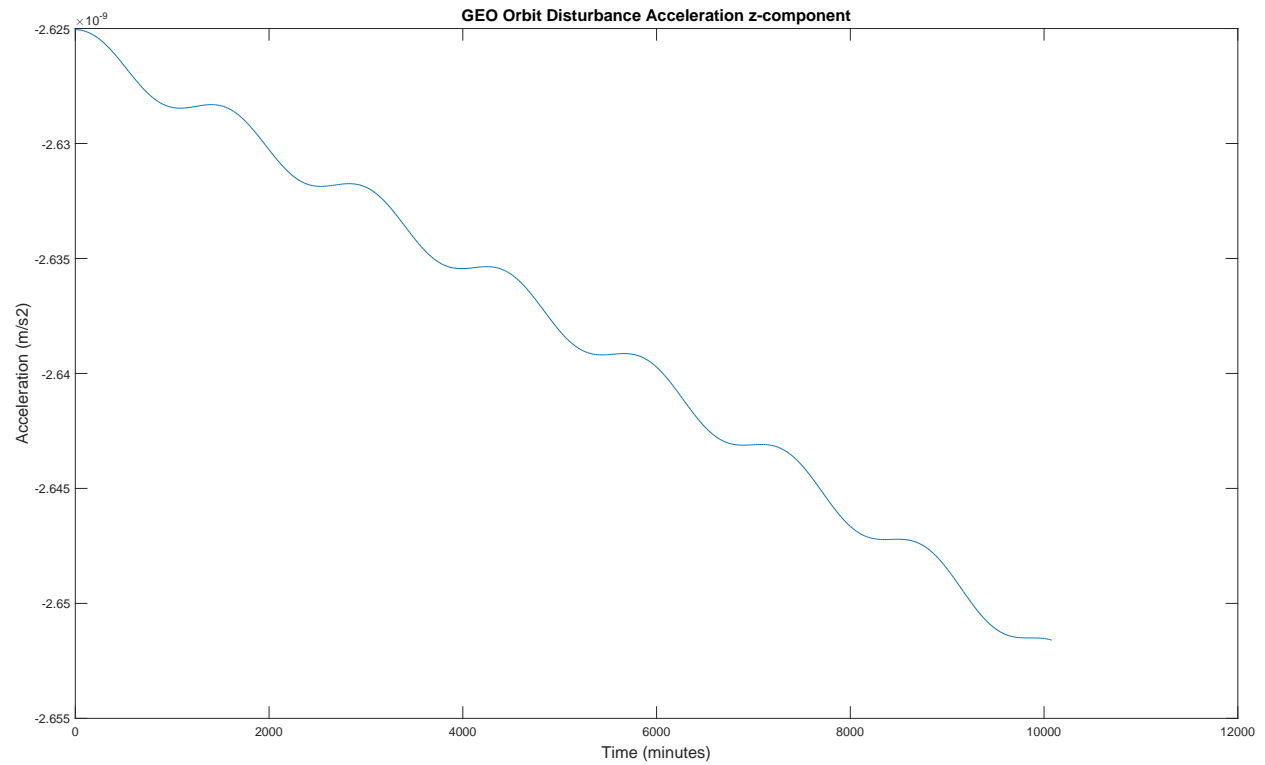

Fig. 6.2: RSO GEO orbit inertial z-component of higher-order disturbance acceleration 

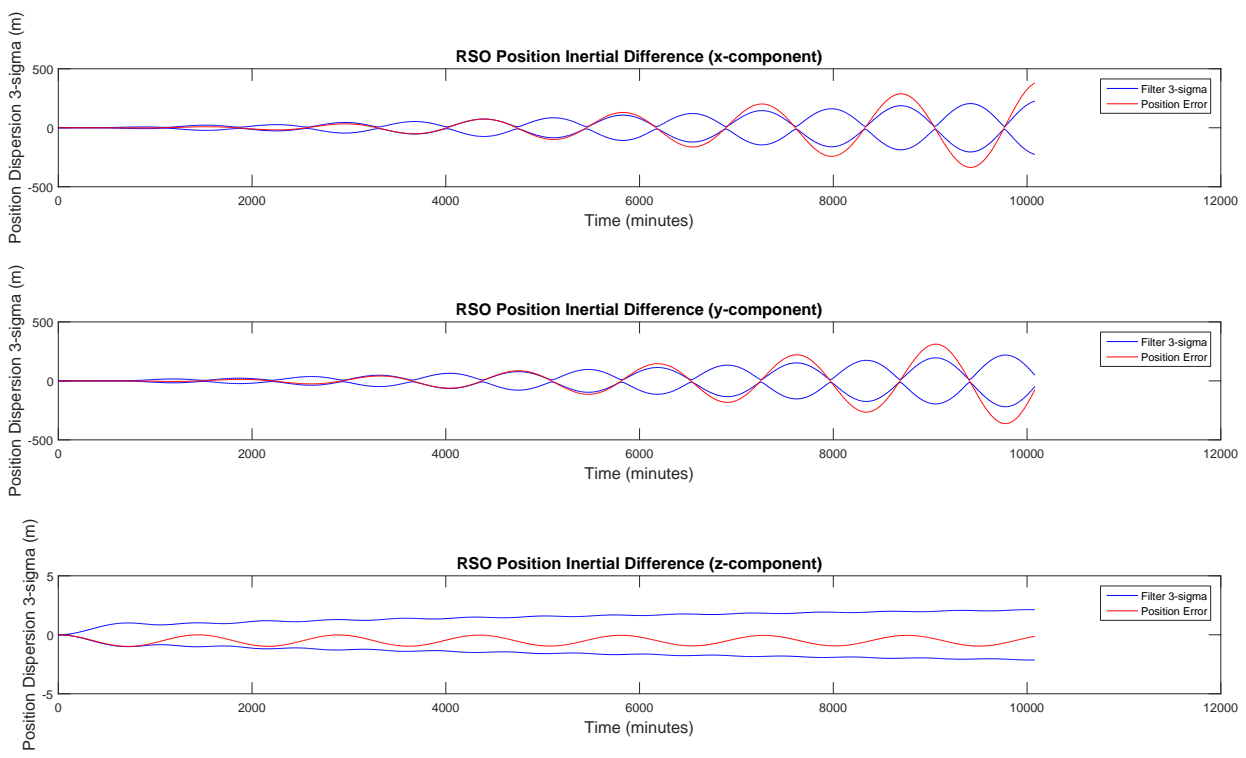

Fig. 6.3: RSO GEO orbit original ECRV 3- $\sigma$ position error
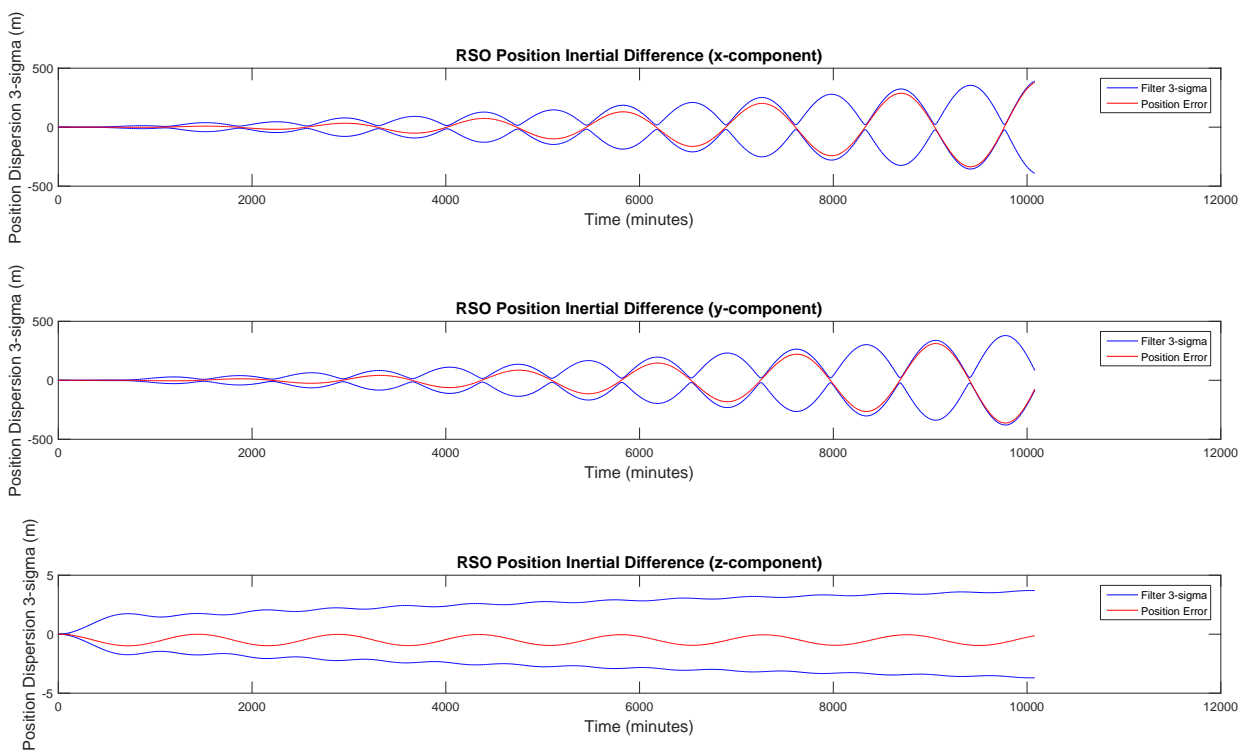

Fig. 6.4: RSO GEO orbit revised ECRV 3- $\sigma$ position error 
The same approach taken for the RSO GEO orbit was applied for each of the space sensor orbits. For the low circular space sensor orbit, Fig 6.5 shows the difference between the full $18 \times 18$ gravitational model and the $J_{2,2}$ gravitational model accelerations for the low circular space sensor orbit. Based on these results, the time constant was set to 18900 seconds and the one sigma value set to $1.67 \times 10^{-8} \mathrm{~m} / \mathrm{s}^{2}$. This value used within the ECRV encompassed the low circular SS orbit position errors, as shown in Figure 6.6.

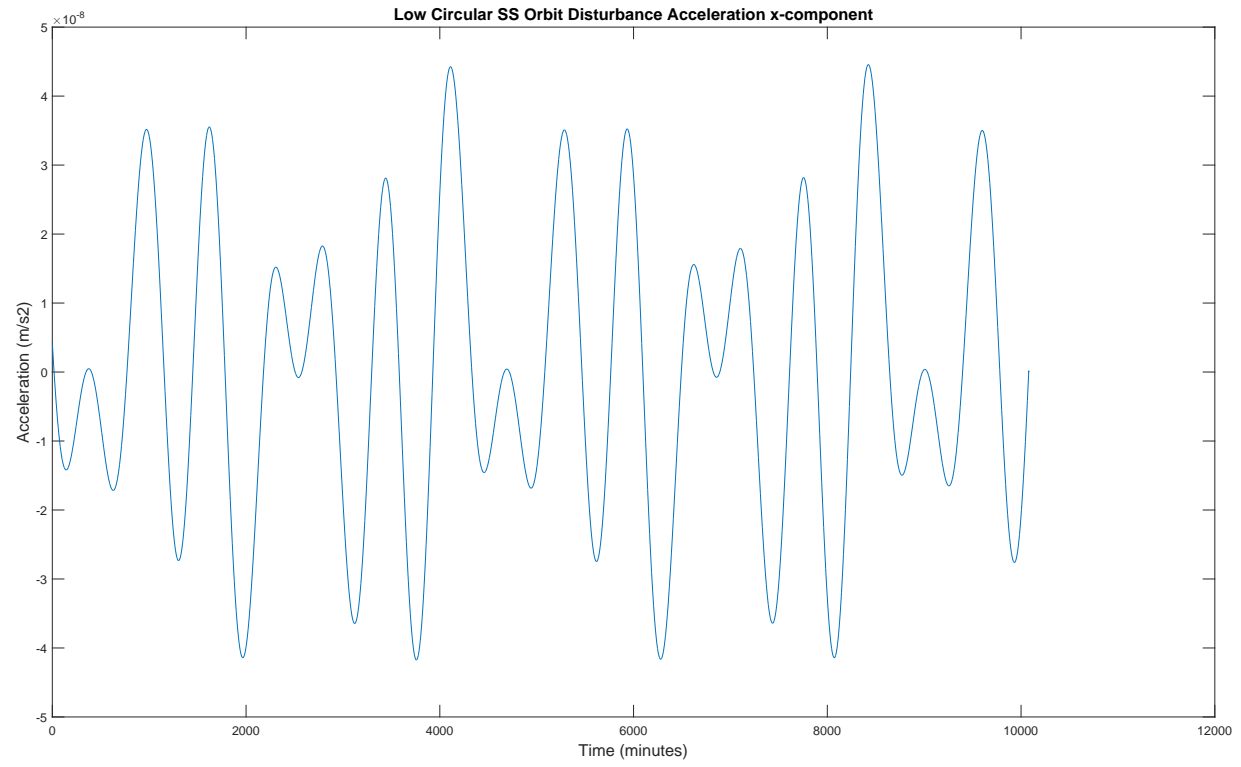

Fig. 6.5: Low Circular x-component higher-order disturbance acceleration 


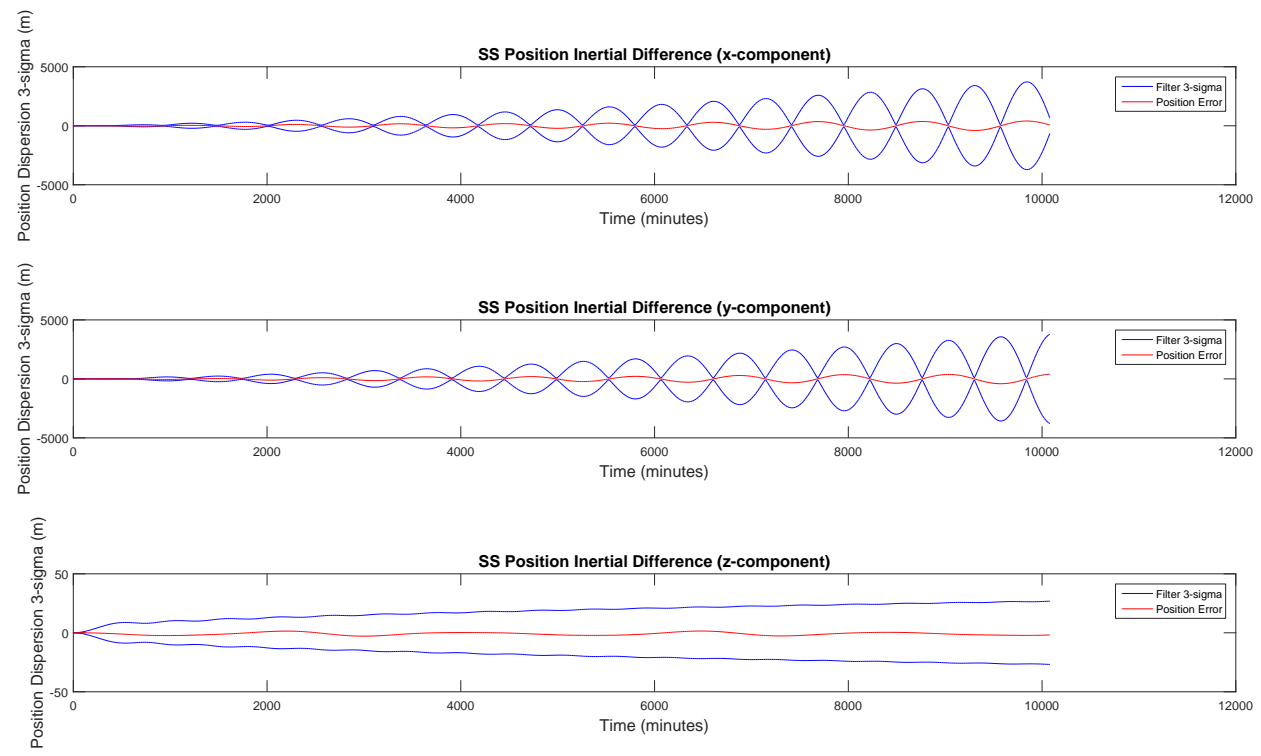

Fig. 6.6: Low Circular ECRV 3- $\sigma$ position error

For the high circular space sensor orbit, Fig 6.7 shows the difference between the full $18 \times 18$ gravitational model and the $J_{2,2}$ gravitational model accelerations for the high circular space sensor orbit. Based on these results, the time constant was set to 33600 seconds and the one sigma value set to $5 \times 10^{-9} \mathrm{~m} / \mathrm{s}^{2}$. This value used within the ECRV did not encompass the geosynchronous position errors, as shown in Figure 6.8. Therefore, the ECRV was further tuned to a sigma value of $8.66 \times 10^{-9} \mathrm{~m} / \mathrm{s}^{2}$. The resulting high circular SS orbit position covariance is shown in Fig 6.9. 


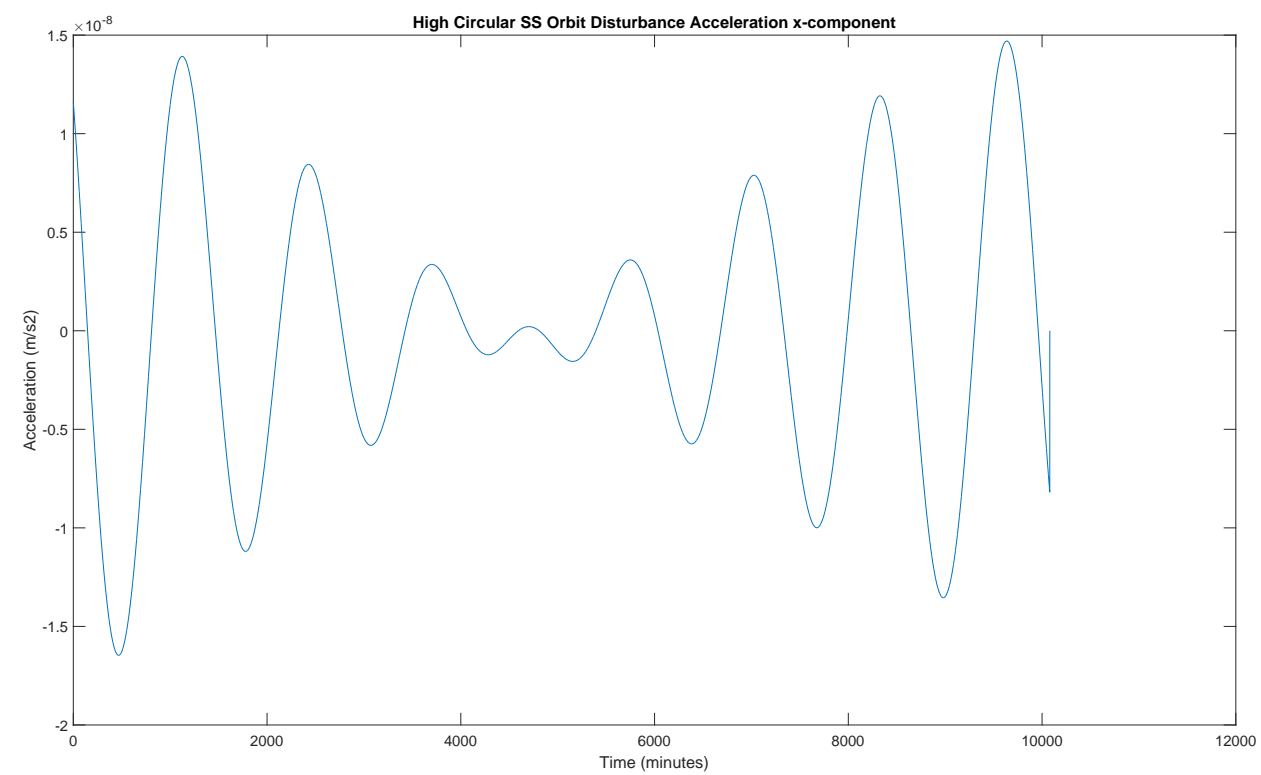

Fig. 6.7: High Circular x-component higher-order disturbance acceleration
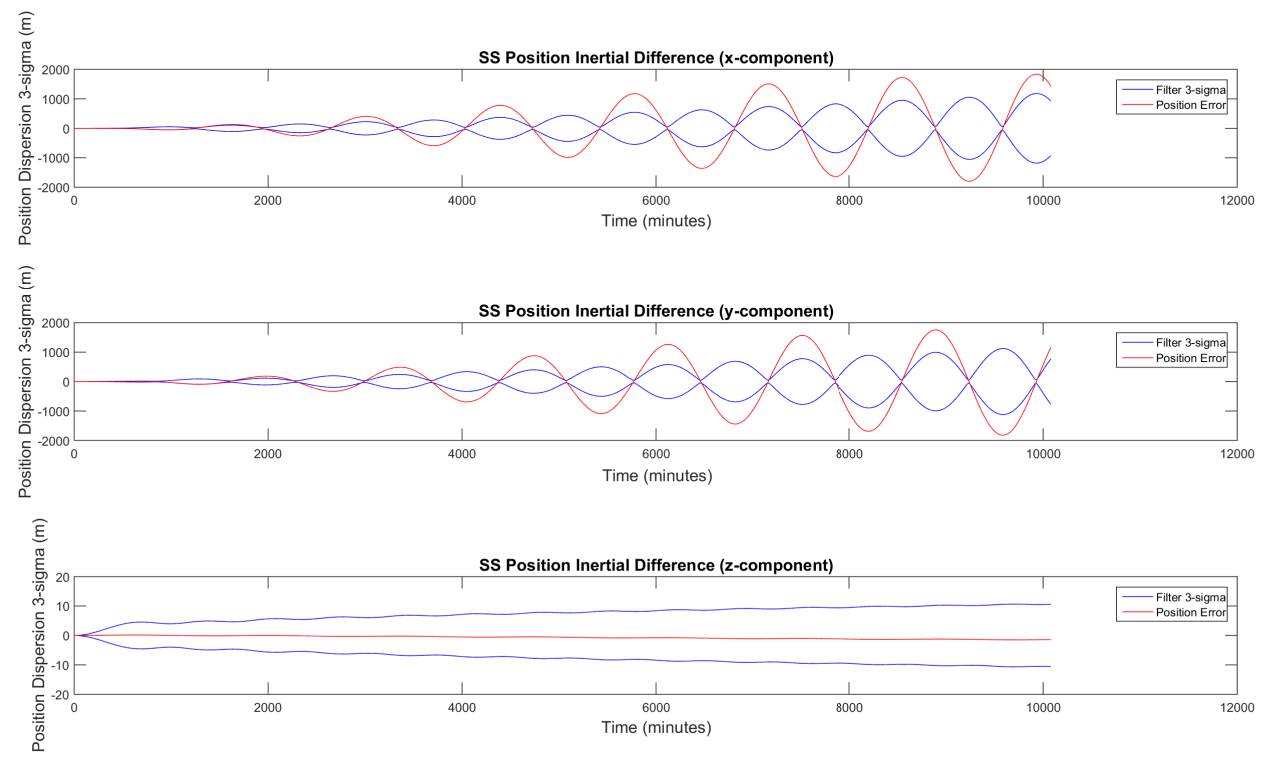

Fig. 6.8: High Circular Original ECRV 3- $\sigma$ position error 

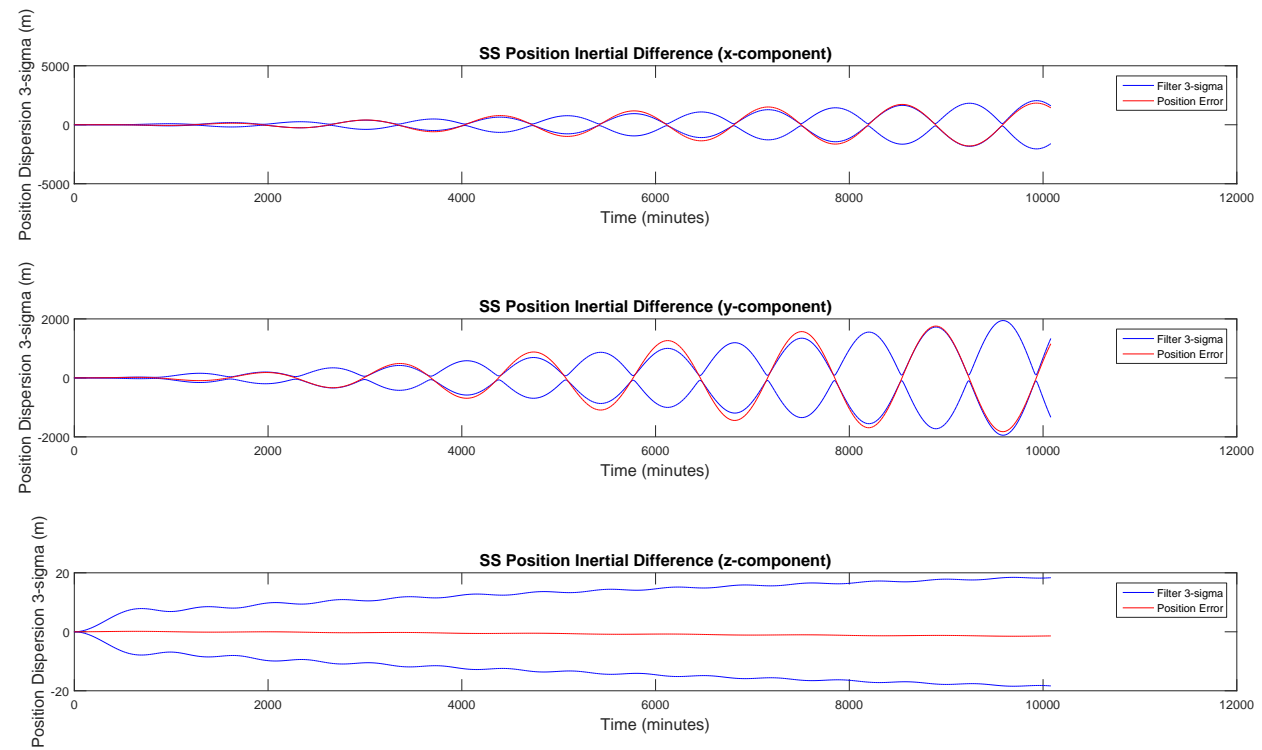

Fig. 6.9: High Circular Revised ECRV 3- $\sigma$ position error

For the elliptical space sensor orbit, Fig. 6.10 shows the difference between the full $18 \times 18$ gravitational model and the $J_{2,2}$ gravitational model accelerations for the elliptical space sensor orbit. Based on these results, the time constant was set to 23262 seconds and the one sigma value set to $1.67 \times 10^{-8} \mathrm{~m} / \mathrm{s}^{2}$. This value used within the ECRV encompassed the elliptical SS orbit position errors, as shown in Fig. 6.11. 


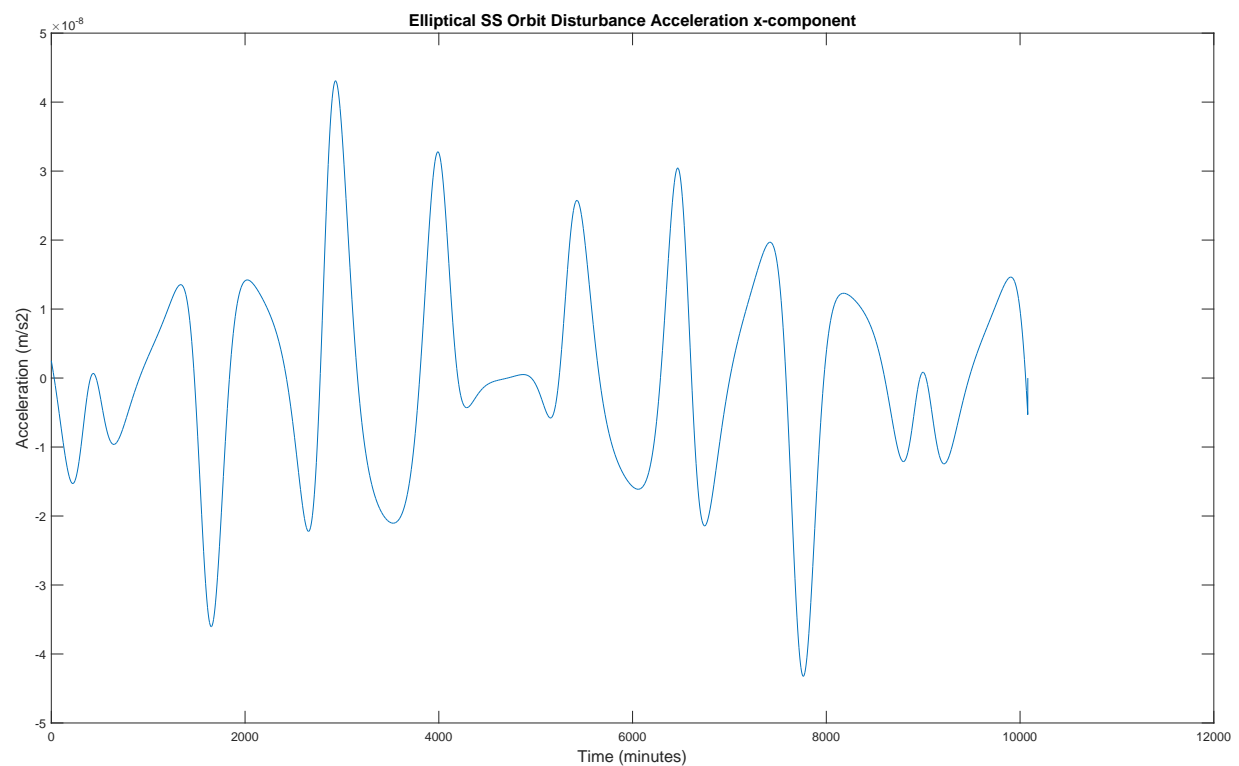

Fig. 6.10: Elliptical x-component higher-order disturbance acceleration

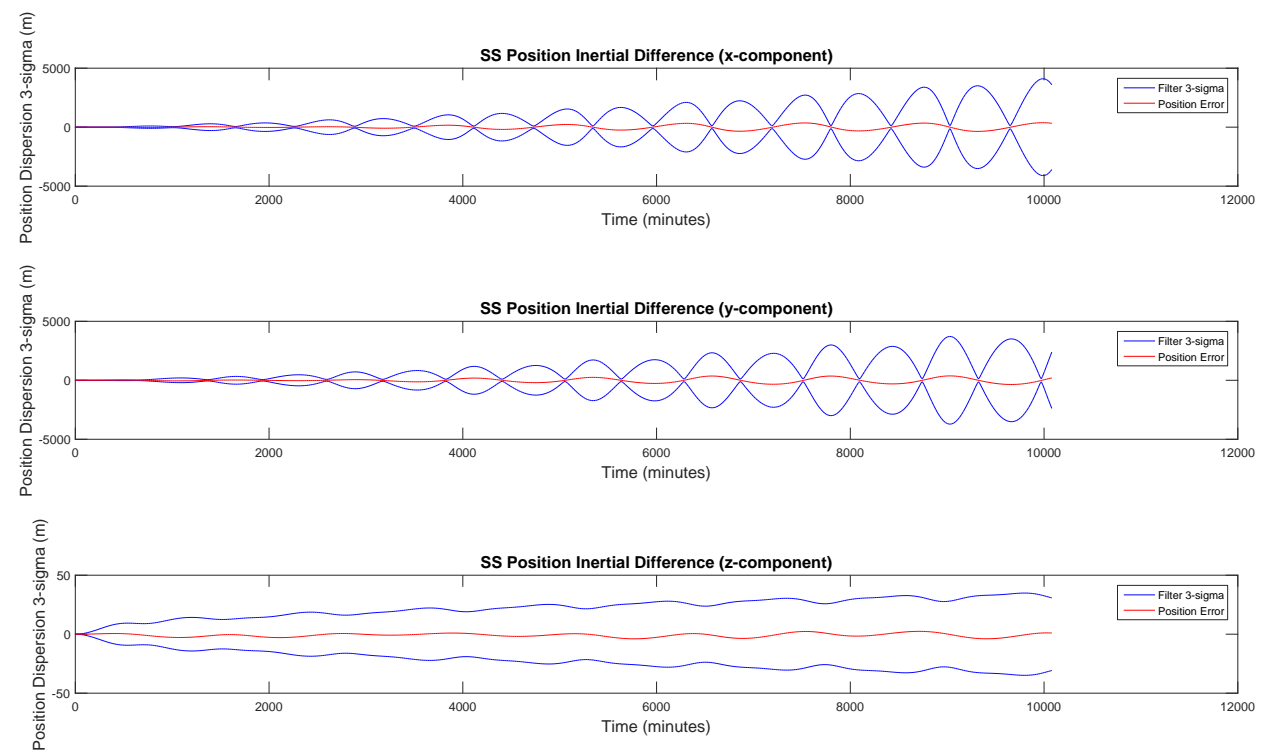

Fig. 6.11: Elliptical ECRV 3- $\sigma$ position error 


\subsection{Random Accelerations}

To account for the accelerations that are not incorporated in the RSO dynamics, such as the effects from other planets, process noise is included on the RSO. The process noise values are set at low, nominal, and high levels. These levels were chosen to represent a position error growth of 10, 100, and 1000 meters per day. The values of process noise strength that correspond to these low, nominal, and high position errors are $1 \times 10^{-14}$ $\mathrm{m}^{2} / \mathrm{s}^{3}, 1 \times 10^{-12} \mathrm{~m}^{2} / \mathrm{s}^{3}$, and $1 \times 10^{-10} \mathrm{~m}^{2} / \mathrm{s}^{3}$ respectively.

To account for the accelerations that are not incorporated in the SS dynamics, process noise is also included on the space sensor. These levels were chosen to represent a position error growth of 1, 10, and 100 meters per day. The values of process noise strength that

correspond to these low, nominal, and high position errors are $1 \times 10^{-16} \mathrm{~m}^{2} / \mathrm{s}^{3}, 1 \times 10^{-14}$ $\mathrm{m}^{2} / \mathrm{s}^{3}$, and $1 \times 10^{-12} \mathrm{~m}^{2} / \mathrm{s}^{3}$ respectively for all three space sensor orbits, low circular, high circular, and elliptical.

\subsection{Summary}

All critical parameters, their symbols, and their values used for analysis and results are provided in Table 6.1 through Table 6.4 . 
Table 6.1: RSO Initial State Vector Uncertainties

\begin{tabular}{|c|c|c|c|c|}
\hline & & \multicolumn{3}{|c|}{ Initial Condition $1-\sigma$ Values } \\
\hline Component & Symbol & Low Value & Nominal Value & High Value \\
\hline RSO Down-Range position (km) & $\sigma_{d_{r s o}}$ & 1 & 10 & 20 \\
\hline RSO Cross-Track position (km) & $\sigma_{c_{r s o}}$ & 0.2 & 2 & 4 \\
\hline RSO Altitude position (km) & $\sigma_{h_{r s o}}$ & 0.2 & 2 & 4 \\
\hline RSO Down-Range velocity $(\mathrm{m} / \mathrm{s})$ & $\sigma_{\dot{d}_{r s o}}$ & $1.45 \times 10^{-2}$ & 0.145 & 0.291 \\
\hline RSO Cross-Track velocity $(\mathrm{m} / \mathrm{s})$ & $\sigma_{\dot{c}_{r s o}}$ & $1.45 \times 10^{-2}$ & 0.145 & 0.291 \\
\hline RSO Altitude velocity (m/s) & $\sigma_{\dot{h}_{r s o}}$ & $7.27 \times 10^{-2}$ & 0.727 & 1.45 \\
\hline $\begin{array}{l}\text { RSO Area to Mass }\left(\mathrm{m}^{2} / \mathrm{kg}\right) \\
\text { Mean Value }=2 \times 10^{-2}\end{array}$ & $\sigma_{A_{\text {rso }}}$ & $2 \times 10^{-4}$ & $3.3 \times 10^{-3}$ & $3.3 \times 10^{-3}$ \\
\hline $\begin{array}{l}\text { RSO Reflectivity Coeff. } \\
\qquad \text { Mean Value }=1\end{array}$ & $\sigma_{C_{r s o}}$ & 0 & 0 & 0 \\
\hline
\end{tabular}


Table 6.2: Space Sensor Initial State Vector Uncertainties

\begin{tabular}{|c|c|c|c|c|}
\hline \multirow[b]{2}{*}{ Component } & \multirow[b]{2}{*}{ Symbol } & \multicolumn{3}{|c|}{ Initial Condition $1-\sigma$ Values } \\
\hline & & Low Value & Nominal Value & High Value \\
\hline SS Down-Range position $(\mathrm{km})$ & $\sigma_{d_{s s}}$ & 0.1 & 1 & 10 \\
\hline SS Cross-Track position (km) & $\sigma_{c_{s s}}$ & 0.02 & 0.2 & 2 \\
\hline SS Altitude position (km) & $\sigma_{h_{s s}}$ & 0.02 & 0.2 & 2 \\
\hline SS Down-Range velocity $(\mathrm{m} / \mathrm{s})$ & $\sigma_{\dot{d}_{s s}}$ & $2.2 \times 10^{-3}$ & $2.2 \times 10^{-2}$ & 0.22 \\
\hline SS Cross-Track velocity (m/s) & $\sigma_{\dot{c}_{s s}}$ & $2.2 \times 10^{-3}$ & $2.2 \times 10^{-2}$ & 0.22 \\
\hline SS Altitude velocity (m/s) & $\sigma_{\dot{h}_{s s}}$ & $1.1 \times 10^{-2}$ & 0.11 & 1.1 \\
\hline $\begin{array}{l}\text { SS Area to Mass }\left(\mathrm{m}^{2} / \mathrm{kg}\right) \\
\text { Mean Value }=5.6 \times 10^{-3}\end{array}$ & $\sigma_{A_{s s}}$ & $5.6 \times 10^{-5}$ & $5.6 \times 10^{-5}$ & $5.6 \times 10^{-5}$ \\
\hline $\begin{array}{l}\text { SS Reflectivity Coeff. } \\
\qquad \text { Mean Value }=1\end{array}$ & $\sigma_{C_{s s}}$ & 0 & 0 & 0 \\
\hline
\end{tabular}


Table 6.3: Measurement Errors

\begin{tabular}{|c|c|c|c|c|}
\hline & & \multicolumn{3}{|c|}{ Initial Condition $1-\sigma$ Values } \\
\hline Component & Symbol & Low Value & Nominal Value & High Value \\
\hline Ground Station 1 ra bias ( $\mu \mathrm{rad})$ & $\sigma_{b_{\alpha 1}}$ & 2.6 & 29.7 & 60 \\
\hline Ground Station 1 dec bias ( $\mu \mathrm{rad}$ ) & $\sigma_{b_{\delta 1}}$ & 0.8 & 17.5 & 35 \\
\hline Ground Station 2 ra bias ( $\mu \mathrm{rad})$ & $\sigma_{b_{\alpha 2}}$ & 2.6 & 40.1 & 80 \\
\hline Ground Station 2 dec bias ( $\mu \mathrm{rad})$ & $\sigma_{b_{\delta 2}}$ & 0.8 & 29.7 & 60 \\
\hline SS ra bias ( $\mu$ rad) & $\sigma_{b_{\alpha, s s}}$ & 50 & 500 & 5000 \\
\hline SS dec bias ( $\mu \mathrm{rad})$ & $\sigma_{b_{\delta, s s}}$ & 50 & 500 & 5000 \\
\hline Ground Station 1 ra noise ( $\mu$ rad) & $\sigma_{\eta_{\alpha 1}}$ & \multicolumn{3}{|c|}{57.6} \\
\hline Ground Station 1 dec noise ( $\mu \mathrm{rad})$ & $\sigma_{\eta_{\delta 1}}$ & \multicolumn{3}{|c|}{47.1} \\
\hline Ground Station 2 ra noise ( $\mu$ rad) & $\sigma_{\eta_{\alpha 2}}$ & \multicolumn{3}{|c|}{64.6} \\
\hline Ground Station 2 dec noise ( $\mu \mathrm{rad})$ & $\sigma_{\eta_{\delta 2}}$ & \multicolumn{3}{|c|}{52.4} \\
\hline SS ra noise $(\mu \mathrm{rad})$ & $\sigma_{\eta_{\alpha, s s}}$ & 50 & 500 & 5000 \\
\hline SS dec noise ( $\mu \mathrm{rad})$ & $\sigma_{\eta_{\delta, s s}}$ & 50 & 500 & 5000 \\
\hline GPS Position noise $(\mathrm{km})$ & $\sigma_{g p s, r}$ & 0.01 & 0.1 & 1 \\
\hline GPS Velocity noise $(\mathrm{m} / \mathrm{s})$ & $\sigma_{g p s, v}$ & 0.001 & 0.01 & 0.1 \\
\hline
\end{tabular}


Table 6.4: Other Design Parameters

\begin{tabular}{|c|c|c|c|c|}
\hline Component & Symbol & Low Value & Nominal Value & High Value \\
\hline RSO Random Accels $\left(\mathrm{m}^{2} / \mathrm{s}^{3}\right)$ & $Q_{\text {rso }}$ & $1 \times 10^{-14}$ & $1 \times 10^{-12}$ & $1 \times 10^{-10}$ \\
\hline $\begin{array}{l}\text { RSO Area to Mass Ratio } \\
\text { Noise Strength }\left(\mathrm{m}^{4} / \mathrm{kg}^{2} / \mathrm{s}\right)\end{array}$ & $Q_{A_{r s o}}$ & $6.61 \times 10^{-14}$ & $6.61 \times 10^{-14}$ & $1.84 \times 10^{-11}$ \\
\hline $\begin{array}{c}\text { RSO Dist Acceleration } \\
\text { (ECRV) Time Constant (s) }\end{array}$ & $\tau_{\text {rso }}$ & \multicolumn{3}{|c|}{43200} \\
\hline $\begin{array}{l}\text { RSO Dist Acceleration (ECRV) } \\
\text { Steady State } 1-\sigma / \text { axis }\left(\mathrm{m} / \mathrm{s}^{2}\right)\end{array}$ & $\sigma_{r s o}$ & \multicolumn{3}{|c|}{$1.73 \times 10^{-9}$} \\
\hline SS Random Accels $\left(\mathrm{m}^{2} / \mathrm{s}^{3}\right)$ & $Q_{s s}$ & $1 \times 10^{-16}$ & $1 \times 10^{-14}$ & $1 \times 10^{-12}$ \\
\hline $\begin{array}{c}\text { SS Area to Mass Ratio } \\
\text { Noise Strength }\left(\mathrm{m}^{4} / \mathrm{kg}^{2} / \mathrm{s}\right)\end{array}$ & $Q_{A_{s s}}$ & $5.19 \times 10^{-15}$ & $5.19 \times 10^{-15}$ & $5.19 \times 10^{-15}$ \\
\hline SS Dist Acceleration & & \multicolumn{3}{|c|}{33600 - High Circular Orbit } \\
\hline (ECRV) Time Constant (s) & $\tau_{s s}$ & \multicolumn{3}{|c|}{18900 - Low Circular Orbit } \\
\hline & & \multicolumn{3}{|c|}{23262 - Elliptical Orbit } \\
\hline SS Dist Acceleration (ECRV) & & \multicolumn{3}{|c|}{$8.66 \times 10^{-9}-$ High Circular Orbit } \\
\hline & $\sigma_{s s}$ & \multicolumn{3}{|c|}{$1.67 \times 10^{-8}$ - Low Circular Orbit } \\
\hline & & \multicolumn{3}{|c|}{$1.67 \times 10^{-8}$ - Elliptical Orbit } \\
\hline
\end{tabular}




\section{CHAPTER 7 \\ EXTENDED KALMAN FILTER VALIDATION}

\subsection{EKF Validation Method Overview}

The extended Kalman filter developed in Chapter 5 must be validated prior to its use in creating a LinCov analysis tool. In order to validate the EKF, a set of runs were completed in which the Monte Carlo results were compared to the EKF results, focusing on the critical analysis areas and parameters. All Monte Carlo results are bounded by confidence intervals. Each plot in the chapter shows the EKF 3-sigma value, the Monte Carlo 3-sigma value, and

the confidence interval around the Monte Carlo value. If the EKF results fall within the confidence interval bounds, the EKF is validated.

The Monte Carlo covariance is calculated as shown in Eq. 7.1 .

$$
P(t)=\frac{1}{N-1} \sum_{i=1}^{N}\left(\boldsymbol{e}(t)_{i}-M\right)\left(\boldsymbol{e}(t)_{i}-M\right)
$$

where $N$ is number of sample runs, $\boldsymbol{e}$ is the error vector, defined as $\boldsymbol{e}=\boldsymbol{x}-\hat{\boldsymbol{x}}$ and $M$ is the mean of the data, defined as: $M=\sum_{i=1}^{N} \frac{e(t)_{i}}{N}$.

The 1-sigma Monte Carlo values are determined by taking the square root of the diagonal elements of the covariance matrix. Finally, this value is multiplied by 3 to get the 3-sigma Monte Carlo value.

To determine the 3-sigma EKF values, the 1-sigma values are determined by taking the square root of the diagonal elements of the EKF covariance matrix. Then, this value is multiplied by 3 to get the 3 -sigma EKF value.

The number of sample runs for all validation results is 50 .

The confidence intervals are calculated using a chi-square distribution with N-1 degrees of freedom. The chosen confidence level is 95\%. [34] 
The first set of validation runs verify that the EKF operates correctly within the environment it was created for, a $2 \times 2$ gravity model in both the truth model and the EKF. The analysis begins by verifying that the EKF can estimate the ground station and space sensor measurement biases. Next, the SRP area-to-mass ratio for both the RSO and SS is shown to be estimated properly by the EKF. RSO and space sensor disturbance acceleration estimation is then analyzed. The validation runs are completed with an analysis consisting of nominal and high error source levels for ground station measurement only scenarios and nominal and high error source levels for each space sensor orbit. Nominal tasking profiles are used for the ground stations and space sensor.

The second set of validation runs verify that the developed EKF operates within a truth environment that incorporates an $18 \times 18$ gravity model and does not include disturbance accelerations. Each space sensor orbit is analyzed with low error source levels, using nominal tasking profiles for the ground stations and space sensor.

All figures except, Figures 7.27 and 7.29, have one sample plotted against the Monte Carlo results and confidence intervals. This was done to reduce the figure size. No information is lost because when all 50 samples are plotted they are so tightly spaced that no difference is noticed in the figures. Figures 7.27 and 7.29 are left with all 50 samples plotted because they are results from a high error level analysis and, as such, the 50 samples are spread out more in the results. These figures still verify the EKF analysis tool, but plotting one sample would not convey all the information.

\subsection{Measurement Bias Estimation}

The purpose of this analysis is to check that the EKF can estimate the measurement biases of both ground stations and the space sensor. The state errors turned on for this analysis are the biases on each measurement source, set to the nominal error source level. The low circular space sensor orbit is used for this analysis and the space sensor accuracy is set to its most accurate level. Ground station measurement noise is turned on. It can be seen, in Figures 7.1 through 7.3 that the EKF is capable of estimating the biases of each measurement source. With each measurement taken, bias estimate accuracy is improved. 
The EKF results fall within the Monte Carlo confidence intervals.
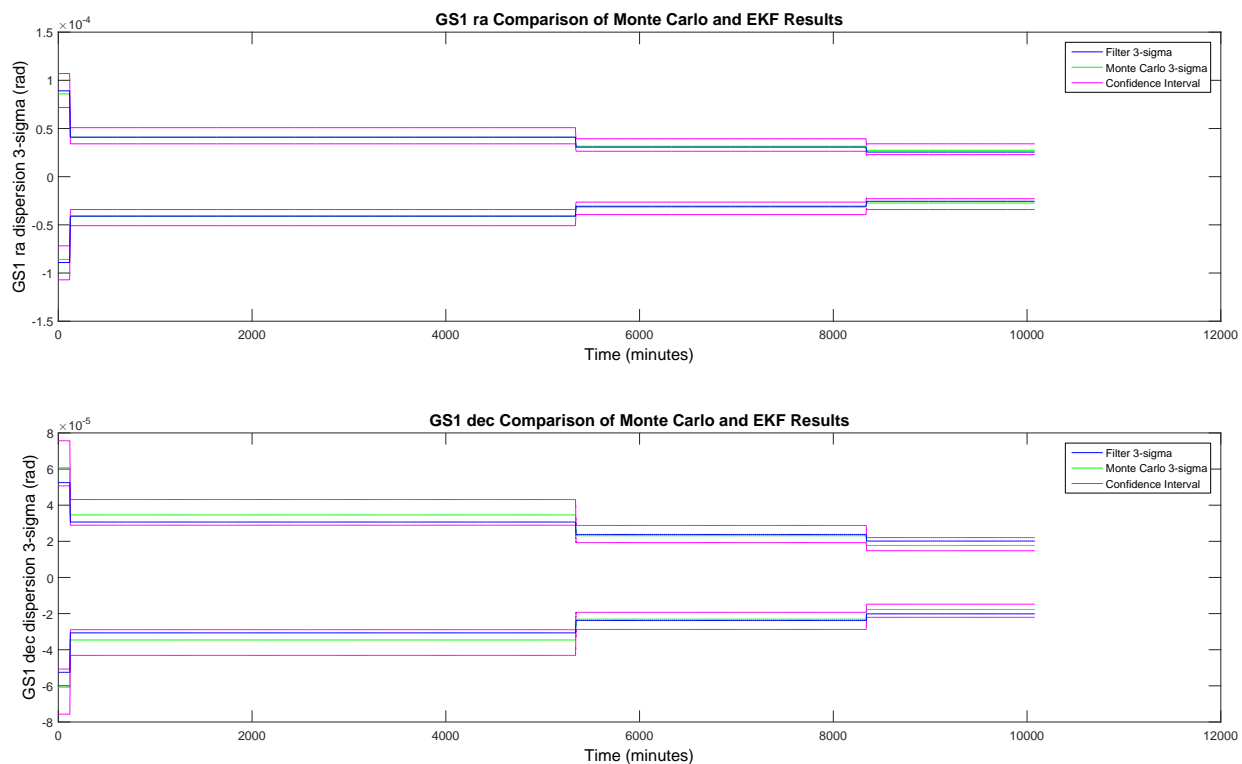

Fig. 7.1: GS1 Measurement Bias, EKF Validation, Estimation Errors
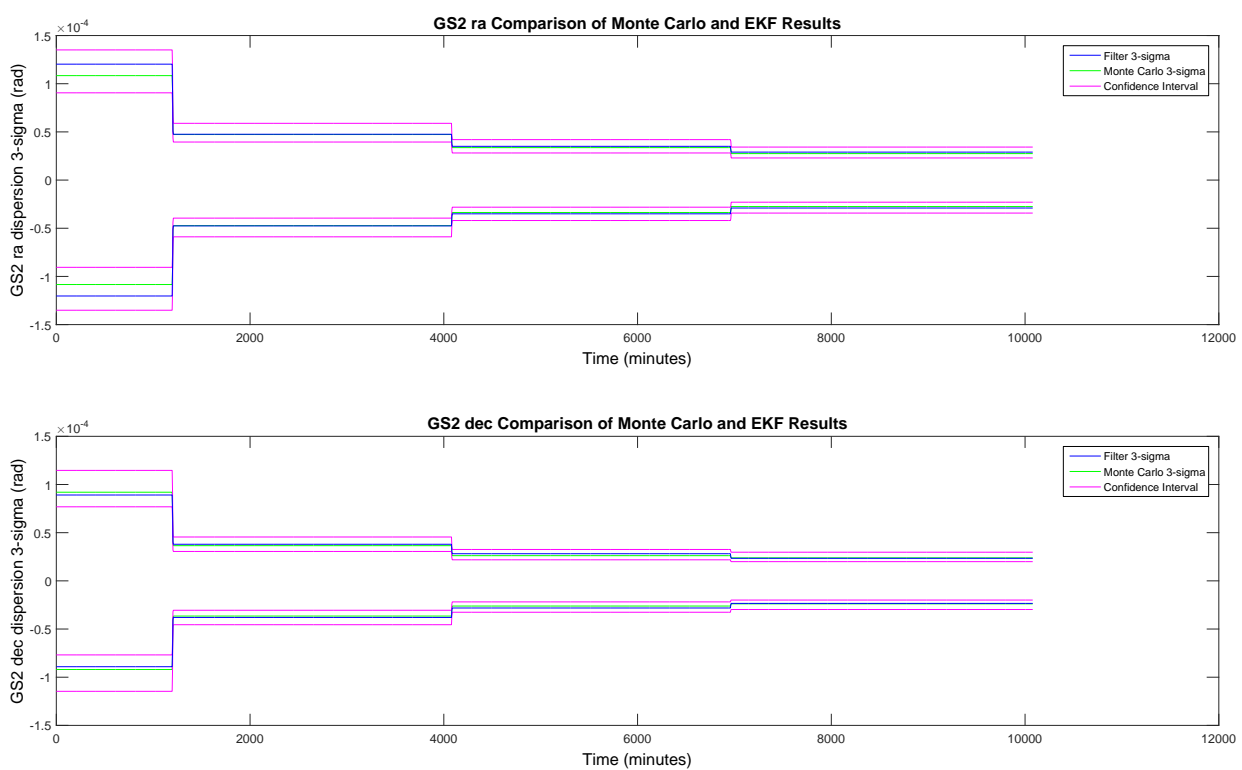

Fig. 7.2: GS2 Measurement Bias, EKF Validation, Estimation Errors 

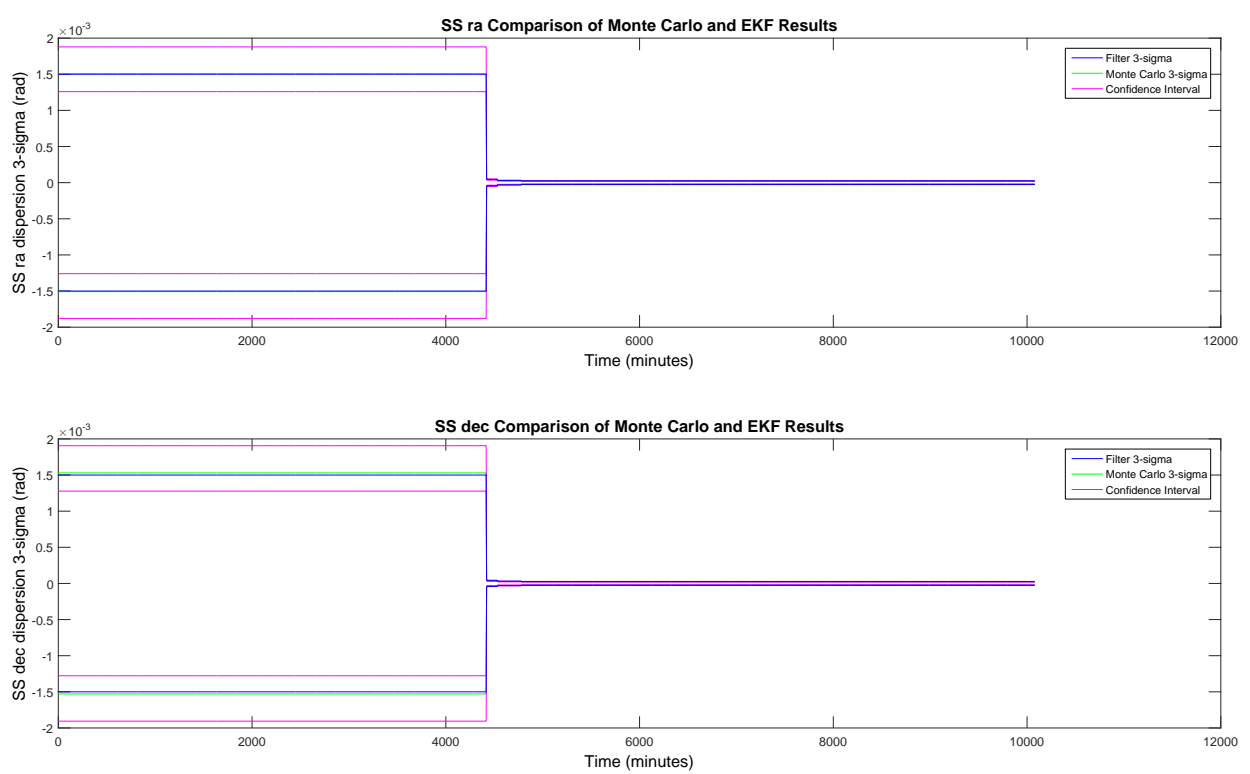

Fig. 7.3: Space Sensor Measurement Bias, EKF Validation, Estimation Errors

\subsection{SRP Estimation}

The purpose of this analysis is to check that the EKF can estimate the area-to-mass ratio of both the RSO and the space sensor. The RSO area-to-mass ratio is set to its highest initial condition error level, and the strength of the random walk noise, $Q_{A_{\text {rso }}}$, is set to its lowest error level. This simulates a large uncertainty in the value of the area-tomass ratio, but a fairly constant value over time. The space sensor area-to-mass ratio is set to its nominal initial condition error level and nominal random walk noise. GPS updates of the space sensor are turned on to their nominal value in this scenario, updating the space sensor position and velocity every 10 minutes. The low circular space sensor orbit is used for this analysis and the space sensor accuracy is set to its most accurate level. Ground station measurement noise is turned on. Fig. 7.4 shows that the EKF is capable of estimating the area-to-mass ratio of the RSO and, with each measurement taken, the estimation accuracy is improved. For the space sensor, Fig 7.5, the decrease in uncertainty is not as noticeable, however it can be seen that the uncertainty did not continue to grow 
until the end of the simulation. The measurements and GPS updates did have an effect on the estimation accuracy of the space sensor area-to-mass ratio. The EKF results fall within the Monte Carlo confidence intervals.

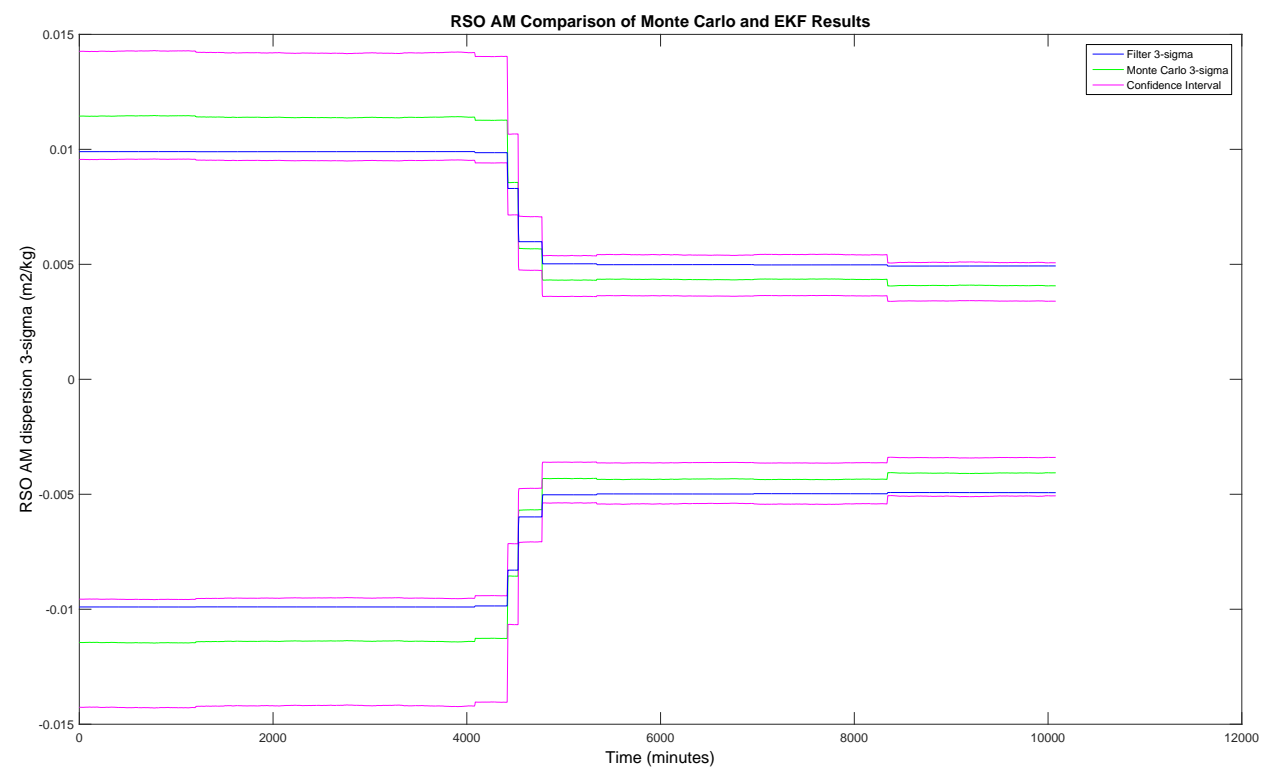

Fig. 7.4: RSO Area-to-Mass Ratio, EKF Validation, Estimation Errors 


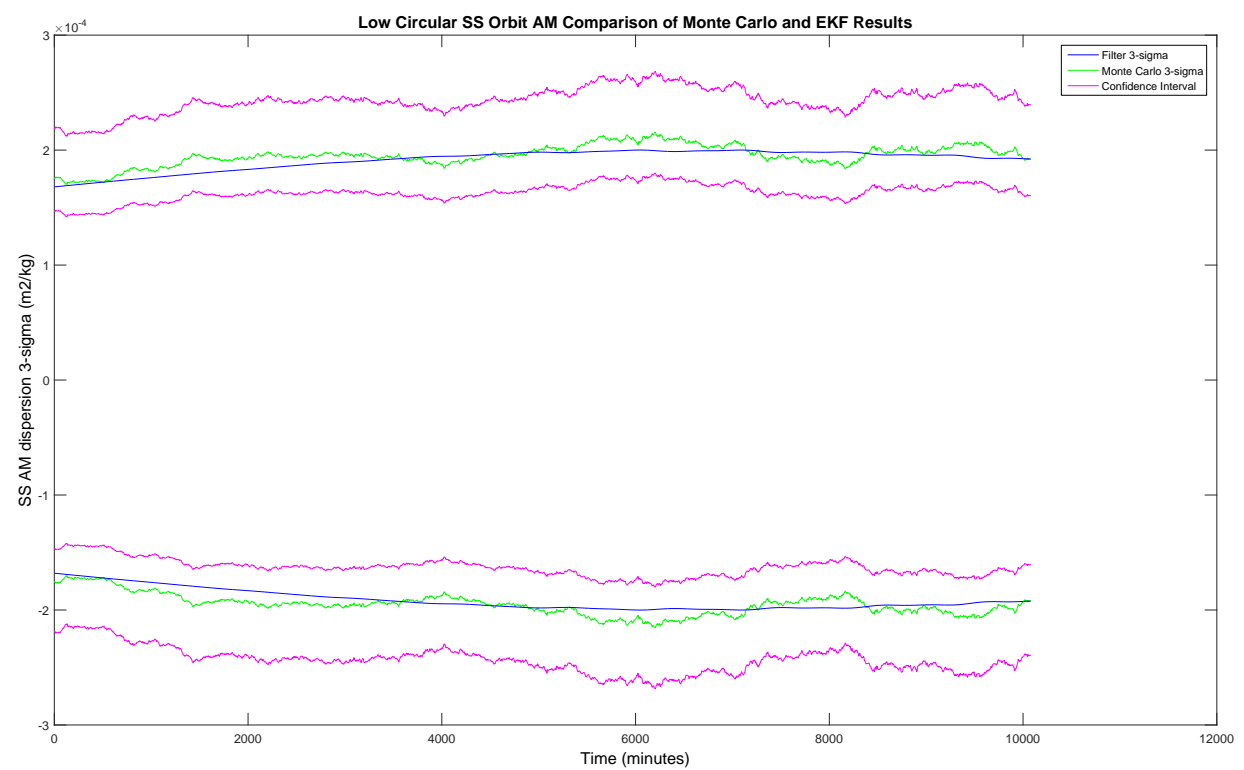

Fig. 7.5: Space Sensor Area-to-Mass Ratio, EKF Validation, Estimation Errors

\subsection{Disturbance Acceleration Estimation}

This analysis investigates the ability of the EKF to estimate the disturbance acceleration terms on the RSO and SS. The RSO disturbance acceleration term and each space sensor disturbance acceleration term is analyzed.

The RSO disturbance acceleration is shown in Fig 7.6. For this analysis, the ground station measurements are set to their nominal level. The RSO disturbance acceleration remains fairly constant throughout the simulation, therefore the measurements aren't effective at reducing the uncertainty. The EKF results fall within the Monte Carlo confidence intervals. 

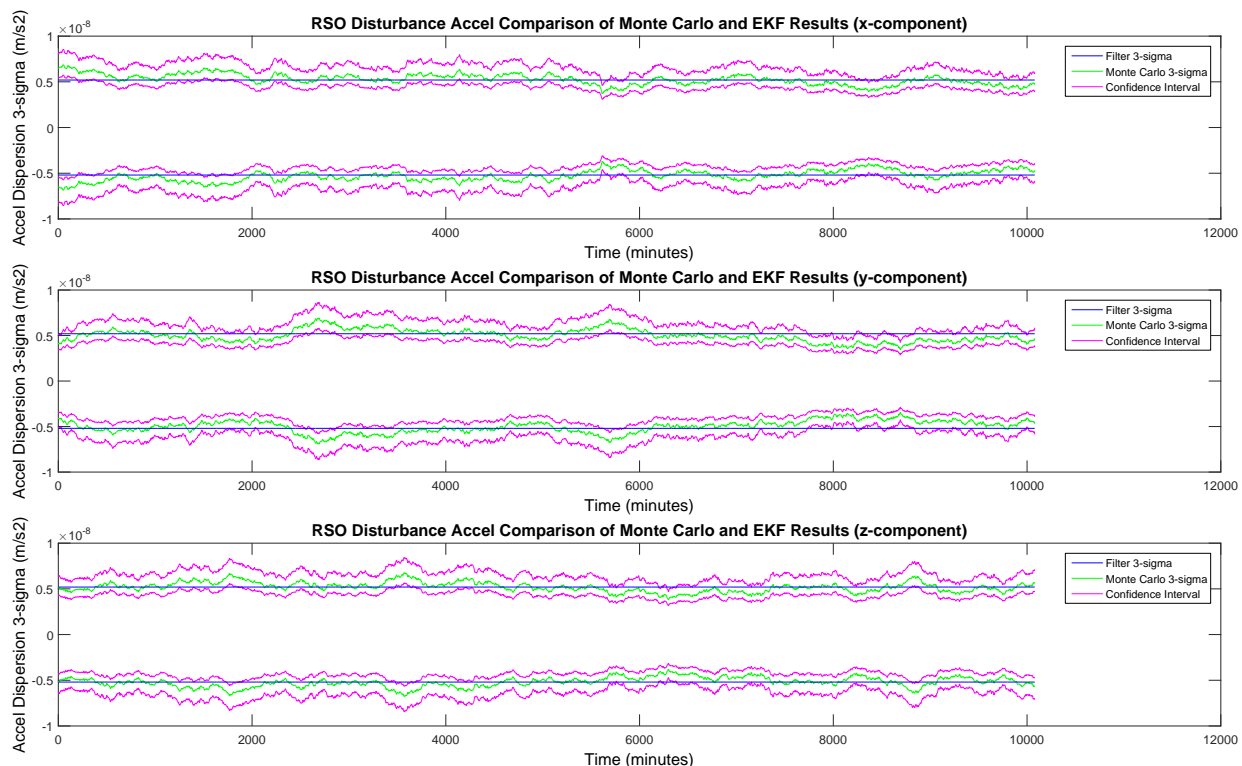

Fig. 7.6: RSO Disturbance Acceleration, EKF Validation, Estimation Errors

The space sensor low circular orbit disturbance acceleration is shown in Fig 7.7. For this analysis, the space sensor measurement accuracy and the ground station measurement accuracy are set to the nominal level. The low circular disturbance acceleration remains fairly constant throughout the simulation, therefore the measurements aren't effective at reducing the uncertainty. The EKF results fall within the Monte Carlo confidence intervals. The results for the space sensor high circular orbit and the space sensor elliptical orbit were similar and all results fall within the Monte Carlo confidence intervals. 

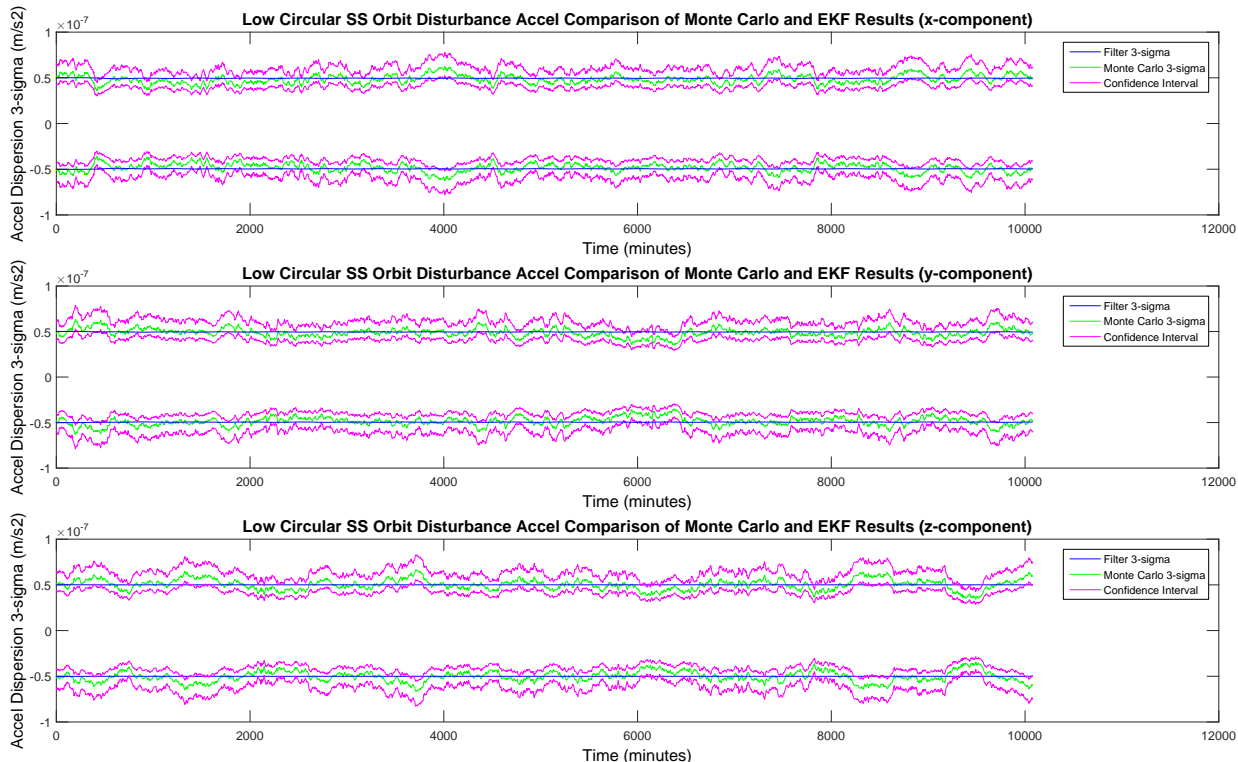

Fig. 7.7: Low Circular Space Sensor Orbit Disturbance Acceleration, EKF Validation, Estimation Errors

\subsection{Ground Station Only Measurement Validation}

The following validation runs incorporate only ground station measurements of the RSO at the nominal and high error source levels. Both the truth model and the EKF incorporate a $2 \times 2$ gravity model.

Figures $7.8-7.12$ are results from a 50 sample analysis of the RSO with only ground station measurements. All error sources and measurement uncertainties are set to the nominal level. EKF results are shown to stay within the Monte Carlo confidence intervals for all results, other than the occasional outliers. The Monte Carlo/EKF results for the Ground Station 2 bias were nearly identical to Ground Station 1 results. 

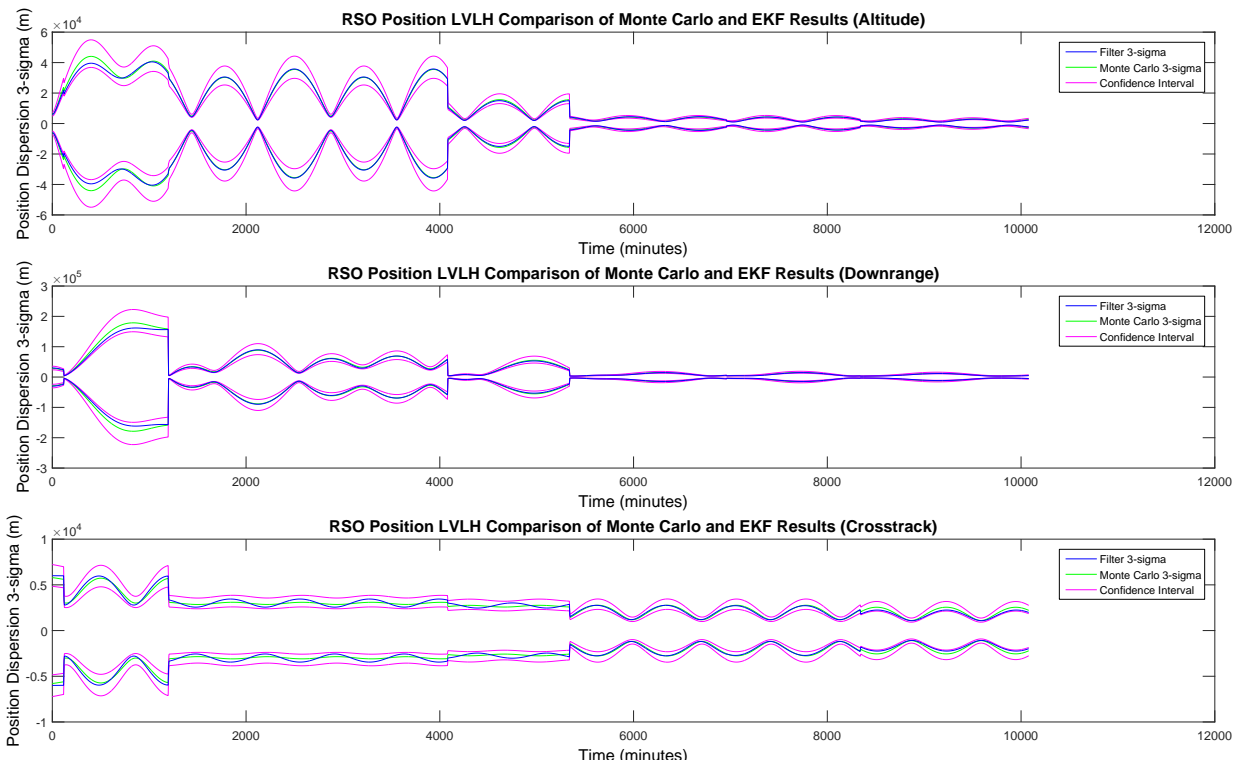

Fig. 7.8: Nominal Initial Errors - Ground Station Only Measurements, EKF Validation, RSO LVLH Position Estimation Errors
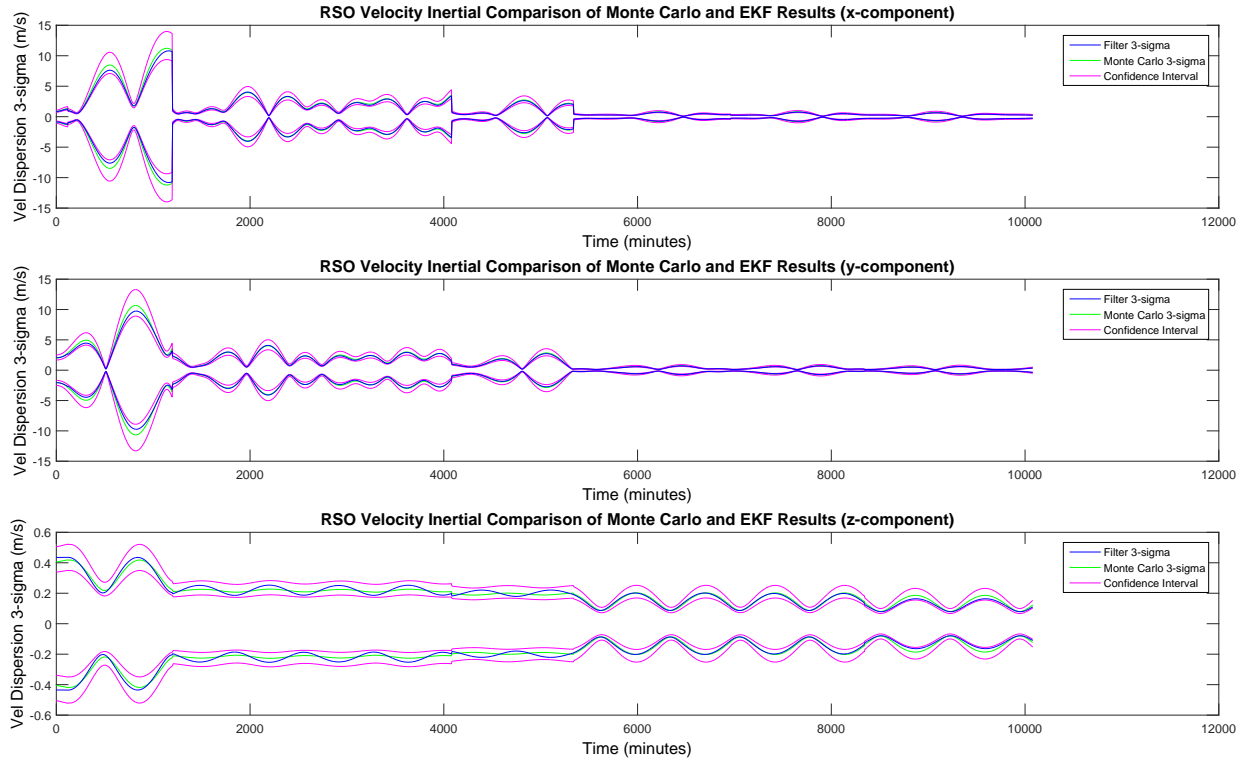

Fig. 7.9: Nominal Initial Errors - Ground Station Only Measurements, EKF Validation, RSO Velocity Estimation Errors 


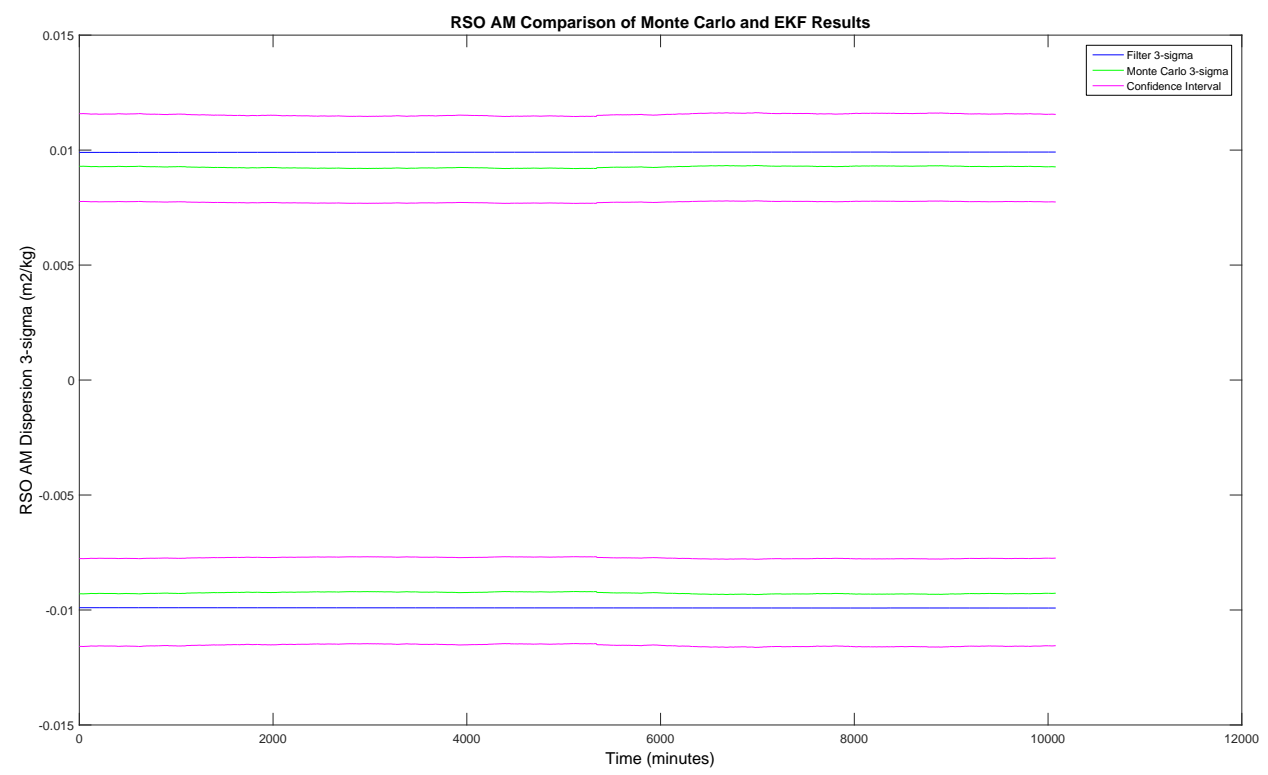

Fig. 7.10: Nominal Initial Errors - Ground Station Only Measurements, EKF Validation, RSO Area-to-Mass Ratio Estimation Error
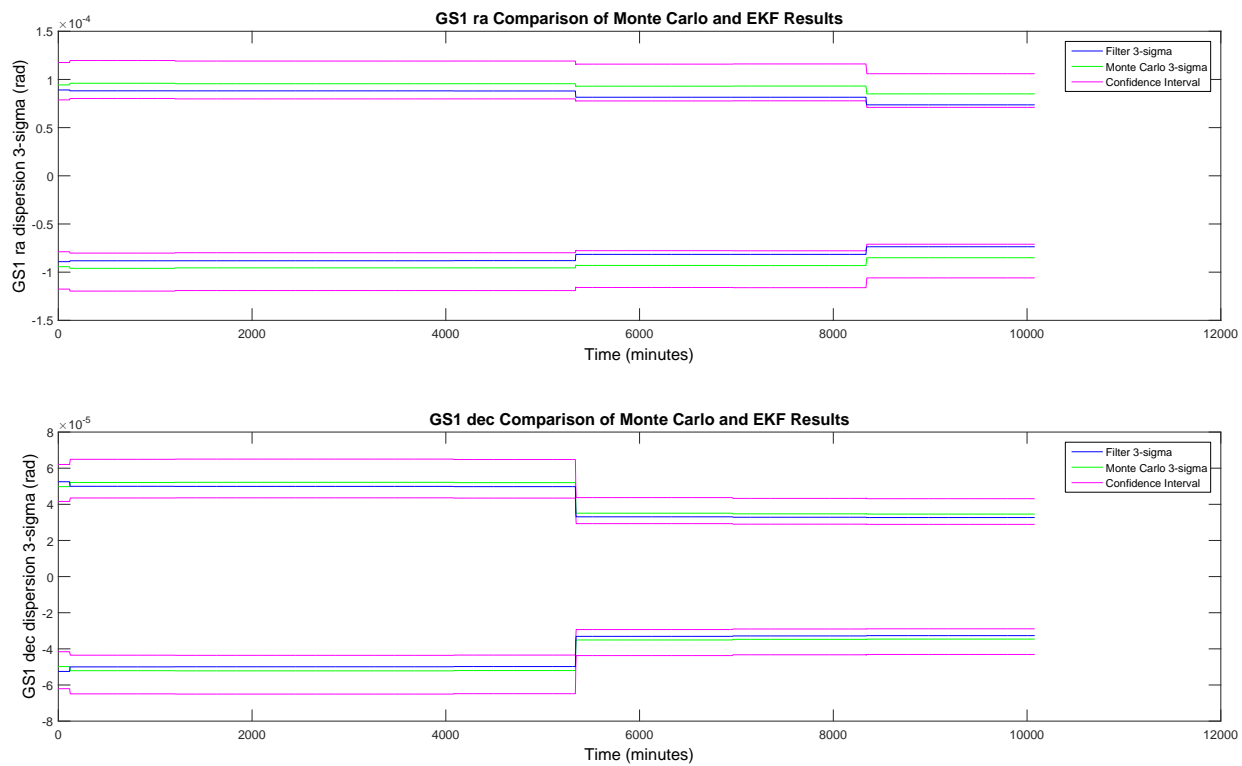

Fig. 7.11: Nominal Initial Errors - Ground Station Only Measurements, EKF Validation, Ground Station 1 Biases Estimation Errors 

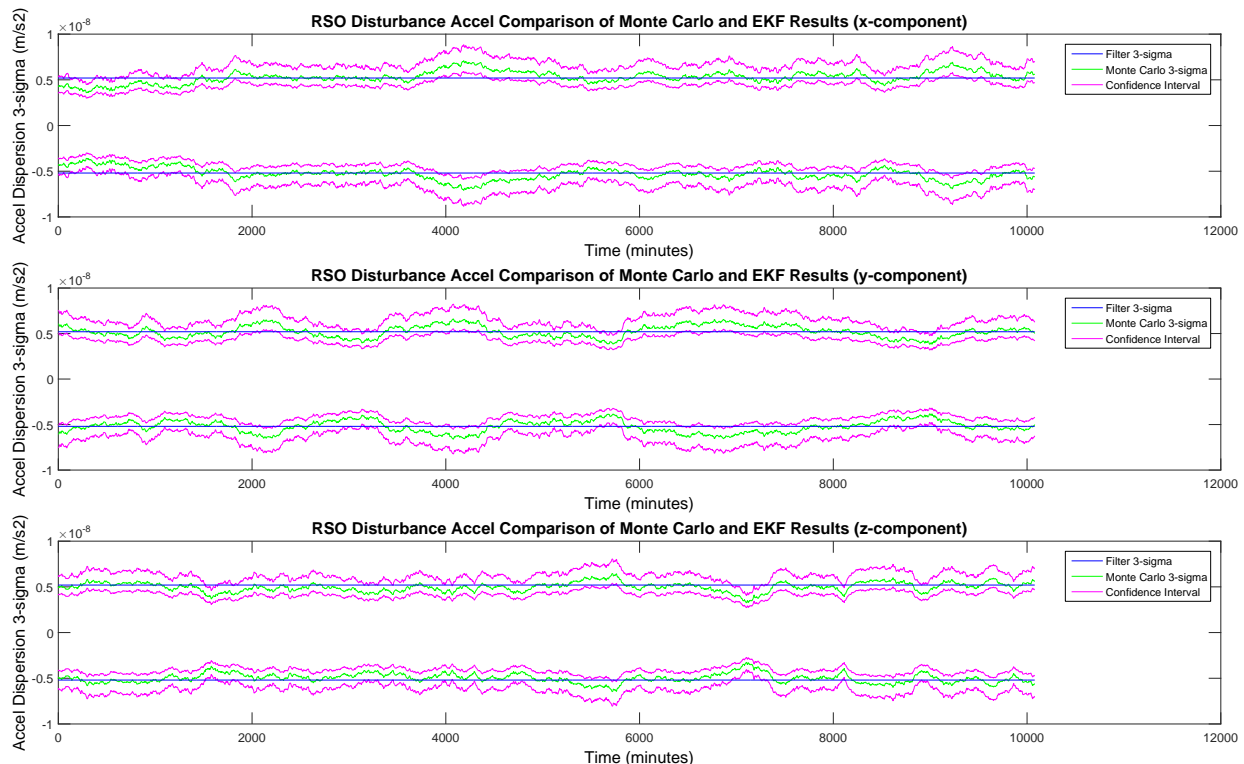

Fig. 7.12: Nominal Initial Errors - Ground Station Only Measurements, EKF Validation, RSO Disturbance Accels Estimation Errors

Although the magnitudes of the Monte Carlo/EKF results are larger than the nominal error cases, all results for the high level error case have similar characteristics as the low error case. The one exception is the area-to-mass ratio shown in Figure 7.13. 


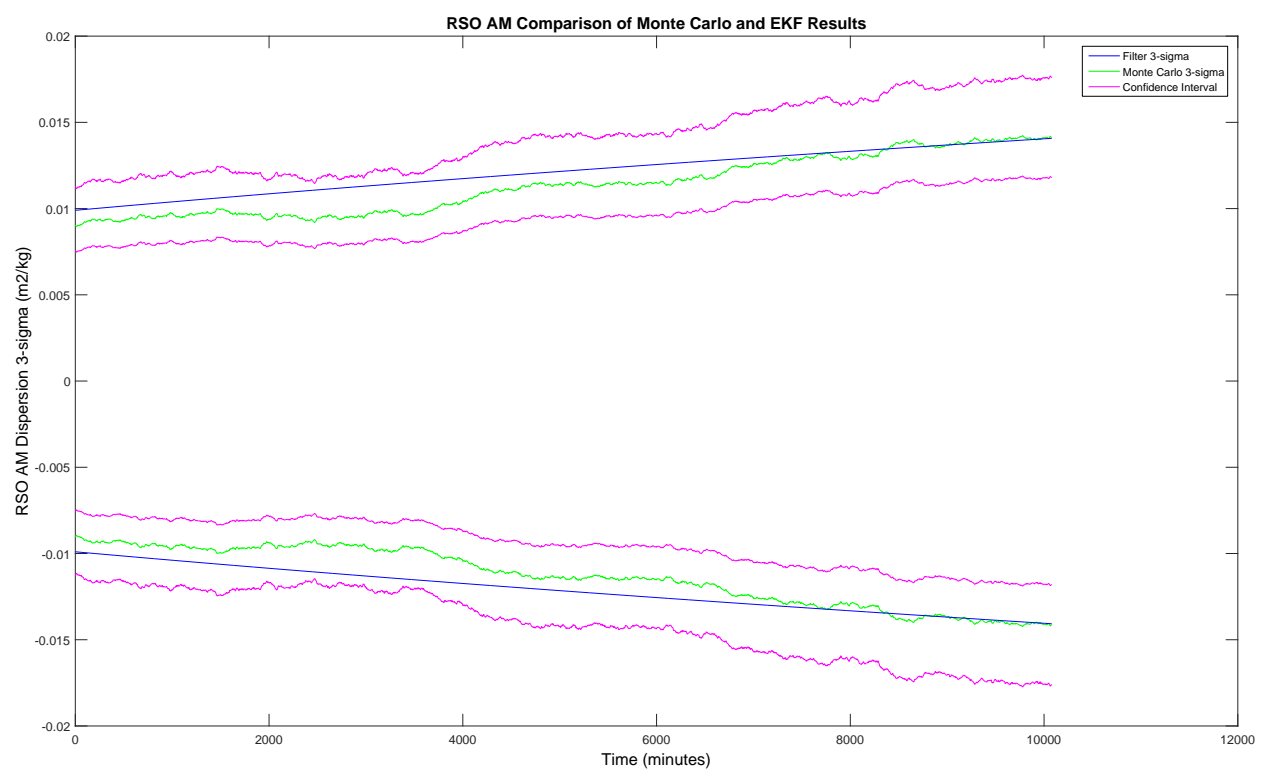

Fig. 7.13: High Initial Errors - Ground Station Only Measurements, EKF Validation, RSO Area-to-Mass Ratio Estimation Error

\subsection{Space Sensor and Ground Station Measurement Validation}

The following validation runs incorporate both space sensor and ground station measurements of the RSO. Each space sensor orbit: low circular, high circular, and elliptical, is analyzed for nominal and high error source levels. The space sensor position and velocity is updated through the use of GPS. Both the truth model and the EKF incorporate a $2 \times 2$ gravity model. All relevant plots are shown for the low circular nominal error source analysis. The remaining analysis results will only show plots for the research critical results, the RSO LVLH position. All results met the condition of the EKF results being within confidence intervals.

\subsubsection{Low Circular Space Sensor Orbit}

Figures 7.14 - 7.24 are results from a 50 sample analysis of the RSO with low circular orbit space sensor and ground station measurements. All error sources are set to the nominal level. EKF results are shown to stay within the Monte Carlo confidence intervals for all 
results, other than the occasional outliers.
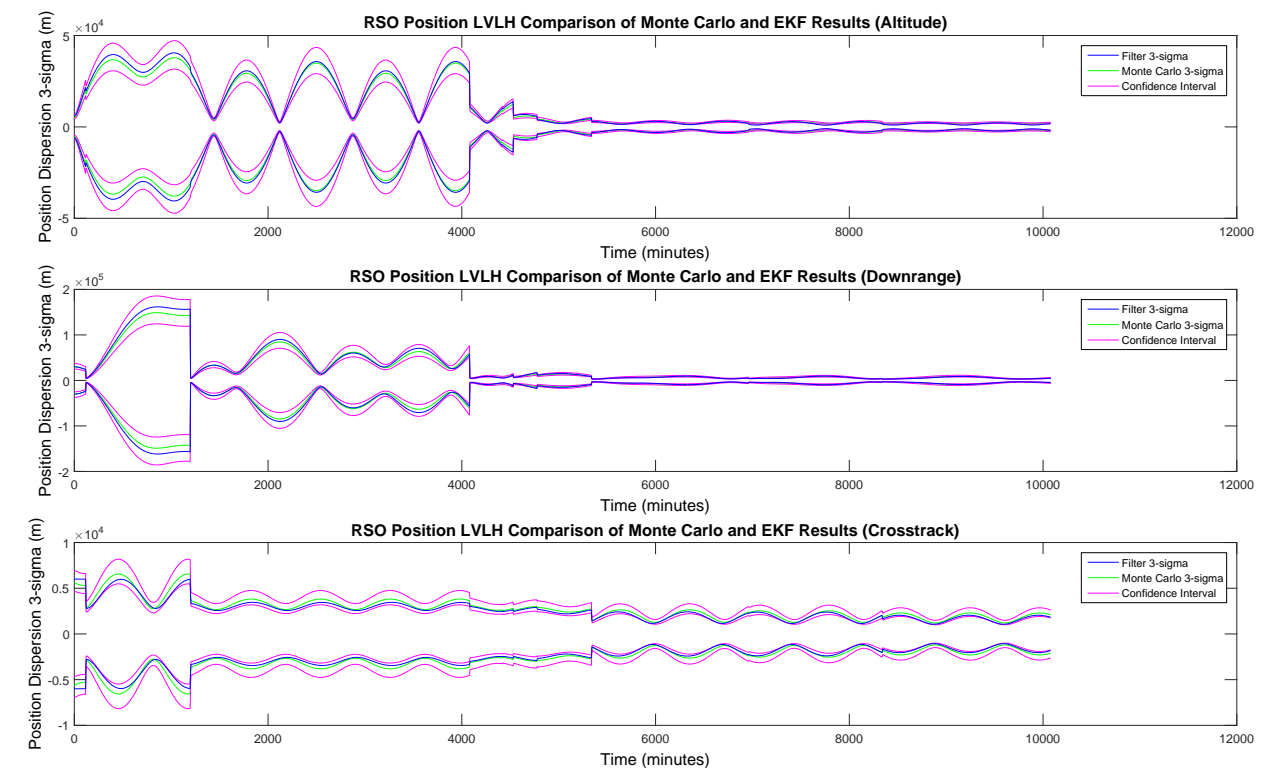

Fig. 7.14: Nominal Initial Errors - Ground Station and Low Circular SS Measurements, EKF Validation, RSO LVLH Position Estimation Errors 

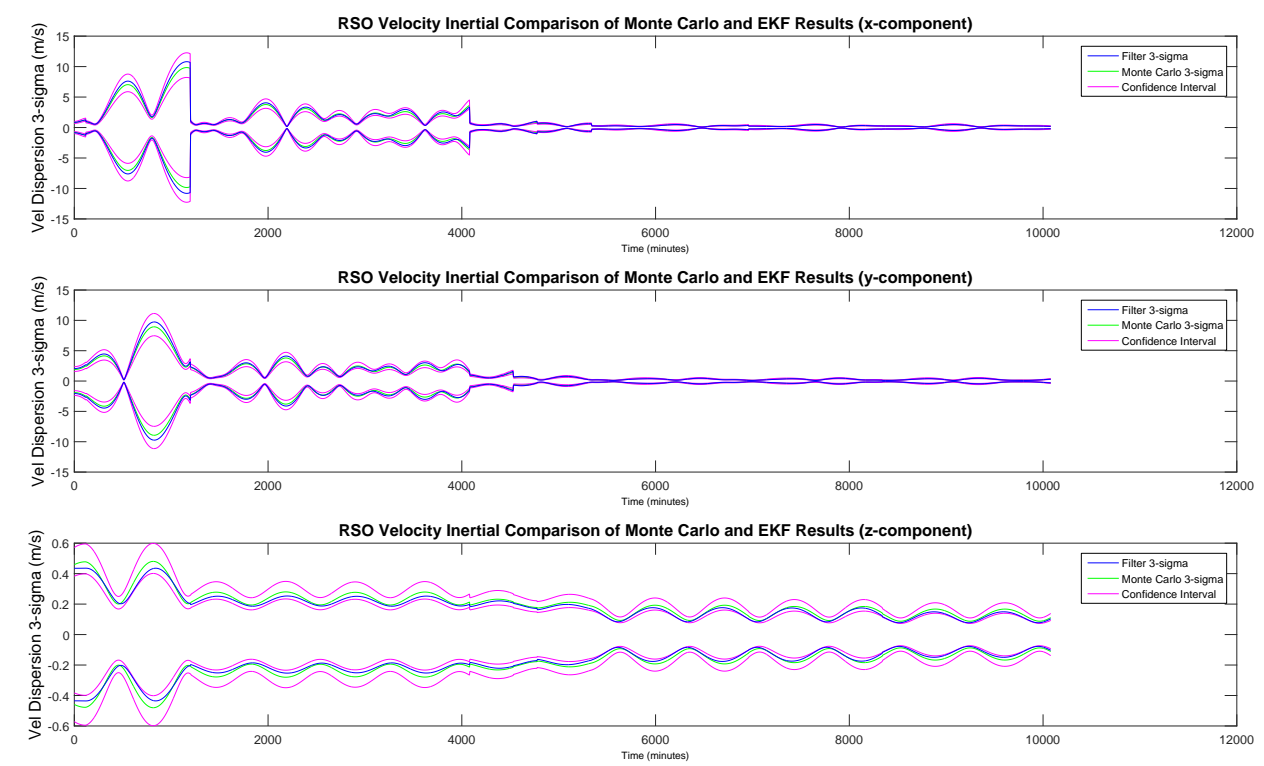

Fig. 7.15: Nominal Initial Errors - Ground Station and Low Circular SS Measurements, EKF Validation, RSO Inertial Velocity Estimation Errors

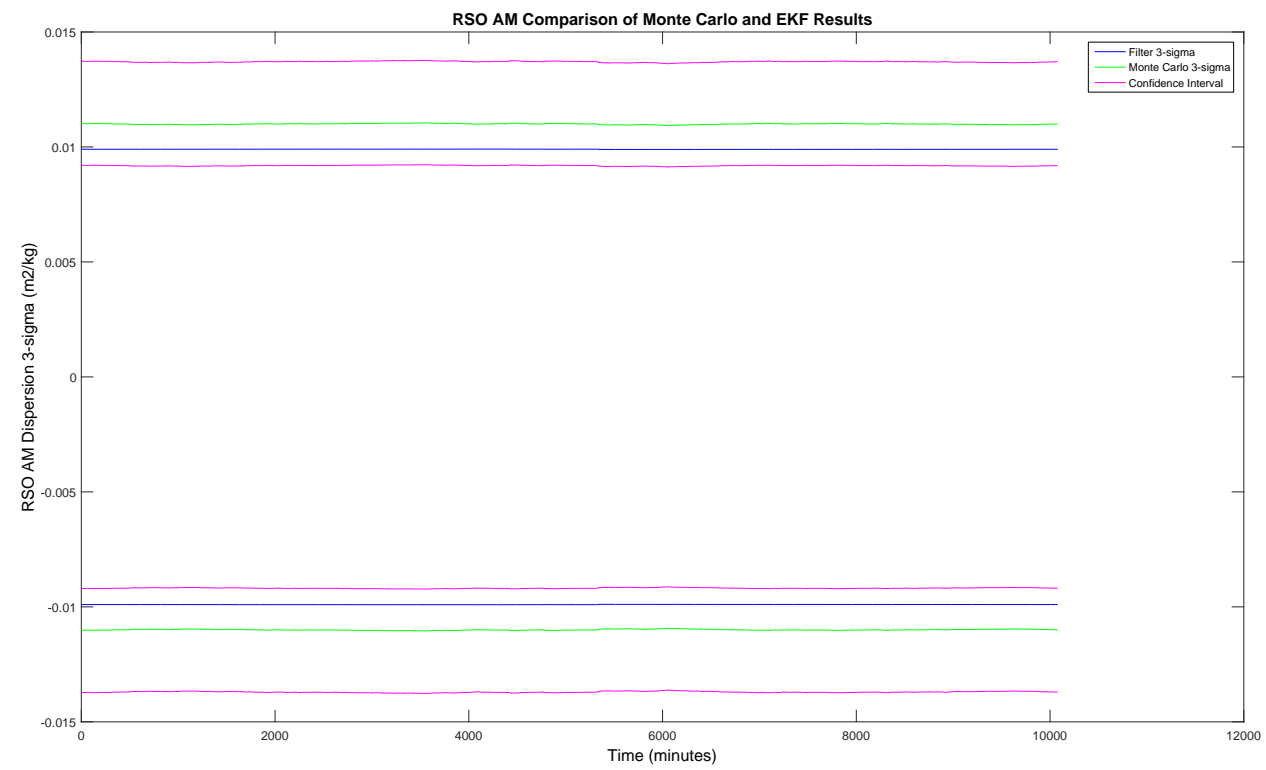

Fig. 7.16: Nominal Initial Errors - Ground Station and Low Circular SS Measurements, EKF Validation, RSO Area-to-Mass Ratio Estimation Error 

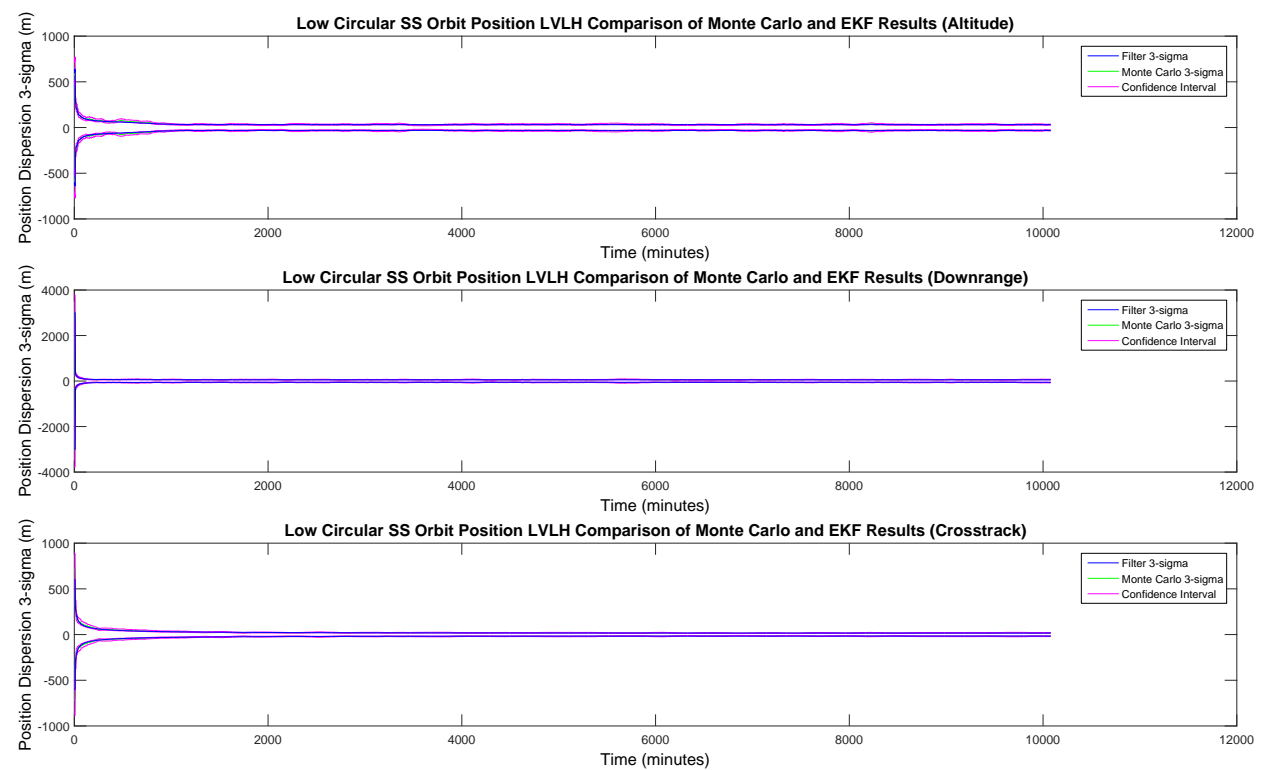

Fig. 7.17: Nominal Initial Errors - Ground Station and Low Circular SS Measurements, EKF Validation, SS LVLH Position Estimation Errors
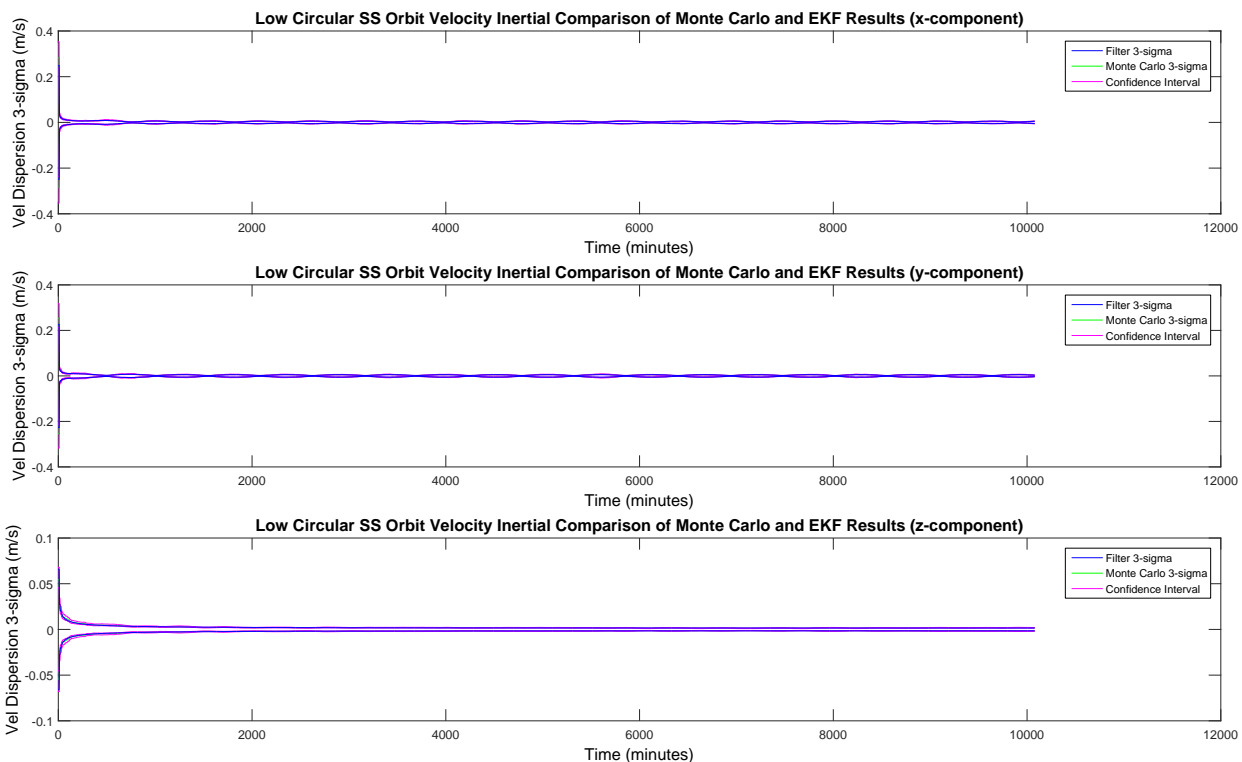

Fig. 7.18: Nominal Initial Errors - Ground Station and Low Circular SS Measurements, EKF Validation, SS Inertial Velocity Estimation Errors 


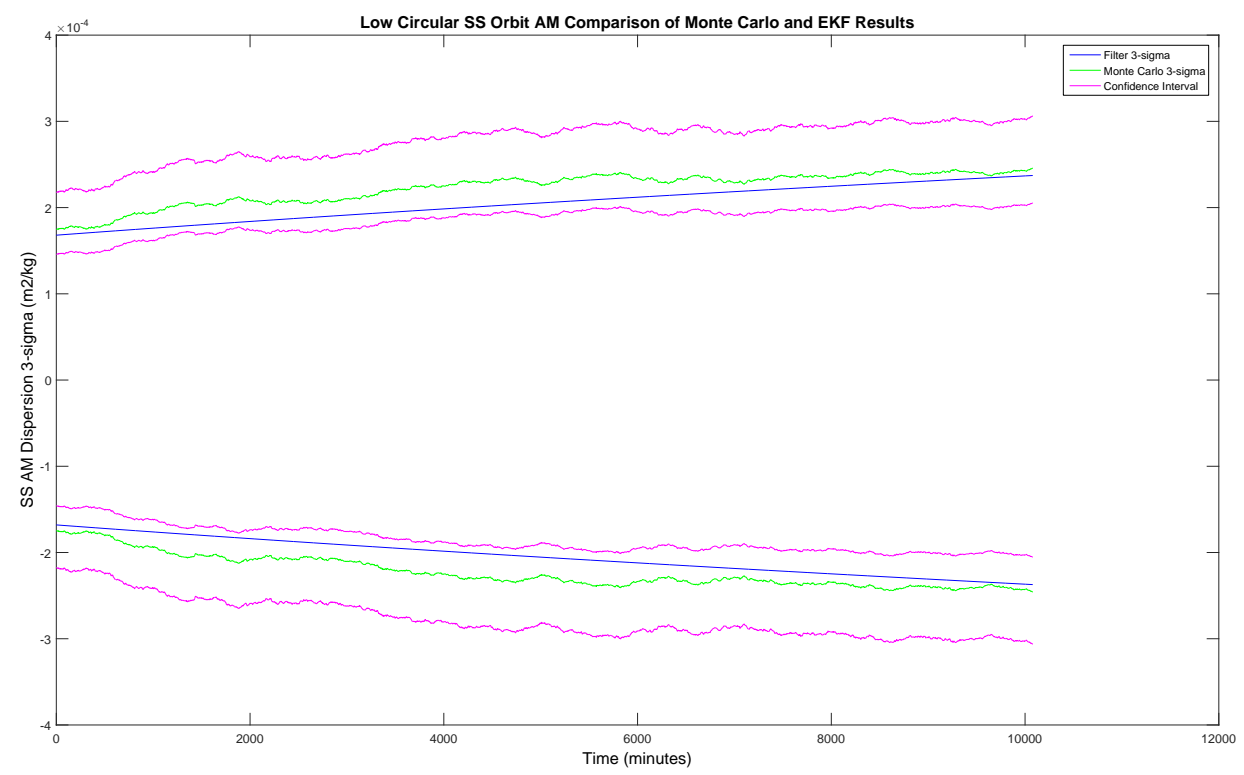

Fig. 7.19: Nominal Initial Errors - Ground Station and Low Circular SS Measurements, EKF Validation, SS Area-to-Mass Ratio Estimation Error
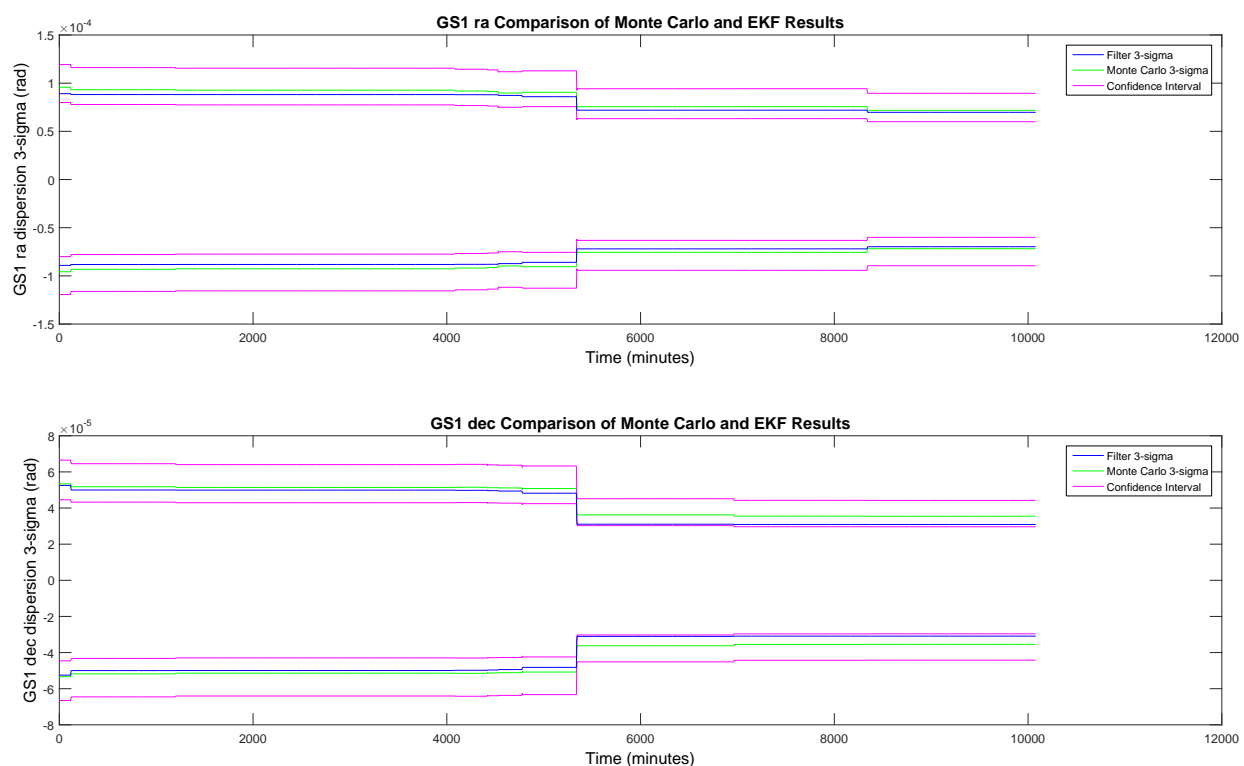

Fig. 7.20: Nominal Initial Errors - Ground Station and Low Circular SS Measurements, EKF Validation, Ground Station 1 Biases Estimation Errors 

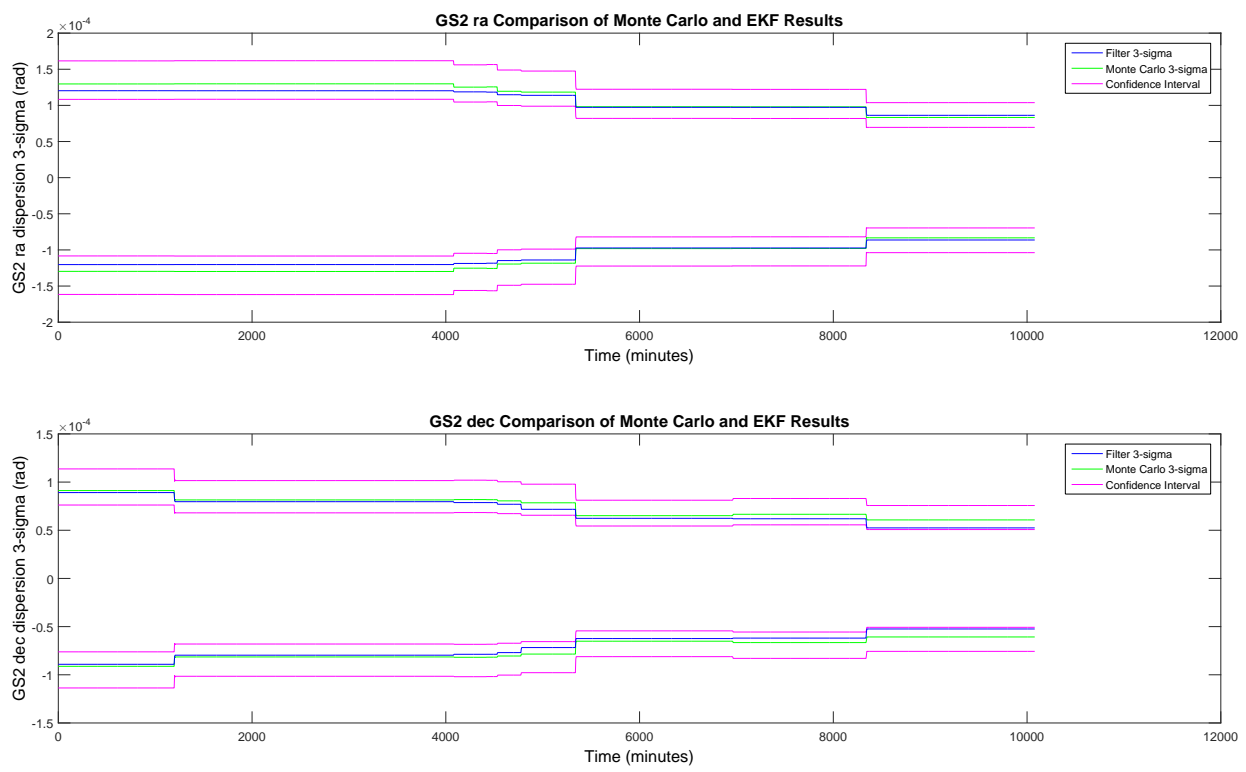

Fig. 7.21: Nominal Initial Errors - Ground Station and Low Circular SS Measurements, EKF Validation, Ground Station 2 Biases Estimation Errors
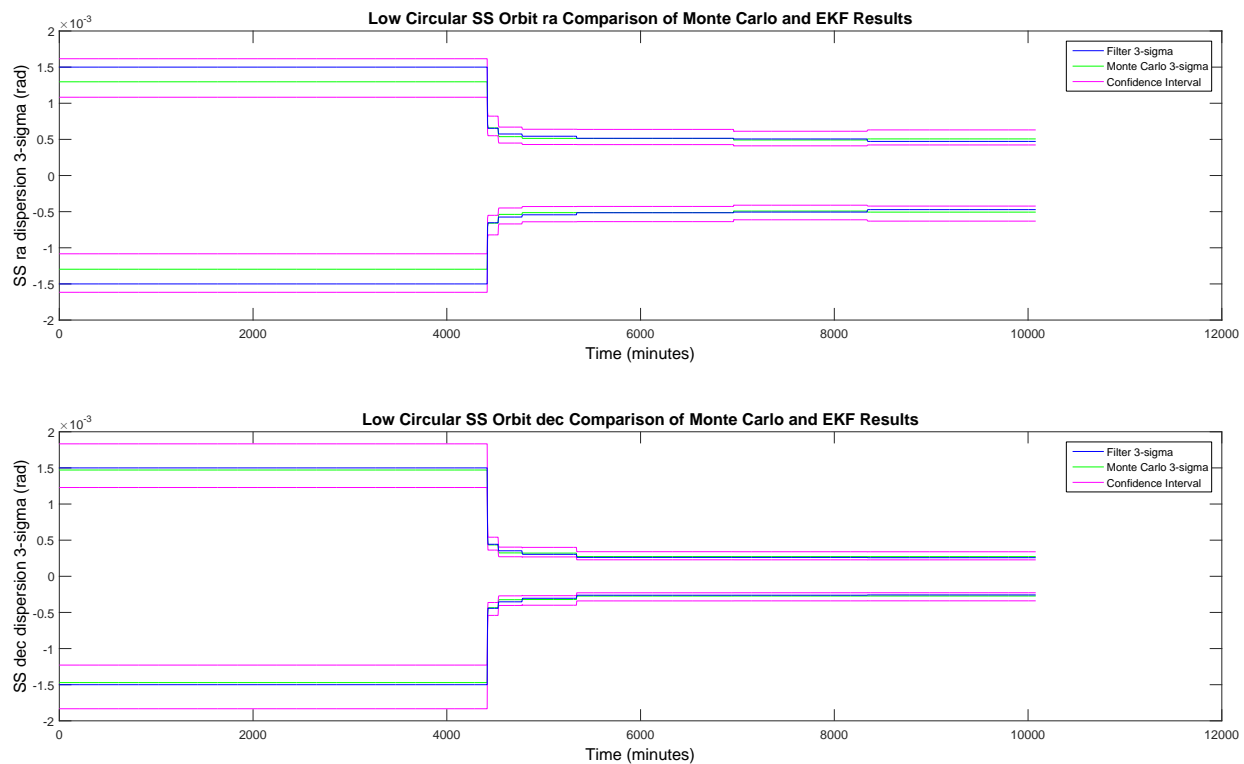

Fig. 7.22: Nominal Initial Errors - Ground Station and Low Circular SS Measurements, EKF Validation, SS Biases Estimation Errors 

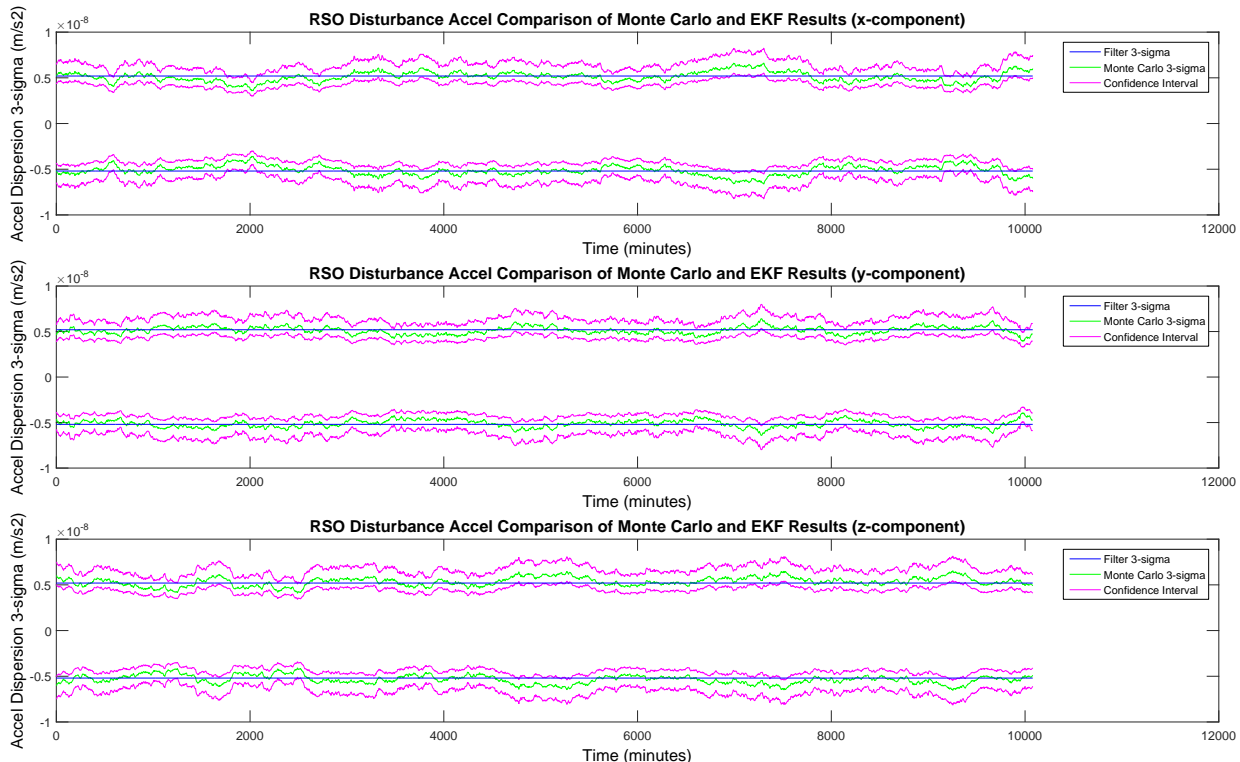

Fig. 7.23: Nominal Initial Errors - Ground Station and Low Circular SS Measurements, EKF Validation, RSO Disturbance Accels Estimation Errors
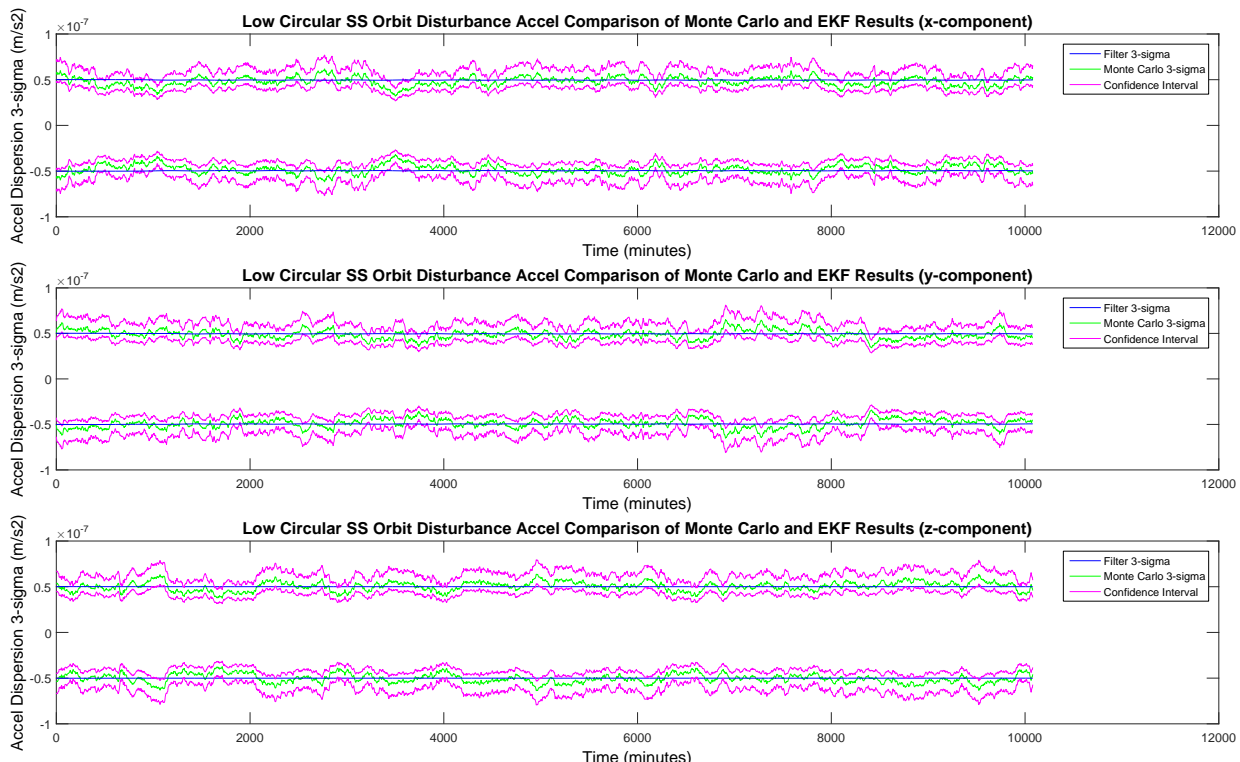

Fig. 7.24: Nominal Initial Errors - Ground Station and Low Circular SS Measurements, EKF Validation, SS Disturbance Accels Estimation Errors 
Figure 7.25 shows results from a 50 sample analysis of the RSO with low circular orbit space sensor and ground station measurements. All error sources are set to the high level. EKF results are shown to stay within the Monte Carlo confidence intervals for all results, other than the occasional outliers.
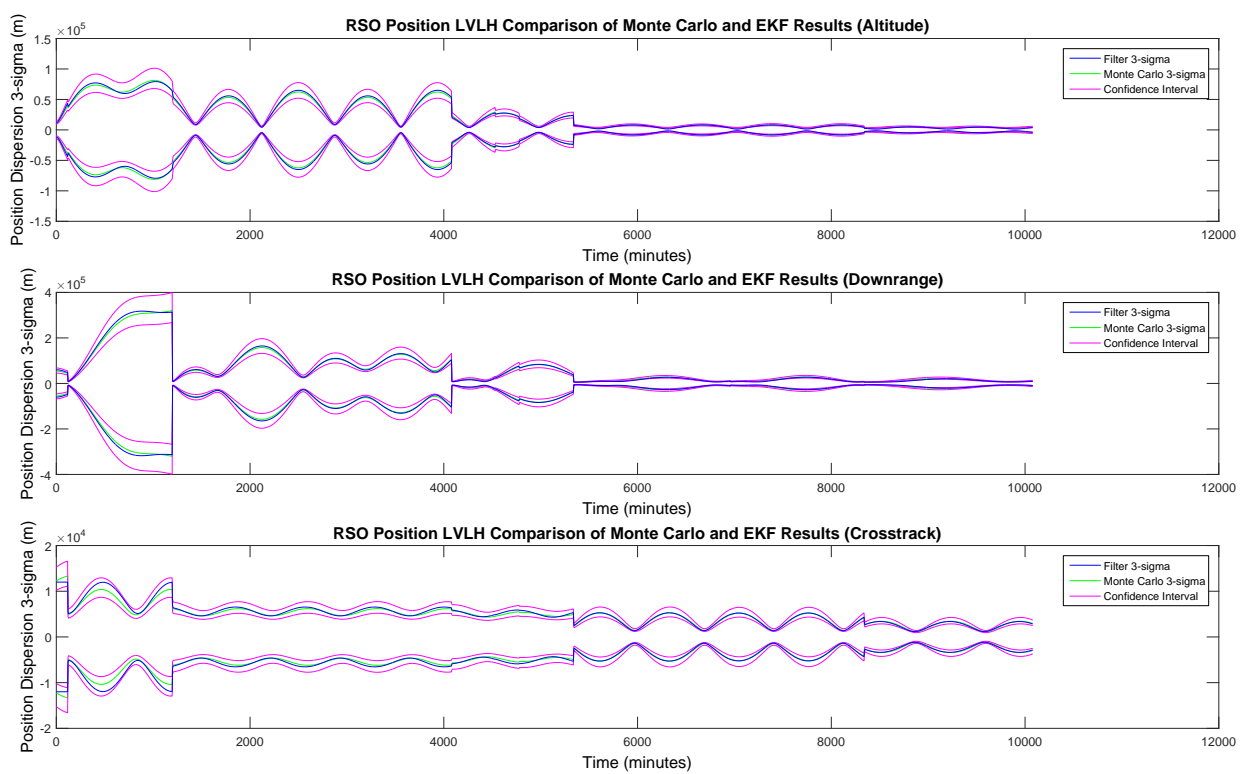

Fig. 7.25: High Initial Errors - Ground Station and Low Circular SS Measurements, EKF Validation, RSO LVLH Position Estimation Errors

\subsubsection{High Circular Space Sensor Orbit}

Figure 7.26 shows results from a 50 sample analysis of the RSO with high circular orbit space sensor and ground station measurements. All error sources are set to the nominal level. EKF results are shown to stay within the Monte Carlo confidence intervals for all results, other than the occasional outliers. 

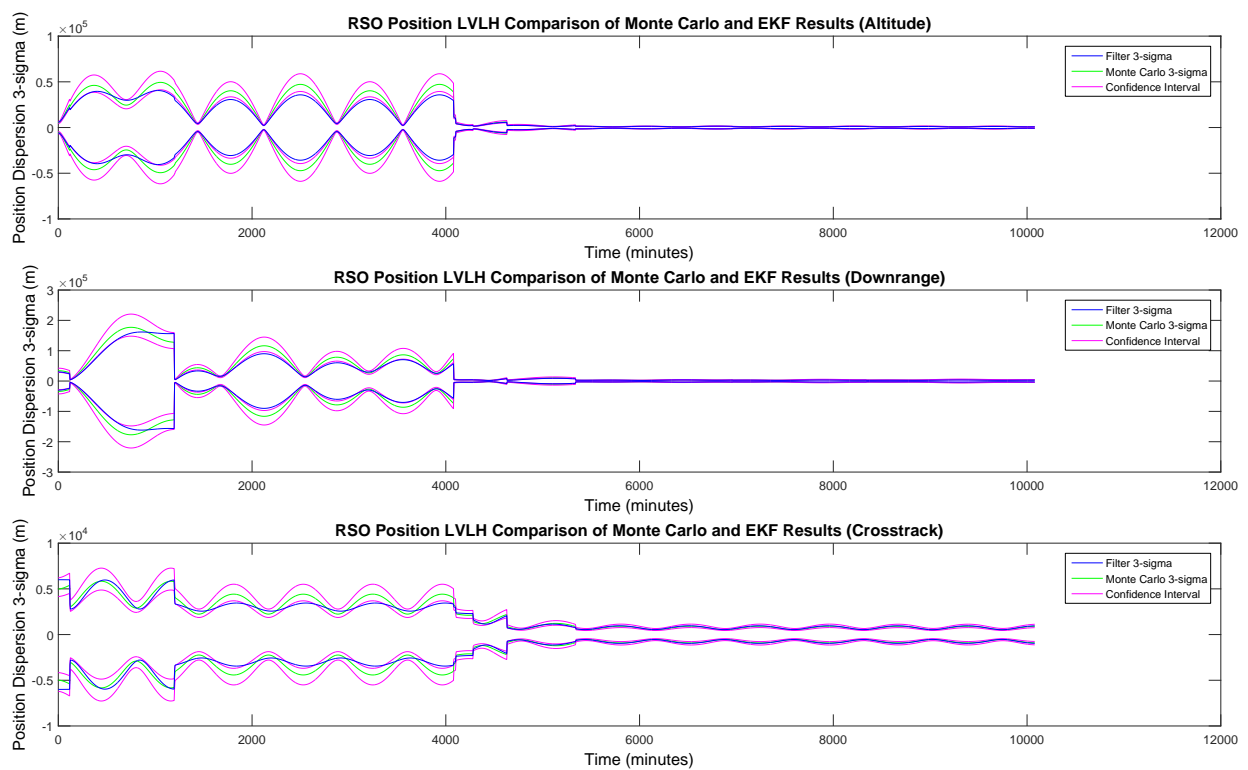

Fig. 7.26: Nominal Initial Errors - Ground Station and High Circular SS Measurements, EKF Validation, RSO LVLH Position Estimation Errors

Figure 7.27 shows results from a 50 sample analysis of the RSO with high circular orbit space sensor and ground station measurements. All error sources are set to the high level. EKF results are shown to stay within the Monte Carlo confidence intervals for all results, other than the occasional outliers. 

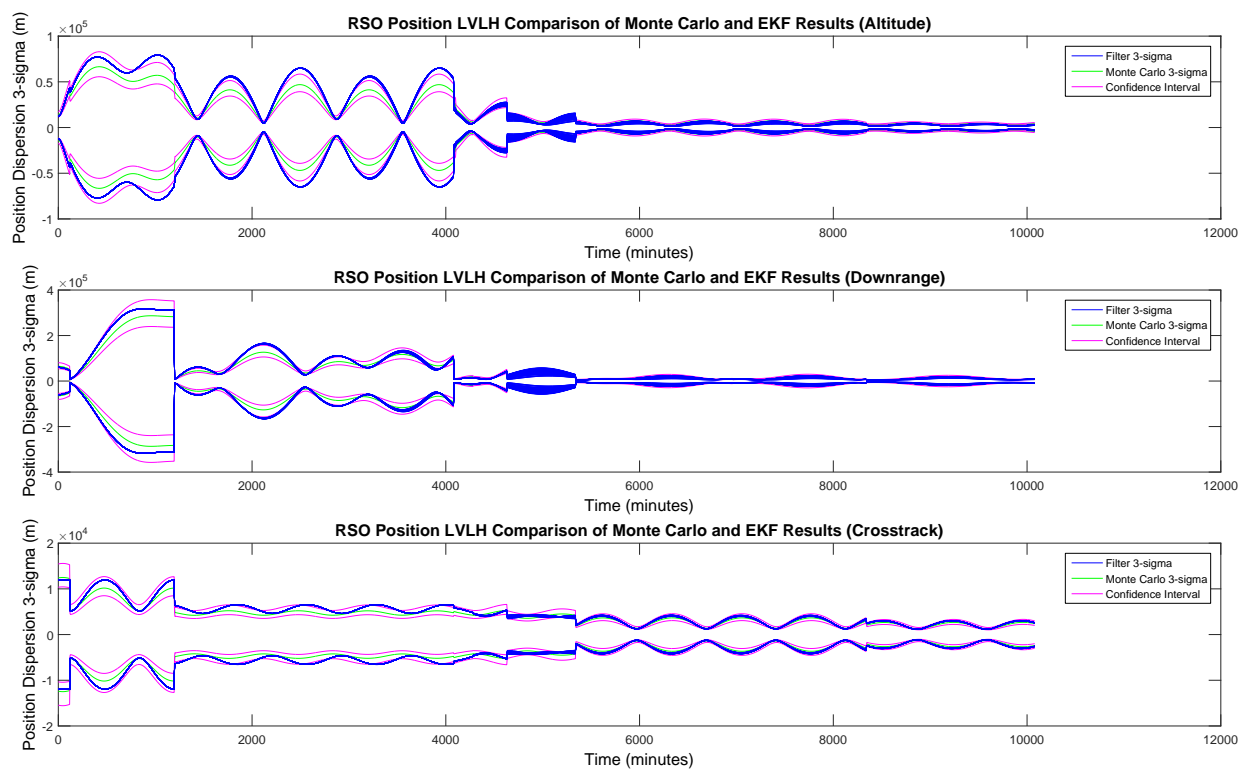

Fig. 7.27: High Initial Errors - Ground Station and High Circular SS Measurements, EKF Validation, RSO LVLH Position Estimation Errors

\subsubsection{Elliptical Space Sensor Orbit}

Figure 7.28 shows results from a 50 sample analysis of the RSO with elliptical orbit space sensor and ground station measurements. All error sources are set to the nominal level. EKF results are shown to stay within the Monte Carlo confidence intervals for all results, other than the occasional outliers. 

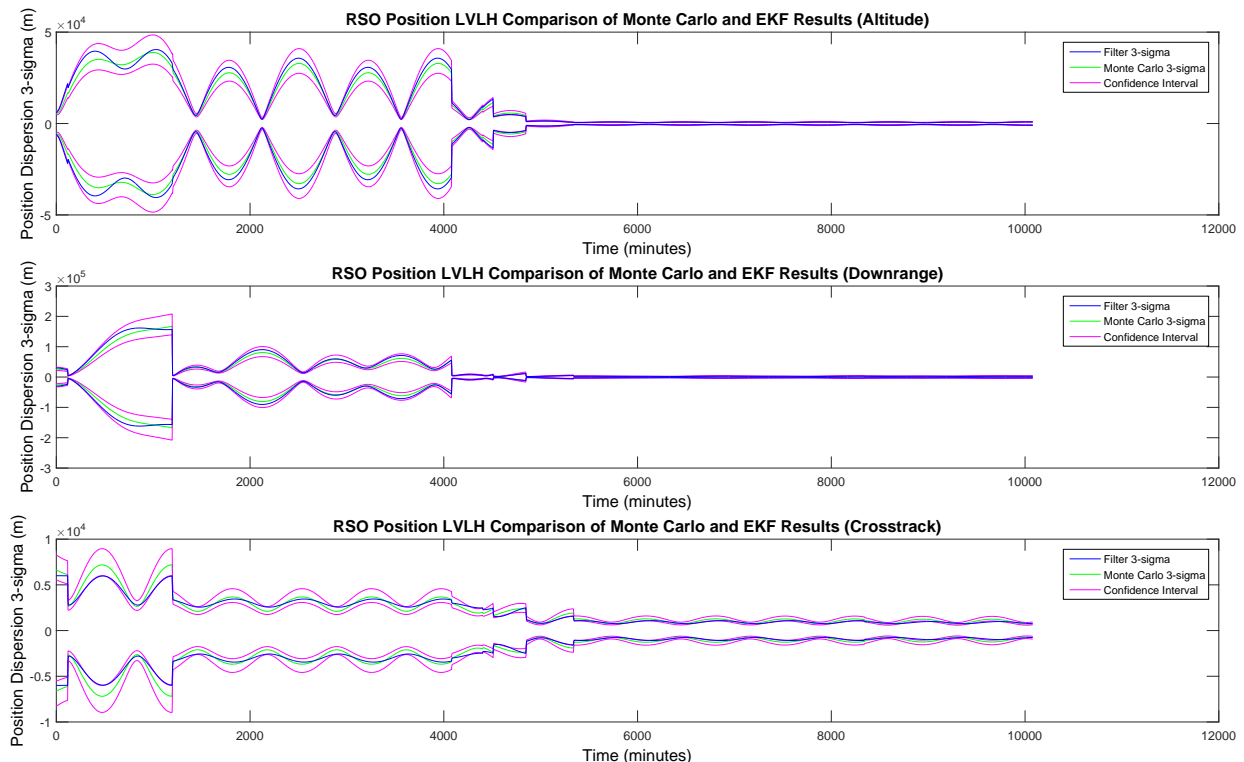

Fig. 7.28: Nominal Initial Errors - Ground Station and Elliptical SS Measurements, EKF Validation, RSO LVLH Position Estimation Errors

Figure 7.29 shows results from a 50 sample analysis of the RSO with elliptical orbit space sensor and ground station measurements. All error sources are set to the high level. EKF results are shown to stay within the Monte Carlo confidence intervals for all results, other than the occasional outliers. 

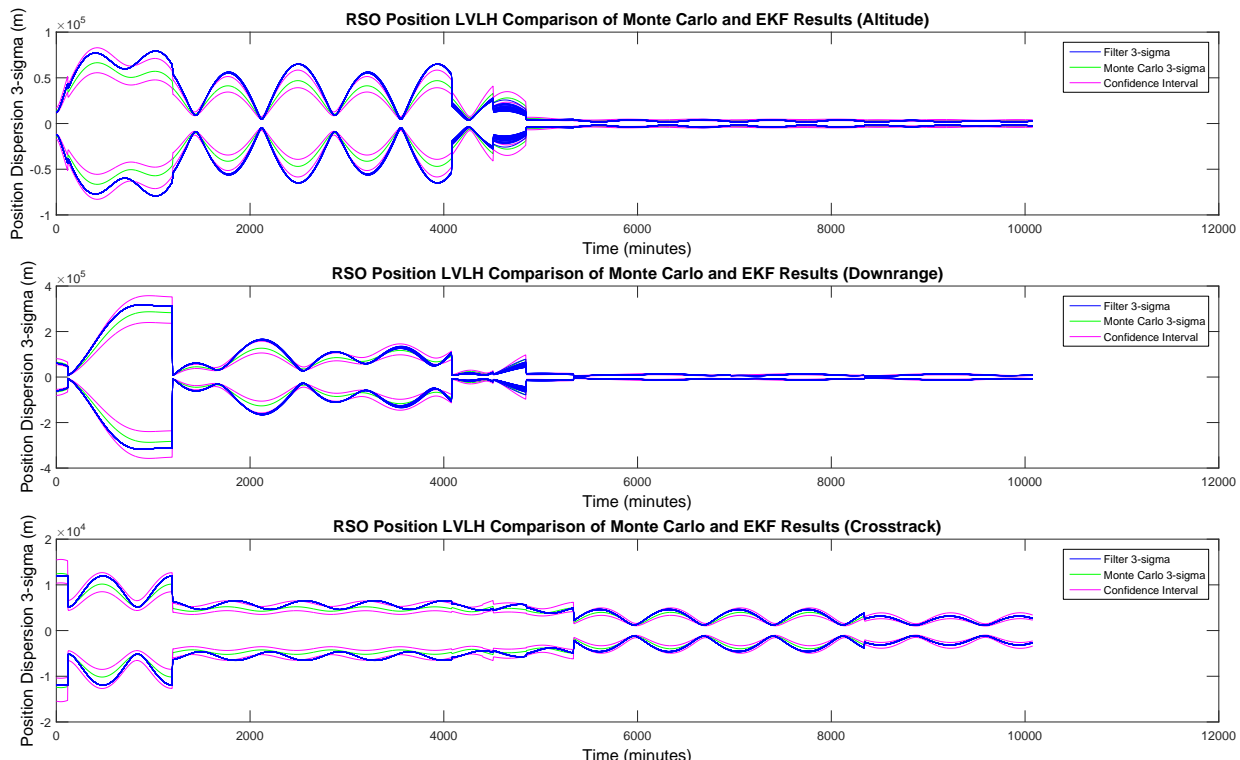

Fig. 7.29: High Initial Errors - Ground Station and Elliptical SS Measurements, EKF Validation, RSO LVLH Position Estimation Errors

\subsection{Truth Environment Validation}

The following validation runs incorporate both space sensor and ground station measurements of the RSO. Each space sensor orbit: low circular, high circular, and elliptical, is analyzed for low error source levels. The space sensor position and velocity is updated through the use of GPS. The truth model incorporates an $18 \times 18$ gravity model without disturbance accelerations and the EKF incorporates a $2 \times 2$ gravity model with disturbance accelerations. All relevant plots are shown for the ground station measurements only and ground plus low circular space sensor measurements with nominal error sources. The remaining analysis results will only show plots for the research critical results, the RSO LVLH position, as well as the space sensor disturbance acceleration.

The objective of this section is to show that the EKF performs well in an $18 \times 18$ gravity field environment by simply modeling all high-order gravity terms with a tuned first-order Markov process. To ensure the higher-order terms are not being masked by other error 
sources, all errors are set to the low level.

\subsubsection{Ground Station Only Measurements}

Figures 7.30 through 7.34 are results from a 50 sample analysis of the RSO with ground station measurements of the RSO. All error sources are set to the low level. The main difference between these results and the results from the truth model $2 \times 2$ gravity analysis is seen in the disturbance acceleration plot of the RSO. It can be seen that the EKF disturbance acceleration lies outside of the Monte Carlo confidence intervals. However, the EKF disturbance acceleration model was built to be conservative. The EKF slightly overestimates the effect of the disturbance accelerations in all scenarios. The Monte Carlo/EKF results for the Ground Station 2 bias were nearly identical to Ground Station 1 results.
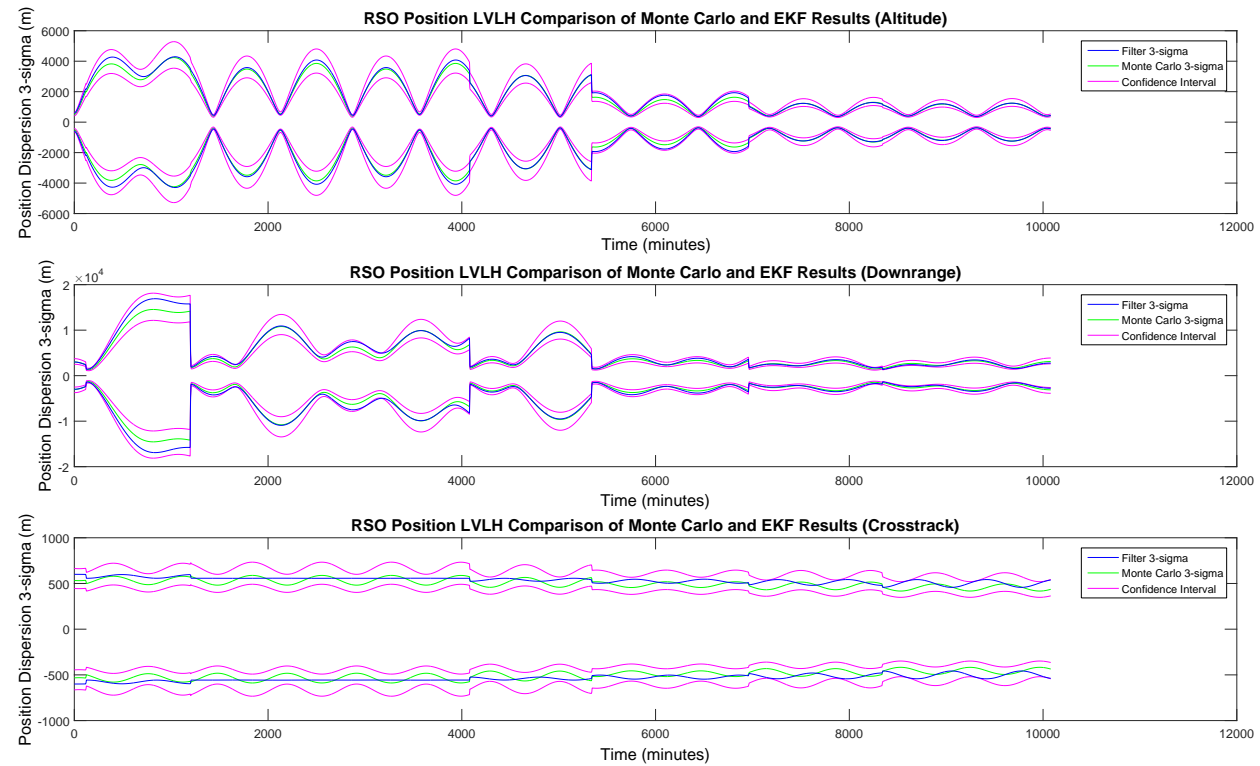

Fig. 7.30: Low Initial Errors - Ground Station Only Measurements, EKF Validation, RSO LVLH Position Estimation Errors 

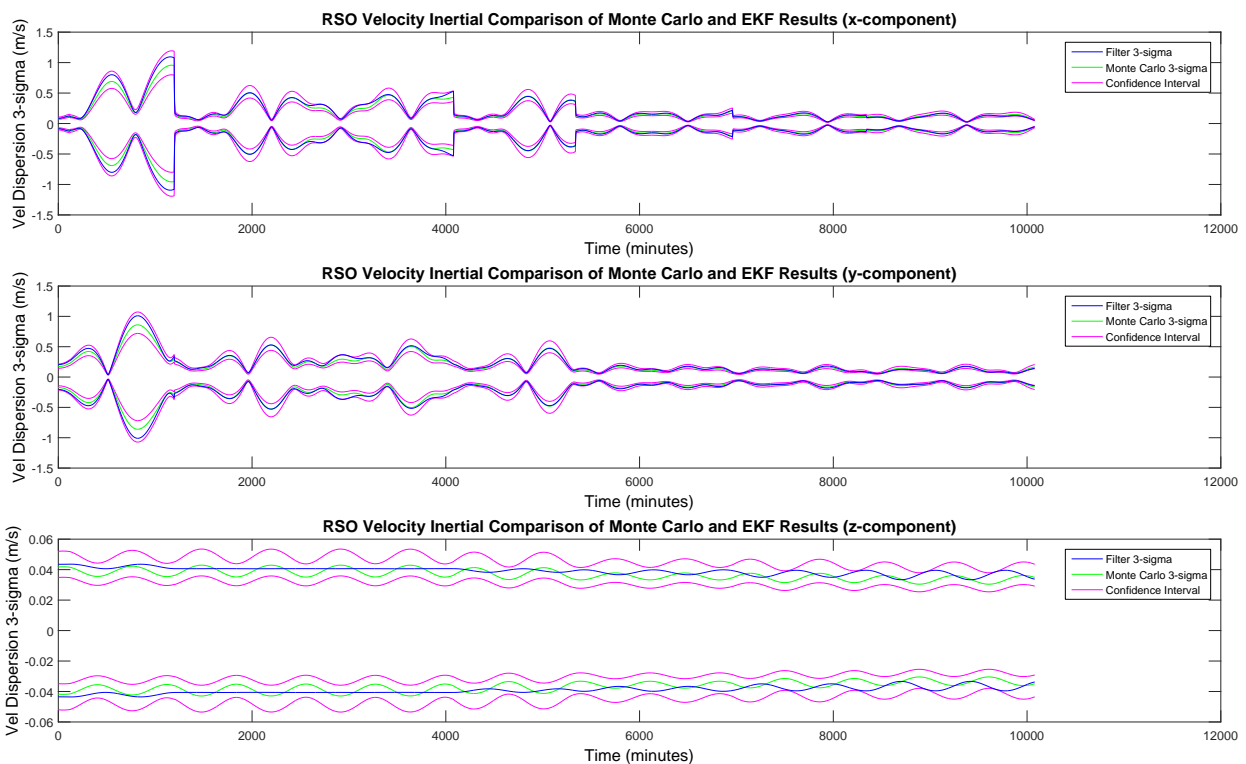

Fig. 7.31: Low Initial Errors - Ground Station Only Measurements, EKF Validation, RSO Inertial Velocity Estimation Errors

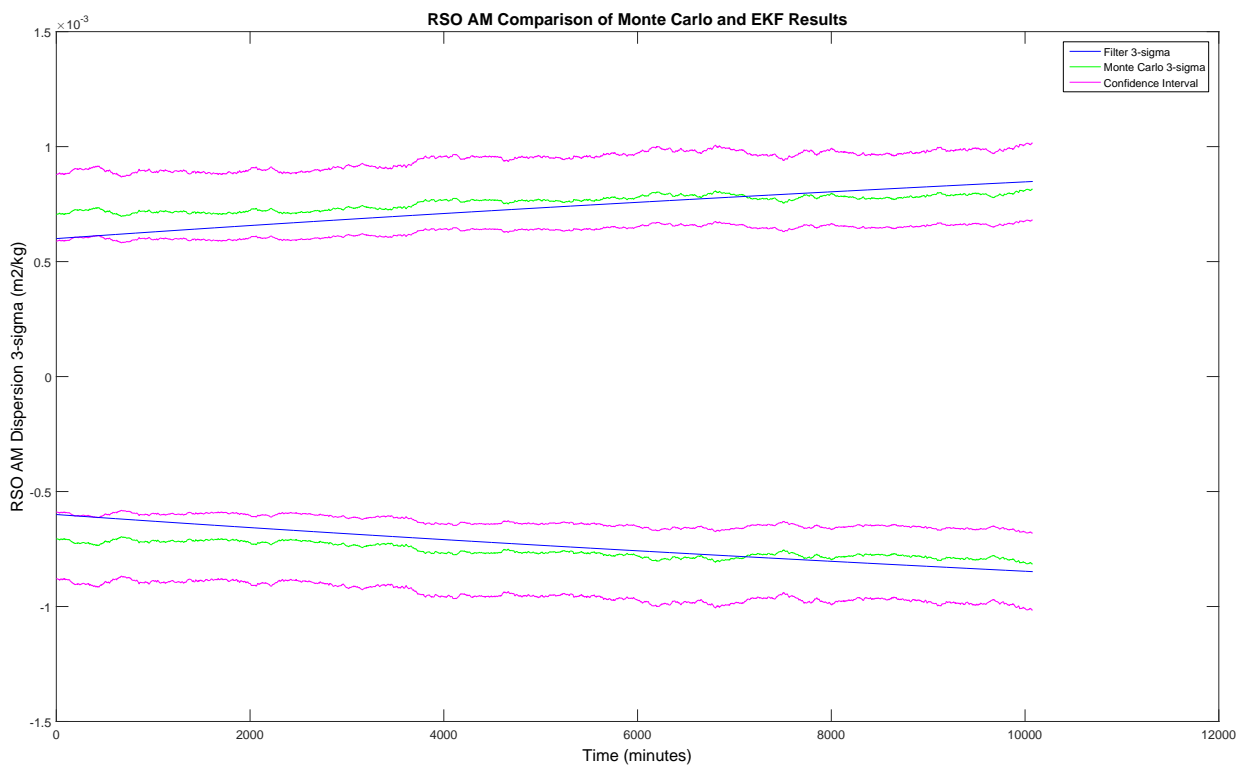

Fig. 7.32: Low Initial Errors - Ground Station Only Measurements, EKF Validation, RSO Area-to-Mass Ratio Estimation Error 

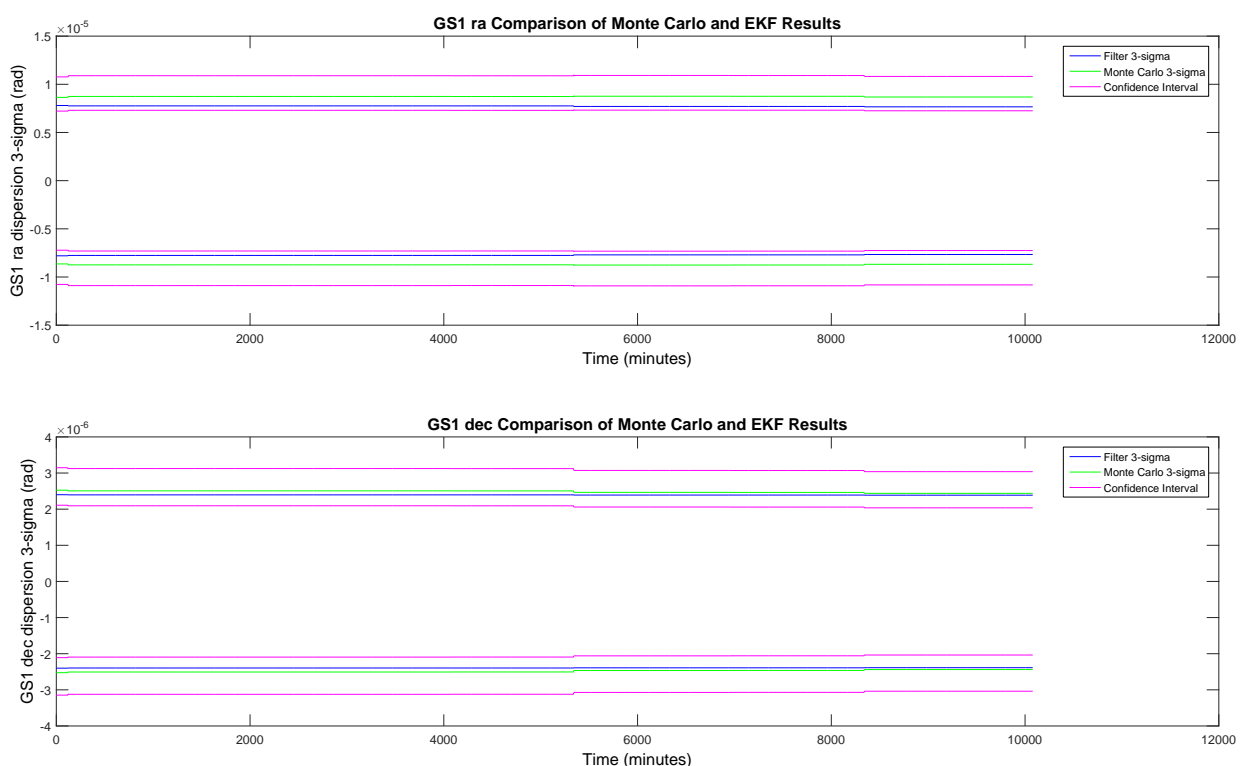

Fig. 7.33: Low Initial Errors - Ground Station Only Measurements, EKF Validation, Ground Station 1 Biases Estimation Errors
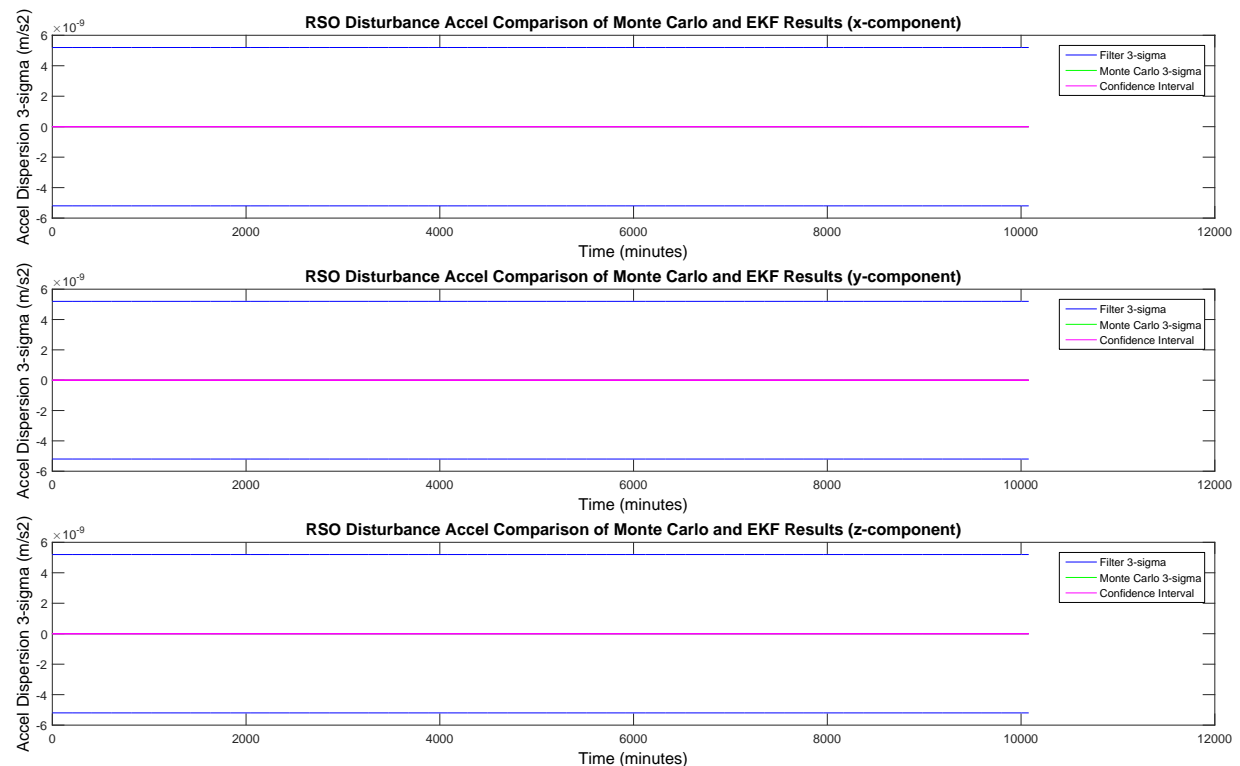

Fig. 7.34: Low Initial Errors - Ground Station Only Measurements, EKF Validation, RSO Disturbance Accels Estimation Errors 


\subsubsection{Low Circular Space Sensor Orbit}

Figures 7.35 through 7.45 are results from a 50 run analysis of the RSO with low circular orbit space sensor and ground station measurements. All error sources are set to the low level. The main differences between these results and the results from the truth model $2 \times 2$ gravity analysis are seen in the disturbance acceleration plots of the RSO and space sensor. It can be seen that the EKF disturbance acceleration lies outside of the Monte Carlo confidence intervals. The EKF disturbance acceleration model was built to be conservative. The EKF slightly overestimates the effect of the disturbance accelerations in all scenarios.
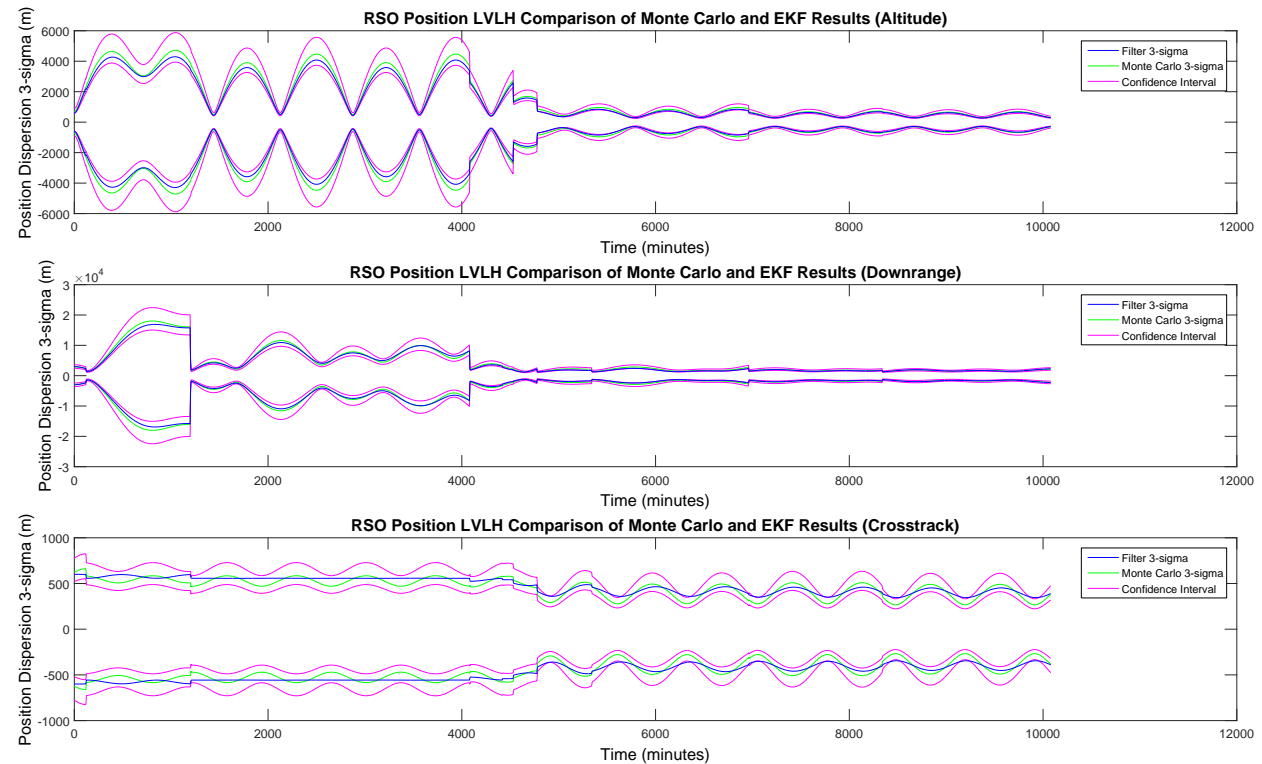

Fig. 7.35: Low Initial Errors - Ground Station and Low Circular SS Measurements, EKF Validation, RSO LVLH Position Estimation Errors 

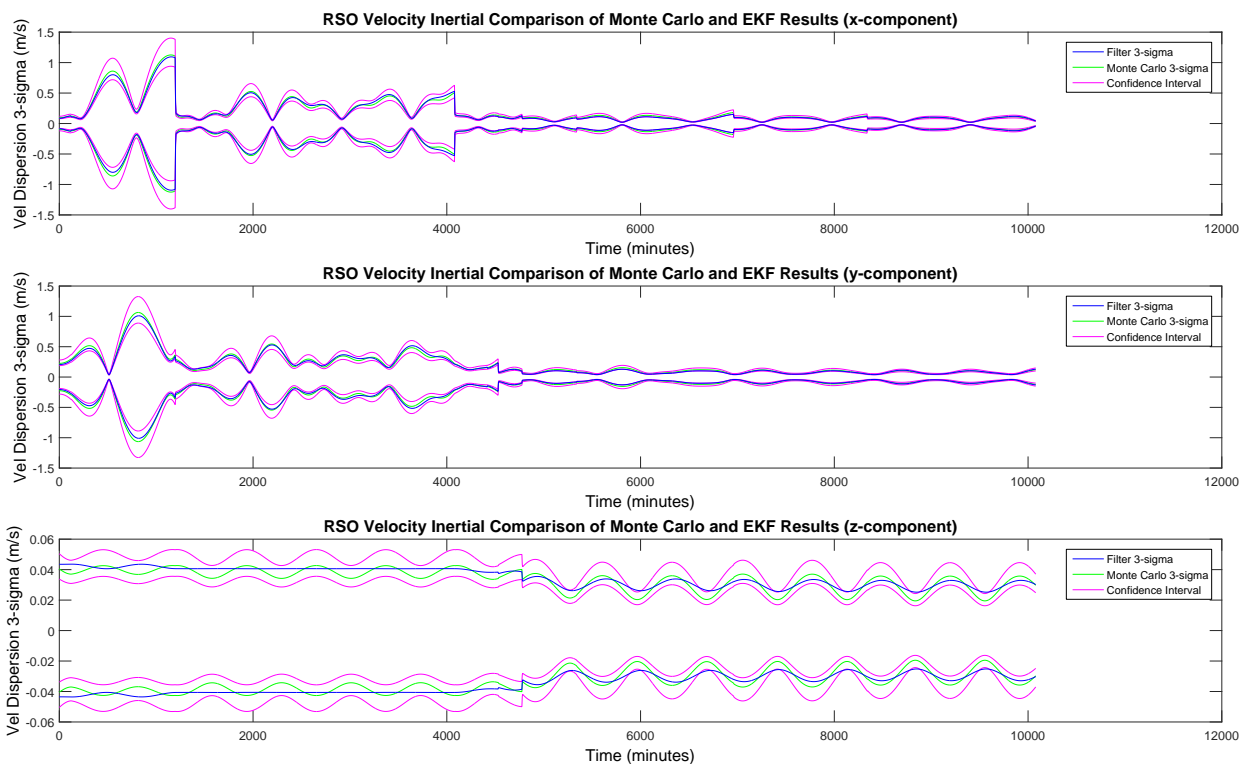

Fig. 7.36: Low Initial Errors - Ground Station and Low Circular SS Measurements, EKF Validation, RSO Inertial Velocity Estimation Errors

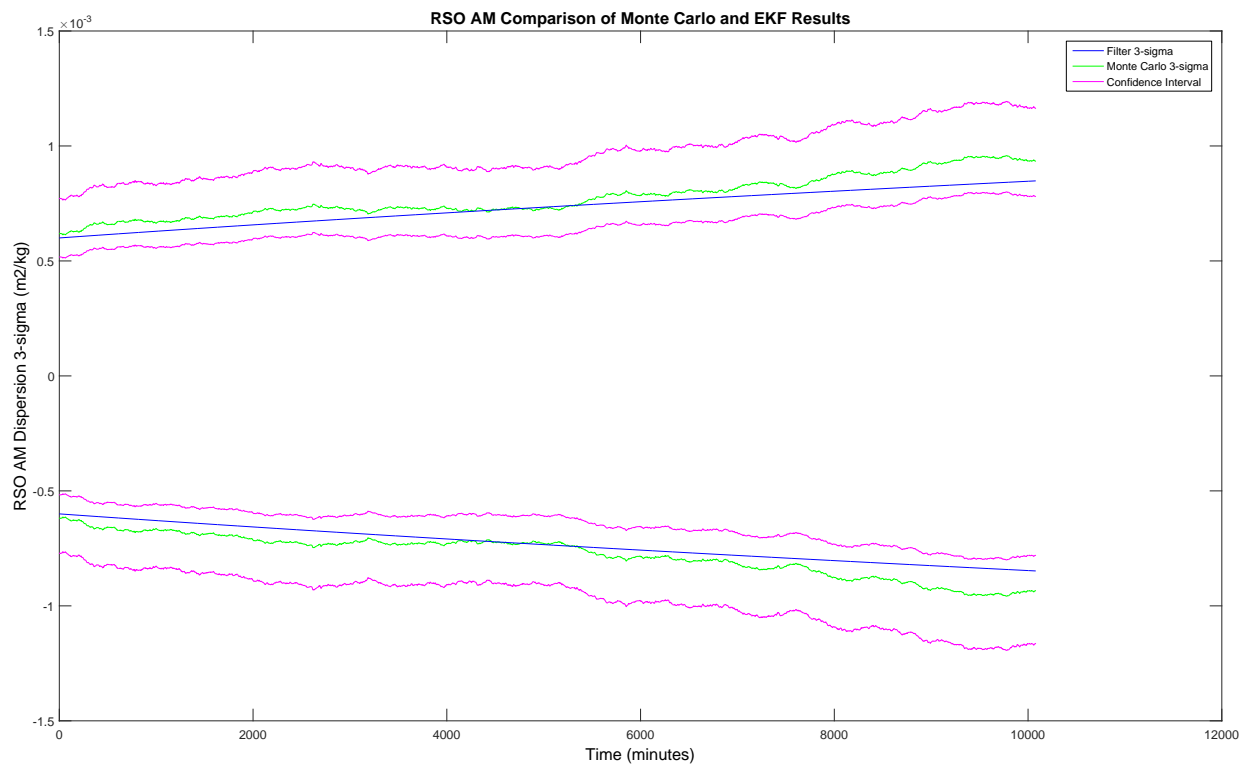

Fig. 7.37: Low Initial Errors - Ground Station and Low Circular SS Measurements, EKF Validation, RSO Area-to-Mass Ratio Estimation Error 

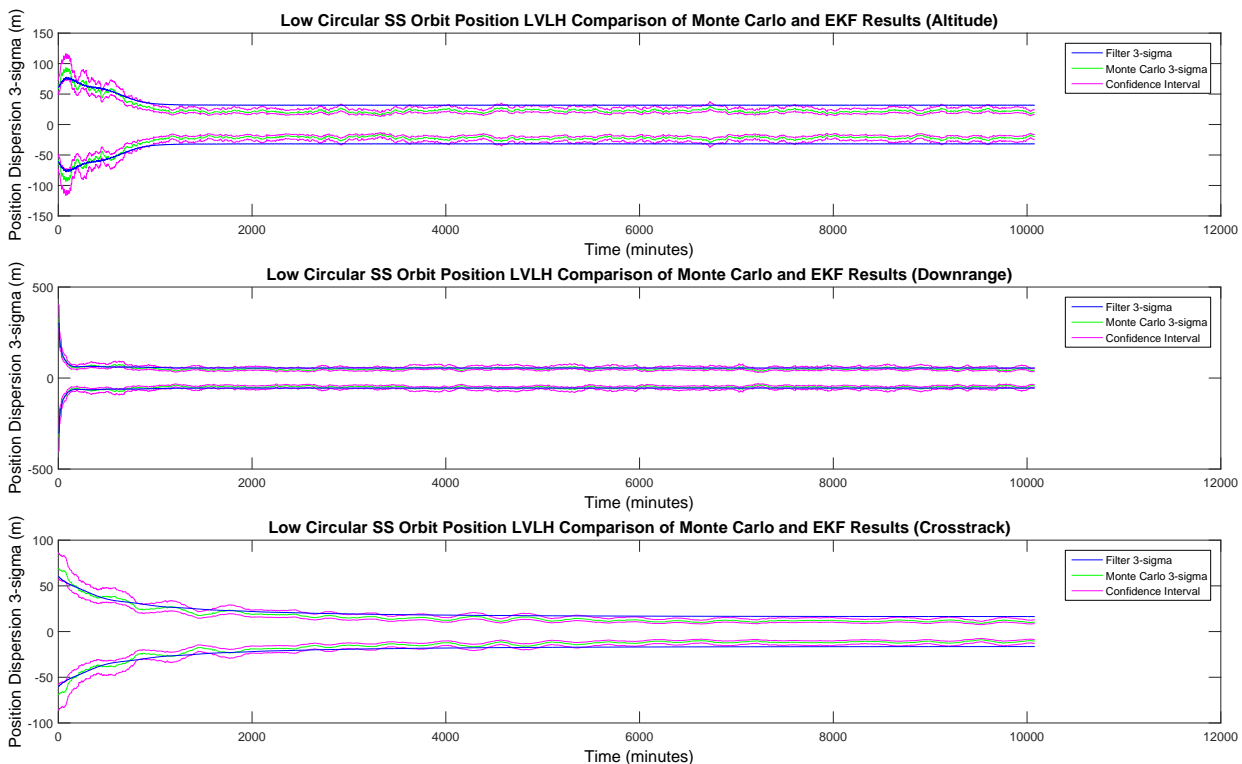

Fig. 7.38: Low Initial Errors - Ground Station and Low Circular SS Measurements, EKF Validation, SS LVLH Position Estimation Errors
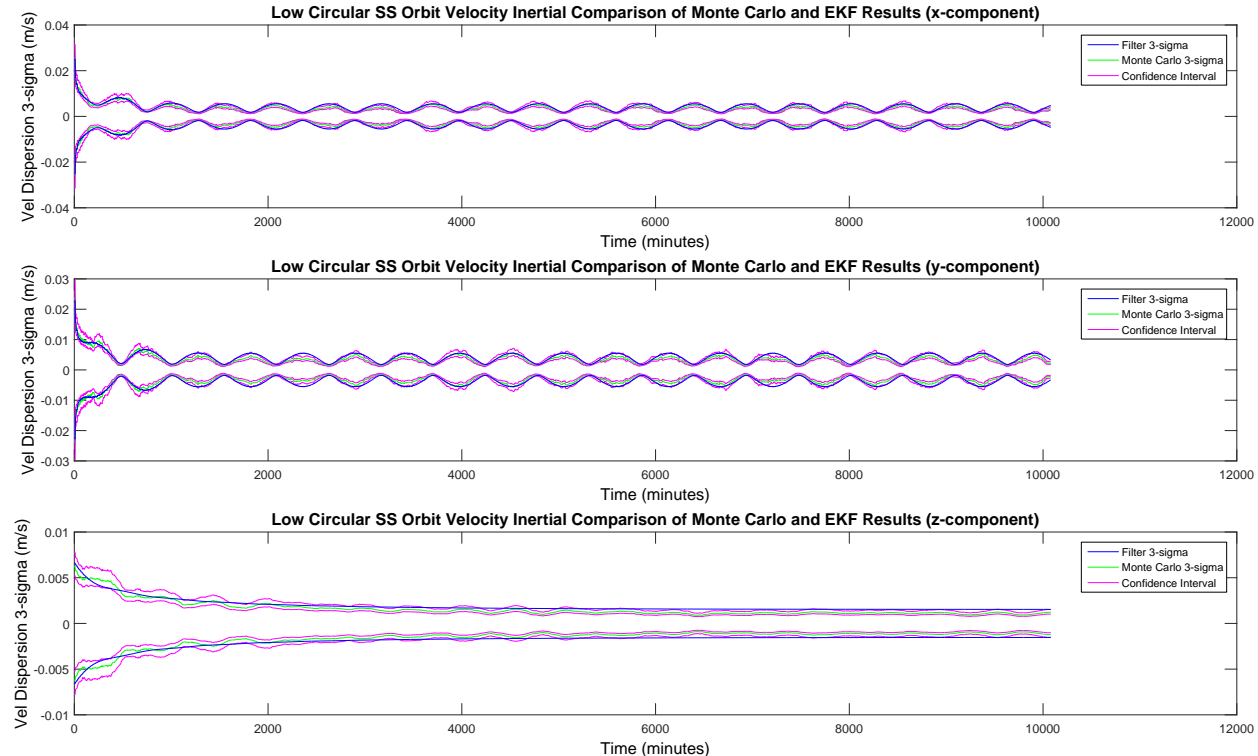

Fig. 7.39: Low Initial Errors - Ground Station and Low Circular SS Measurements, EKF Validation, SS Inertial Velocity Estimation Errors 


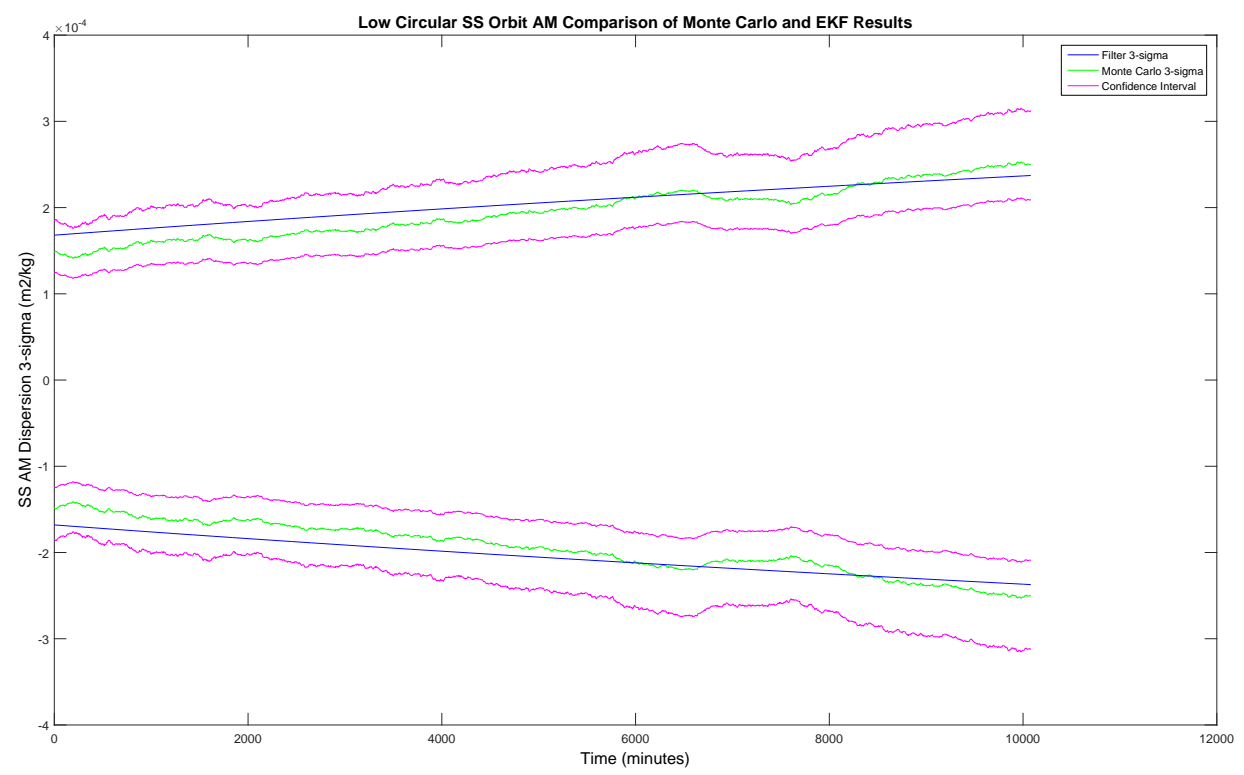

Fig. 7.40: Low Initial Errors - Ground Station and Low Circular SS Measurements, EKF Validation, SS Area-to-Mass Ratio Estimation Error
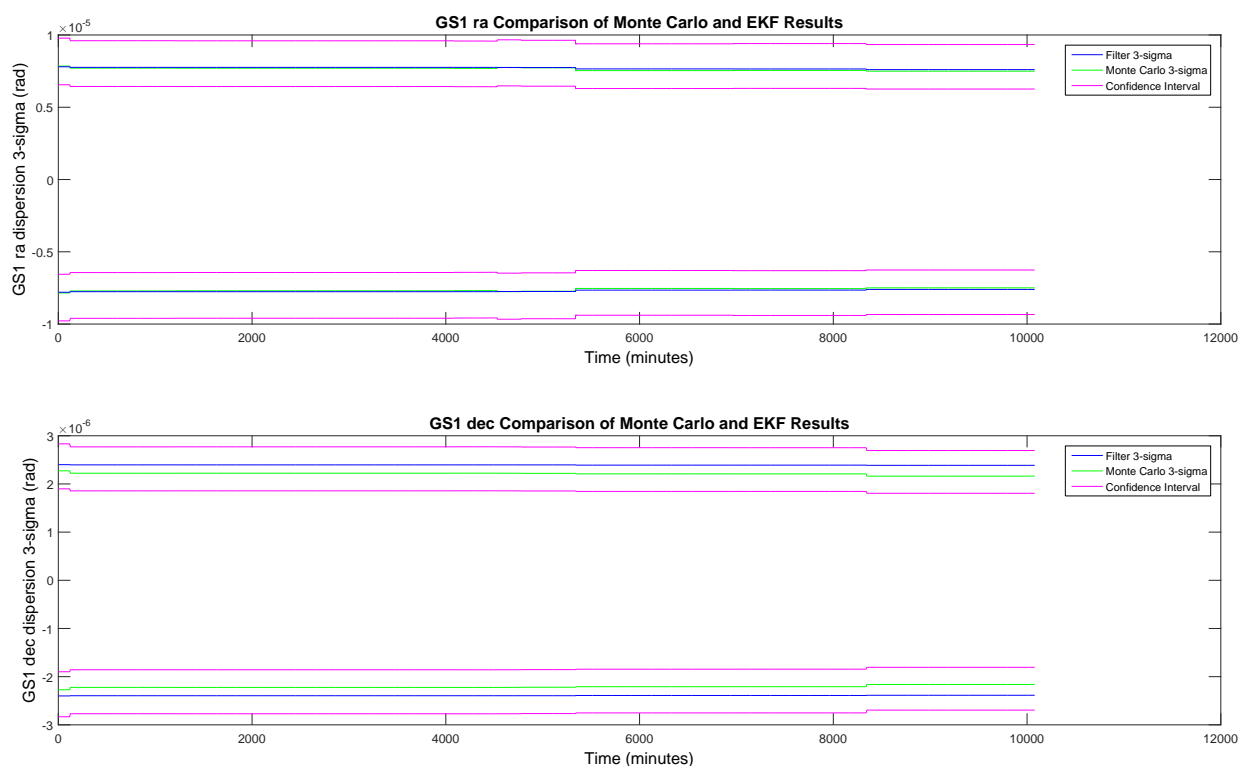

Fig. 7.41: Low Initial Errors - Ground Station and Low Circular SS Measurements, EKF Validation, Ground Station 1 Biases Estimation Errors 

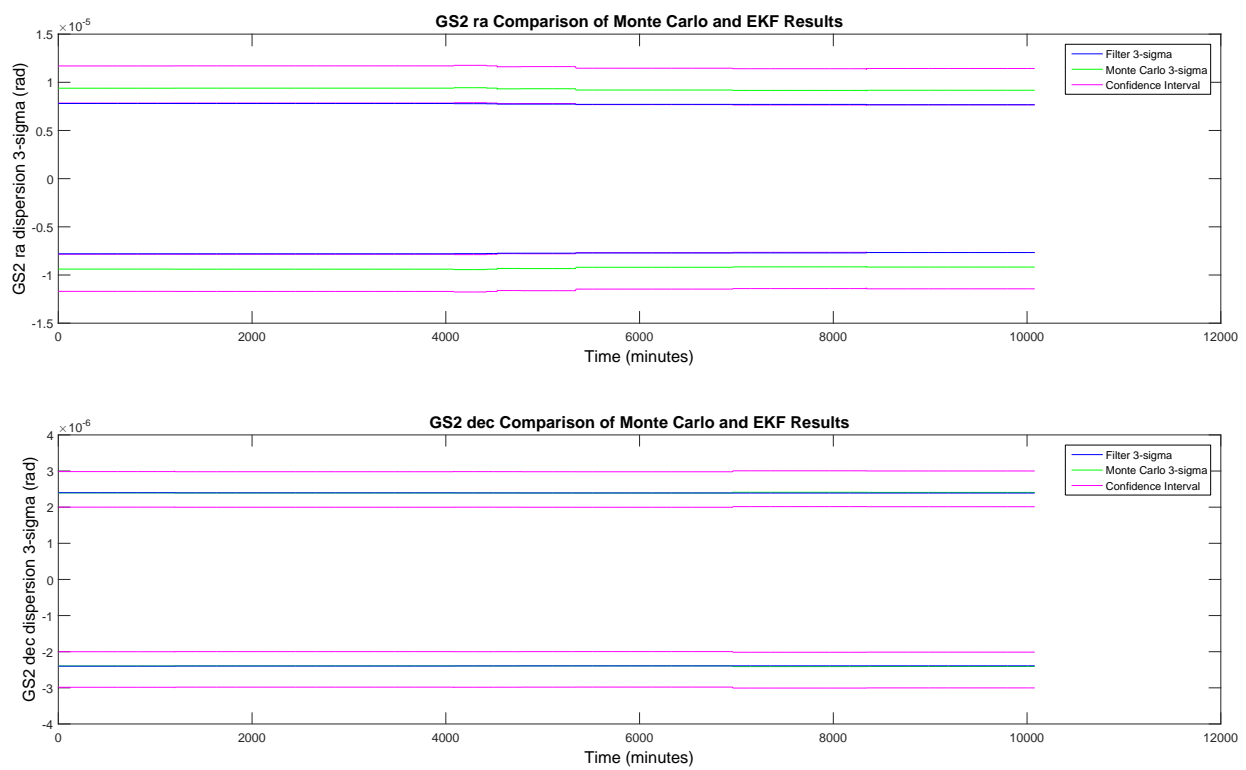

Fig. 7.42: Low Initial Errors - Ground Station and Low Circular SS Measurements, EKF Validation, Ground Station 2 Biases Estimation Errors
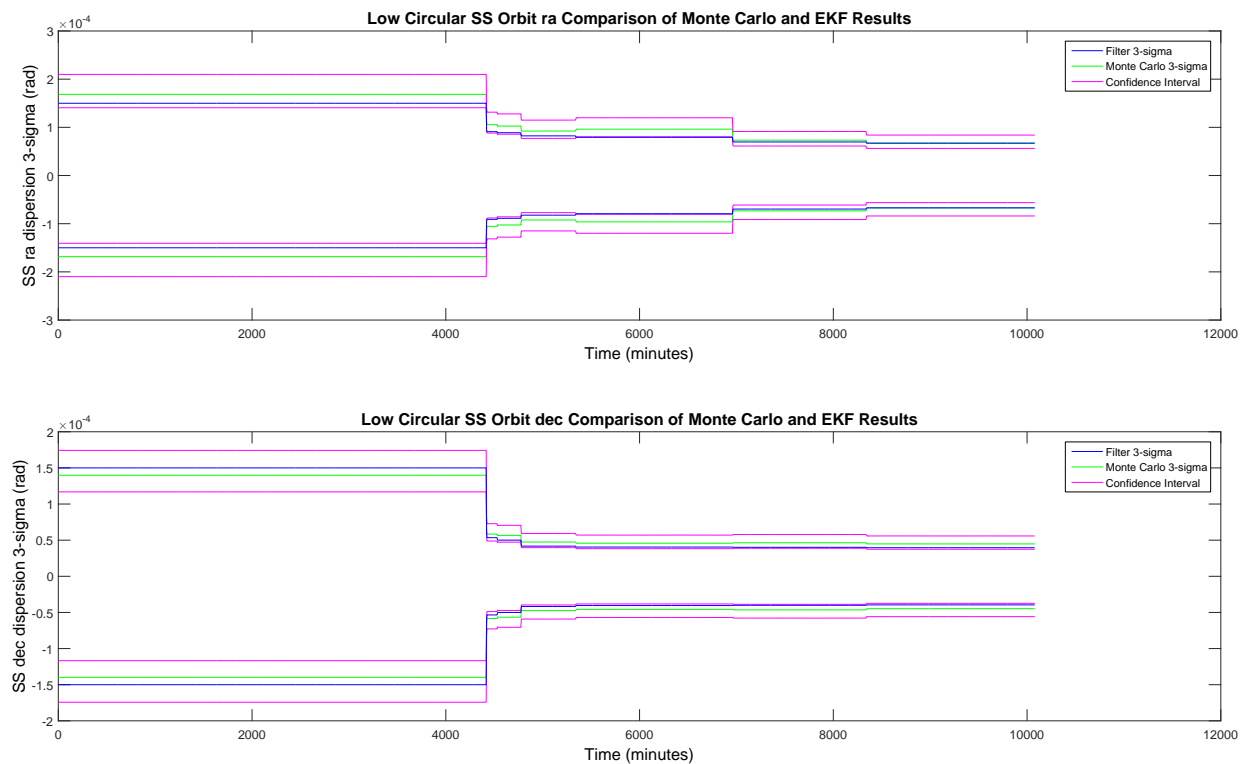

Fig. 7.43: Low Initial Errors - Ground Station and Low Circular SS Measurements, EKF Validation, SS Biases Estimation Errors 

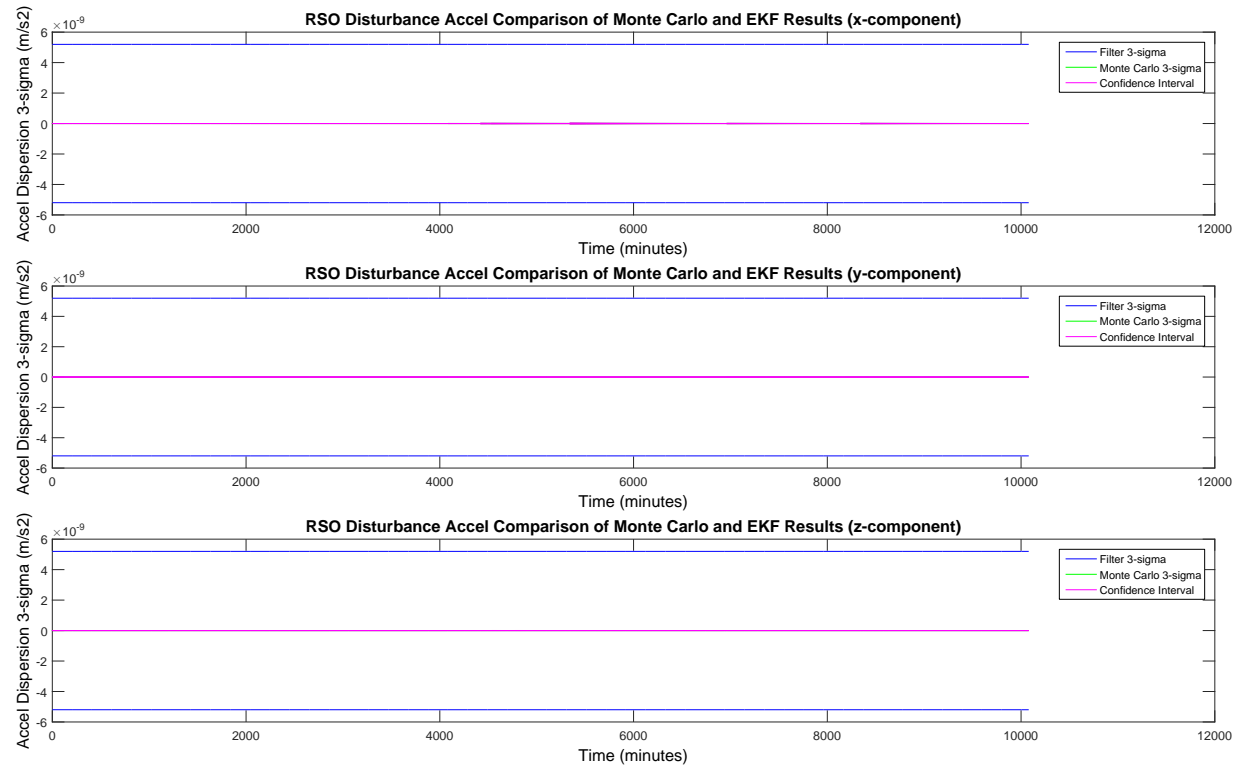

Fig. 7.44: Low Initial Errors - Ground Station and Low Circular SS Measurements, EKF Validation, RSO Disturbance Accels Estimation Errors
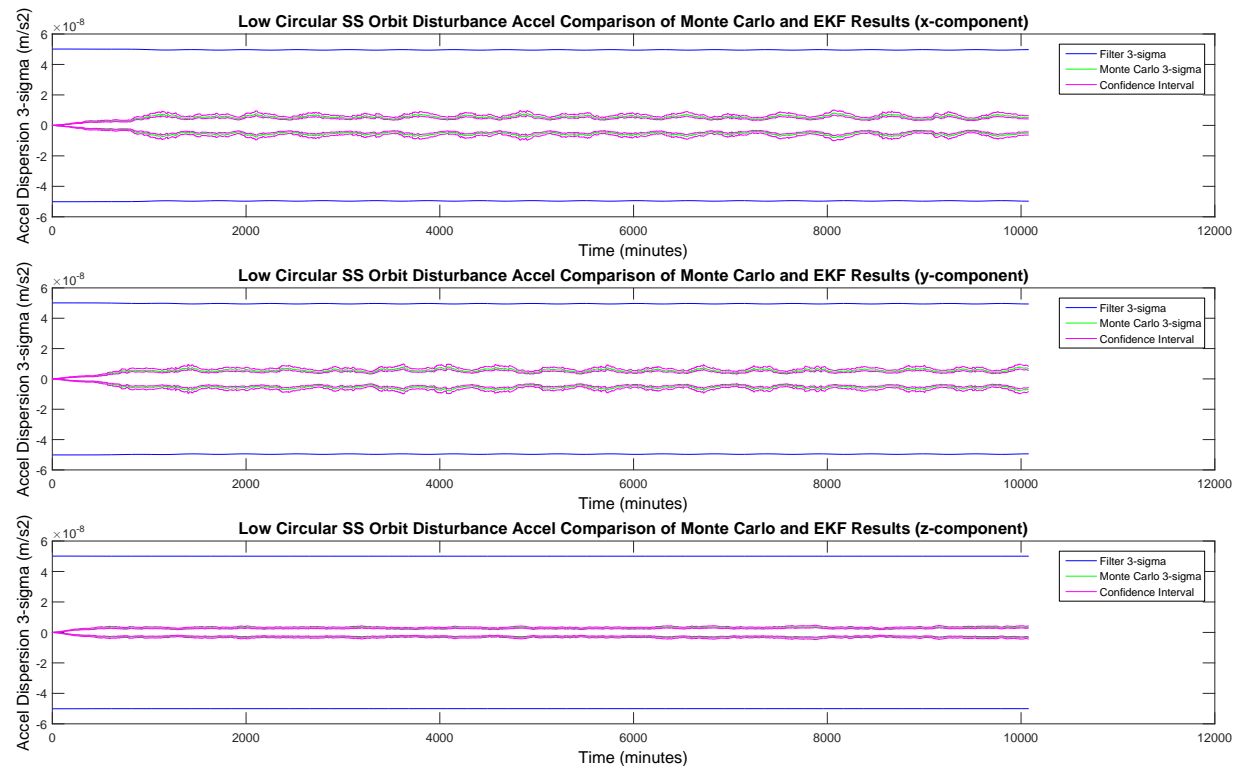

Fig. 7.45: Low Initial Errors - Ground Station and Low Circular SS Measurements, EKF Validation, SS Disturbance Accels Estimation Errors 


\subsubsection{High Circular Space Sensor Orbit}

Figures 7.46 and 7.47 are results from a 50 run analysis of the RSO with high circular orbit space sensor and ground station measurements. All error sources are set to the low level. The results were comparable to the low circular scenario. The EKF slightly overestimates the effect of the disturbance accelerations.
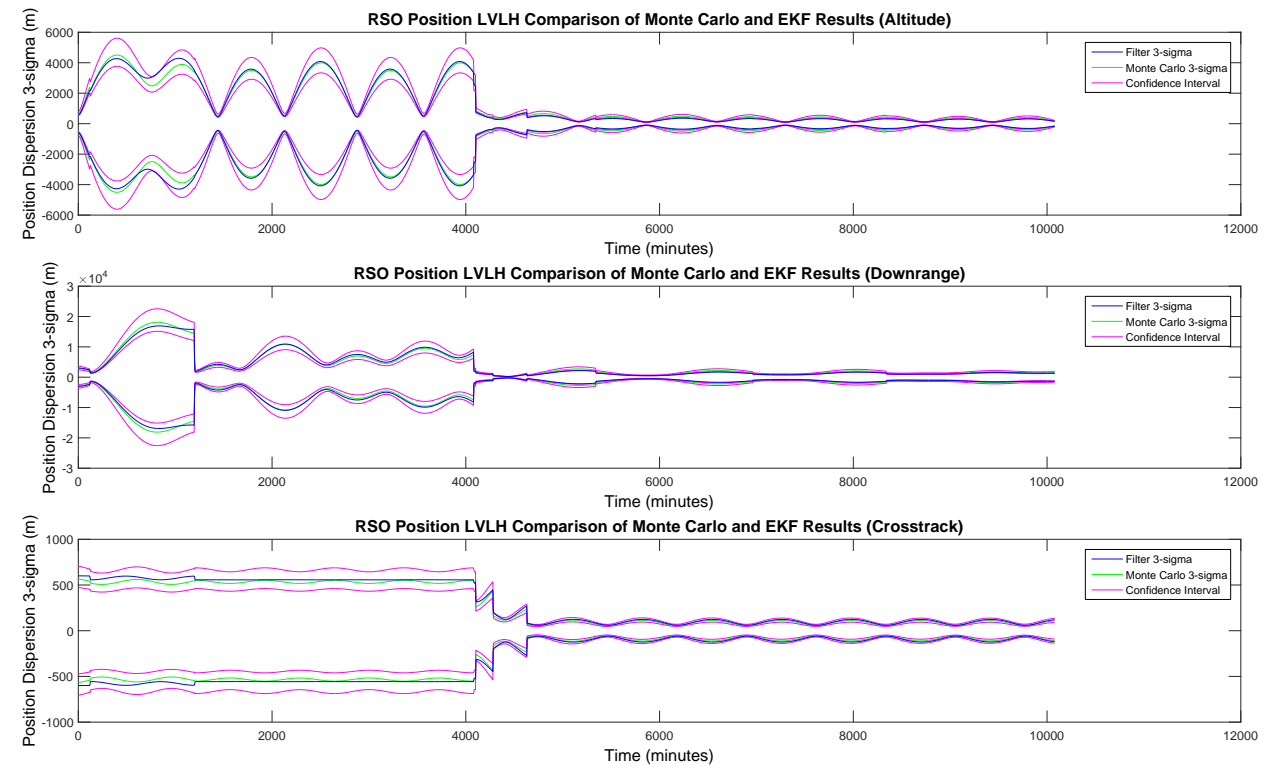

Fig. 7.46: Low Initial Errors - Ground Station and High Circular SS Measurements, EKF Validation, RSO LVLH Position Estimation Errors 

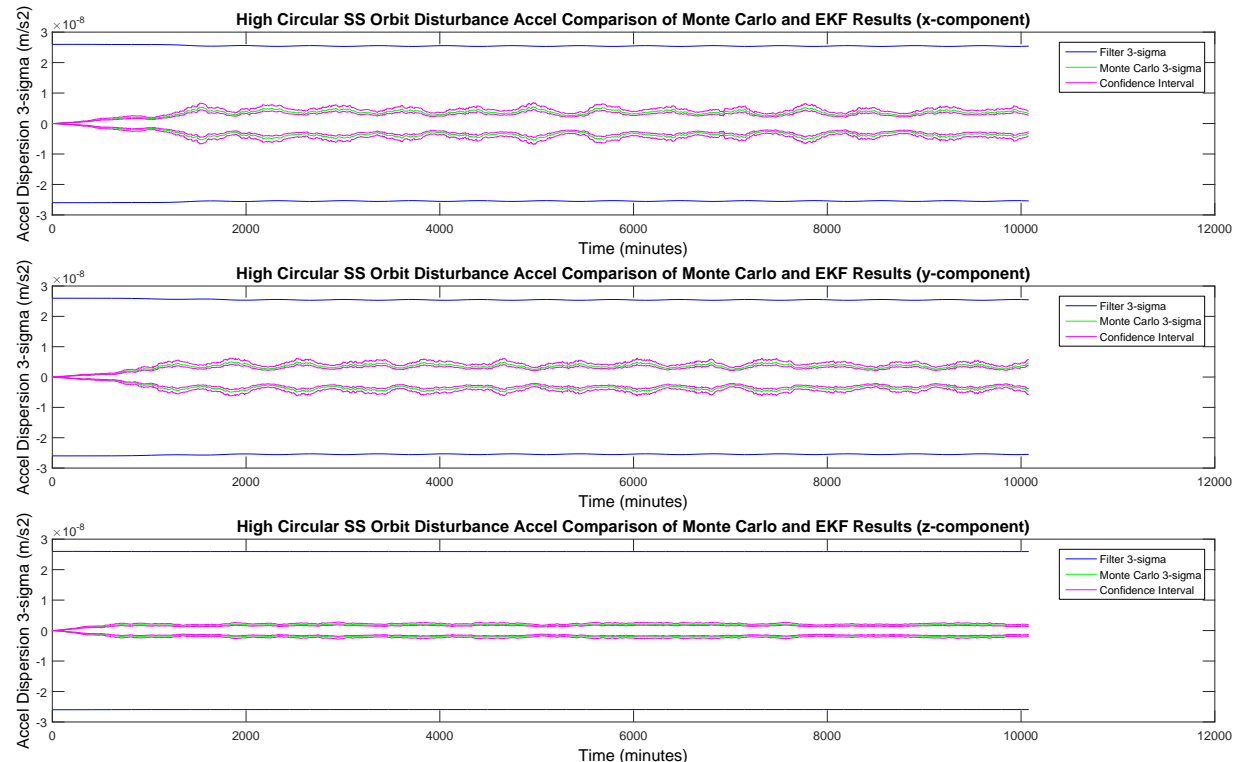

Fig. 7.47: Low Initial Errors - Ground Station and High Circular SS Measurements, EKF Validation, SS Disturbance Accels Estimation Errors

\subsubsection{Elliptical Space Sensor Orbit}

Figures 7.48 and 7.49 are results from a 50 run analysis of the RSO with elliptical orbit space sensor and ground station measurements. All error sources are set to the low level. The results were comparable to the low circular scenario. The EKF slightly overestimates the effect of the disturbance accelerations. 

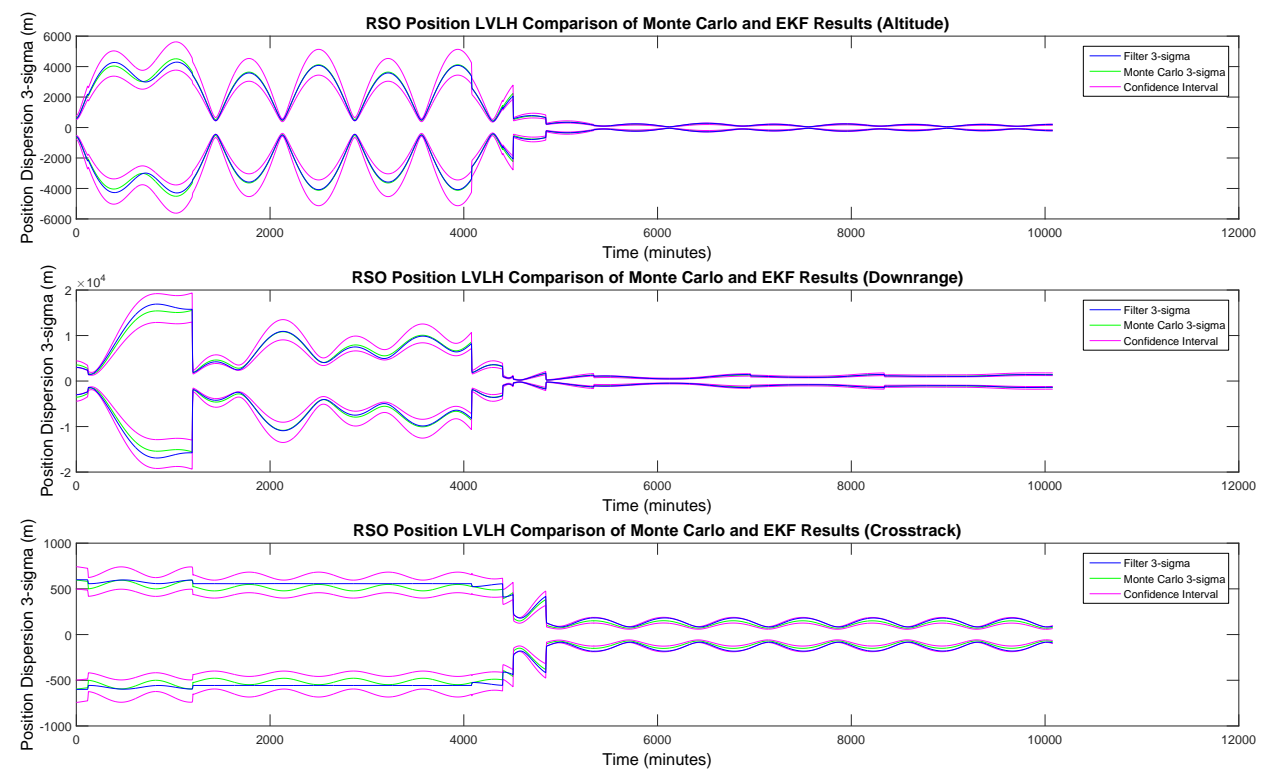

Fig. 7.48: Low Initial Errors - Ground Station and Elliptical SS Measurements, EKF Validation, RSO LVLH Position Estimation Errors
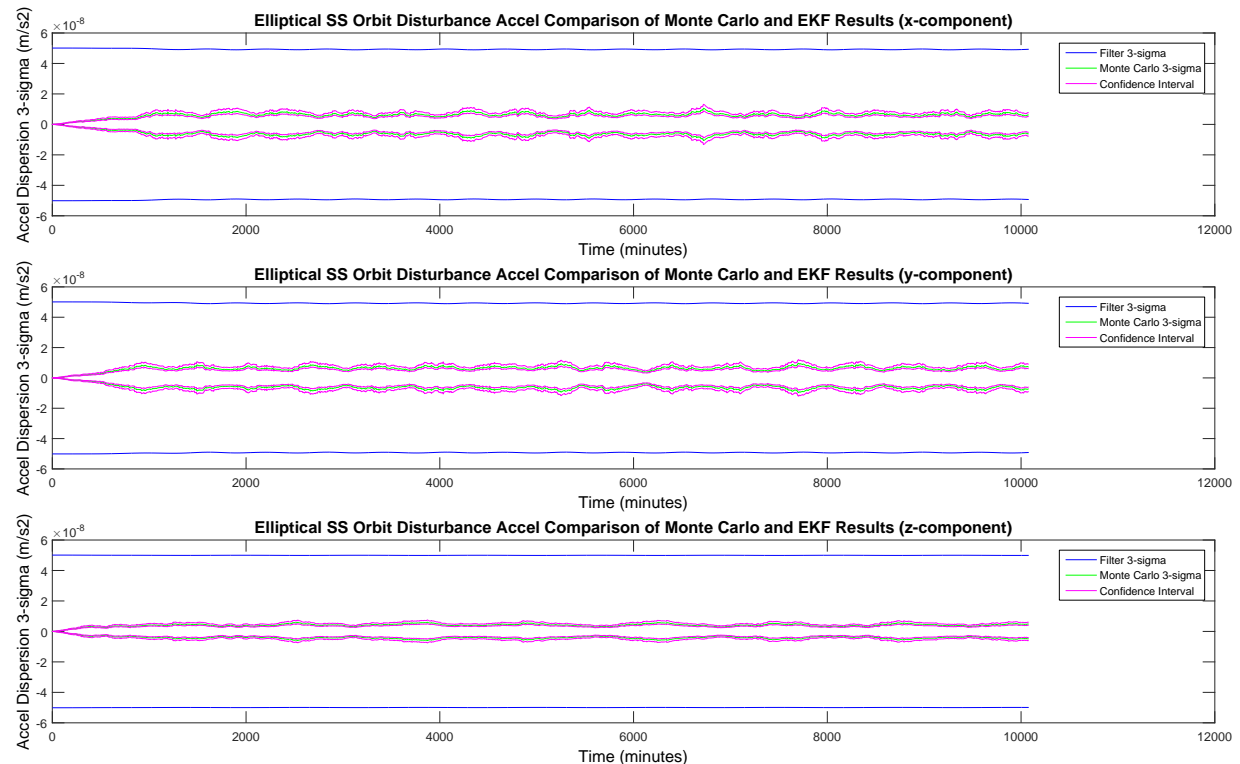

Fig. 7.49: Low Initial Errors - Ground Station and Elliptical SS Measurements, EKF Validation, SS Disturbance Accels Estimation Errors 


\subsection{Summary}

This chapter validated the developed EKF for the RSO estimation problem through Monte Carlo analysis. This EKF can now be used in the development of the LinCov analysis tool. 
CHAPTER 8

\section{LINEAR COVARIANCE ANALYSIS TOOL DEVELOPMENT}

\subsection{LinCov Overview}

This chapter covers the equations and development of the LinCov analysis tool that is used for the analysis and results portion of this research. First, the fundamental equations are provided, followed by the specific variables and matrices for the RSO estimation problem, and finally a discussion on how this tool can be used for error budget analysis.

The equations presented in Chapters 4 and 5 for the truth model and extended Kalman filter are linearized around a mean reference trajectory, $\overline{\boldsymbol{x}}$, to obtain the set of equations for LinCov. These equations describe the true and filter state dispersions, $\delta \boldsymbol{x}$ and $\delta \hat{\boldsymbol{x}}$.

The true state propagation equation, Equation 4.1, and the EKF state propagation equation, Equation 5.1, are linearized to produce the LinCov true and filter state dispersion propagation Equations, 8.1 and 8.2.

$$
\begin{gathered}
\delta \dot{\boldsymbol{x}}=F_{\boldsymbol{x}} \delta \boldsymbol{x}+\boldsymbol{w} \\
\delta \dot{\hat{\boldsymbol{x}}}=\hat{F}_{\hat{\boldsymbol{x}}} \delta \hat{\boldsymbol{x}}
\end{gathered}
$$

where:

$$
\begin{gathered}
F_{\boldsymbol{x}}=\left.\frac{\partial f}{\partial \boldsymbol{x}}\right|_{\overline{\boldsymbol{x}}} \\
\hat{F}_{\hat{\boldsymbol{x}}}=\left.\frac{\partial \hat{f}}{\partial \hat{\boldsymbol{x}}}\right|_{\overline{\boldsymbol{x}}}
\end{gathered}
$$

these partial derivatives are evaluated along the reference trajectory rather than the current state estimate as in the EKF.

The true state update is simply 


$$
\delta \boldsymbol{x}^{+}=\delta \boldsymbol{x}^{-}
$$

The EKF state update equation is linearized to produce

$$
\delta \hat{\boldsymbol{x}}_{k}^{+}=\hat{K}_{k} H_{k} \delta \mathbf{x}_{k}^{-}+\hat{K}_{k} \nu_{k}+\left[I-\hat{K}_{k} \hat{H}_{k}\right] \delta \hat{\mathbf{x}}_{k}^{-}
$$

The Kalman gain is evaluated in the same manner as the Monte Carlo EKF:

$$
\hat{K}_{k}=\hat{P}_{k}^{-} \hat{H}_{k}^{T}\left(\hat{H}_{k} \hat{P}_{k}^{-} \hat{H}_{k}^{T}+\hat{R}_{v}\right)^{-1}
$$

where:

$$
\begin{aligned}
& H_{k}=\left.\frac{\partial \boldsymbol{h}(\boldsymbol{x}, t)}{\partial \boldsymbol{x}}\right|_{\overline{\boldsymbol{x}}} \\
& \hat{H}_{k}=\left.\frac{\partial \hat{\boldsymbol{h}}(\hat{\boldsymbol{x}}, t)}{\partial \hat{\boldsymbol{x}}}\right|_{\overline{\boldsymbol{x}}}
\end{aligned}
$$

Again, these partial derivatives are evaluated along the reference trajectory rather than the current state estimate as in the EKF.

The filter state covariance is determined using the equations from the Monte Carlo EKF:

$$
\begin{gathered}
\hat{P}_{i+1}^{+}=\Phi_{i} \hat{P}_{i} \Phi_{i}^{T}+\hat{Q}_{d, i} \\
\hat{P}_{k}^{+}=\left(I-\hat{K}_{k} \hat{H}_{k}\right) \hat{P}_{k}^{-}\left(I-\hat{K}_{k} \hat{H}_{k}\right)^{T}+\hat{K}_{k} \hat{R}_{v} \hat{K}_{k}^{T}
\end{gathered}
$$

The augmented LinCov state vector is created from the state dispersions.

$$
\boldsymbol{X}=\left[\begin{array}{l}
\delta \boldsymbol{x} \\
\delta \hat{\boldsymbol{x}}
\end{array}\right]
$$


Now, the LinCov state propagation and update equations can be formed from Equations $8.1,8.2$, and 8.6 .

$$
\dot{\boldsymbol{X}}=\mathcal{F} \boldsymbol{X}+\mathcal{W} \boldsymbol{w}
$$

$$
\boldsymbol{X}_{k}^{+}=\mathcal{A}_{k} \boldsymbol{X}_{k}^{-}+\mathcal{B}_{k} \nu_{k}
$$

where

$$
\begin{gathered}
\mathcal{F}=\left[\begin{array}{cc}
F_{\boldsymbol{x}} & 0_{n \times n} \\
0_{n \times n} & \hat{F}_{\hat{\boldsymbol{x}}}
\end{array}\right] \quad \mathcal{W}=\left[\begin{array}{c}
I_{n \times n} \\
0_{\hat{n} \times n}
\end{array}\right] \quad \mathcal{B}=\left[\begin{array}{c}
0_{n \times n_{z}} \\
\hat{K}_{k} \\
\mathcal{A}_{k}
\end{array}\right]
\end{gathered}
$$

The nominal trajectory of this linear system is the mean trajectory from the Monte Carlo simulation. Therefore the expected value of the state dispersions is zero, as shown in Eq. 8.17 and Eq. 8.18 .

$$
\begin{aligned}
& E[\delta \boldsymbol{x}]=E[\boldsymbol{x}-\overline{\boldsymbol{x}}]=E[\boldsymbol{x}]-\overline{\boldsymbol{x}}=0 \\
& E[\delta \hat{\boldsymbol{x}}]=E[\hat{\boldsymbol{x}}-\overline{\boldsymbol{x}}]=E[\hat{\boldsymbol{x}}]-\overline{\boldsymbol{x}}=0
\end{aligned}
$$

This allows the covariance of the LinCov augmented state vector to be found directly from the state vector

$$
C_{A}=E\left[\boldsymbol{X}(t) \boldsymbol{X}^{T}(t)\right]
$$

The covariance is propagated using 


$$
C_{A, i+1}=\Phi_{i} C_{A, i} \Phi_{i}^{T}+S \triangle t
$$

where the state transition matrix, $\Phi$, to the second order, is defined as

$$
\Phi=e^{\mathcal{F} \Delta t}=I_{n x n}+\mathcal{F} \Delta t+\mathcal{F} \mathcal{F} \Delta t^{2} / 2 !
$$

and

$$
S=\mathcal{W} S_{w} \mathcal{W}^{T}=\left[\begin{array}{cc}
S_{w} & 0_{n \times \hat{n}} \\
0_{\hat{n} \times n} & 0_{\hat{n} \times \hat{n}}
\end{array}\right]
$$

The covariance is updated using

$$
C_{A}\left(t_{k}^{+}\right)=\mathcal{A}_{k} C_{A}\left(t_{k}^{-}\right) \mathcal{A}_{k}^{T}+\mathcal{B}_{k} R_{v} \mathcal{B}_{k}^{T}
$$

The covariance for LinCov analysis is initialized as:

$$
C_{A}\left(t_{0}\right)=\left[\begin{array}{cc}
E\left[\delta \boldsymbol{x}_{0} \delta \boldsymbol{x}_{0}^{T}\right] & 0_{n \times \hat{n}} \\
0_{\hat{n} \times n} & E\left[\delta \hat{\boldsymbol{x}}_{0} \delta \hat{\boldsymbol{x}}_{0}^{T}\right]
\end{array}\right]
$$

where

$$
\begin{gathered}
E\left[\delta \boldsymbol{x}_{0} \delta \boldsymbol{x}_{0}^{T}\right]=P_{\boldsymbol{x} \boldsymbol{x}}\left(t_{0}\right) \\
E\left[\delta \hat{\boldsymbol{x}}_{0} \delta \hat{\boldsymbol{x}}_{0}^{T}\right]=0_{\hat{n} \times \hat{n}}
\end{gathered}
$$

\subsection{LinCov Models}

The truth dynamics for the LinCov model are taken from the truth model as presented in Chapter 4, using a $2 \times 2$ spherical gravity model and the disturbance accelerations to 
incorporate higher-order gravity terms. The filter algorithm used in the LinCov model is taken from the EKF developed in Chapter 5. The partial derivative matrices required to propagate and update the LinCov covariance are presented in the sections below.

\subsubsection{LinCov Covariance Propagation}

To propagate the LinCov covariance through use of the state transition matrix, given in Eq. 8.20, the matrix, $\mathcal{F}$, which consists of the Jacobians, $F_{\boldsymbol{x}}$ and $\hat{F}_{\hat{\boldsymbol{x}}}$, must be defined.

For this analysis, the Jacobian $F_{\boldsymbol{x}}$ is:

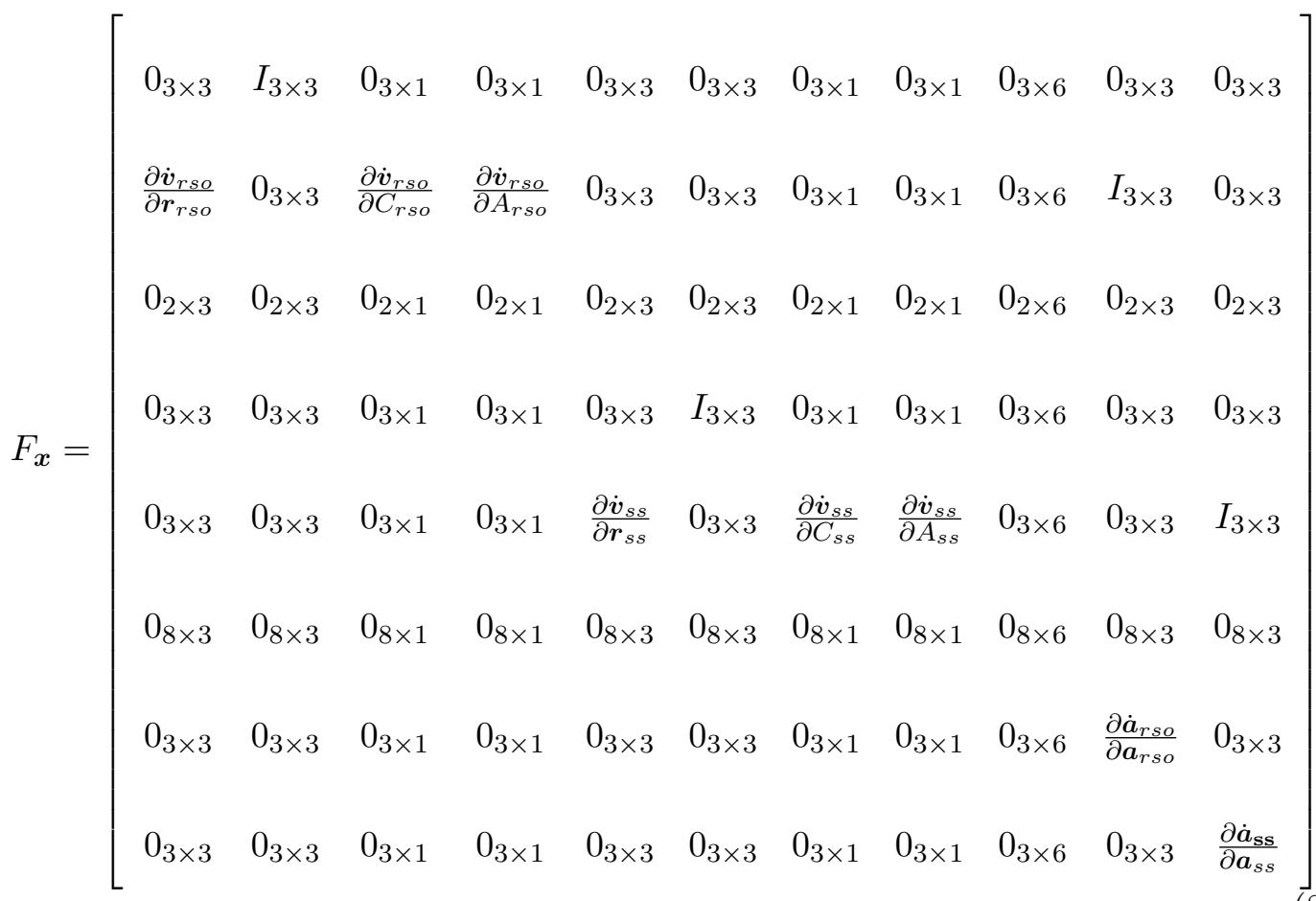

where:

$$
\begin{gathered}
\frac{\partial \dot{\boldsymbol{v}}}{\partial C}=-P_{S R P}(A)\left(\frac{\rho_{\text {Sun }}-\boldsymbol{r}}{\left\|\rho_{\text {Sun }}-\boldsymbol{r}\right\|}\right) \\
\frac{\partial \dot{\boldsymbol{v}}}{\partial A}=-P_{S R P}(C)\left(\frac{\rho_{\text {Sun }}-\boldsymbol{r}}{\left\|\rho_{\text {Sun }}-\boldsymbol{r}\right\|}\right) \\
\frac{\partial \dot{\boldsymbol{a}}}{\partial \boldsymbol{a}}=\frac{-1}{\tau} I_{3 x 3}
\end{gathered}
$$


and

$$
\frac{\partial \dot{\boldsymbol{v}}}{\partial \boldsymbol{r}}=\frac{\partial \boldsymbol{a}_{2,2}}{\partial \boldsymbol{r}}+\frac{\partial \boldsymbol{a}_{\text {moon }}}{\partial \boldsymbol{r}}+\frac{\partial \boldsymbol{a}_{\text {sun }}}{\partial \boldsymbol{r}}+\frac{\partial \boldsymbol{a}_{S R P}}{\partial \boldsymbol{r}} \hat{H}_{\hat{\boldsymbol{x}}}
$$

where

$$
\begin{gathered}
\frac{\partial \boldsymbol{a}_{2,2}}{\partial \boldsymbol{r}}=-\frac{\mu}{r^{3}}\left(-3 \hat{i}^{T}+I_{3 x 3}\right)-\frac{\mu(J 2)\left(R_{\oplus}^{2}\right)}{2} \\
\left\{\left(\frac{-30\left(\boldsymbol{n}^{T} \boldsymbol{r}\right) \boldsymbol{n}\left(\hat{i}^{T}\right)}{r^{6}}\right)+\left(\frac{6\left(\boldsymbol{n} \boldsymbol{n}^{T}\right)}{r^{5}}\right)-\left(\frac{15 \boldsymbol{r}\left(\hat{i}^{T}\right)}{r^{6}}\right)+\left(\frac{3\left(I_{3 x 3}\right)}{r^{5}}\right)+\right. \\
\left.\left(\frac{75\left(\hat{i}^{T} \boldsymbol{n}\right)^{2} \boldsymbol{r}\left(\hat{i}^{T}\right)}{r^{6}}\right)-\left(\frac{15\left(\hat{i}^{T} \boldsymbol{n}\right) I_{3 x 3}}{r^{5}}\right)-\left(\frac{30 \boldsymbol{r}\left(\boldsymbol{n}^{T} \hat{i}\right) \boldsymbol{n}^{T}}{r^{6}}\right)\left(I_{3 x 3}-\hat{i}^{T}\right)\right\} \\
\boldsymbol{n}=\left[\begin{array}{lll}
0 & 0 & 1
\end{array}\right]^{T}
\end{gathered}
$$

Note: This partial derivative includes only $J_{2,0}$ and does not include the partial derivative for $J_{2,2}$.

$$
\begin{gathered}
\frac{\partial \boldsymbol{a}_{\text {moon }}}{\partial \boldsymbol{r}}=\left(3 \mu_{\text {Moon }} \frac{\boldsymbol{r}-\rho_{\text {Moon }}}{\left\|\boldsymbol{r}-\rho_{\text {Moon }}\right\|^{4}} \frac{\left(\boldsymbol{r}-\rho_{\text {Moon }}\right)^{T}}{\left\|\boldsymbol{r}-\rho_{\text {Moon }}\right\|}\right)-\frac{\mu_{\text {Moon }}}{\| \boldsymbol{r}-\rho_{\text {Moon } \|^{3}} I_{3 x 3}} \\
\frac{\partial \boldsymbol{a}_{\text {sun }}}{\partial \boldsymbol{r}}=\left(3 \mu_{\text {Sun }} \frac{\boldsymbol{r}-\rho_{\text {Sun }}}{\left\|\boldsymbol{r}-\rho_{\text {Sun }}\right\|^{4}} \frac{\left(\boldsymbol{r}-\rho_{\text {Sun }}\right)^{T}}{\left\|\boldsymbol{r}-\rho_{\text {Sun }}\right\|}\right)-\frac{\mu_{\text {Sun }}}{\left\|\boldsymbol{r}-\rho_{\text {Sun }}\right\|^{3}} I_{3 x 3} \\
\frac{\partial \boldsymbol{a}_{\text {SRP }}}{\partial \boldsymbol{r}}=-P_{\text {SRP }}(C)(A)\left(\frac{-I_{3 x 3}}{\left\|\rho_{\text {Sun }}-\boldsymbol{r}\right\|}+\frac{\rho_{\text {Sun }}-\boldsymbol{r}}{\left\|\rho_{\text {Sun }}-\boldsymbol{r}\right\|^{2}} \frac{\left(\rho_{\text {Sun }}-\boldsymbol{r}\right)^{T}}{\left\|\rho_{\text {Sun }}-\boldsymbol{r}\right\|} I_{3 x 3}\right) \\
\frac{\partial \dot{\boldsymbol{v}}}{\partial C}=-P_{S R P}(A)\left(\frac{\rho_{\text {Sun }}-\boldsymbol{r}}{\left\|\rho_{\text {Sun }}-\boldsymbol{r}\right\|}\right) \\
\frac{\partial \dot{\boldsymbol{v}}}{\partial A}=-P_{S R P}(C)\left(\frac{\rho_{\text {Sun }}-\boldsymbol{r}}{\left\|\rho_{\text {Sun }}-\boldsymbol{r}\right\|}\right)
\end{gathered}
$$

and the Jacobian $\hat{F}_{\hat{\boldsymbol{x}}}$ is: 


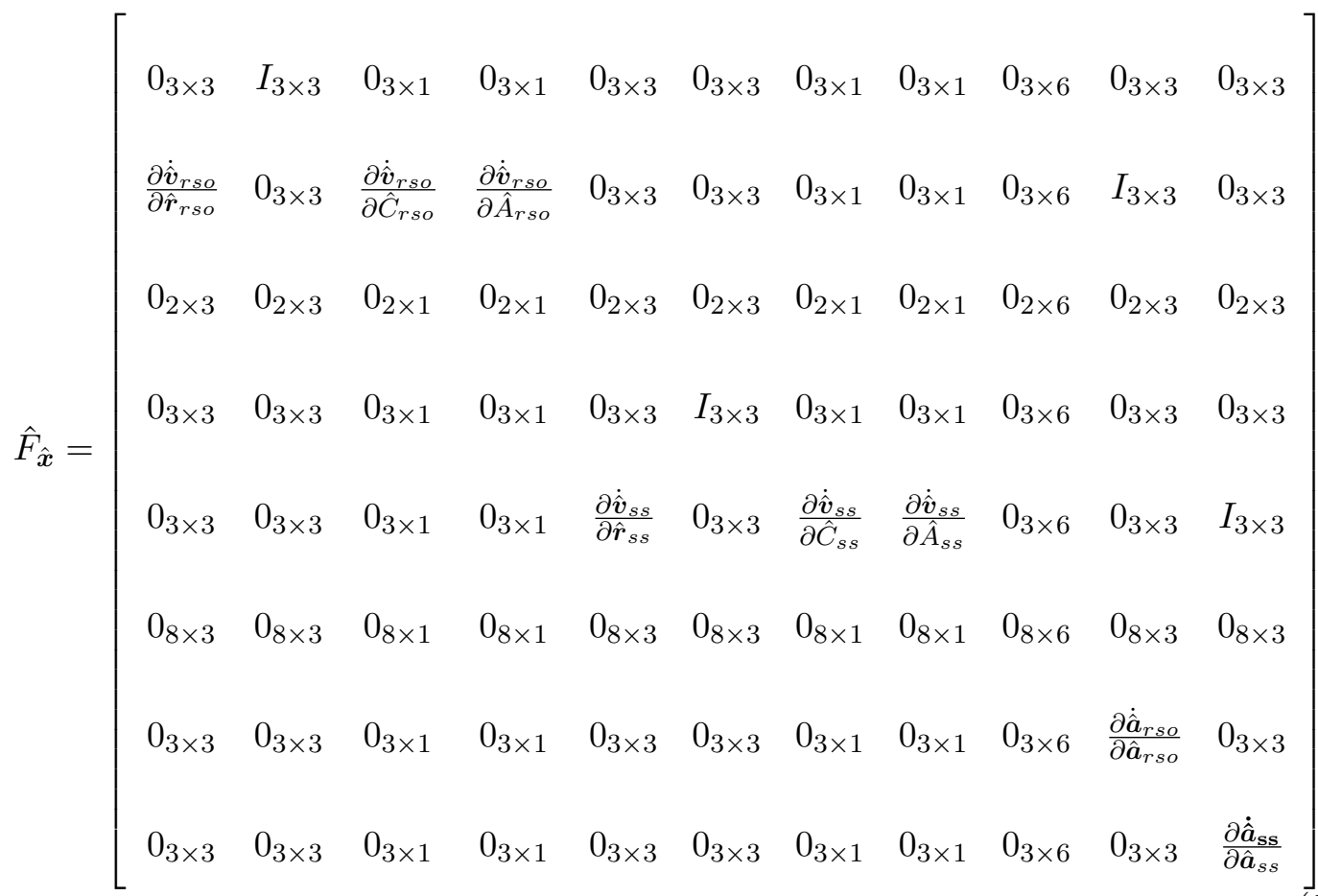

where:

$$
\begin{gathered}
\frac{\partial \dot{\hat{\boldsymbol{v}}}}{\partial \hat{C}}=-P_{S R P}(\hat{A})\left(\frac{\rho_{\text {Sun }}-\hat{\boldsymbol{r}}}{\left\|\rho_{\text {Sun }}-\hat{\boldsymbol{r}}\right\|}\right) \\
\frac{\partial \dot{\hat{\boldsymbol{v}}}}{\partial \hat{A}}=-P_{S R P}(\hat{C})\left(\frac{\rho_{\text {Sun }}-\hat{\boldsymbol{r}}}{\left\|\rho_{\text {Sun }}-\hat{\boldsymbol{r}}\right\|}\right) \\
\frac{\partial \dot{\hat{\boldsymbol{a}}}}{\partial \hat{\boldsymbol{a}}}=\frac{-1}{\tau} I_{3 x 3}
\end{gathered}
$$

and

$$
\frac{\partial \dot{\hat{\boldsymbol{v}}}}{\partial \hat{\boldsymbol{r}}}=\frac{\partial \boldsymbol{a}_{2,2}}{\partial \hat{\boldsymbol{r}}}+\frac{\partial \boldsymbol{a}_{\text {moon }}}{\partial \hat{\boldsymbol{r}}}+\frac{\partial \boldsymbol{a}_{\text {sun }}}{\partial \hat{\boldsymbol{r}}}+\frac{\partial \boldsymbol{a}_{S R P}}{\partial \hat{\boldsymbol{r}}}
$$


where

$$
\begin{gathered}
\frac{\partial \boldsymbol{a}_{2,2}}{\partial \hat{\boldsymbol{r}}}=-\frac{\mu}{\hat{r}^{3}}\left(-3 \hat{i}^{T}+I_{3 x 3}\right)-\frac{\mu(J 2)\left(R_{\oplus}^{2}\right)}{2} \\
\left\{\left(\frac{-30\left(\boldsymbol{n}^{T} \hat{\boldsymbol{r}}\right) \boldsymbol{n}\left(\hat{i}^{T}\right)}{\hat{r}^{6}}\right)+\left(\frac{6\left(\boldsymbol{n} \boldsymbol{n}^{T}\right)}{\hat{r}^{5}}\right)-\left(\frac{15 \hat{\boldsymbol{r}}\left(\hat{i}^{T}\right)}{\hat{r}^{6}}\right)+\left(\frac{3\left(I_{3 x 3}\right)}{\hat{r}^{5}}\right)+\right. \\
\left.\left(\frac{75\left(\hat{i}^{T} \boldsymbol{n}\right)^{2} \hat{\mathbf{r}}\left(\hat{i}^{T}\right)}{\hat{r}^{6}}\right)-\left(\frac{15\left(\hat{i}^{T} \boldsymbol{n}\right) I_{3 x 3}}{\hat{r}^{5}}\right)-\left(\frac{30 \hat{\boldsymbol{r}}\left(\boldsymbol{n}^{T} \hat{i}\right) \boldsymbol{n}^{T}}{\hat{r}^{6}}\right)\left(I_{3 x 3}-\hat{i} \hat{i}^{T}\right)\right\} \\
\boldsymbol{n}=\left[\begin{array}{lll}
0 & 0 & 1
\end{array}\right]^{T}
\end{gathered}
$$

Note: This partial derivative includes only $J_{2,0}$ and does not include the partial derivative for $J_{2,2}$.

$$
\begin{gathered}
\frac{\partial \boldsymbol{a}_{\text {moon }}}{\partial \hat{\boldsymbol{r}}}=\left(3 \mu_{\text {Moon }} \frac{\hat{\boldsymbol{r}}-\rho_{\text {Moon }}}{\left\|\hat{\boldsymbol{r}}-\rho_{\text {Moon }}\right\|^{4}} \frac{\left(\hat{\boldsymbol{r}}-\rho_{\text {Moon }}\right)^{T}}{\left\|\hat{\boldsymbol{r}}-\rho_{\text {Moon }}\right\|}\right)-\frac{\mu_{\text {Moon }}}{\left\|\hat{\boldsymbol{r}}-\rho_{\text {Moon }}\right\|^{3}} I_{3 x 3} \\
\frac{\partial \boldsymbol{a}_{\text {sun }}}{\partial \hat{\boldsymbol{r}}}=\left(3 \mu_{\text {Sun }} \frac{\hat{\boldsymbol{r}}-\rho_{\text {Sun }}}{\left\|\hat{\boldsymbol{r}}-\rho_{\text {Sun }}\right\|^{4}} \frac{\left(\hat{\boldsymbol{r}}-\rho_{\text {Sun }}\right)^{T}}{\left\|\hat{\boldsymbol{r}}-\rho_{\text {Sun }}\right\|}\right)-\frac{\mu_{\text {Sun }}}{\left\|\hat{\boldsymbol{r}}-\rho_{\text {Sun }}\right\|^{3}} I_{3 x 3} \\
\frac{\partial \boldsymbol{a}_{\text {SRP }}}{\partial \hat{\boldsymbol{r}}}=-P_{\text {SRP }}(\hat{C})(\hat{A})\left(\frac{-I_{3 x 3}}{\left\|\rho_{\text {Sun }}-\hat{\boldsymbol{r}}\right\|}+\frac{\rho_{\text {Sun }}-\hat{\boldsymbol{r}}}{\left\|\rho_{\text {Sun }}-\hat{\boldsymbol{r}}\right\|^{2}} \frac{\left(\rho_{\text {Sun }}-\hat{\boldsymbol{r}}\right)^{T}}{\left\|\rho_{\text {Sun }}-\hat{\boldsymbol{r}}\right\|} I_{3 x 3}\right) \\
\frac{\partial \dot{\hat{\boldsymbol{v}}}}{\partial \hat{C}}=-P_{S R P}(\hat{A})\left(\frac{\rho_{\text {Sun }}-\hat{\boldsymbol{r}}}{\left\|\rho_{\text {Sun }}-\hat{\boldsymbol{r}}\right\|}\right) \\
\frac{\partial \hat{\hat{\boldsymbol{v}}}}{\partial \hat{A}}=-P_{S R P}(\hat{C})\left(\frac{\rho_{\text {Sun }}-\hat{\boldsymbol{r}}}{\left\|\rho_{\text {Sun }}-\hat{\boldsymbol{r}}\right\|}\right)
\end{gathered}
$$

Both Jacobians, $F_{\boldsymbol{x}}$ and $\hat{F}_{\hat{\boldsymbol{x}}}$, are evaluated using the nominal trajectory.

\subsubsection{LinCov Covariance Update}

To update the LinCov covariance, as shown in Eq. 8.23 , the matrix $\mathcal{A}_{k}$ must be defined. The measurement model partial derivatives, $H_{k}$ and $\hat{H}_{k}$, are required. Both matrices consist of four separate matrices, each supporting one of the measurement sources. When a particular measurement source is used to obtain a measurement, the corresponding 
matrix to that source is used.

$$
\begin{aligned}
& H_{k, 1}= {\left[\begin{array}{cccccc}
\frac{\partial \alpha_{G S 1}}{\partial \boldsymbol{r}_{r s o}} & 0_{1 \times 5} & 0_{1 \times 3} & 0_{1 \times 5} & \frac{\partial \alpha_{G S 1}}{\partial b_{\alpha 1}} & 0_{1 \times 11} \\
\frac{\partial \delta_{G S 1}}{\partial \boldsymbol{r}_{r s o}} & 0_{1 \times 5} & 0_{1 \times 3} & 0_{1 \times 6} & \frac{\partial \delta_{G S 1}}{\partial b_{\delta 1}} & 0_{1 \times 10}
\end{array}\right] } \\
& H_{k, 2}= {\left[\begin{array}{cccccc}
\frac{\partial \alpha_{G S 2}}{\partial \boldsymbol{r}_{r s o}} & 0_{1 \times 5} & 0_{1 \times 3} & 0_{1 \times 7} & \frac{\partial \alpha_{G S 2}}{\partial b_{\alpha 2}} & 0_{1 \times 9} \\
\frac{\partial \delta_{G S 2}}{\partial \boldsymbol{r}_{r s o}} & 0_{1 \times 5} & 0_{1 \times 3} & 0_{1 \times 8} & \frac{\partial \delta_{G S 2}}{\partial b_{\delta 2}} & 0_{1 \times 8}
\end{array}\right] } \\
& H_{k, s s}= {\left[\begin{array}{cccccc}
\frac{\partial \alpha_{S S}}{\partial \boldsymbol{r}_{r s o}} & 0_{1 \times 5} & \frac{\partial \alpha_{S S}}{\partial \boldsymbol{r}_{s s}} & 0_{1 \times 9} & \frac{\partial \alpha_{S S}}{\partial b_{\alpha, s s}} & 0_{1 \times 7} \\
\frac{\partial \delta_{S S}}{\partial \boldsymbol{r}_{r s o}} & 0_{1 \times 5} & \frac{\partial \delta_{S S}}{\partial \boldsymbol{r}_{s s}} & 0_{1 \times 10} & \frac{\partial \delta_{S S}}{\partial b_{\delta, s s}} & 0_{1 \times 6}
\end{array}\right] } \\
& H_{k, g p s}=\left[\begin{array}{llllll}
0_{3 \times 8} & I_{3 \times 3} & 0_{3 \times 3} & 0_{3 \times 14} & \\
0 & & & & &
\end{array}\right]
\end{aligned}
$$

where:

$$
\begin{gathered}
\frac{\partial \alpha_{G S 1}}{\partial \boldsymbol{r}_{r s o}}=\left[\begin{array}{lll}
-\frac{\rho_{1, j}}{\left(\rho_{1, i}^{2}+\rho_{1, j}^{2}\right)} & \frac{\rho_{1, i}}{\left(\rho_{1, i}^{2}+\rho_{1, j}^{2}\right)} & 0
\end{array}\right] \\
\frac{\partial \alpha_{G S 1}}{\partial b_{\alpha 1}}=1 \\
\frac{\partial \delta_{G S 1}}{\partial \boldsymbol{r}_{r s o}}=\left[\begin{array}{cl}
-\frac{\rho_{1, i} \rho_{1, k}}{\rho_{1}^{3}\left(\sqrt{1-\frac{\rho_{1, k}^{2}}{\rho_{1}^{2}}}\right)}-\frac{\rho_{1, j} \rho_{1, k}}{\rho_{1}^{3}\left(\sqrt{1-\frac{\rho_{1, k}^{2}}{\rho_{1}^{2}}}\right)} & \frac{\rho_{1}^{2}-\rho_{1, k}^{2}}{\rho_{1}^{3}\left(\sqrt{1-\frac{\rho_{1, k}^{2}}{\rho_{1}^{2}}}\right)}
\end{array}\right] \\
\frac{\partial \alpha_{G S 2}}{\partial \boldsymbol{r}_{r s o}}=\left[\begin{array}{cl}
\frac{\partial \delta_{G S 1}}{\partial b_{\delta 1}}=1 & 0 \\
-\frac{\rho_{2, j}}{\left(\rho_{2, i}^{2}+\rho_{2, j}^{2}\right)} & \frac{\rho_{2, i}}{\left(\rho_{2, i}^{2}+\rho_{2, j}^{2}\right)}
\end{array}\right] \\
\frac{\partial \alpha_{G S 2}}{\partial b_{\alpha 2}}=1
\end{gathered}
$$




$$
\begin{gathered}
\frac{\partial \delta_{G S 2}}{\partial \boldsymbol{r}_{r s o}}=\left[\begin{array}{ccc}
-\frac{\rho_{2, i} \rho_{2, k}}{\rho_{2}^{3}\left(\sqrt{1-\frac{\rho_{2, k}^{2}}{\rho_{2}^{2}}}\right)}-\frac{\hat{\rho}_{2, j} \hat{\rho}_{2, k}}{\rho_{2}^{3}\left(\sqrt{1-\frac{\rho_{2, k}^{2}}{\rho_{2}^{2}}}\right)} \frac{\rho_{2}^{2}-\rho_{2, k}^{2}}{\rho_{2}^{3}\left(\sqrt{1-\frac{\rho_{2, k}^{2}}{\rho_{2}^{2}}}\right)}
\end{array}\right] \\
\frac{\partial \delta_{G S 2}}{\partial b_{\delta 2}}=1 \\
\frac{\partial \alpha_{S S}}{\partial \boldsymbol{r}_{r s o}}=\left[\begin{array}{lll}
-\frac{\rho_{S S, j}}{\left(\rho_{S S, i}^{2}+\rho_{S S, j}^{2}\right)} & \frac{\rho_{S S, i}}{\left(\rho_{S S, i}^{2}+\rho_{S S, j}^{2}\right)} & 0
\end{array}\right] \\
\frac{\partial \alpha_{S S}}{\partial \boldsymbol{r}_{s s}}=\left[\begin{array}{cll}
-\frac{\rho_{S S, j}}{\left(\hat{\rho}_{S S, i}^{2}+\hat{\rho}_{S S, j}^{2}\right)} & \frac{\hat{\rho}_{S S, i}}{\left(\hat{\rho}_{S S, i}^{2}+\hat{\rho}_{S S, j}^{2}\right)} & 0
\end{array}\right]\left(-I_{3 x 3}\right) \\
\frac{\partial \alpha_{S S S}}{\partial b_{\alpha, s s}}=1
\end{gathered}
$$

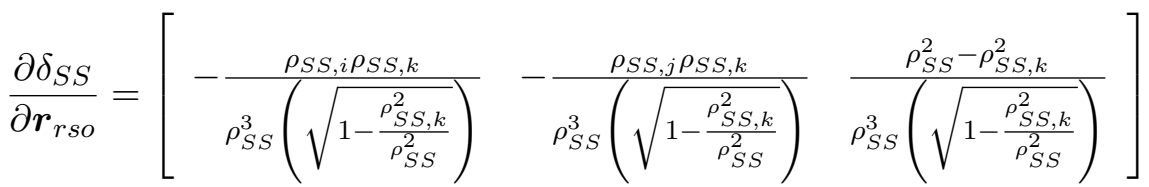$$
\frac{\partial \delta_{S S}}{\partial \boldsymbol{r}_{s S}}=\left[-\frac{\rho_{S S, i} \rho_{S S, k}}{\rho_{S S}^{3}\left(\sqrt{1-\frac{\rho_{S S, k}^{2}}{\rho_{S S}^{2}}}\right)}-\frac{\rho_{S S, j} \rho_{S S, k}}{\rho_{S S}^{3}\left(\sqrt{1-\frac{\rho_{S S, k}^{2}}{\rho_{S S}^{2}}}\right)} \frac{\rho_{S S}^{2}-\rho_{S S, k}^{2}}{\rho_{S S}^{3}\left(\sqrt{1-\frac{\rho_{S S, k}^{2}}{\rho_{S S}^{2}}}\right)}\right]\left(-I_{3 x 3}\right)
$$$$
\frac{\partial \delta_{S S}}{\partial b_{\delta, s s}}=1
$$

where $\rho_{1} \equiv\left\|\overline{\mathbf{r}}_{r s o}-\overline{\mathbf{r}}_{G S 1}\right\|, \rho_{2} \equiv\left\|\overline{\mathbf{r}}_{r s o}-\overline{\mathbf{r}}_{G S 2}\right\|, \rho_{s s} \equiv\left\|\overline{\mathbf{r}}_{r s o}-\overline{\mathbf{r}}_{s s}\right\|$ (all in inertial frame) and $\rho_{1, i / j / k}, \rho_{2, i / j / k}, \rho_{s s, i / j / k}$ are the inertial components.

$$
\begin{aligned}
\hat{H}_{k, 1}= & {\left[\begin{array}{cccccc}
\frac{\partial \alpha_{G S 1}}{\partial \hat{r}_{r s o}} & 0_{1 \times 5} & 0_{1 \times 3} & 0_{1 \times 5} & \frac{\partial \alpha_{G S 1}}{\partial \hat{b}_{\alpha 1}} & 0_{1 \times 11} \\
\frac{\partial \delta_{G S 1}}{\partial \hat{\boldsymbol{r}}_{r s o}} & 0_{1 \times 5} & 0_{1 \times 3} & 0_{1 \times 6} & \frac{\partial \delta_{G S 1}}{\partial \hat{b}_{\delta 1}} & 0_{1 \times 10}
\end{array}\right] } \\
\hat{H}_{k, 2}= & {\left[\begin{array}{cccccc}
\frac{\partial \alpha_{G S 2}}{\partial \hat{\boldsymbol{r}}_{r s o}} & 0_{1 \times 5} & 0_{1 \times 3} & 0_{1 \times 7} & \frac{\partial \alpha_{G S 2}}{\partial \hat{b}_{\alpha 2}} & 0_{1 \times 9} \\
\frac{\partial \delta_{G S 2}}{\partial \hat{\boldsymbol{r}}_{r s o}} & 0_{1 \times 5} & 0_{1 \times 3} & 0_{1 \times 8} & \frac{\partial \delta_{G S 2}}{\partial_{\hat{b}_{\delta 2}}} & 0_{1 \times 8}
\end{array}\right] }
\end{aligned}
$$




$$
\begin{gathered}
\hat{H}_{k, s s}=\left[\begin{array}{cccccc}
\frac{\partial \alpha_{S S}}{\partial \hat{\boldsymbol{r}}_{r s o}} & 0_{1 \times 5} & \frac{\partial \alpha_{S S}}{\partial \hat{\boldsymbol{r}}_{s s}} & 0_{1 \times 9} & \frac{\partial \alpha_{S S}}{\partial \hat{b}_{\alpha, s s}} & 0_{1 \times 7} \\
\frac{\partial \delta_{S S}}{\partial \hat{\boldsymbol{r}}_{r s o}} & 0_{1 \times 5} & \frac{\partial \delta_{S S}}{\partial \hat{\boldsymbol{r}}_{s s}} & 0_{1 \times 10} & \frac{\partial \delta_{S S}}{\partial \hat{b}_{\delta, s s}} & 0_{1 \times 6}
\end{array}\right] \\
\hat{H}_{k, g p s}=\left[\begin{array}{cccc}
0_{3 \times 8} & I_{3 \times 3} & 0_{3 \times 3} & 0_{3 \times 14} \\
0_{3 \times 8} & 0_{3 \times 3} & I_{3 \times 3} & 0_{3 \times 14}
\end{array}\right]
\end{gathered}
$$

where:

$$
\begin{aligned}
& \frac{\partial \alpha_{G S 1}}{\partial \hat{\boldsymbol{r}}_{r s o}}=\left[\begin{array}{lll}
-\frac{\hat{\rho}_{1, j}}{\left(\hat{\rho}_{1, i}^{2}+\hat{\rho}_{1, j}^{2}\right)} & \frac{\hat{\rho}_{1, i}}{\left(\hat{\rho}_{1, i}^{2}+\hat{\rho}_{1, j}^{2}\right)} & 0
\end{array}\right] \\
& \frac{\partial \alpha_{G S 1}}{\partial \hat{b}_{\alpha 1}}=1
\end{aligned}
$$

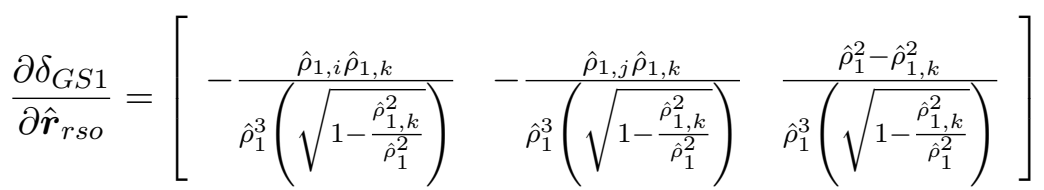

$$
\begin{aligned}
& \frac{\partial \delta_{G S 1}}{\partial \hat{b}_{\delta 1}}=1 \\
& \frac{\partial \alpha_{G S 2}}{\partial \hat{\boldsymbol{r}}_{r s o}}=\left[\begin{array}{lll}
-\frac{\hat{\rho}_{2, j}}{\left(\hat{\rho}_{2, i}^{2}+\hat{\rho}_{2, j}^{2}\right)} & \frac{\hat{\rho}_{2, i}}{\left(\hat{\rho}_{2, i}^{2}+\hat{\rho}_{2, j}^{2}\right)} & 0
\end{array}\right] \\
& \frac{\partial \alpha_{G S 2}}{\partial \hat{b}_{\alpha 2}}=1
\end{aligned}
$$

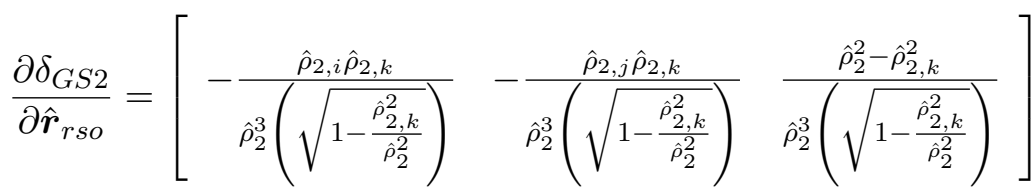

$$
\begin{aligned}
& \frac{\partial \delta_{G S 2}}{\partial \hat{b}_{\delta 2}}=1 \\
& \frac{\partial \alpha_{S S}}{\partial \hat{\boldsymbol{r}}_{r s o}}=\left[\begin{array}{lll}
-\frac{\hat{\rho}_{S S, j}}{\left(\hat{\rho}_{S S, i}^{2}+\hat{\rho}_{S S, j}^{2}\right)} & \frac{\hat{\rho}_{S S, i}}{\left(\hat{\rho}_{S S, i}^{2}+\hat{\rho}_{S S, j}^{2}\right)} & 0
\end{array}\right]
\end{aligned}
$$




$$
\begin{aligned}
& \frac{\partial \alpha_{S S}}{\partial \hat{\boldsymbol{r}}_{s s}}=\left[\begin{array}{lll}
-\frac{\hat{\rho}_{S S, j}}{\left(\hat{\rho}_{S S, i}^{2}+\hat{\rho}_{S S, j}^{2}\right)} & \frac{\hat{\rho}_{S S, i}}{\left(\hat{\rho}_{S S, i}^{2}+\hat{\rho}_{S S, j}^{2}\right)} & 0
\end{array}\right]\left(-I_{3 x 3}\right) \\
& \frac{\partial \alpha_{S S}}{\partial \hat{b}_{\alpha, s s}}=1
\end{aligned}
$$

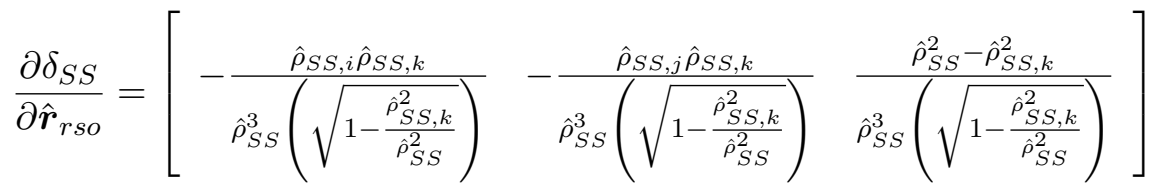

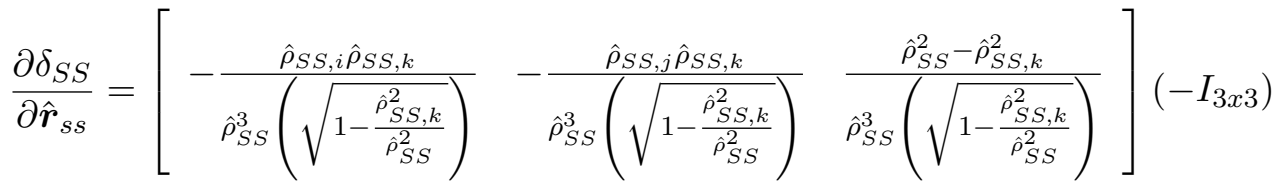

$$
\begin{aligned}
& \frac{\partial \delta_{S S}}{\partial \hat{b}_{\delta, s s}}=1
\end{aligned}
$$

where $\hat{\rho}_{1} \equiv\left\|\overline{\mathbf{r}}_{r s o}-\overline{\mathbf{r}}_{G S 1}\right\|, \hat{\rho}_{2} \equiv\left\|\overline{\mathbf{r}}_{r s o}-\overline{\mathbf{r}}_{G S 2}\right\|, \hat{\rho}_{s s} \equiv\left\|\overline{\mathbf{r}}_{r s o}-\overline{\mathbf{r}}_{s s}\right\|$ (all in inertial frame) and $\hat{\rho}_{1, i / j / k}, \hat{\rho}_{2, i / j / k}, \hat{\rho}_{s s, i / j / k}$ are the inertial components.

Both measurement partial derivative matrices, $H_{k}$ and $\hat{H}_{k}$, are evaluated using the nominal trajectory.

\subsection{Error Budget Analysis}

The developed LinCov analysis tool can be used to compute the error budgets for the state dispersions. For this, the total state dispersion is broken down into the contributions from each error source. The LinCov analysis tool is run with each true error source turned on one by one, while the rest of the true error sources remain at zero. The filter model always remains unchanged. If the error sources are uncorrelated, as is the case with this analysis, the total state dispersion is equal to the root sum square (RSS) of each individual error source. The error sources for this error budget analysis are broken down into the following 11 groups:

1) RSO initial position and velocity - This set of error sources consists of the initial error placed on the RSO position and RSO velocity. 
2) SS initial position and velocity - This set of error sources consists of the initial error placed on the space sensor position and space sensor velocity.

3) RSO area-to-mass ratio - This set of error sources consists of the initial error placed on the RSO area-to-mass ratio as well as the random walk noise, $Q_{A_{\text {rso }}}$.

4) SS area-to-mass ratio - This set of error sources consists of the initial error placed on the space sensor area-to-mass ratio as well as the random walk noise, $Q_{A_{s s}}$.

5) RSO disturbance accelerations - This consists of the $\mathrm{x}, \mathrm{y}$ and $\mathrm{z}$ components of the RSO disturbance accelerations.

6) SS disturbance accelerations - This consists of the $\mathrm{x}, \mathrm{y}$ and $\mathrm{z}$ components of the space sensor disturbance accelerations.

7) RSO process noise

8) SS process noise

9) GPS/USN errors

10) Ground station $(1 \& 2)$ errors - This set of error sources consists of the ground station measurement biases and noise.

11) SS errors - This consists of the space sensor measurement bias and noise.

\subsection{Summary}

This chapter covered the dynamics, equations and development of the LinCov analysis tool that is used for the analysis and results portion of this research. It also discussed how the developed LinCov analysis tool can be used to perform error budget analysis. 
CHAPTER 9

\section{LINEAR COVARIANCE ANALYSIS TOOL VALIDATION}

\subsection{LinCov Validation Method Overview}

The LinCov analysis tool developed in Chapter 8 must be validated prior to its use for analysis. To validate the LinCov analysis tool, the same set of verification runs used to validate the EKF are applied to the LinCov tool. The LinCov results are compared to the Monte Carlo results from Chapter 7. If the LinCov results fall within the Monte Carlo confidence intervals, the LinCov tool is considered validated. This chapter is broken into sections similar to that of Chapter 7.

\subsection{Measurement Bias Estimation}

The purpose of this analysis is to verify that LinCov estimates the measurement biases of both ground stations and the space sensor. The only error sources turned on for this analysis are the biases on each measurement, set to the nominal error source level. The low circular orbit space sensor is used for this analysis and the space sensor accuracy is set to its most accurate level. Figures 9.1 through 9.3 show that LinCov is capable of estimating the bias variance of each measurement source. The LinCov results fall within the Monte Carlo confidence intervals. 

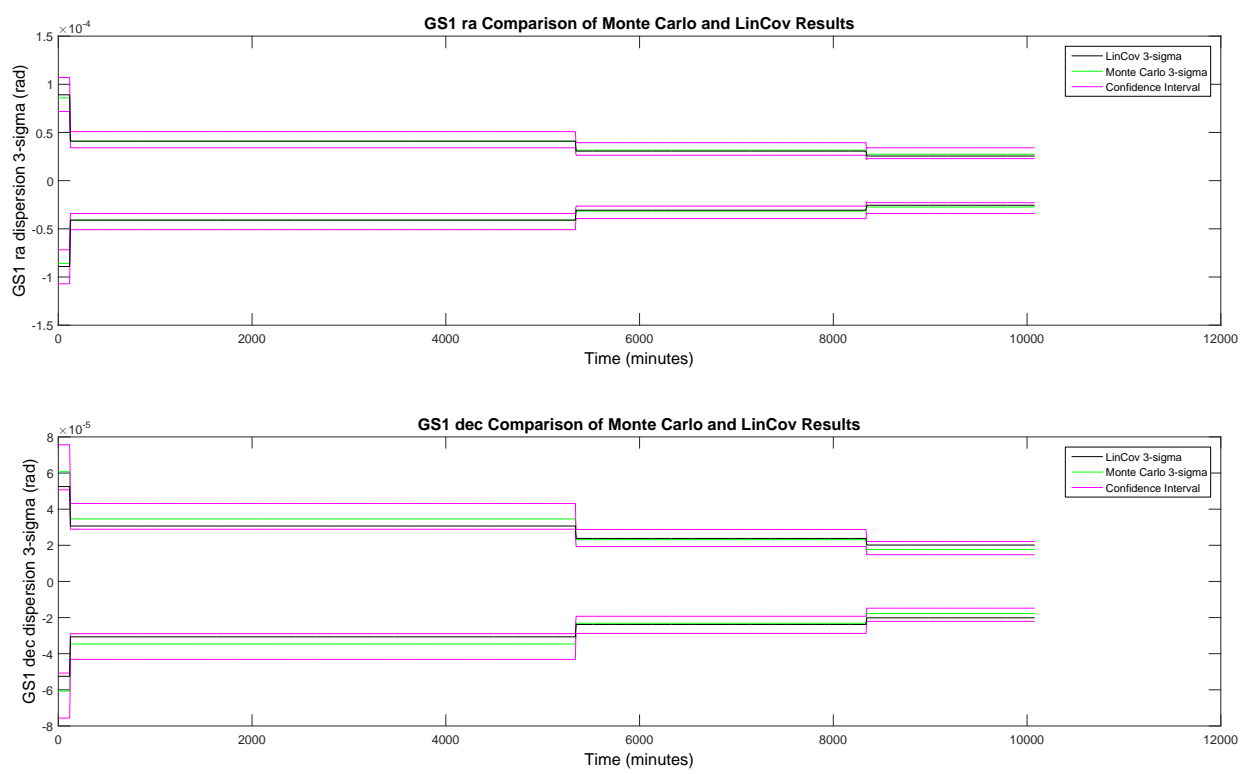

Fig. 9.1: GS1 Measurement Bias, LinCov Validation, Estimation Errors
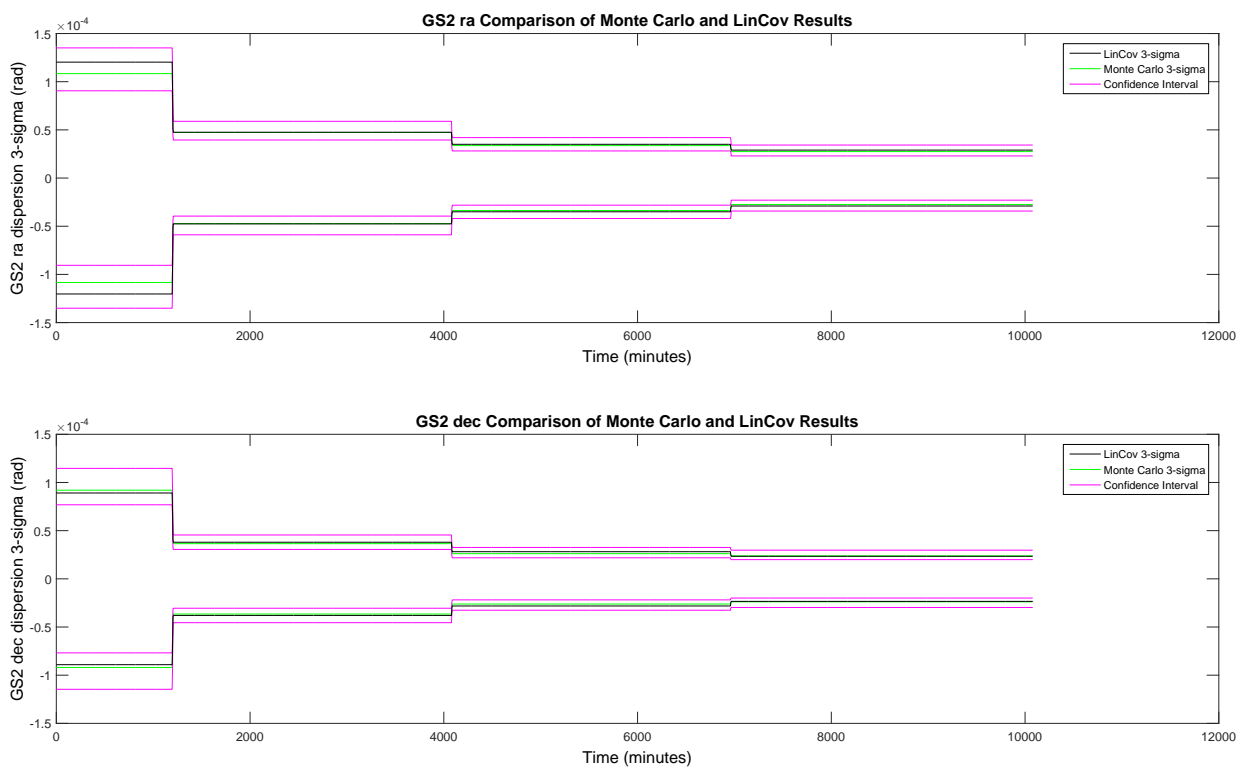

Fig. 9.2: GS2 Measurement Bias, LinCov Validation, Estimation Errors 

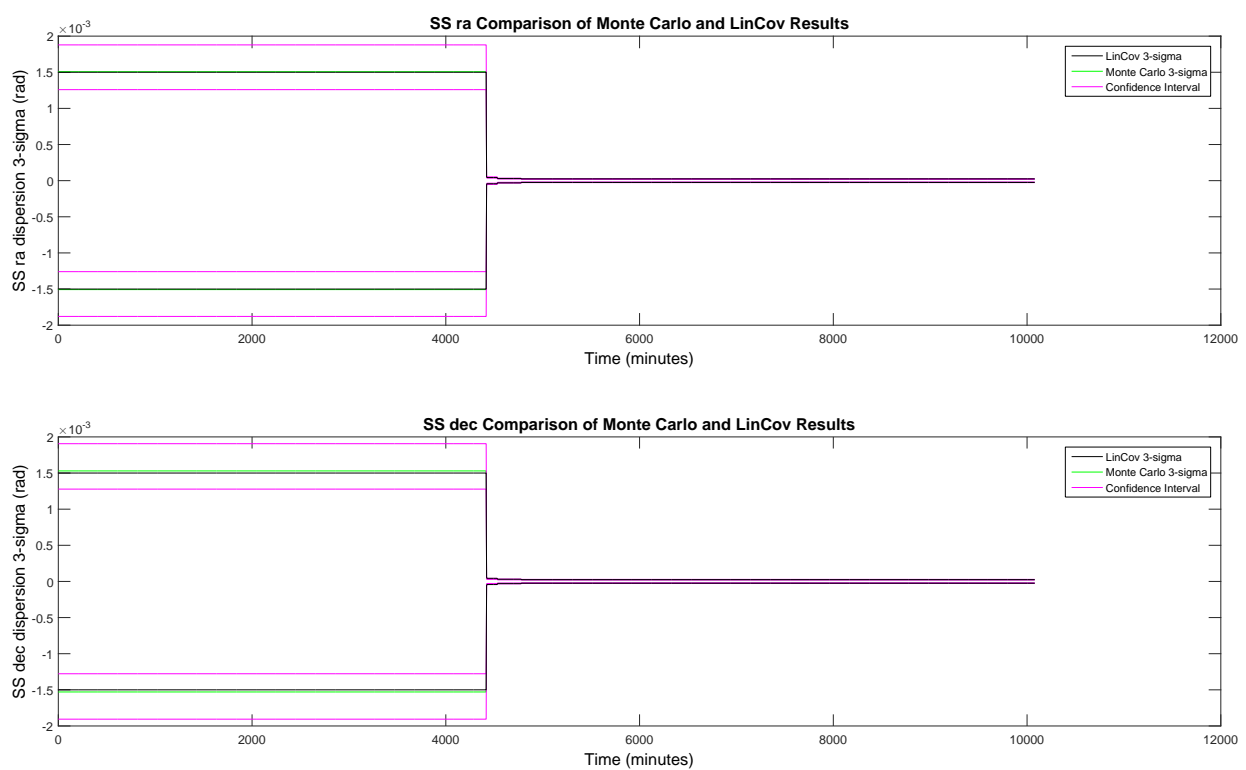

Fig. 9.3: Space Sensor Measurement Bias, LinCov Validation, Estimation Errors

\subsection{SRP Estimation}

The purpose of this analysis is to verify that LinCov estimates the solar radiation pressure of both the RSO and the space sensor. The RSO area-to-mass ratio is set to its highest initial condition error level, and the strength of the random walk noise, $Q_{A_{\text {rso }}}$, is set to its lowest error level. This simulates a large uncertainty in the value of the area-to-mass ratio, but a fairly constant value over time. The space sensor area-to-mass ratio is set to its nominal initial condition error level and nominal random walk noise. GPS updates of the space sensor are turned on in this scenario, updating the space sensor position and velocity every 10 minutes. The low circular space sensor orbit is used for this analysis and the space sensor accuracy is set to its most accurate level. Ground station measurement noise is turned on. Figures 9.4 and 9.5 show that LinCov results fall within the Monte Carlo confidence intervals. 


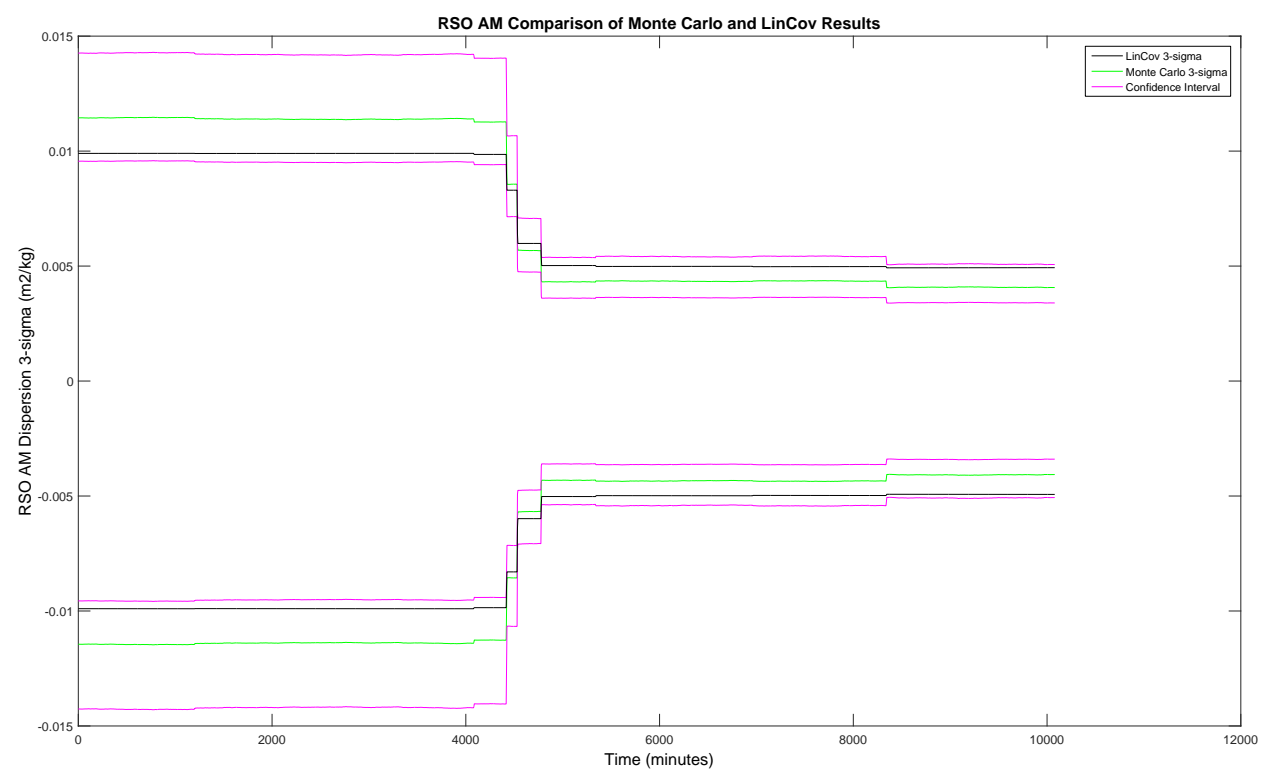

Fig. 9.4: RSO Area-to-Mass Ratio, LinCov Validation, Estimation Errors

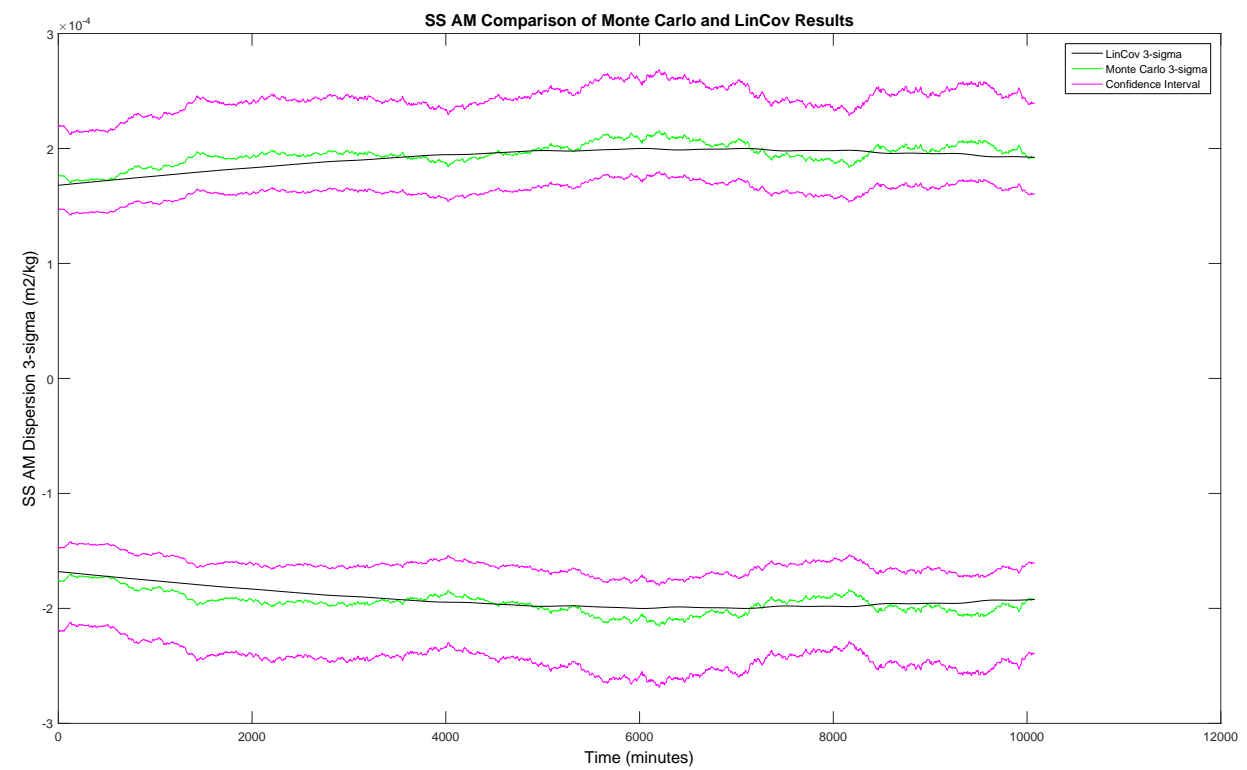

Fig. 9.5: Space Sensor Area-to-Mass Ratio, LinCov Validation, Estimation Errors 


\subsection{Disturbance Acceleration Estimation}

This analysis investigates the ability of LinCov to estimate the disturbance acceleration terms of the RSO and SS.

Figure 9.6 shows the RSO disturbance acceleration. Space sensor and ground measurement accuracies are set to the nominal level. LinCov results fall within the Monte Carlo confidence intervals.
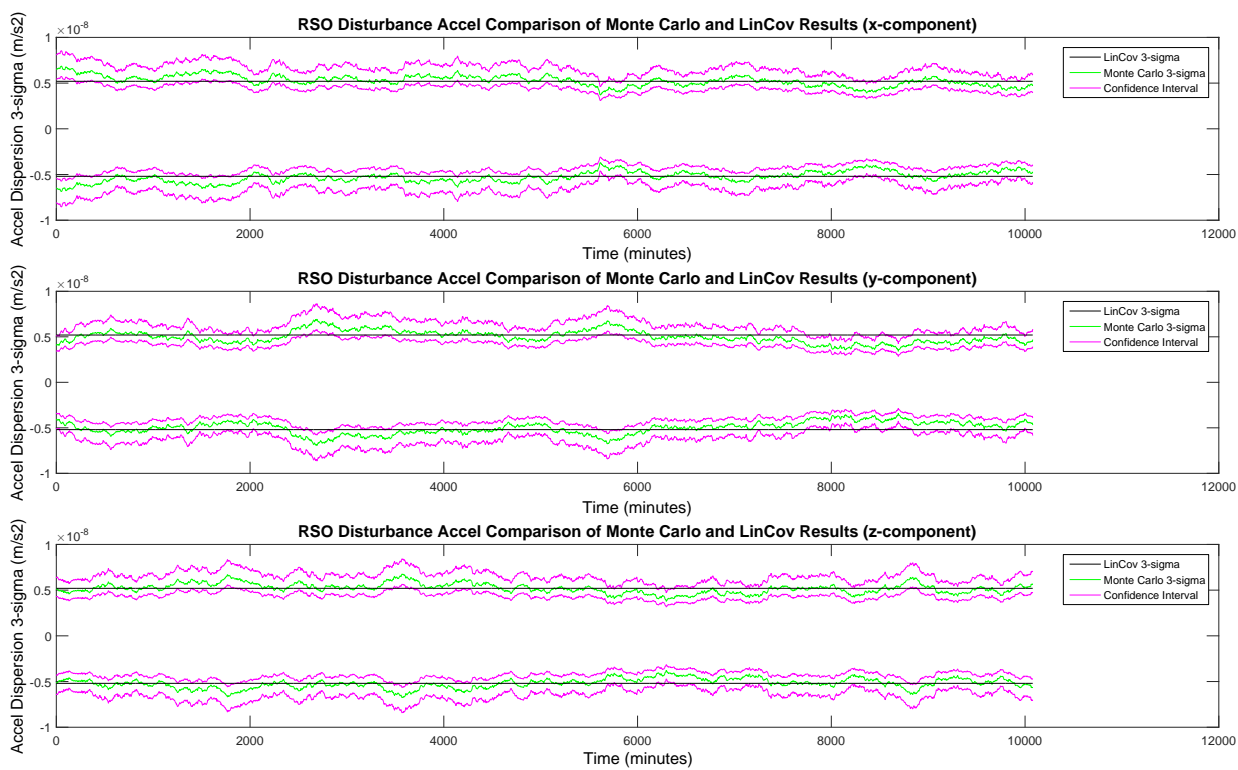

Fig. 9.6: RSO Disturbance Acceleration, LinCov Validation, Estimation Errors

Figure 9.7 shows the low circular orbit space sensor disturbance acceleration. Space sensor and ground measurement accuracies are set to the nominal level. LinCov results fall within the Monte Carlo confidence intervals. The results for the space sensor high circular orbit and the space sensor elliptical orbit were similar and all results fall within the Monte Carlo confidence intervals. 

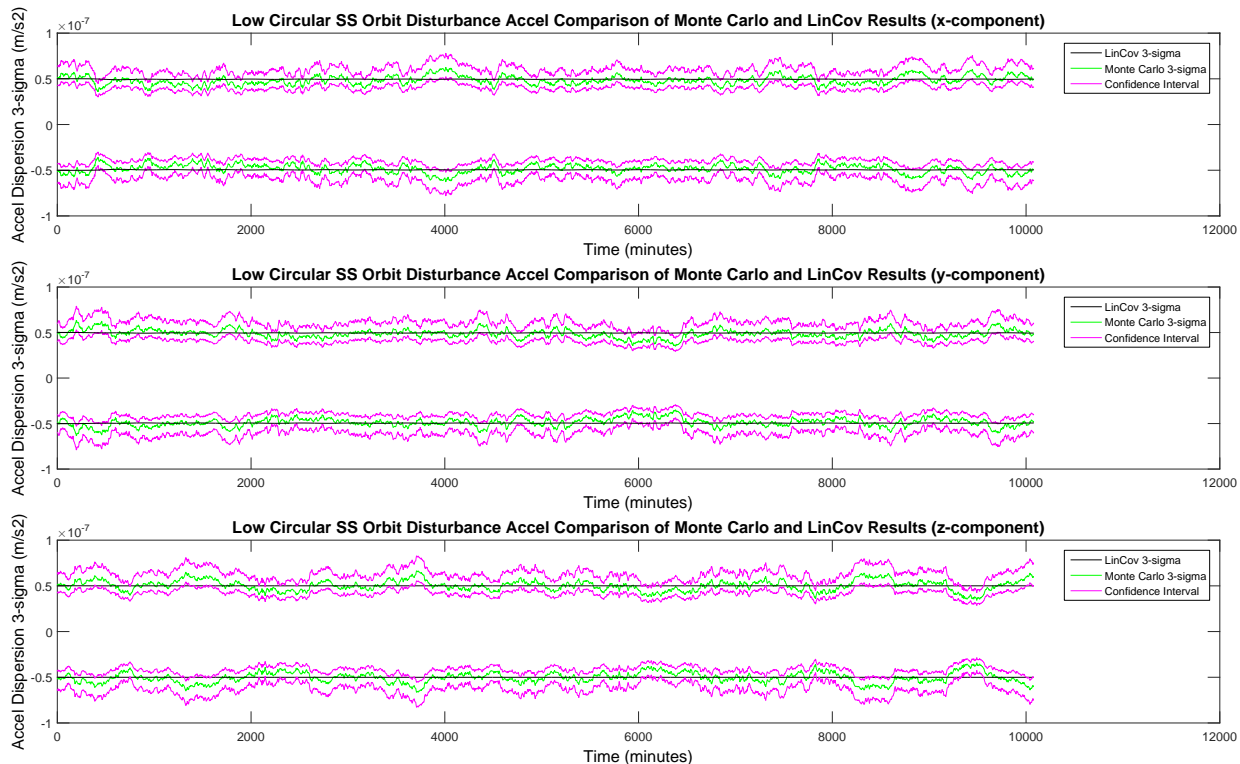

Fig. 9.7: Low Circular Space Sensor Orbit Disturbance Acceleration, LinCov Validation, Estimation Errors

\subsection{Ground Station Only Measurement Validation}

The following analysis incorporates ground station measurements of the RSO at the nominal and high error source levels. A $2 \times 2$ gravity model is used in the truth model and LinCov.

Figures 9.8-9.12 are results of ground station measurements of the RSO. All errors sources and measurement uncertainties are set to the nominal level. LinCov results are shown to stay within the Monte Carlo confidence intervals. The Monte Carlo/LinCov results for the Ground Station 2 bias were nearly identical to Ground Station 1 results. 

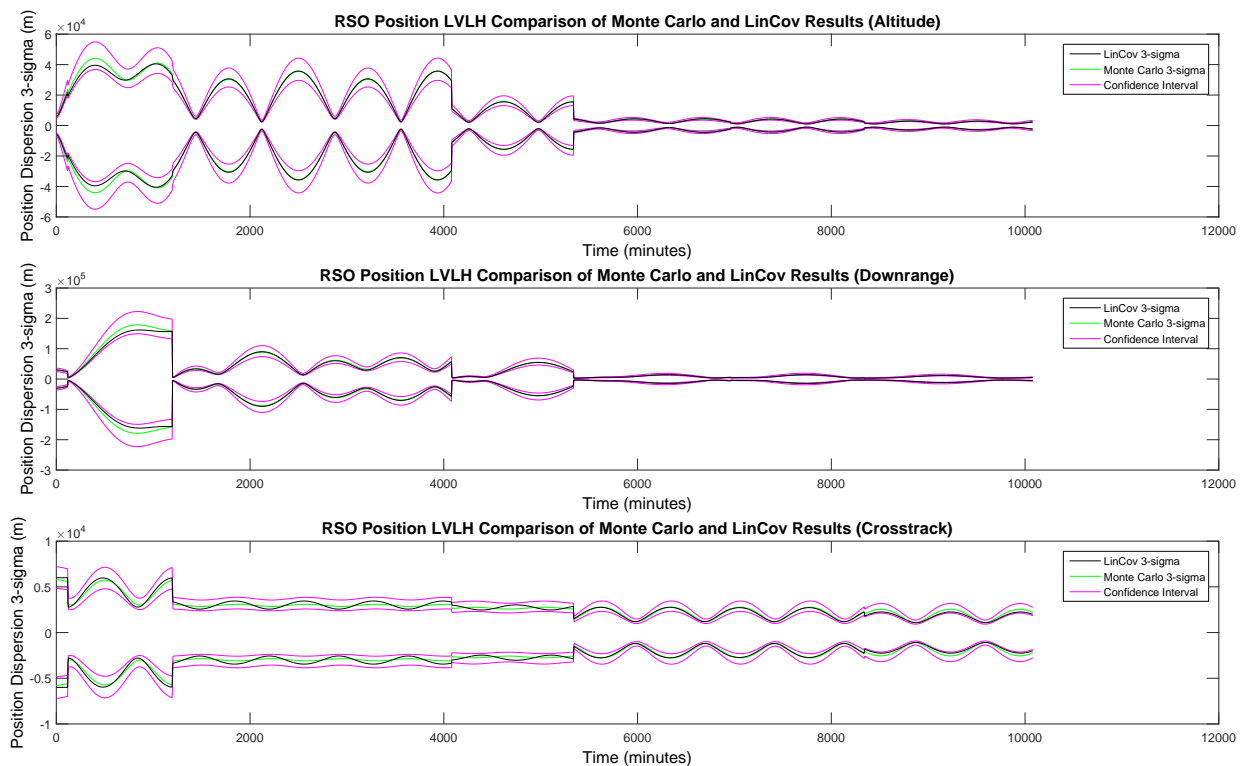

Fig. 9.8: Nominal Initial Errors - Ground Station Only Measurements, LinCov Validation, RSO LVLH Position Estimation Errors
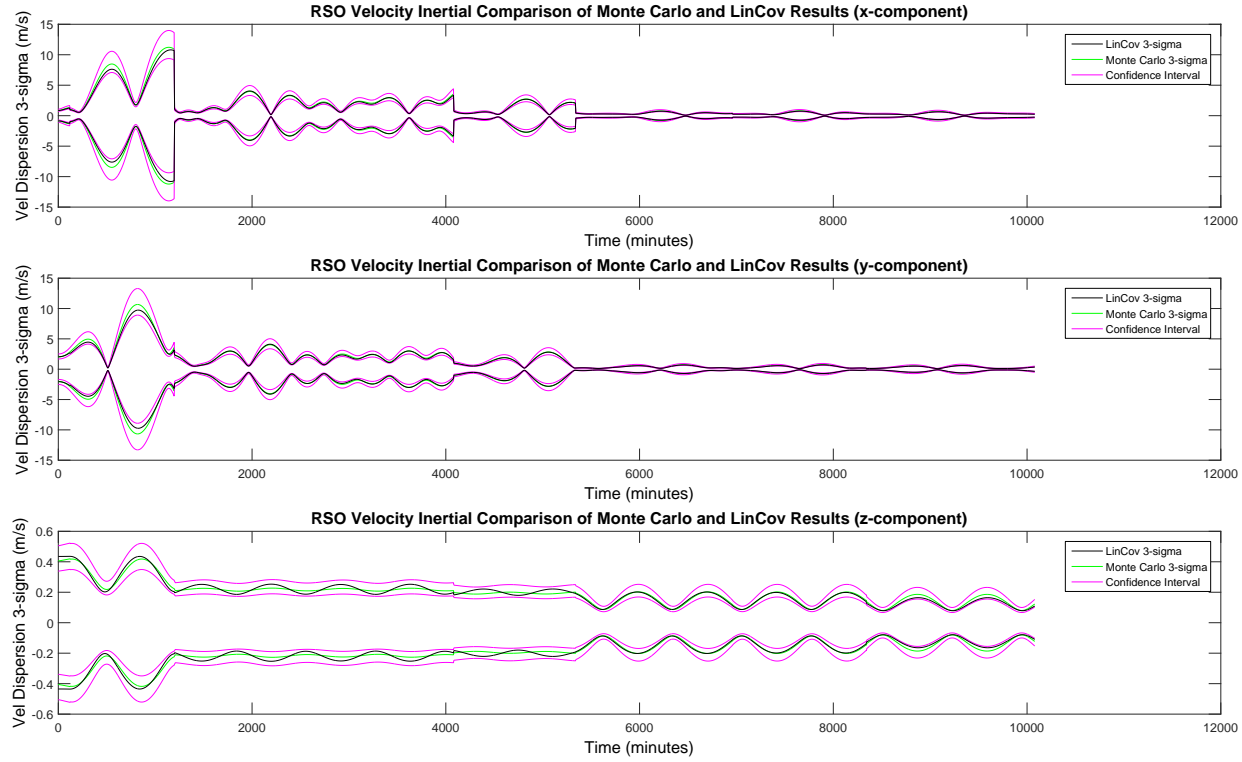

Fig. 9.9: Nominal Initial Errors - Ground Station Only Measurements, LinCov Validation, RSO Velocity Estimation Errors 


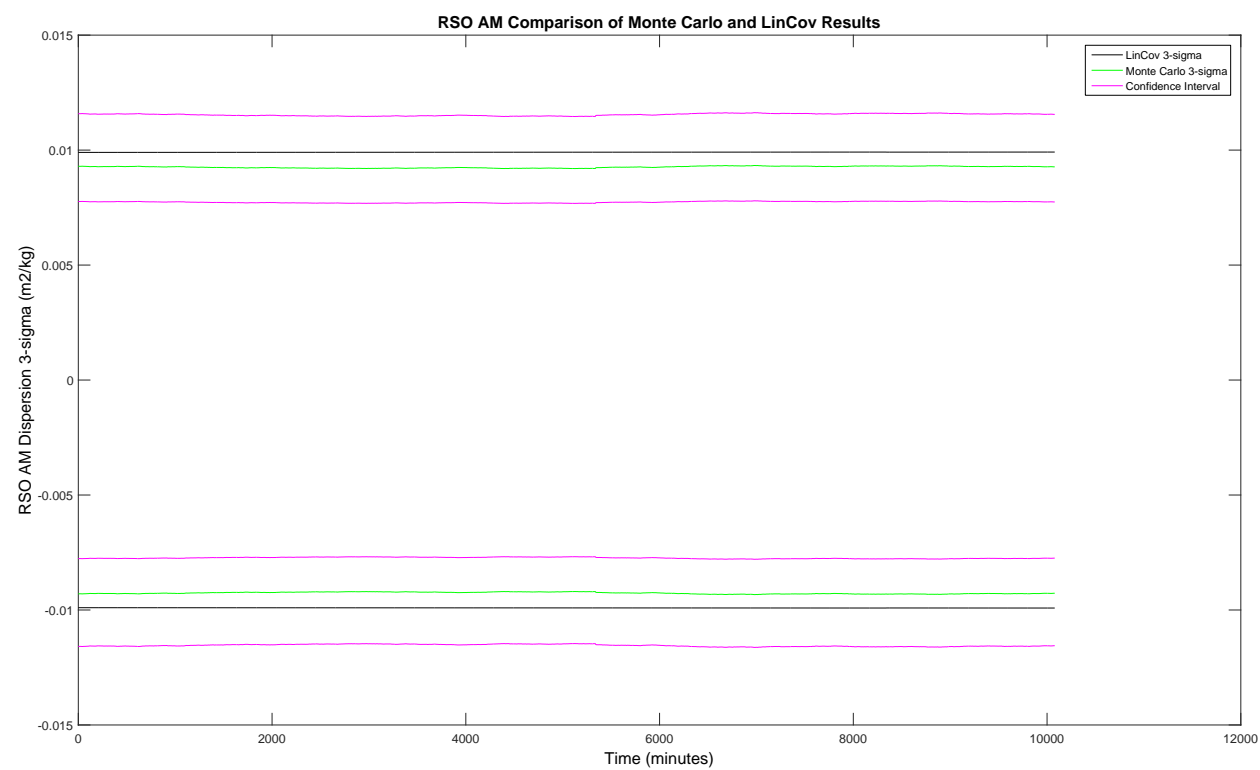

Fig. 9.10: Nominal Initial Errors - Ground Station Only Measurements, LinCov Validation, RSO Area-to-Mass Ratio Estimation Error
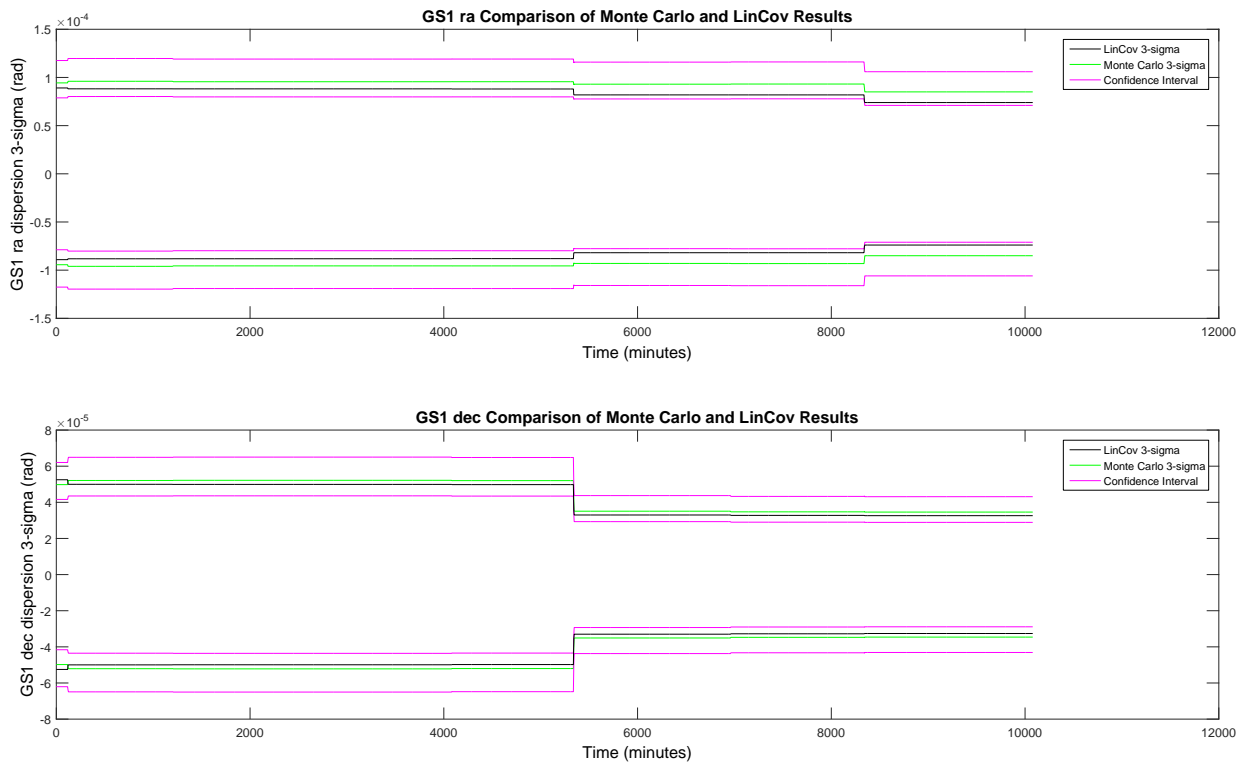

Fig. 9.11: Nominal Initial Errors - Ground Station Only Measurements, LinCov Validation, Ground Station 1 Biases Estimation Errors 

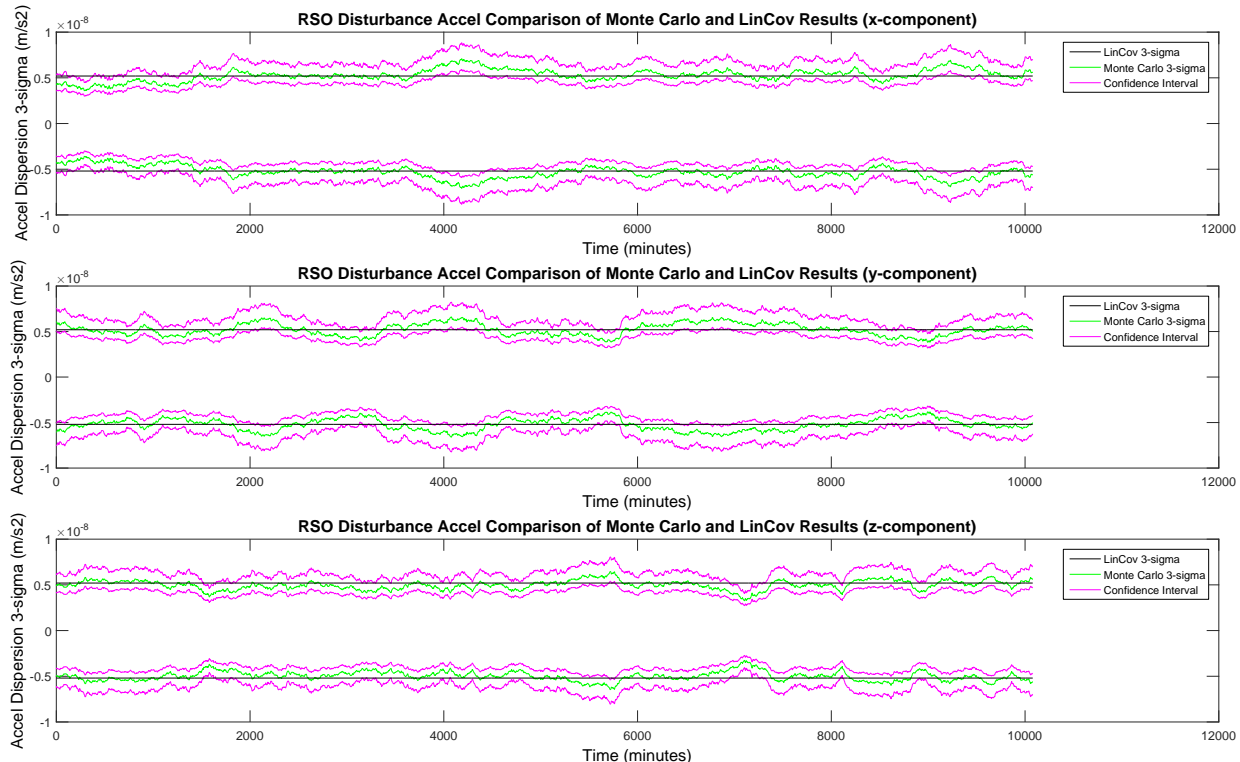

Fig. 9.12: Nominal Initial Errors - Ground Station Only Measurements, LinCov Validation, RSO Disturbance Accels Estimation Errors

Although the magnitudes of the Monte Carlo/LinCov results are larger than the nominal error cases, all results for the high level error case have similar characteristics as the low error case. The one exception is the area-to-mass ratio shown in Figure 9.13. 


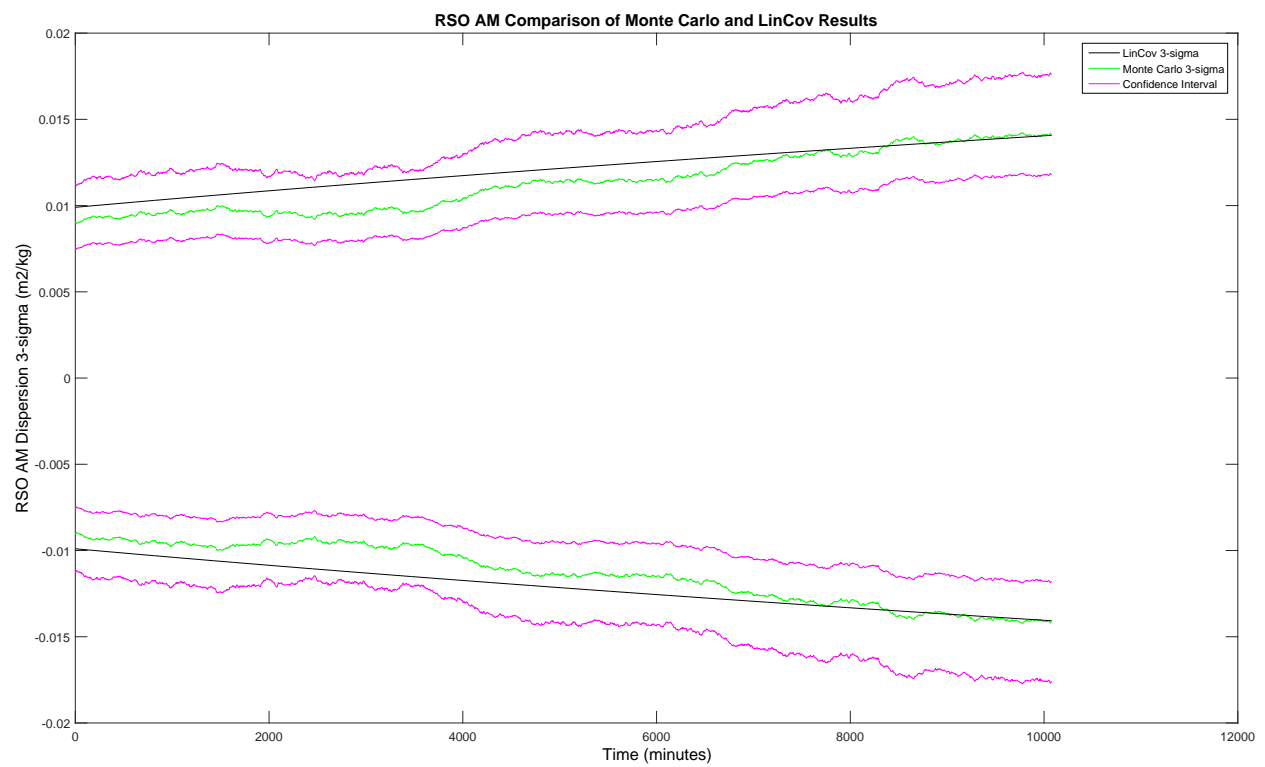

Fig. 9.13: High Initial Errors - Ground Station Only Measurements, LinCov Validation, RSO Area-to-Mass Ratio Estimation Error

\subsection{Space Sensor and Ground Station Measurement Validation}

The following analysis incorporates both space sensor and ground station measurements of the RSO. Each space sensor orbit: low circular, high circular, and elliptical, is analyzed for nominal and high error source levels. The space sensor position and velocity is updated through the use of GPS. A $2 \times 2$ gravity model is used. All relevant plots are shown for the low circular nominal error source analysis. The remaining analysis results will only show plots for the research critical results, the RSO position error in LVLH. All results met the condition of the LinCov results being within the Monte Carlo confidence intervals.

\subsubsection{Low Circular Space Sensor Orbit}

Figures 9.14-9.24 are results from a low circular orbit space sensor and ground station measurements. All error sources are set to the nominal level. LinCov results stay within the Monte Carlo confidence intervals, other than the occasional outliers. 

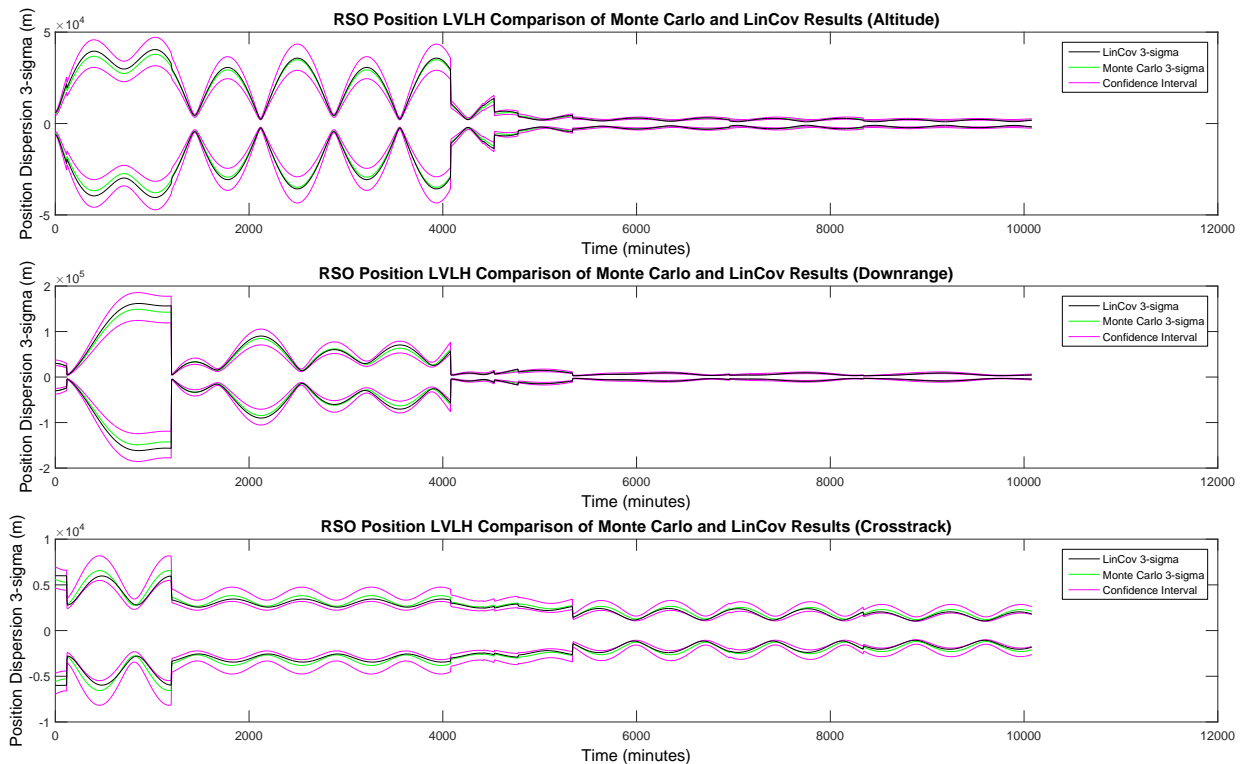

Fig. 9.14: Nominal Initial Errors - Ground Station and Low Circular SS Measurements, LinCov Validation, RSO LVLH Position Estimation Errors
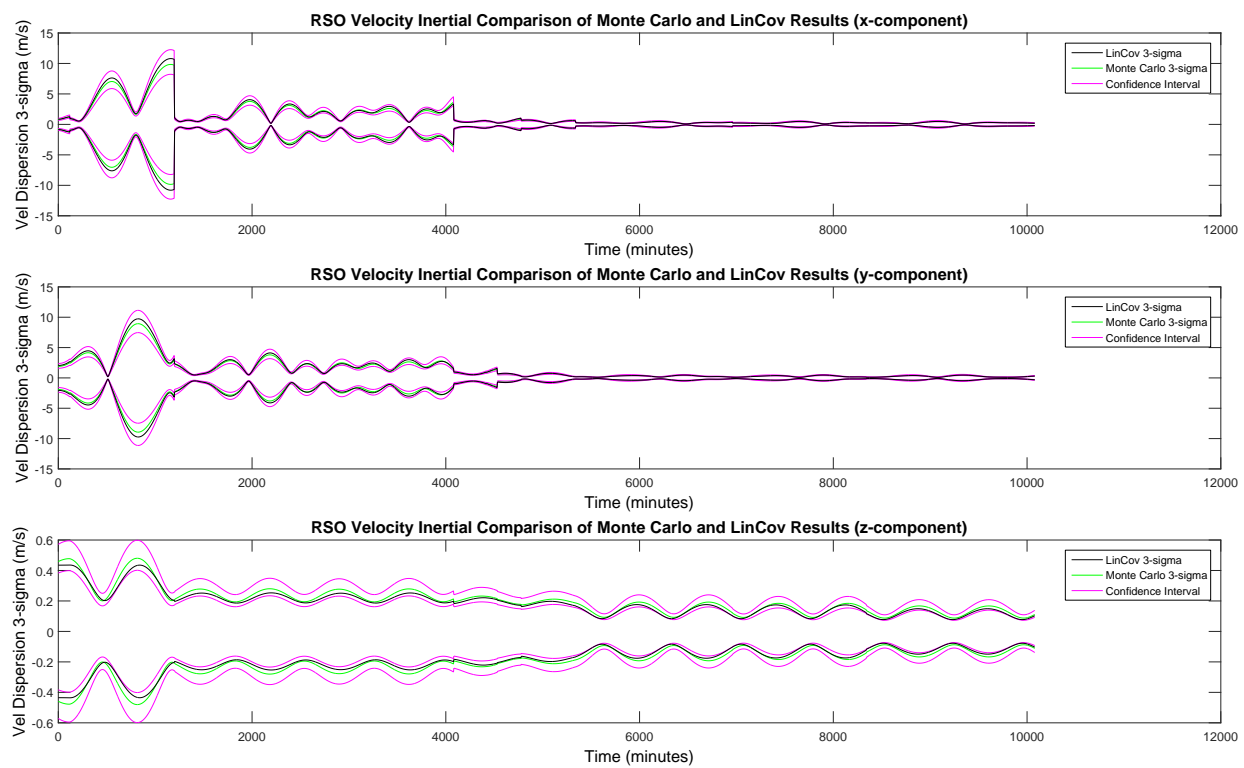

Fig. 9.15: Nominal Initial Errors - Ground Station and Low Circular SS Measurements, LinCov Validation, RSO Inertial Velocity Estimation Errors 


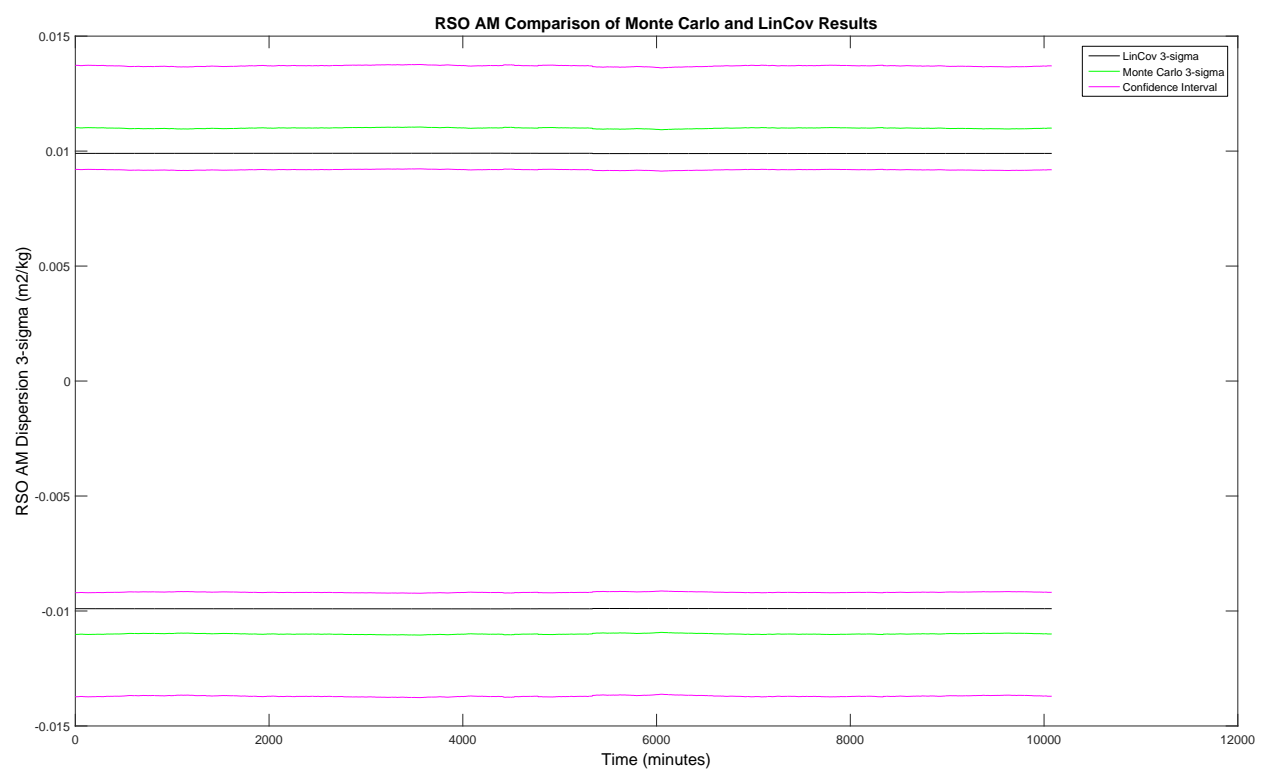

Fig. 9.16: Nominal Initial Errors - Ground Station and Low Circular SS Measurements, LinCov Validation, RSO Area to Mass Ratio Estimation Error
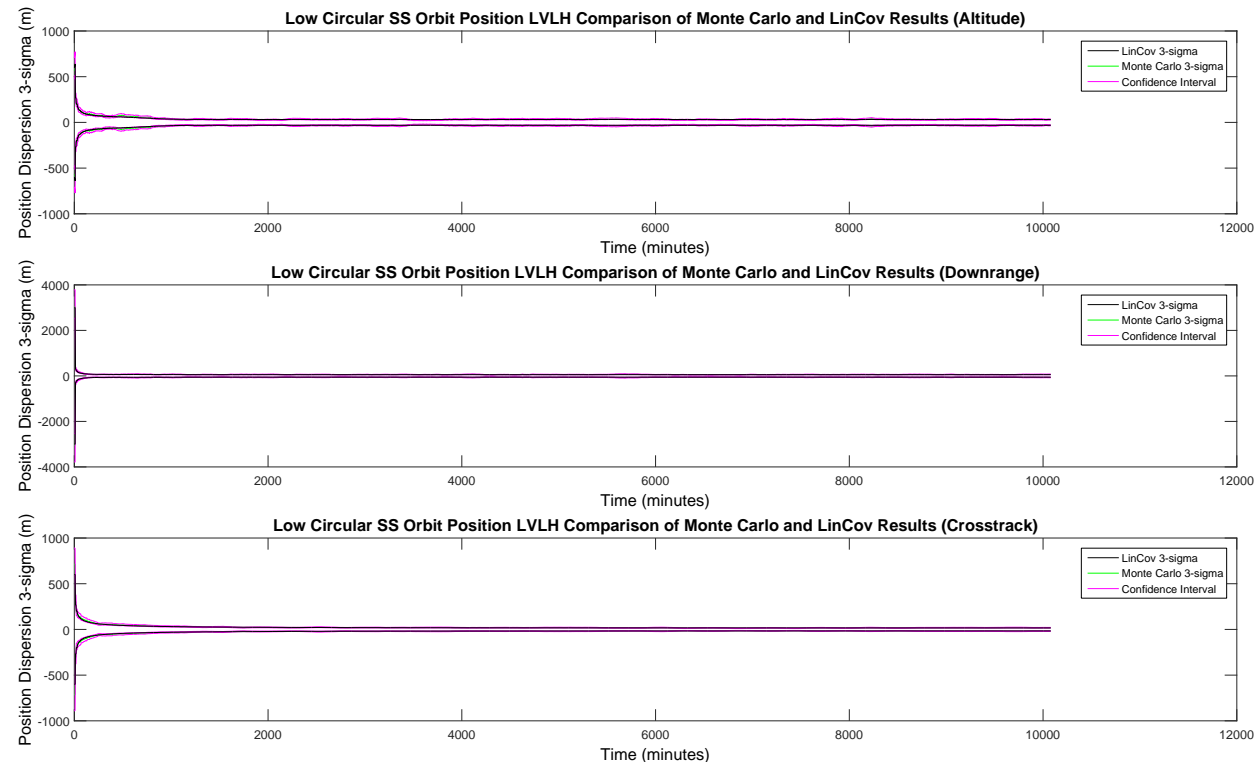

Fig. 9.17: Nominal Initial Errors - Ground Station and Low Circular SS Measurements, LinCov Validation, SS LVLH Position Estimation Errors 

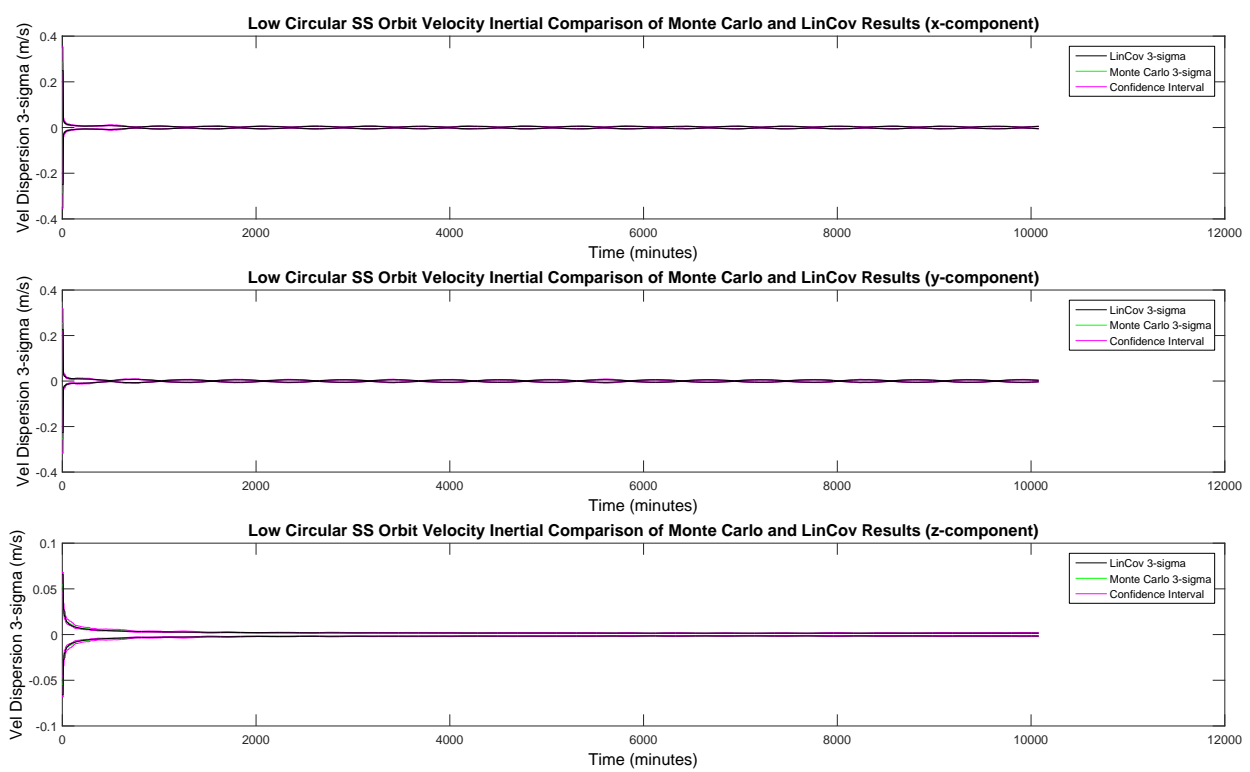

Fig. 9.18: Nominal Initial Errors - Ground Station and Low Circular SS Measurements, LinCov Validation, SS Inertial Velocity Estimation Errors

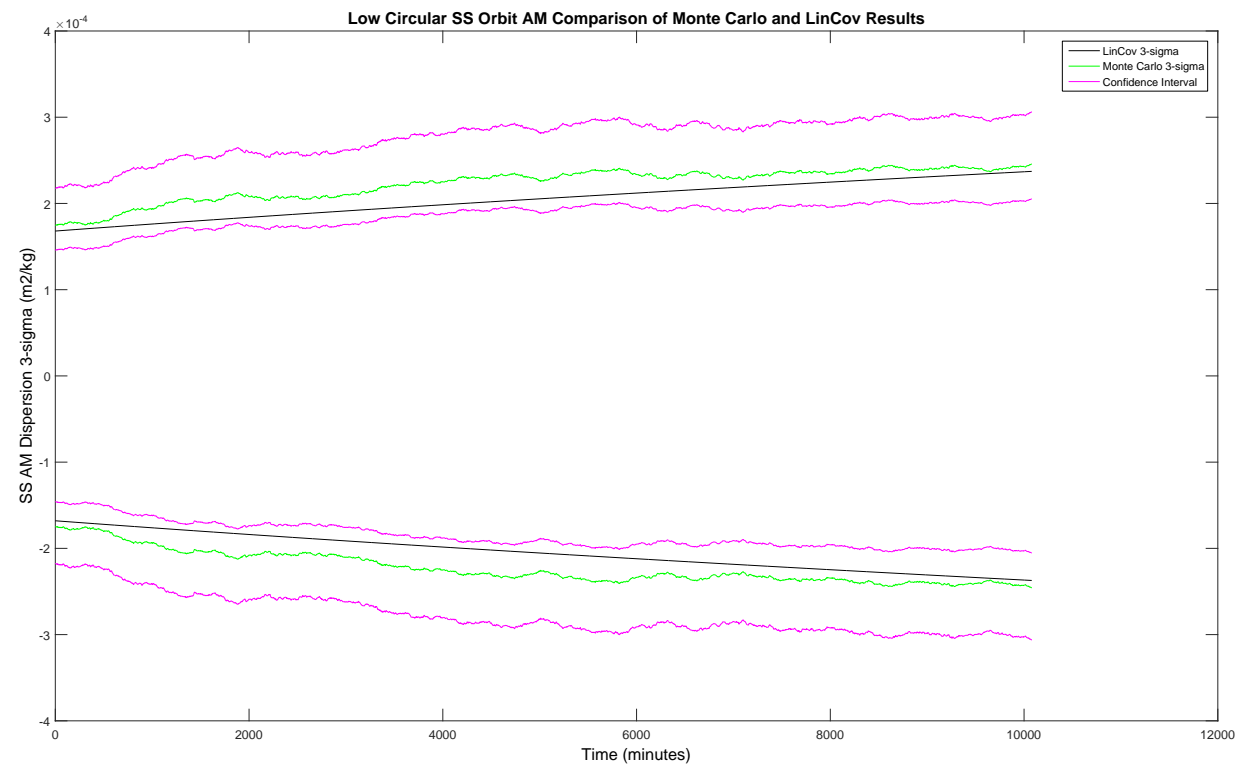

Fig. 9.19: Nominal Initial Errors - Ground Station and Low Circular SS Measurements, LinCov Validation, SS Area-to-Mass Ratio Estimation Error 

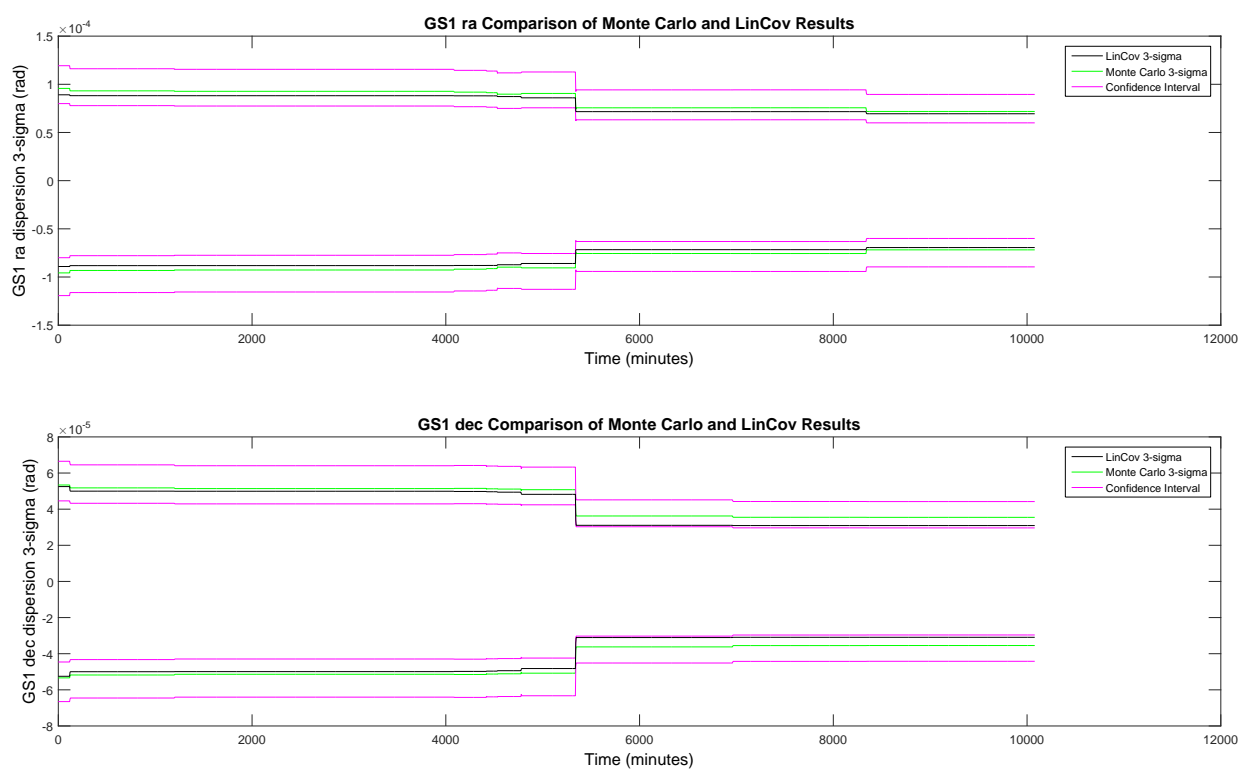

Fig. 9.20: Nominal Initial Errors - Ground Station and Low Circular SS Measurements, LinCov Validation, Ground Station 1 Biases Estimation Errors
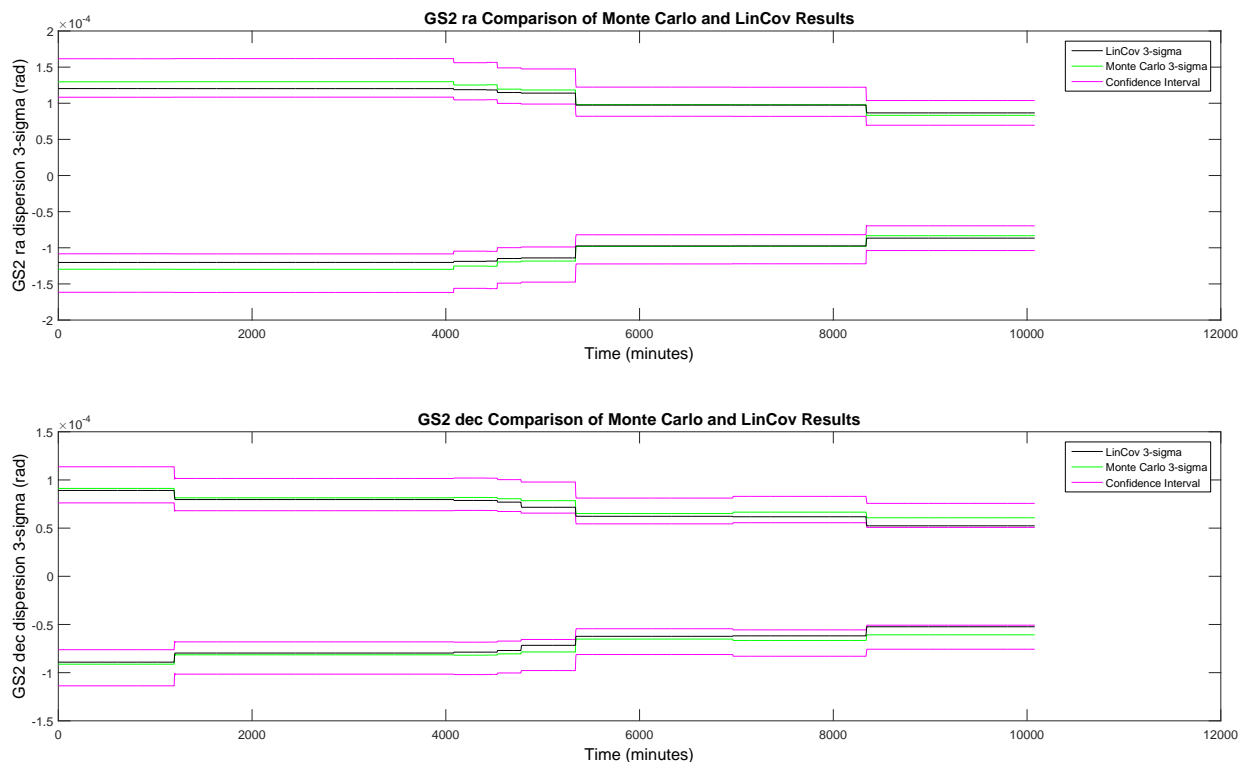

Fig. 9.21: Nominal Initial Errors - Ground Station and Low Circular SS Measurements, LinCov Validation, Ground Station 2 Biases Estimation Errors 

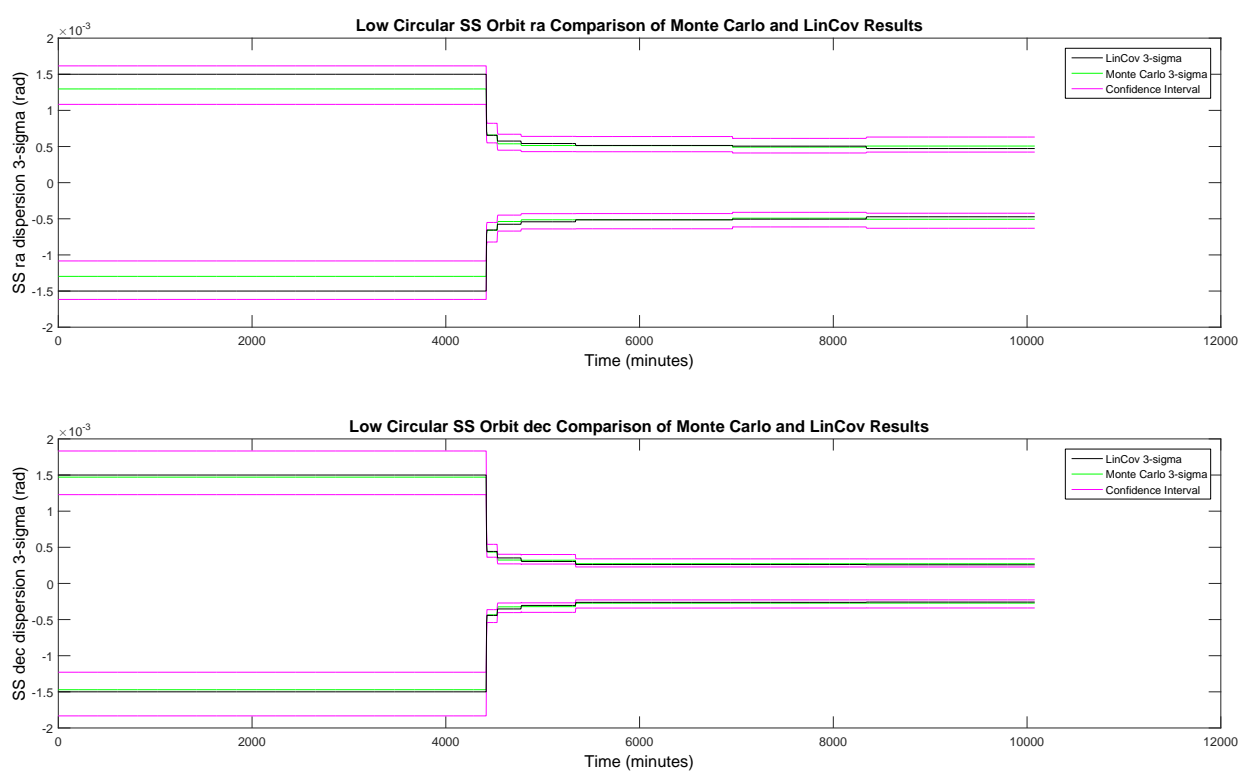

Fig. 9.22: Nominal Initial Errors - Ground Station and Low Circular SS Measurements, LinCov Validation, SS Biases Estimation Errors
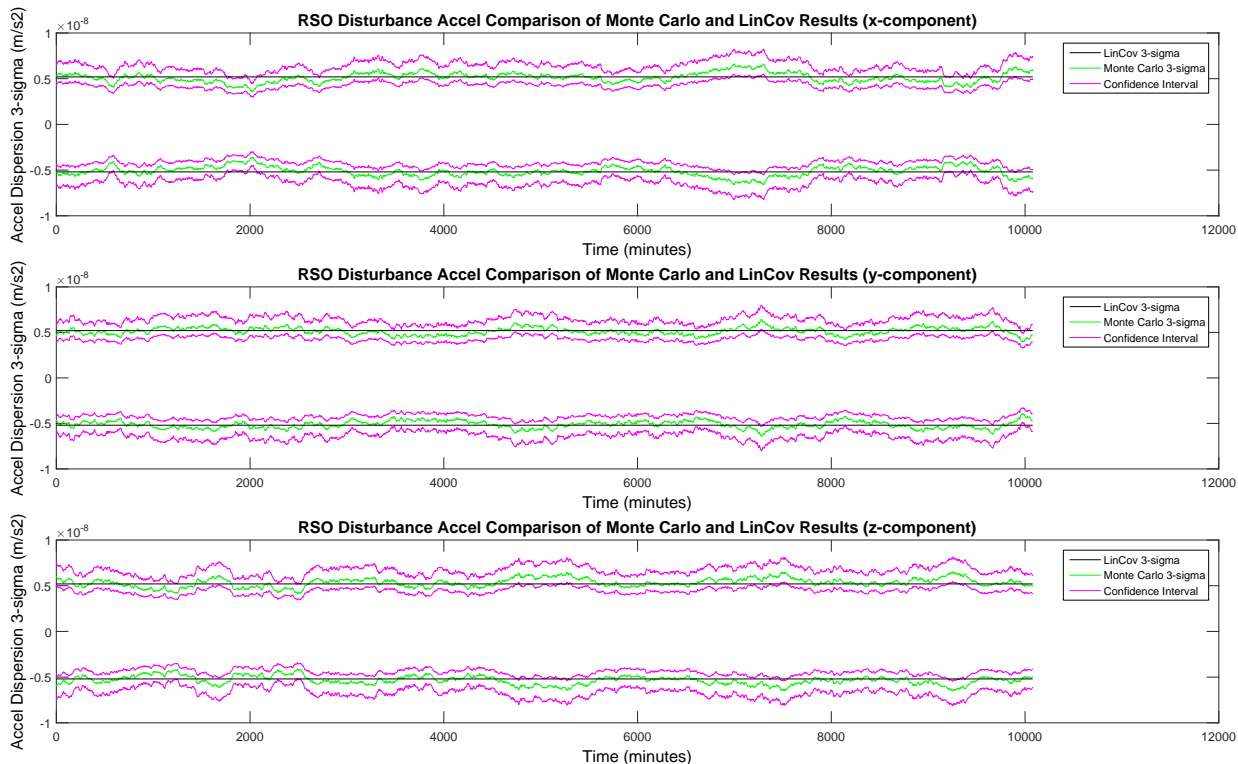

Fig. 9.23: Nominal Initial Errors - Ground Station and Low Circular SS Measurements, LinCov Validation, RSO Disturbance Accels Estimation Errors 

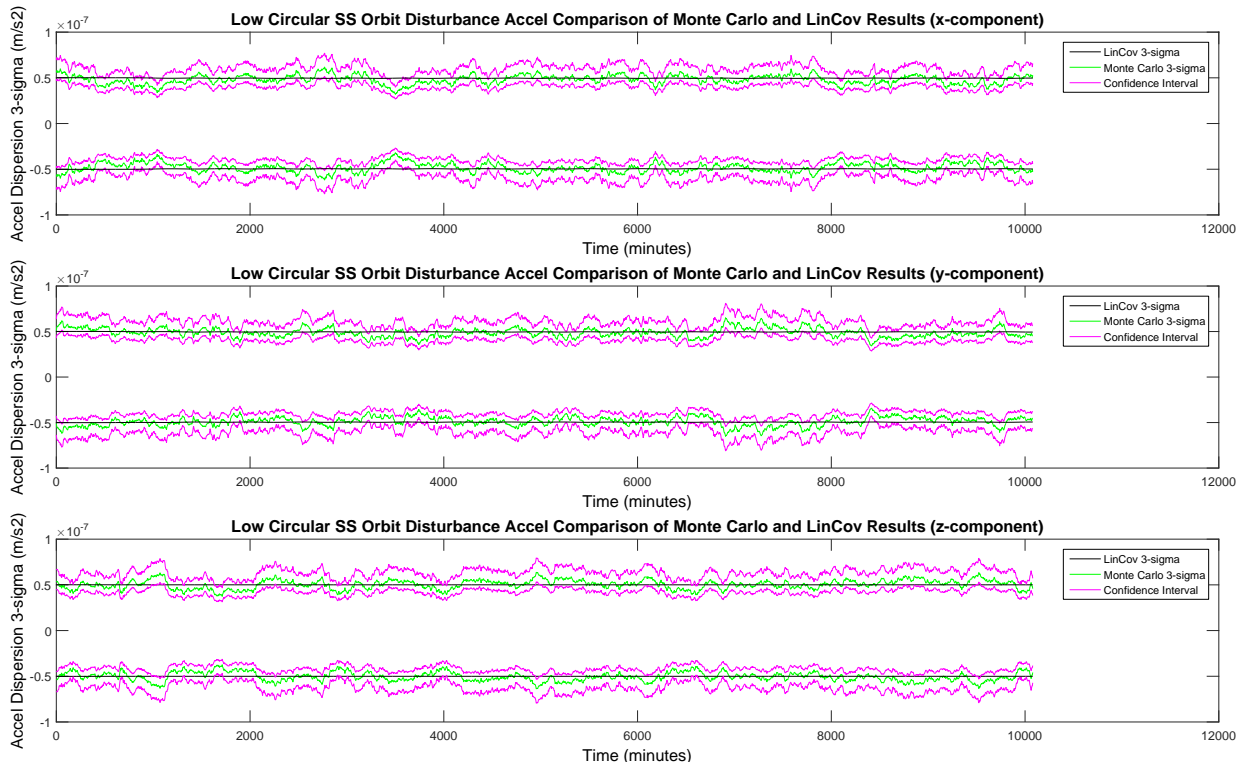

Fig. 9.24: Nominal Initial Errors - Ground Station and Low Circular SS Measurements, LinCov Validation, SS Disturbance Accels Estimation Errors

Figure 9.25 shows results from a low circular orbit space sensor and ground station measurements. All error sources are set to the high level. LinCov results stay within the Monte Carlo confidence intervals, other than the occasional outliers. 

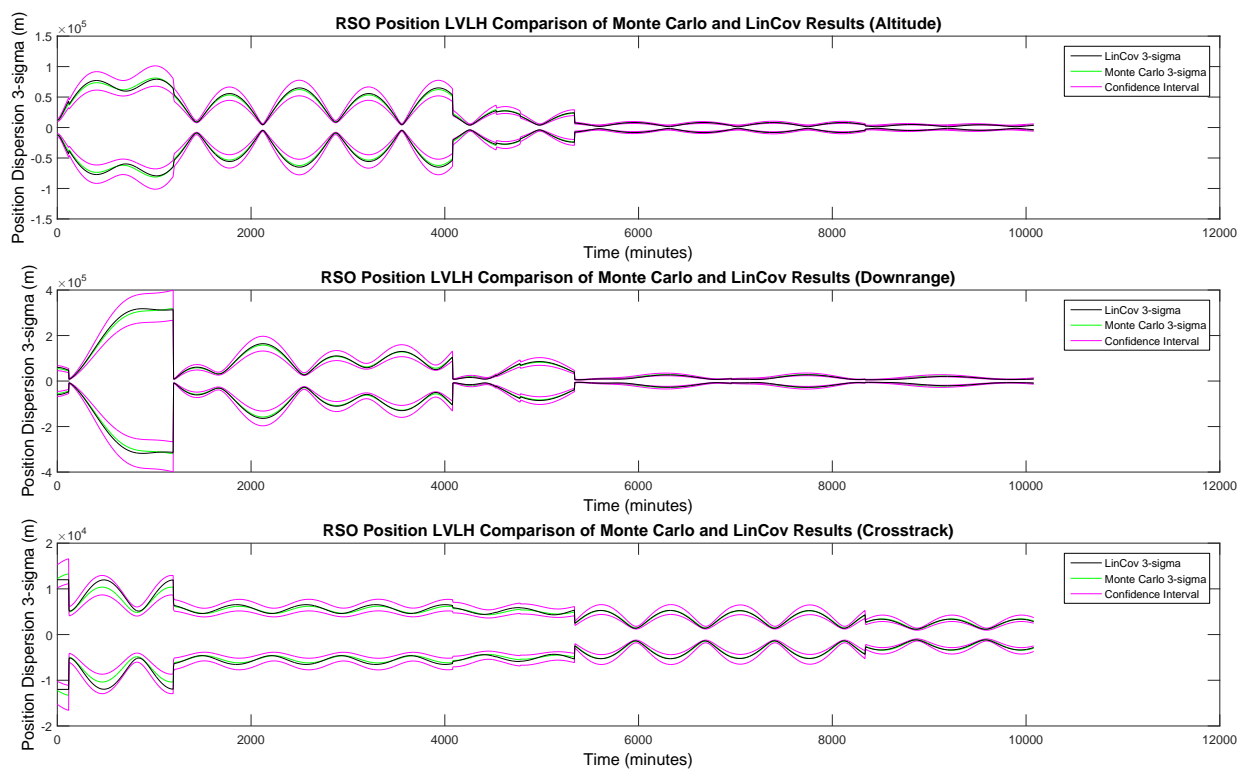

Fig. 9.25: High Initial Errors - Ground Station and Low Circular SS Measurements, LinCov Validation, RSO LVLH Position Estimation Errors

\subsubsection{High Circular Space Sensor Orbit}

Figure 9.26 shows results from a high circular orbit space sensor and ground station measurements. All error sources are set to the nominal level. LinCov results stay within the Monte Carlo confidence intervals, other than the occasional outliers. 

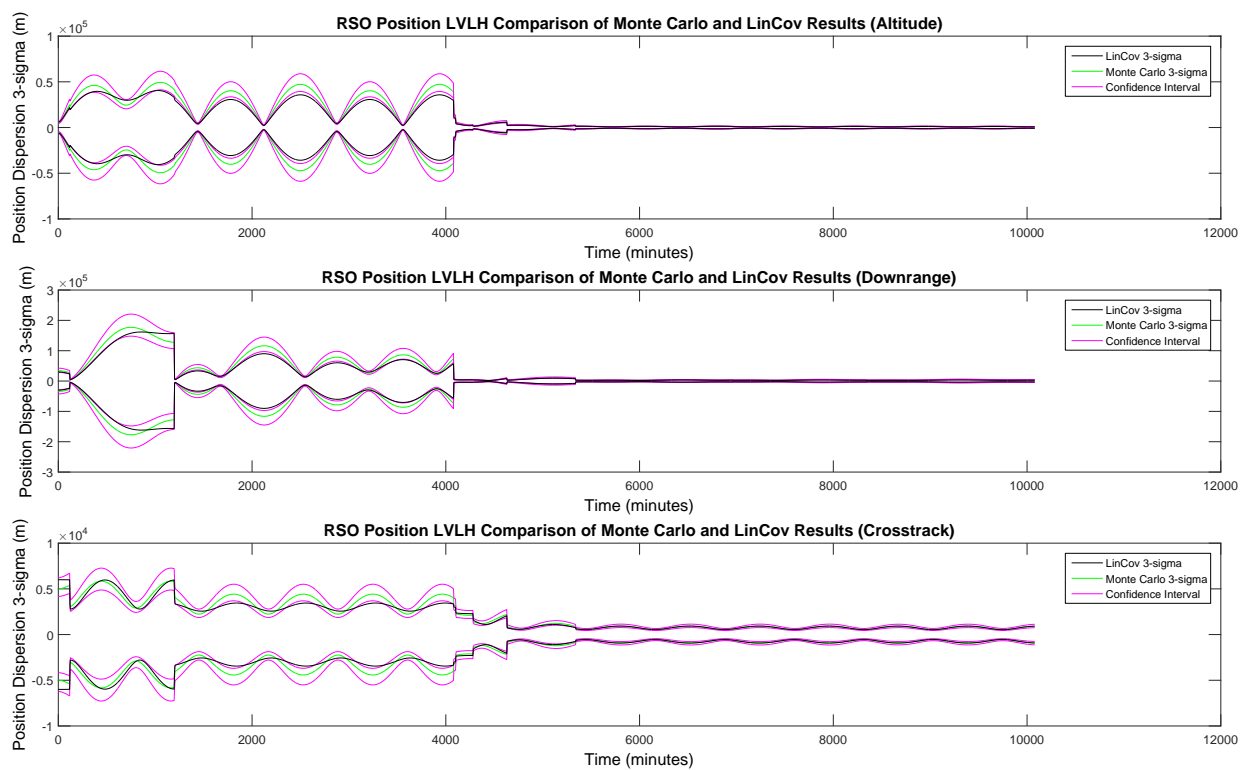

Fig. 9.26: Nominal Initial Errors - Ground Station and High Circular SS Measurements, LinCov Validation, RSO LVLH Position Estimation Errors

Figure 9.27 shows results from a high circular orbit space sensor and ground station measurements. All error sources are set to the high level. LinCov results stay within the Monte Carlo confidence intervals, other than the occasional outliers. 

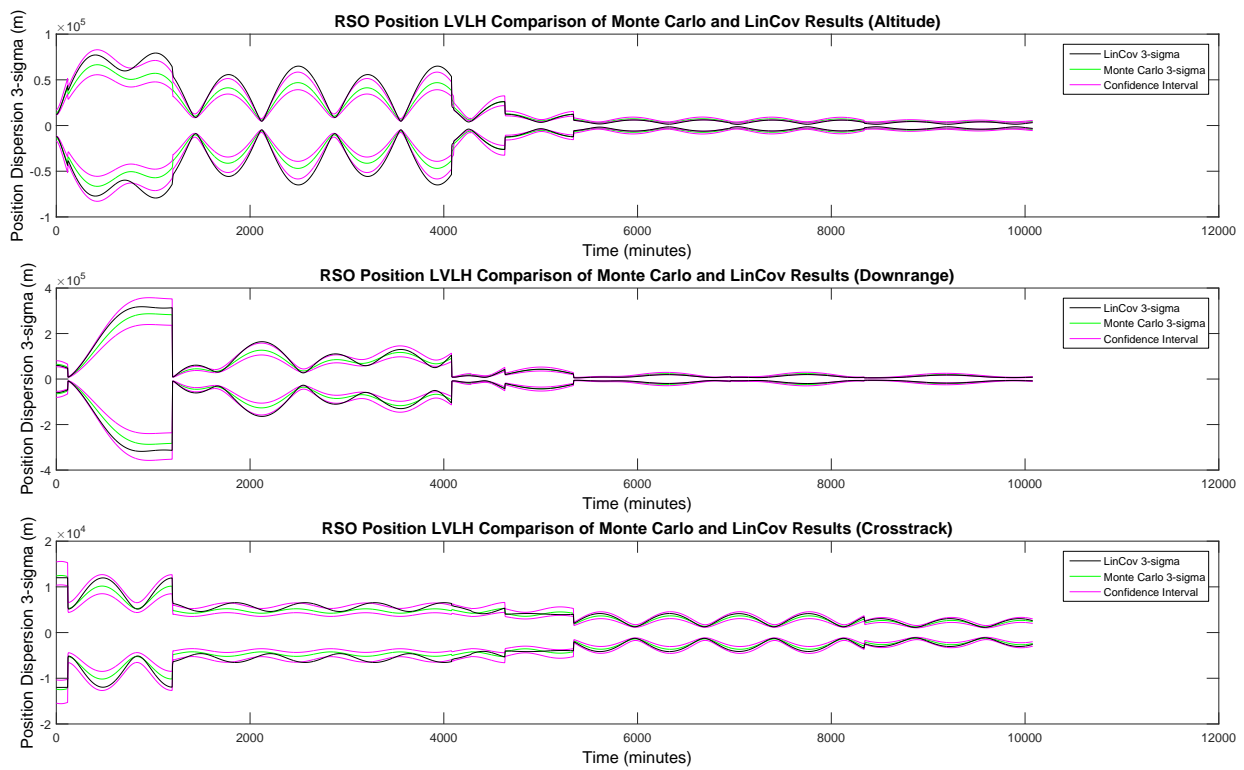

Fig. 9.27: High Initial Errors - Ground Station and High Circular SS Measurements, LinCov Validation, RSO LVLH Position Estimation Errors

\subsubsection{Elliptical Space Sensor Orbit}

Figure 9.28 shows results from an elliptical orbit space sensor and ground station measurements. All error sources are set to the nominal level. LinCov results stay within the Monte Carlo confidence intervals, other than the occasional outliers. 

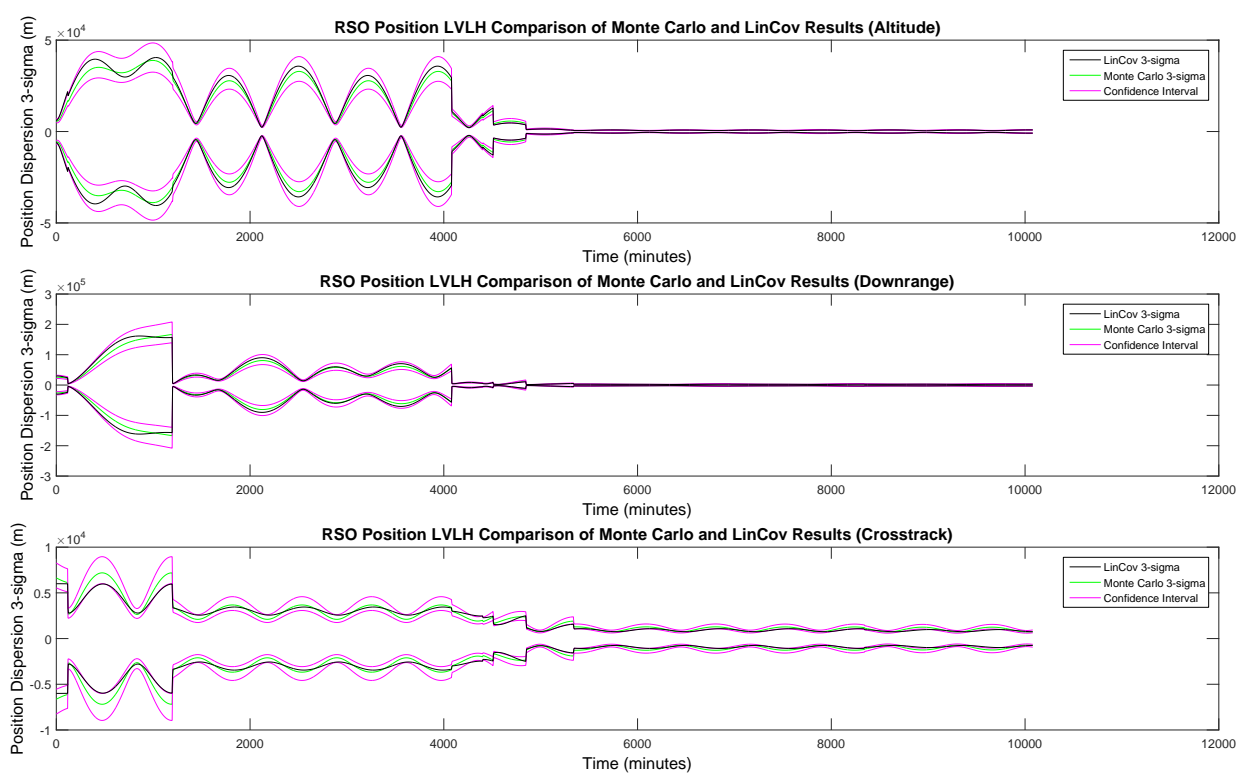

Fig. 9.28: Nominal Initial Errors - Ground Station and Elliptical SS Measurements, LinCov Validation, RSO LVLH Position Estimation Errors

Figure 9.29 shows results from an elliptical orbit space sensor and ground station measurements. All error sources are set to the high level. LinCov results stay within the Monte Carlo confidence intervals, other than the occasional outliers. 

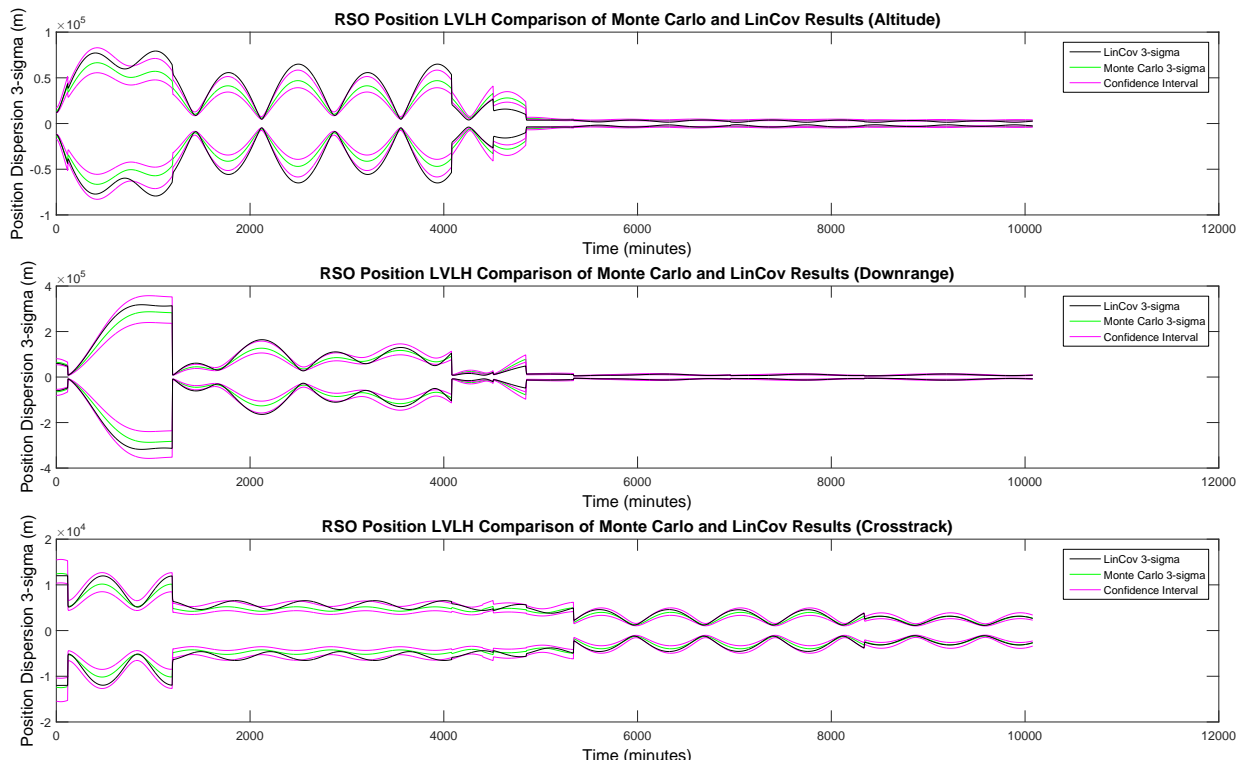

Fig. 9.29: High Initial Errors - Ground Station and Elliptical SS Measurements, LinCov Validation, RSO LVLH Position Estimation Errors

\subsection{Truth Environment Verification}

The following validation runs incorporate space sensor and ground station measurements of the RSO. Ground station measurements alone, and each space sensor orbit-low circular, high circular, and elliptical-are analyzed for low error source levels. The space sensor position and velocity is updated through the use of GPS. The truth model incorporates an $18 \times 18$ gravity model without disturbance accelerations and LinCov incorporates a $2 \times 2$ gravity model with disturbance accelerations. All relevant plots are shown for the ground station measurement only and low circular space sensor orbit with low error sources. The remaining analysis results show plots for the research critical results, the RSO position error in LVLH, as well as the space sensor disturbance acceleration. 


\subsubsection{Ground Station Measurements - Low Errors}

Figures 9.30 through 9.34 are results from LinCov analysis of the RSO with ground station measurements. All error sources are set to the low level. The main difference between these results and the results from the truth model $2 \times 2$ gravity analysis is seen in the disturbance acceleration plot of the RSO. It can be seen that the LinCov disturbance acceleration lies outside of the Monte Carlo confidence intervals. The LinCov disturbance acceleration model was built to be conservative, just as it was in the EKF. It slightly overestimates the effect of the disturbance accelerations in all scenarios. The Monte Carlo/LinCov results for the Ground Station 2 bias were nearly identical to Ground Station 1 results.
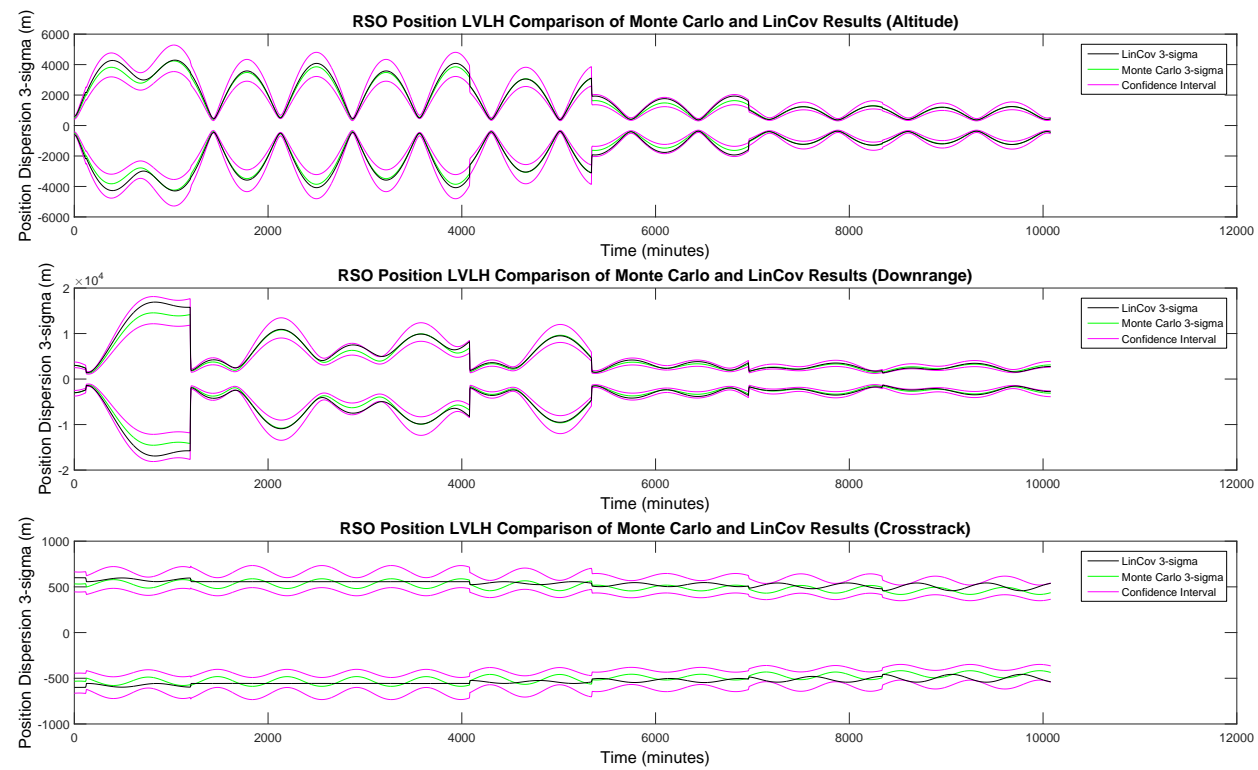

Fig. 9.30: Low Initial Errors - Ground Station Only Measurements, LinCov Validation, RSO LVLH Position Estimation Errors 

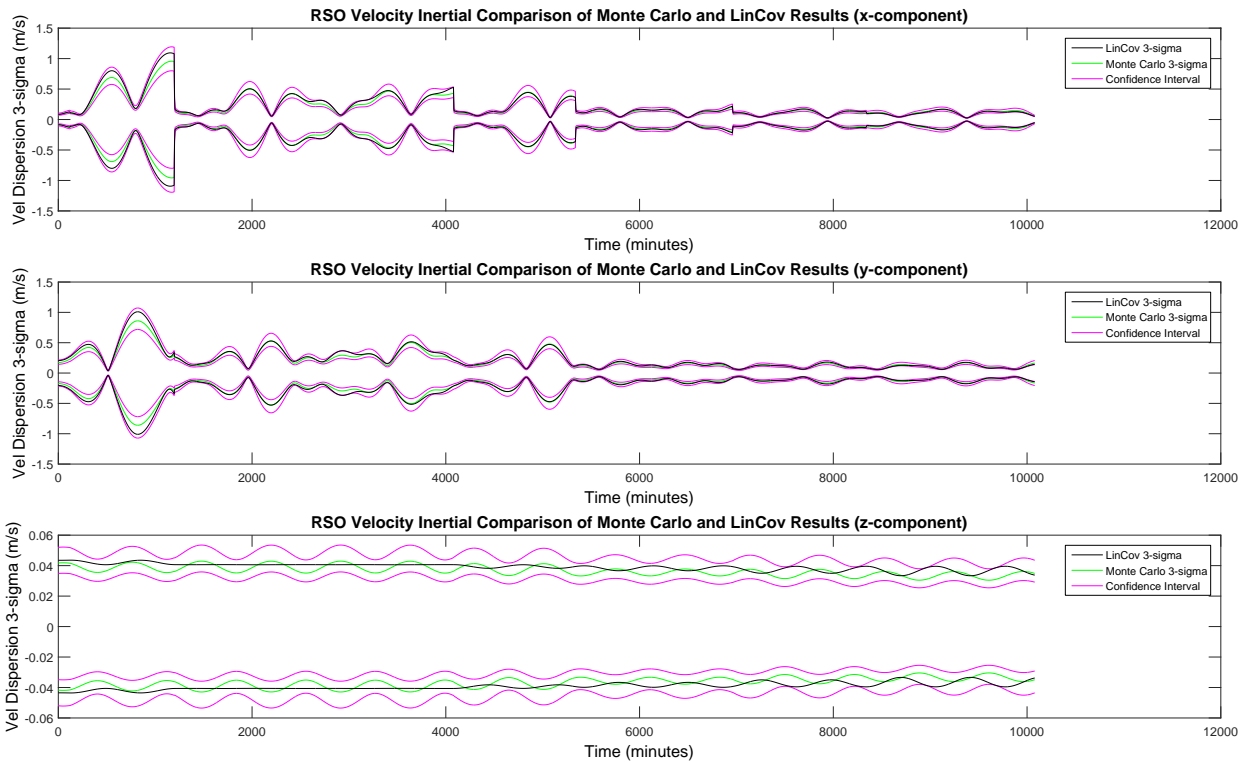

Fig. 9.31: Low Initial Errors - Ground Station Only Measurements, LinCov Validation, RSO Inertial Velocity Estimation Errors

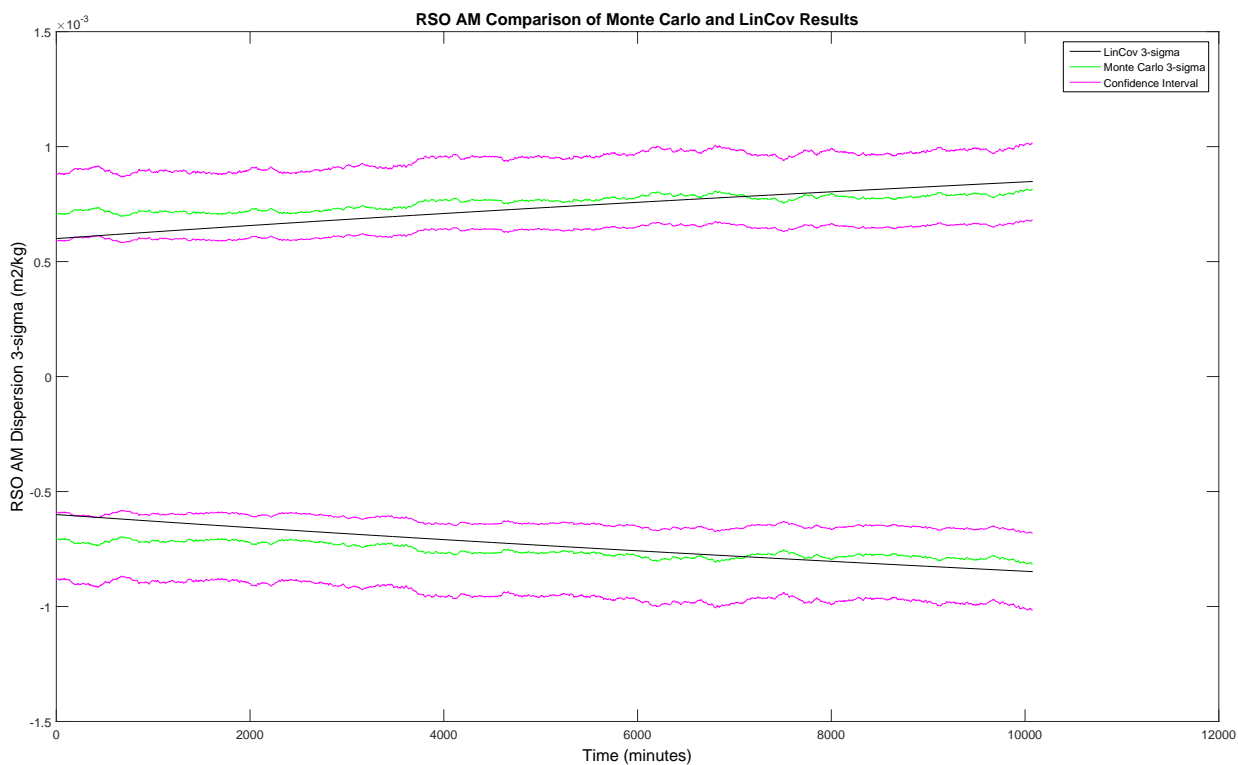

Fig. 9.32: Low Initial Errors - Ground Station Only Measurements, LinCov Validation, RSO Area-to-Mass Ratio Estimation Error 

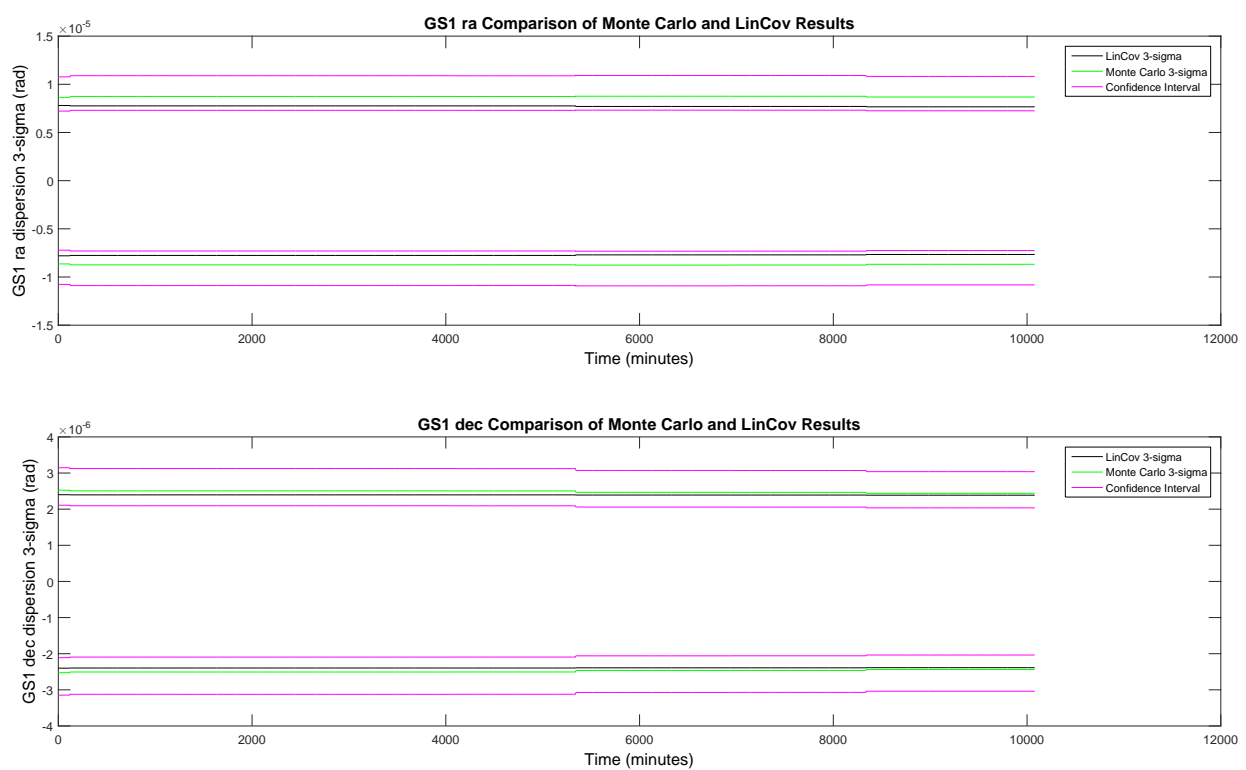

Fig. 9.33: Low Initial Errors - Ground Station Only Measurements, LinCov Validation, Ground Station 1 Biases Estimation Errors
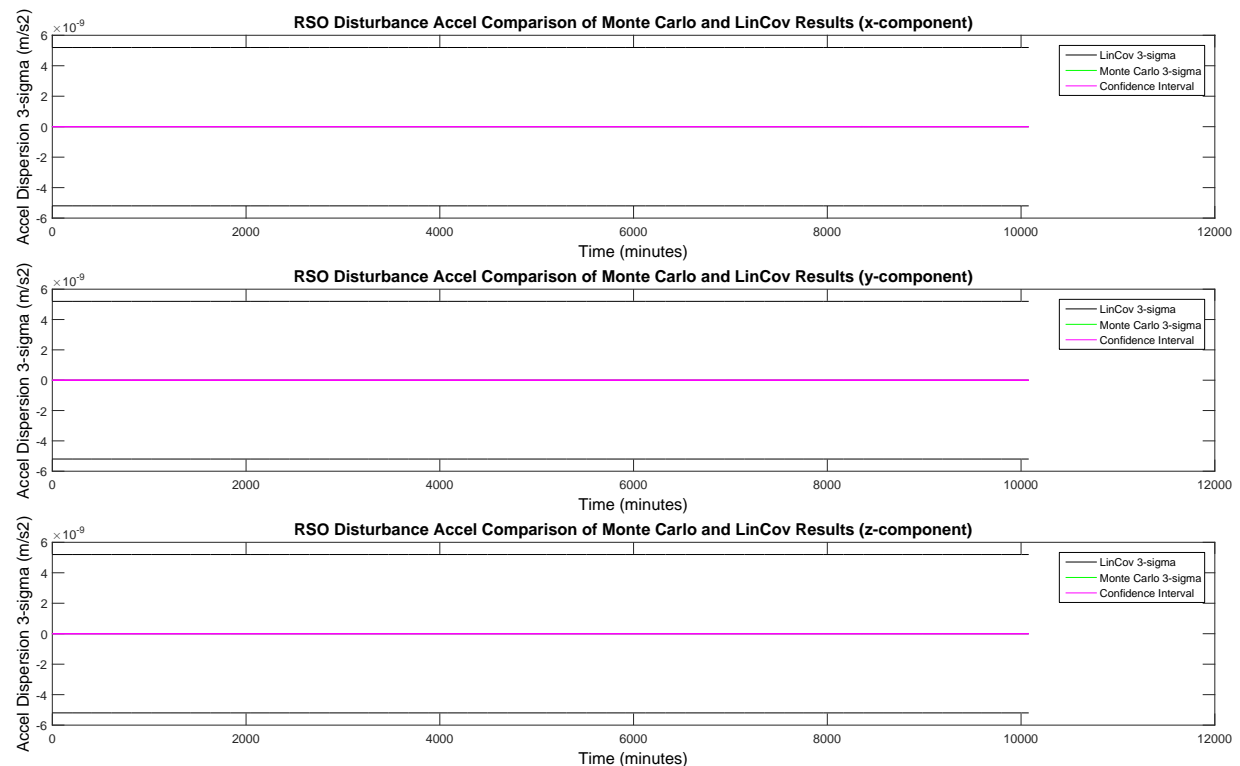

Fig. 9.34: Low Initial Errors - Ground Station Only Measurements, LinCov Validation, RSO Disturbance Accels Estimation Errors 


\subsubsection{Low Circular Space Sensor Orbit}

Figures 9.35 through 9.45 are results from LinCov analysis of the RSO with low circular orbit space sensor and ground station measurements. All error sources are set to the low level. The main differences between these results and the results from the truth model $2 \times 2$ gravity analysis are seen in the disturbance acceleration plots of the RSO and space sensor. It can be seen that the LinCov disturbance acceleration lies outside of the Monte Carlo confidence intervals. The LinCov disturbance acceleration model was built to be conservative, just as it was in the EKF. It slightly overestimates the effect of the disturbance accelerations in all scenarios.
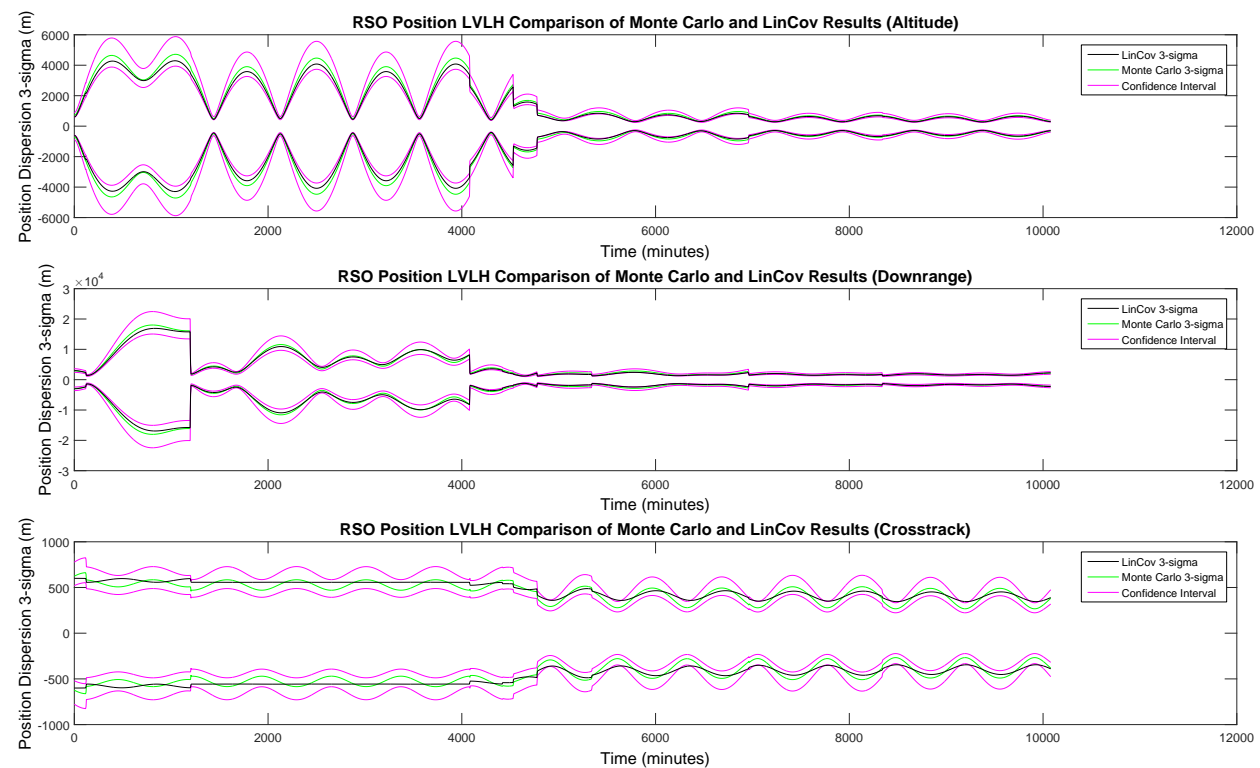

Fig. 9.35: Low Initial Errors - Ground Station and Low Circular SS Measurements, LinCov Validation, RSO LVLH Position Estimation Errors 

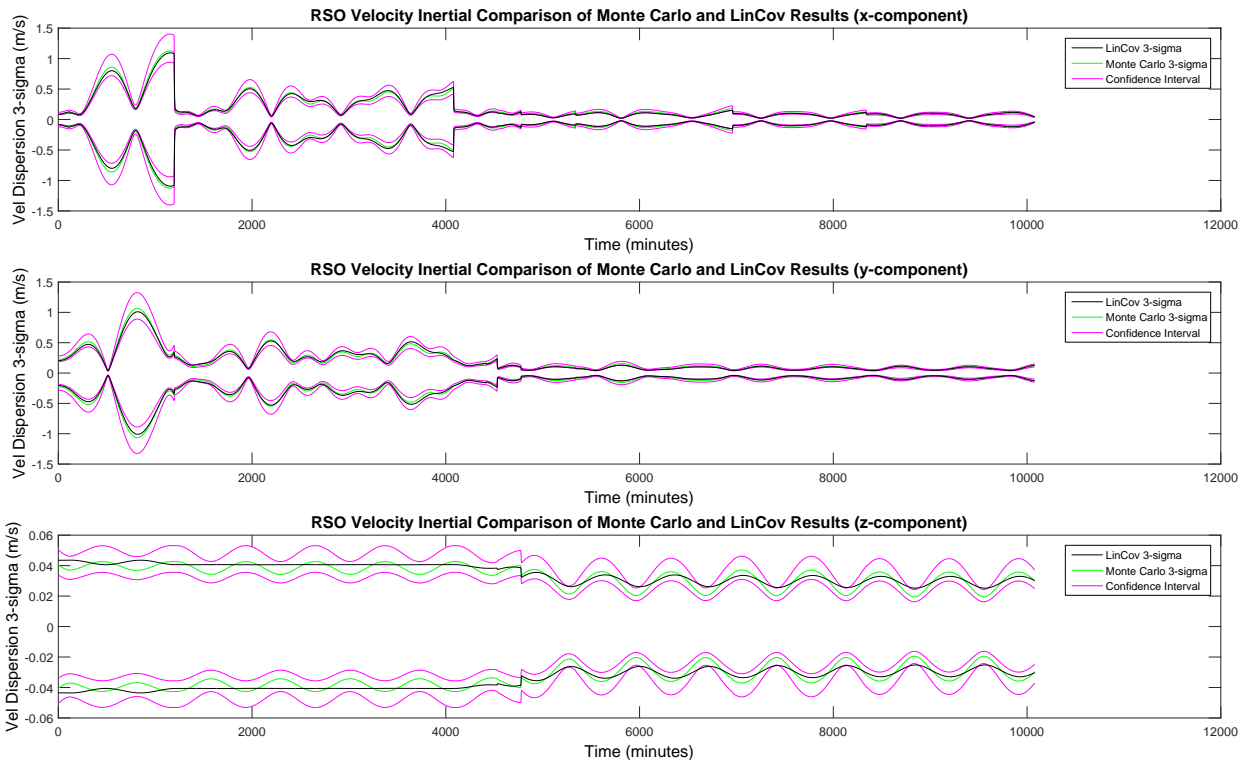

Fig. 9.36: Low Initial Errors - Ground Station and Low Circular SS Measurements, LinCov Validation, RSO Inertial Velocity Estimation Errors

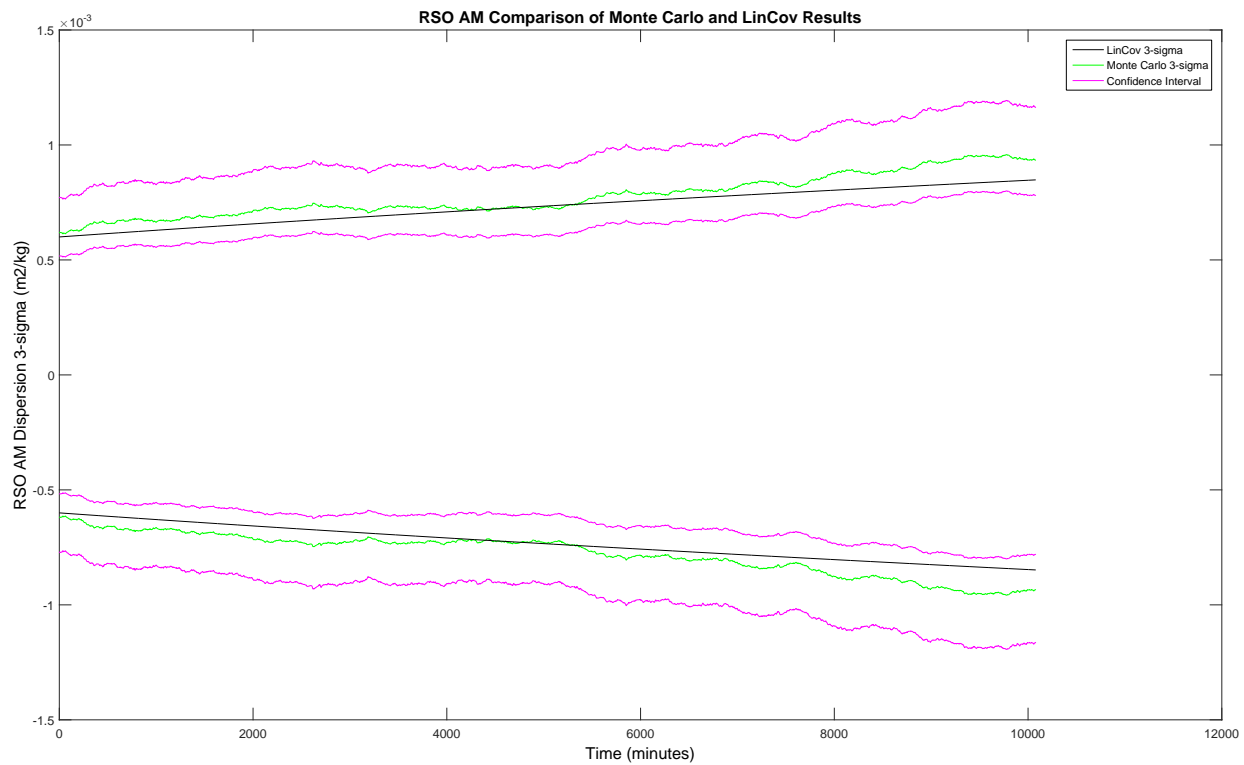

Fig. 9.37: Low Initial Errors - Ground Station and Low Circular SS Measurements, LinCov Validation, RSO Area-to-Mass Ratio Estimation Error 

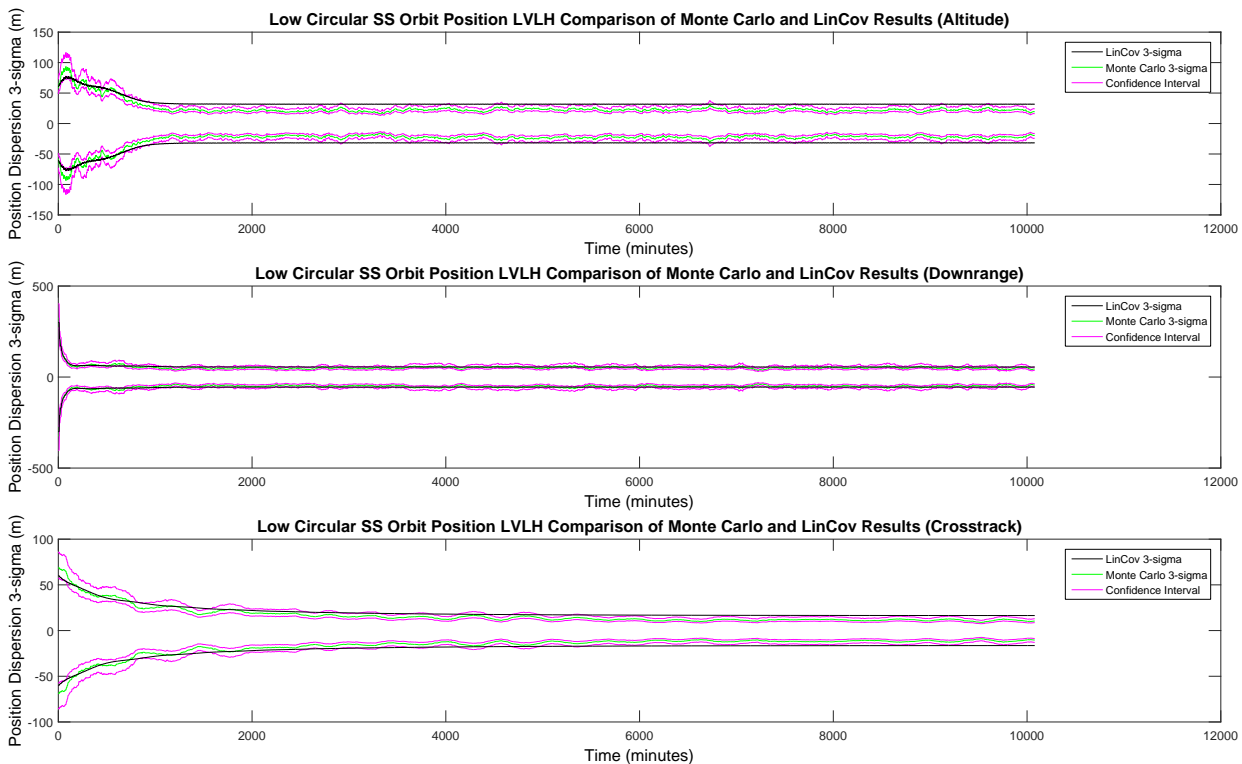

Fig. 9.38: Low Initial Errors - Ground Station and Low Circular SS Measurements, LinCov Validation, SS LVLH Position Estimation Errors
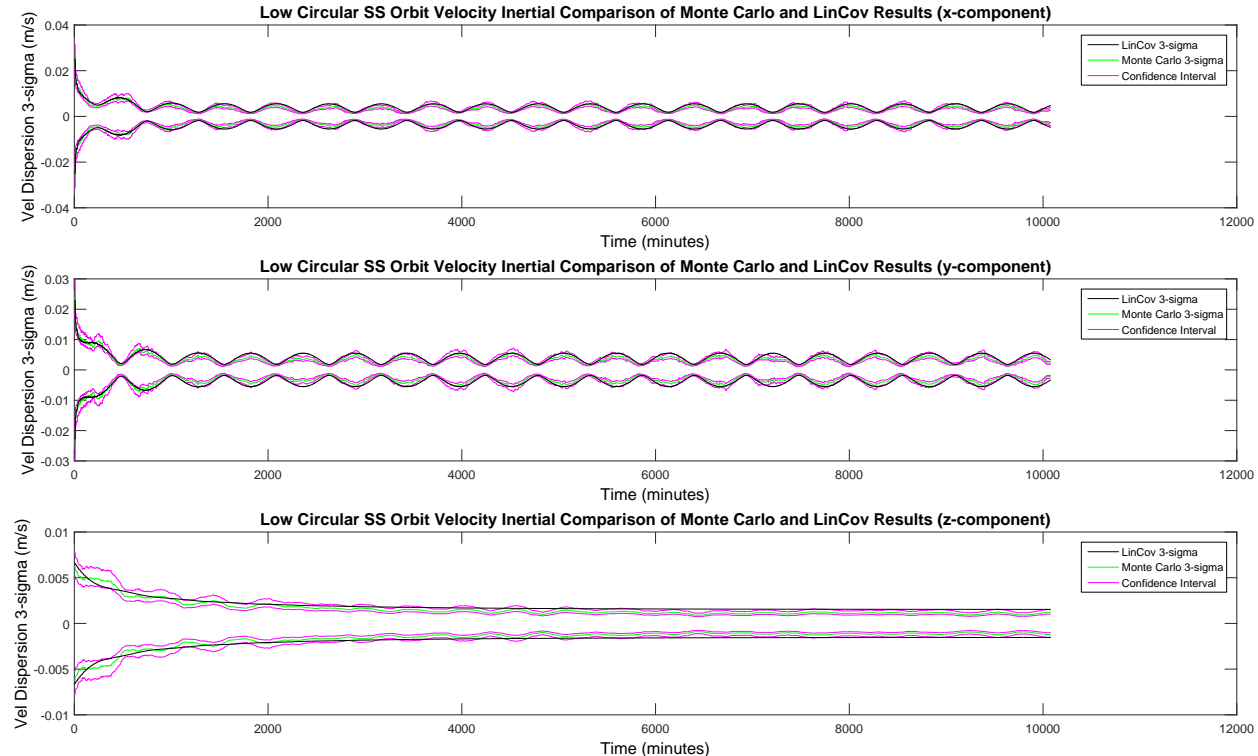

Fig. 9.39: Low Initial Errors - Ground Station and Low Circular SS Measurements, LinCov Validation, SS Inertial Velocity Estimation Errors 


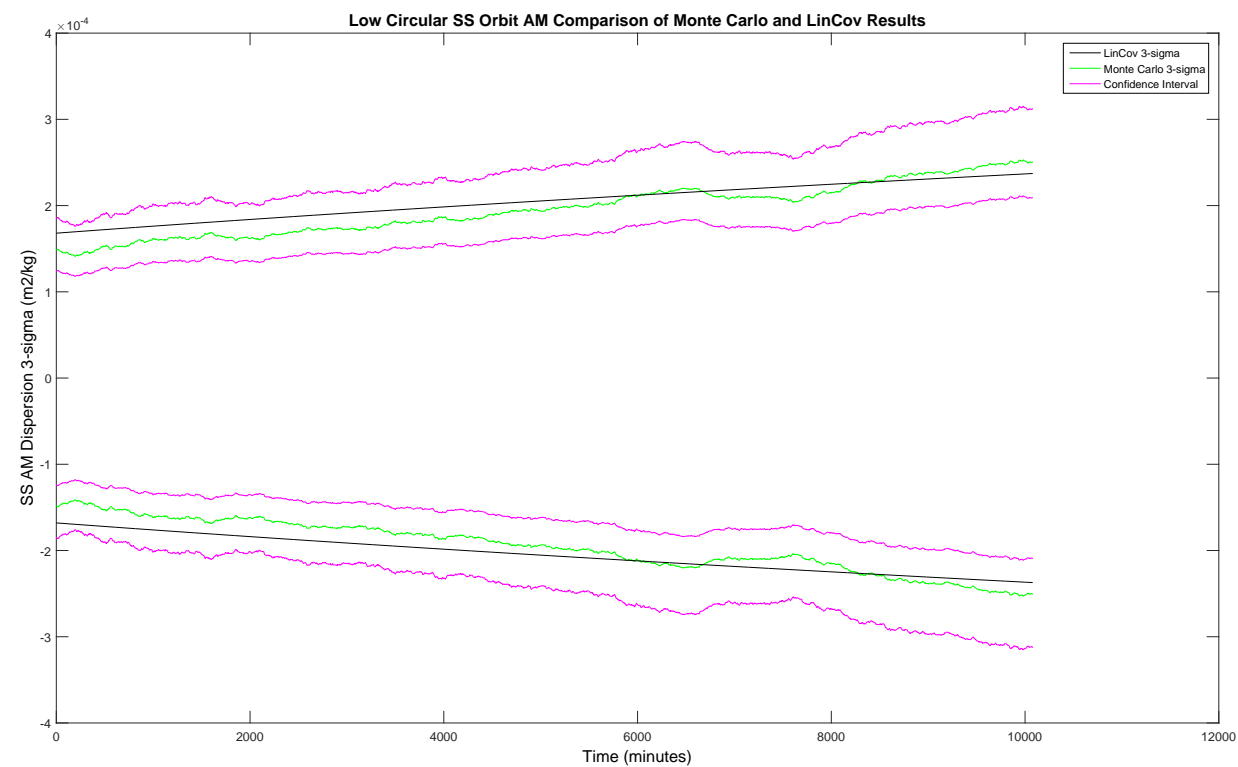

Fig. 9.40: Low Initial Errors - Ground Station and Low Circular SS Measurements, LinCov Validation, SS Area-to-Mass Ratio Estimation Error
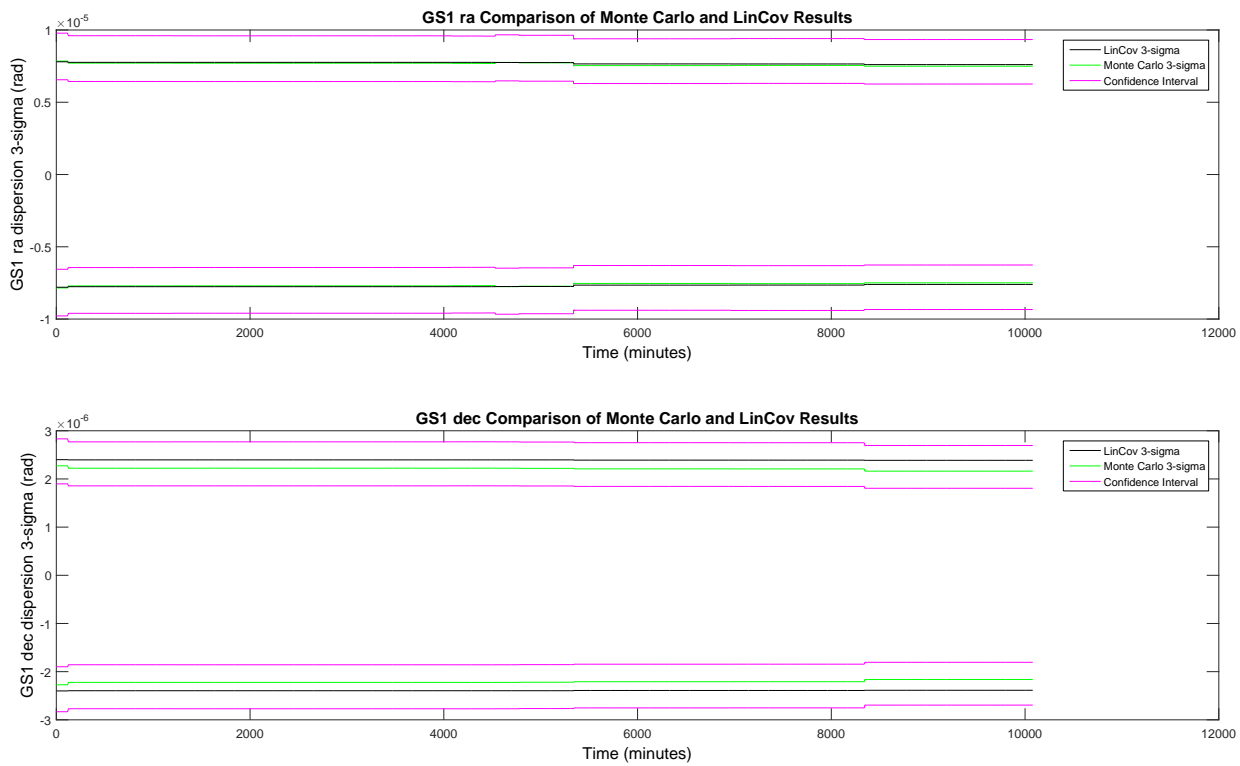

Fig. 9.41: Low Initial Errors - Ground Station and Low Circular SS Measurements, LinCov Validation, Ground Station 1 Biases Estimation Errors 

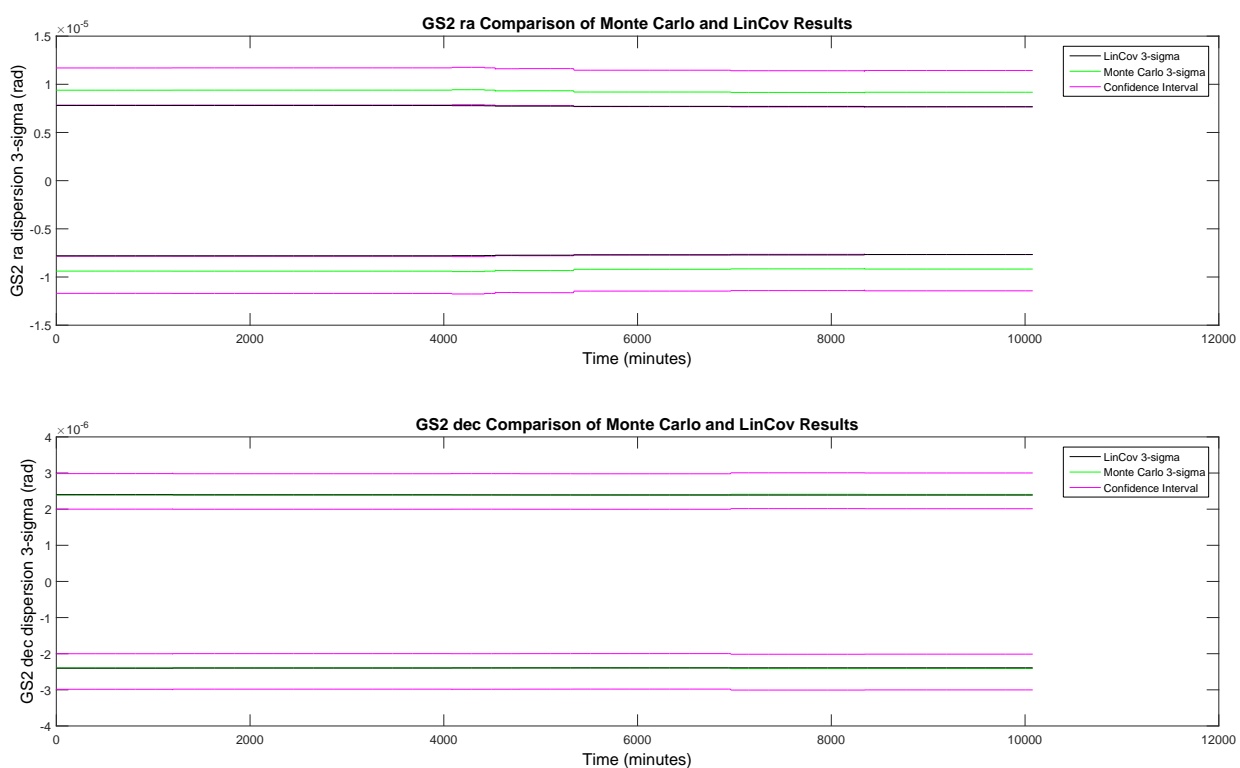

Fig. 9.42: Low Initial Errors - Ground Station and Low Circular SS Measurements, LinCov Validation, Ground Station 2 Biases Estimation Errors
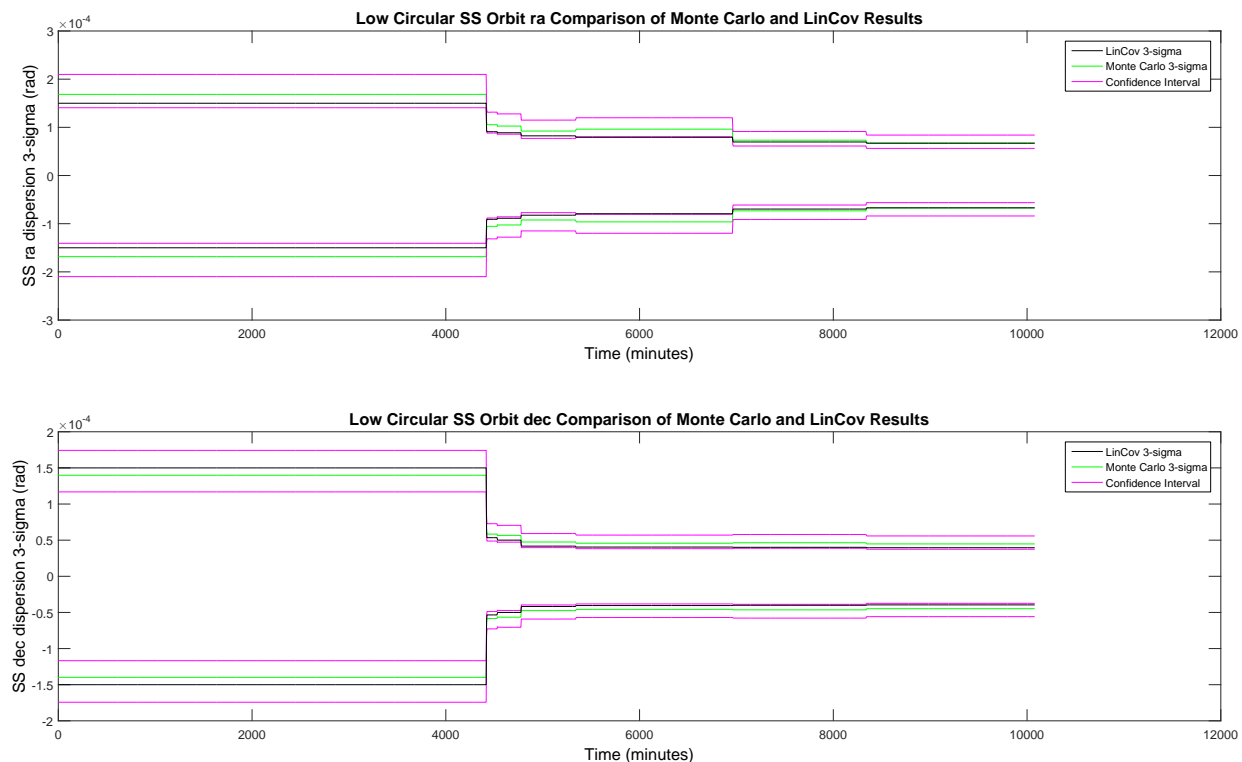

Fig. 9.43: Low Initial Errors - Ground Station and Low Circular SS Measurements, LinCov Validation, SS Biases Estimation Errors 

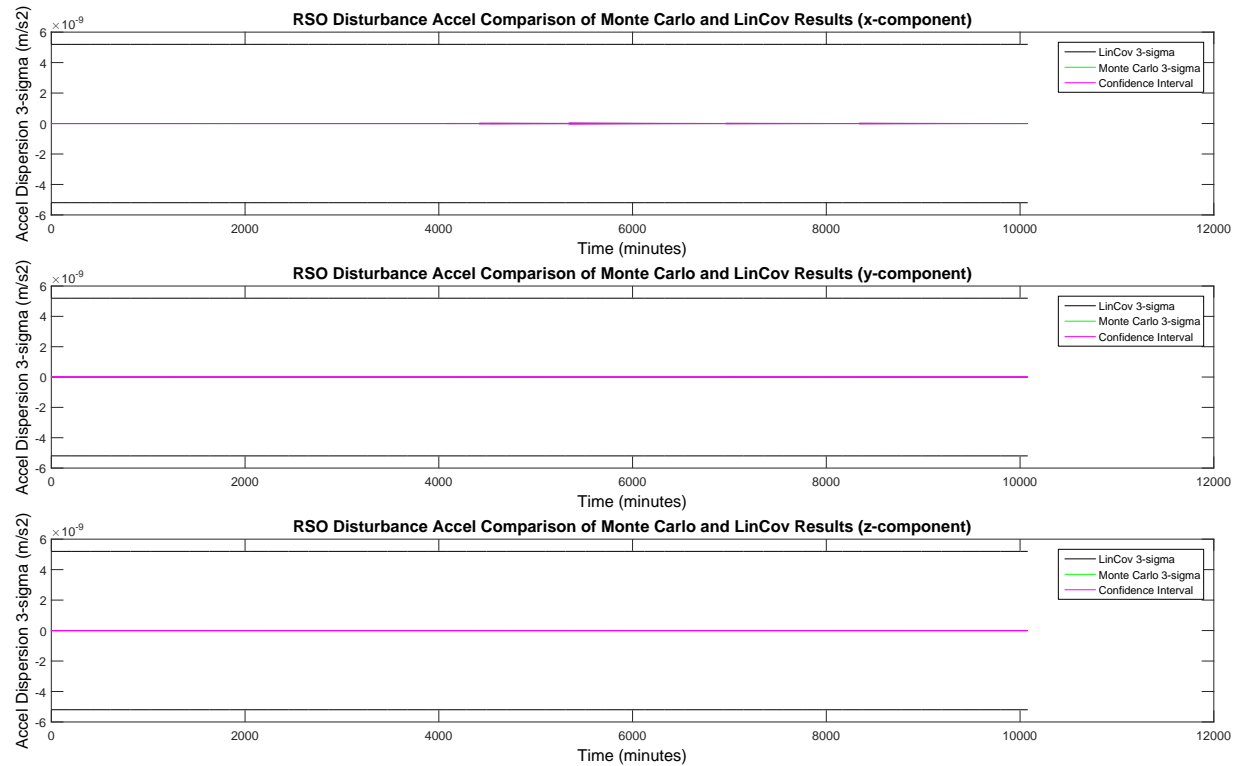

Fig. 9.44: Low Initial Errors - Ground Station and Low Circular SS Measurements, LinCov Validation, RSO Disturbance Accels Estimation Errors
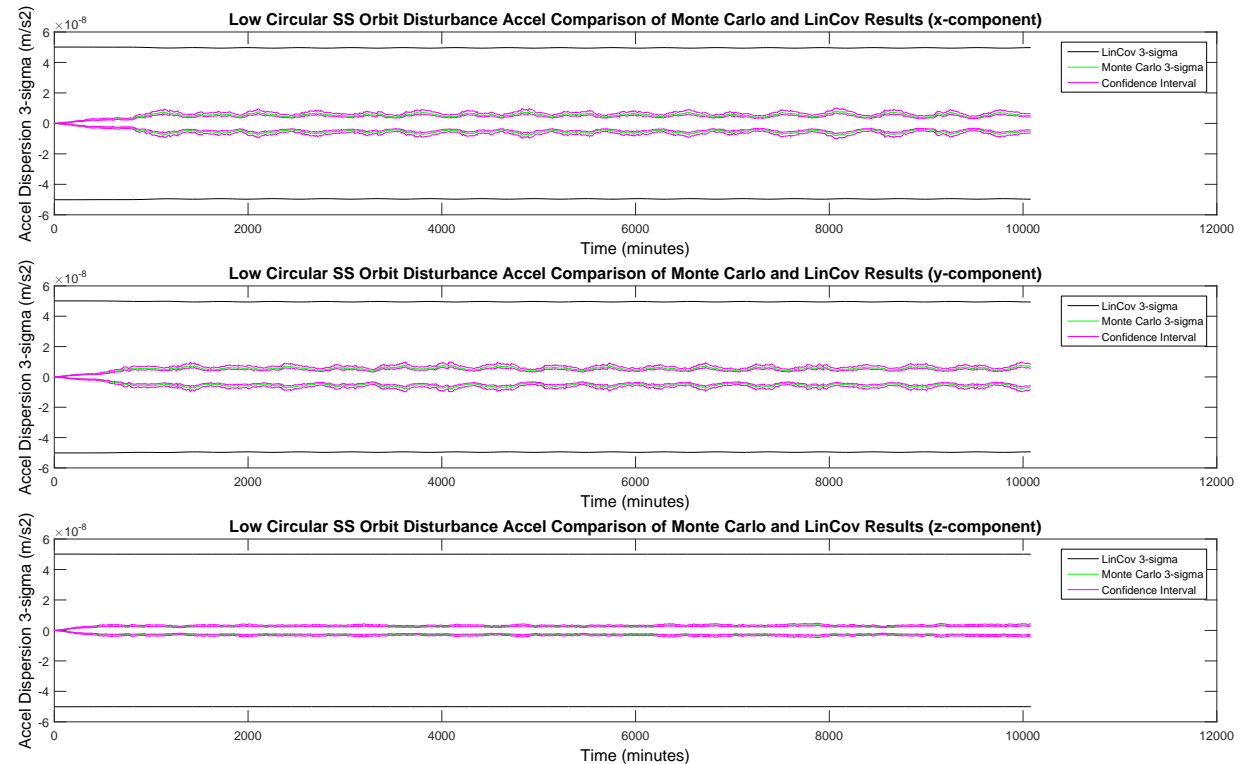

Fig. 9.45: Low Initial Errors - Ground Station and Low Circular SS Measurements, LinCov Validation, SS Disturbance Accels Estimation Errors 


\subsubsection{High Circular Space Sensor Orbit}

Figures 9.46 and 9.47 are results from LinCov analysis of the RSO with high circular orbit space sensor and ground station measurements. All error sources are set to the low level. The LinCov disturbance acceleration lies outside of the Monte Carlo confidence intervals.
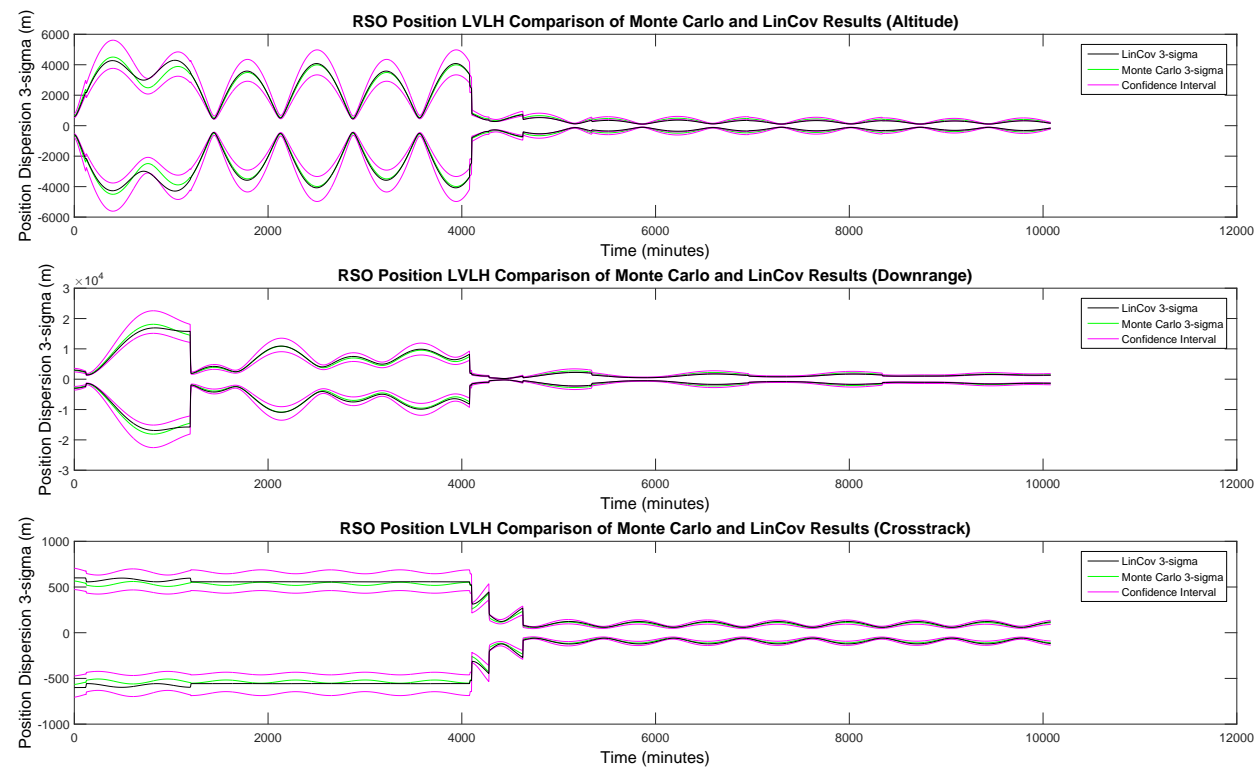

Fig. 9.46: Low Initial Errors - Ground Station and High Circular SS Measurements, LinCov Validation, RSO LVLH Position Measurement Errors 

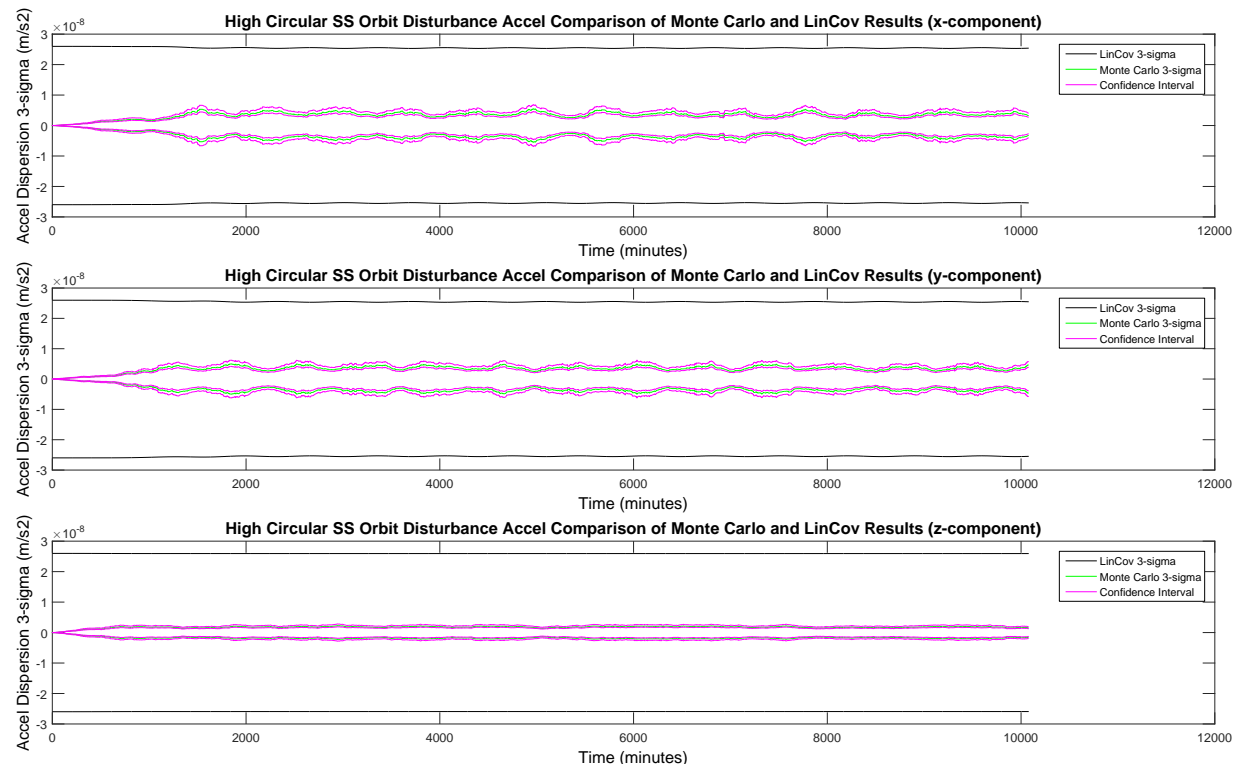

Fig. 9.47: Low Initial Errors - Ground Station and High Circular SS Measurements, LinCov Validation, SS Disturbance Accels Estimation Errors

\subsubsection{Elliptical Space Sensor Orbit}

Figures 9.48 and 9.49 are results from LinCov analysis of the RSO with elliptical orbit space sensor and ground station measurements. All error sources are set to the low level. The LinCov disturbance acceleration lies outside of the Monte Carlo confidence intervals. 

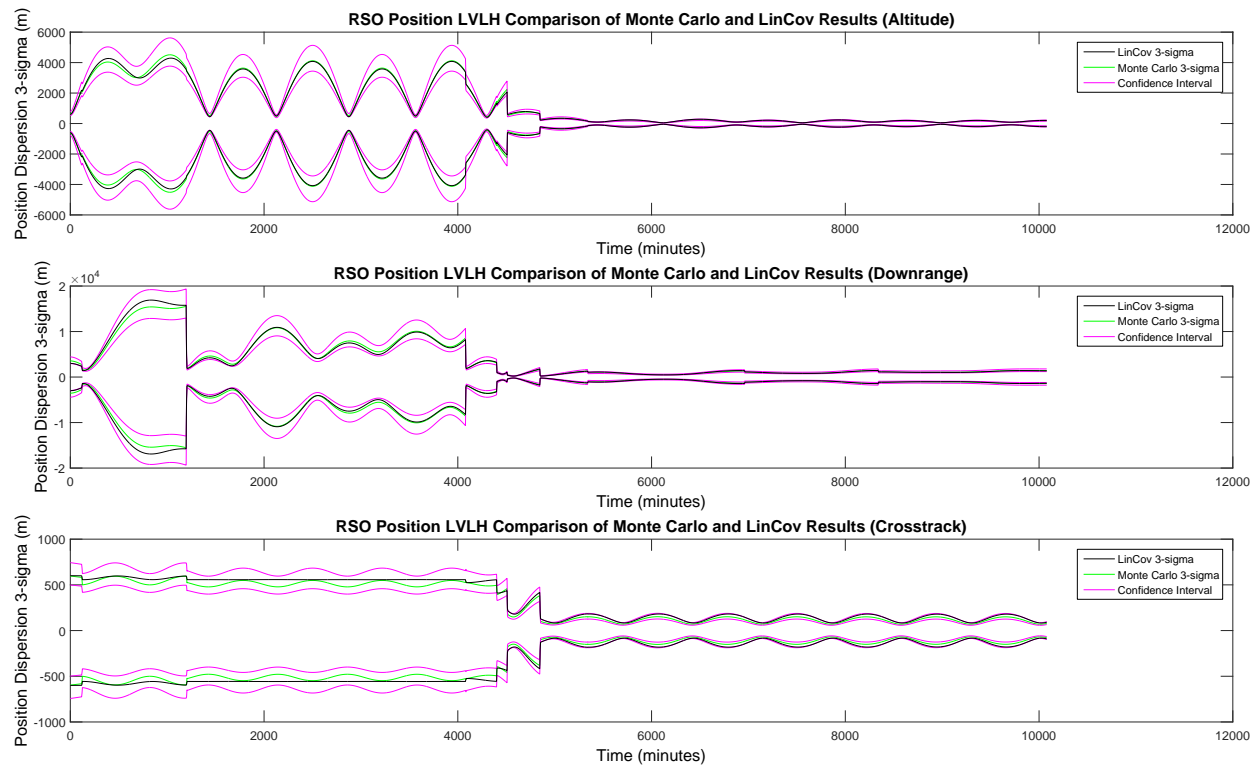

Fig. 9.48: Low Initial Errors - Ground Station and Elliptical SS Measurements, LinCov Validation, RSO LVLH Position Measurement Errors
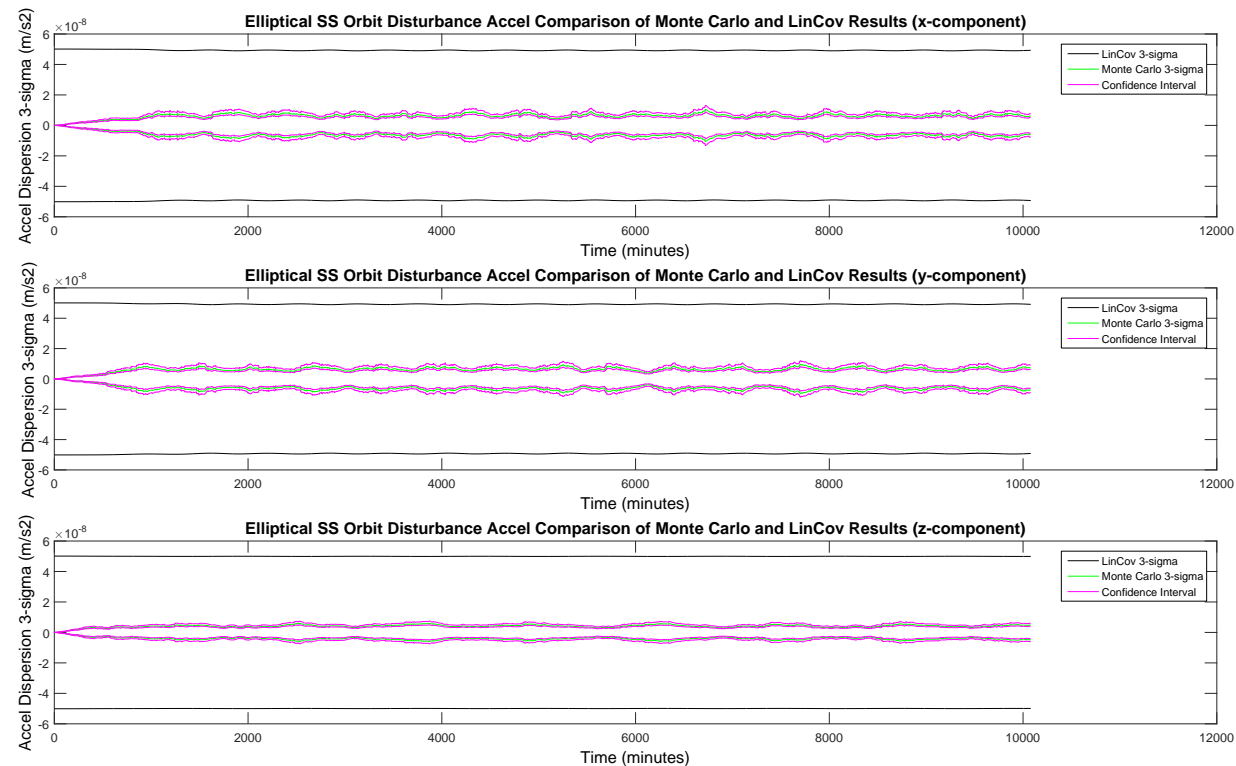

Fig. 9.49: Low Initial Errors - Ground Station and Elliptical SS Measurements, LinCov Validation, SS Disturbance Accels Estimation Errors 


\subsection{Summary}

This chapter validated the LinCov analysis tool developed in Chapter 8. To validate the LinCov analysis tool, the same set of verification runs used to validate the EKF were applied to the LinCov tool. Now, the LinCov analysis tool can be used to generate RSO estimation results. 
CHAPTER 10

\section{RESULTS - SPACE SENSOR AUGMENTATION BASELINE RESULTS}

\subsection{Results Overview}

The goal of the following results chapters is to quantify the increased orbital accuracy of objects in the GEO catalog that is achieved when the AFSPC SSN is augmented with space-based angles-only measurements from a sensor in a near-GEO orbit. The AFSPC SSN ground stations utilized are Socorro, New Mexico and Maui, Hawaii. All three space sensor orbits: low circular, high circular, and elliptical, defined in Chapter 3, will be utilized in the analysis. The analysis is broken into 7 different categories, each investigating a different aspect of the research problem. The seven categories are:

1) Baseline ground station and space sensor results utilizing the nominal tasking profiles with low, nominal, and high error source levels

2) Varying the number of ground station measurement periods

3) Extending the duration of the space sensor measurement periods

4) Studying the effects of an inclined RSO versus not inclined

5) Studying the effects of an inclined space sensor orbit

6) Varying the accuracy of the GPS space sensor updates

7) Using the USN to update the space sensor position and velocity

This chapter will study the baseline analysis results. First, the ground station only results will be quantified. Then, the results achievable with space sensor augmentation are presented. RSO position results are presented after 1 week and 2 weeks of simulation. Ground station and space sensor measurement periods all occur within the first week of simulation, as presented in Chapter 3. GPS updates of the space sensor position and velocity continue through the second week of the simulation period. The results of this chapter are focused purely on the position accuracy achieved for the RSO. 


\subsection{Ground Station Measurements Only}

Presented in Figures 10.1 through 10.3 is the achievable RSO position accuracy at 1 week and 2 weeks with error sources set at low, nominal, and high levels. Ground station measurements of the RSO are included using the nominal tasking profiles. The figures presented are of the RSO position for the entire 2 week period.

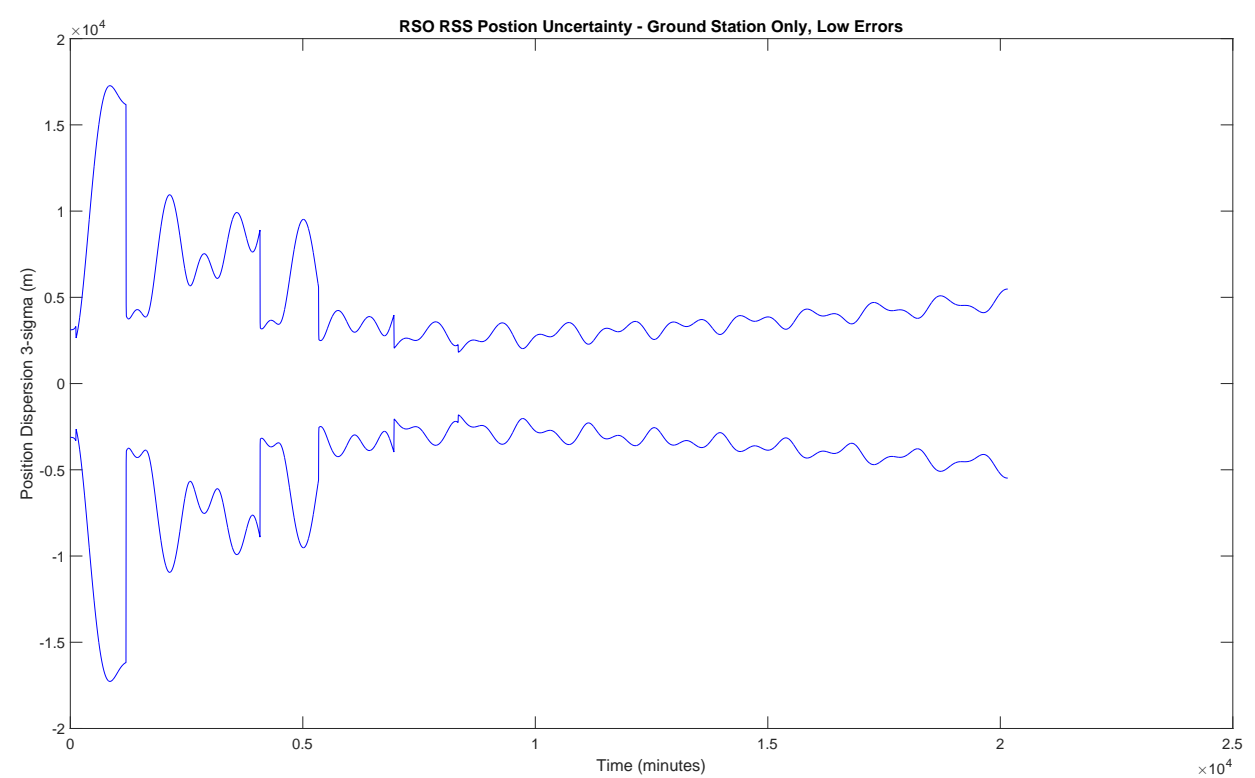

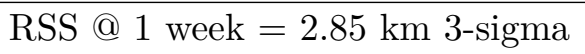

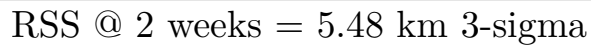

Fig. 10.1: Baseline Results - GS Only Measurements, Nominal GS Tasking Profile, Low Initial Errors 


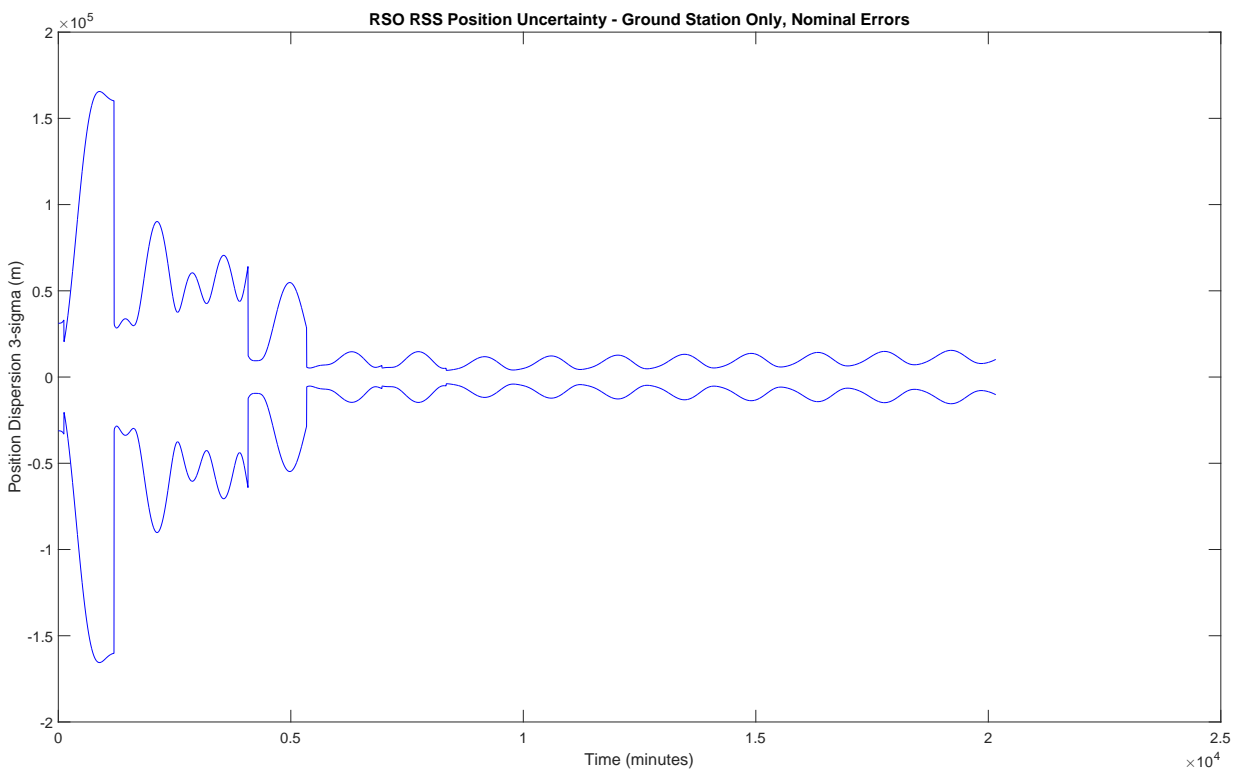

RSS @ 1 week $=5.66$ km 3-sigma

RSS @ 2 weeks $=10.23 \mathrm{~km} 3$-sigma

Fig. 10.2: Baseline Results - GS Only Measurements, Nominal GS Tasking Profile, Nominal Initial Errors 


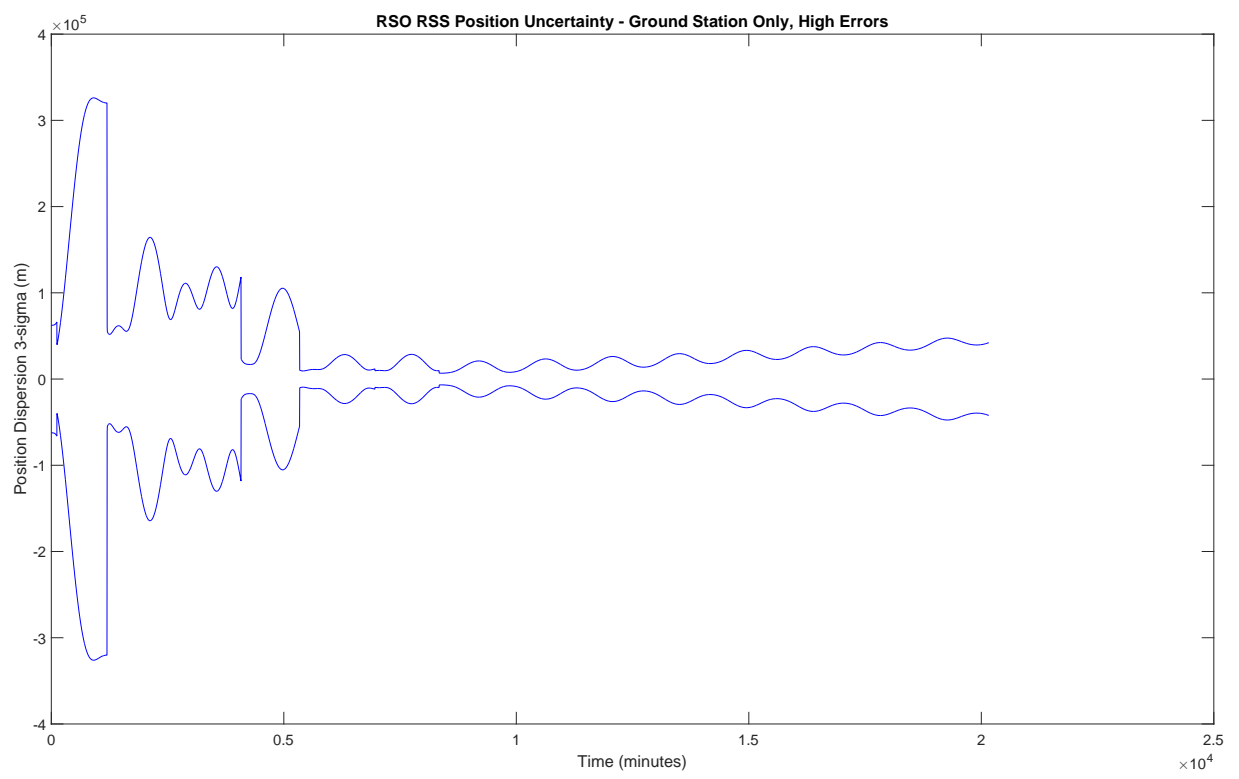

RSS @ 1 week $=9.95 \mathrm{~km} 3$-sigma $\quad$ RSS @ 2 weeks $=42.19 \mathrm{~km} 3$-sigma

Fig. 10.3: Baseline Results - GS Only Measurements, Nominal GS Tasking Profile, High Initial Errors

\subsection{Low Circular Space Sensor Orbit}

Presented in Figures 10.4 through 10.6 is the achievable RSO position accuracy at 1 week and 2 weeks with error sources set at low, nominal, and high levels. GPS is set at the nominal level for all scenarios, updating the space sensor position and velocity every 10 minutes. Ground station and low circular orbit space sensor measurements of the RSO are included using the nominal tasking profiles. The figures presented are of the RSO position for the entire 2 week period. 


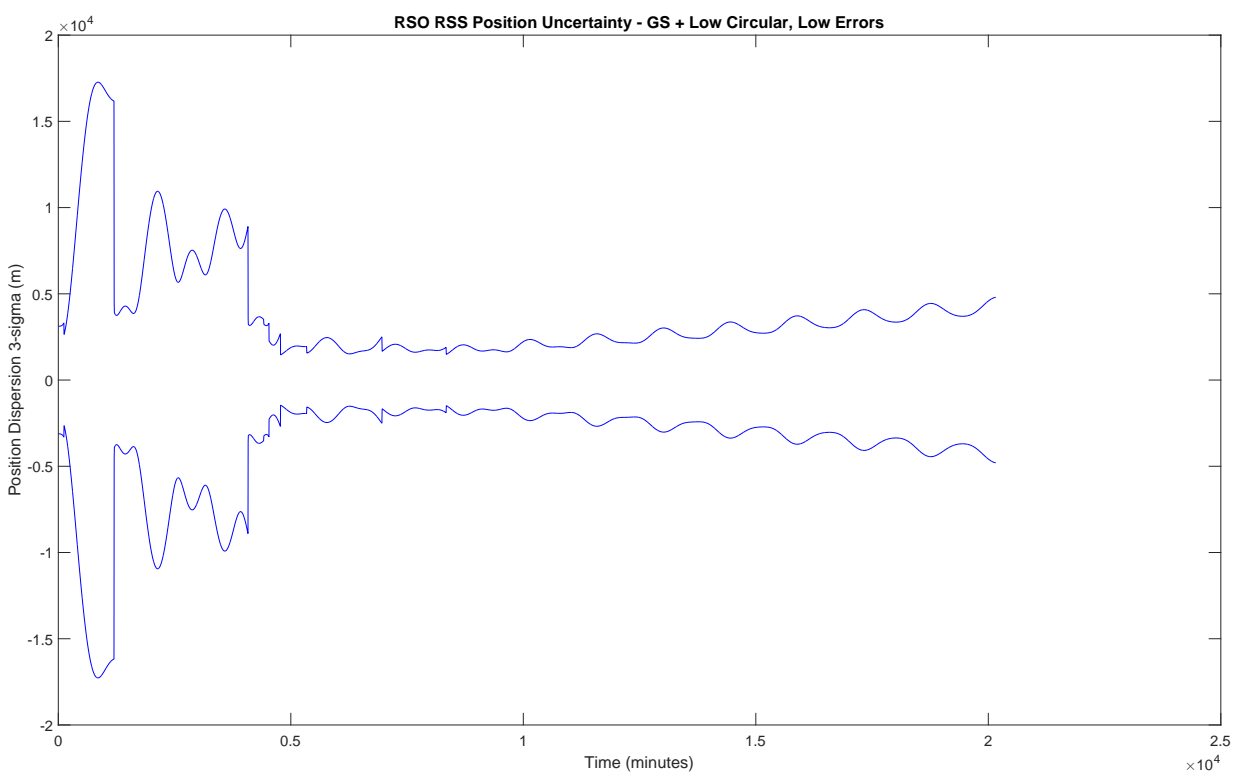

RSS @ 1 week $=2.33 \mathrm{~km} 3$-sigma

RSS @ 2 weeks $=4.80 \mathrm{~km} 3$-sigma

Fig. 10.4: Baseline Results - GS and Low Circular Orbit SS Measurements, Nominal Tasking Profiles, Low Initial Errors 


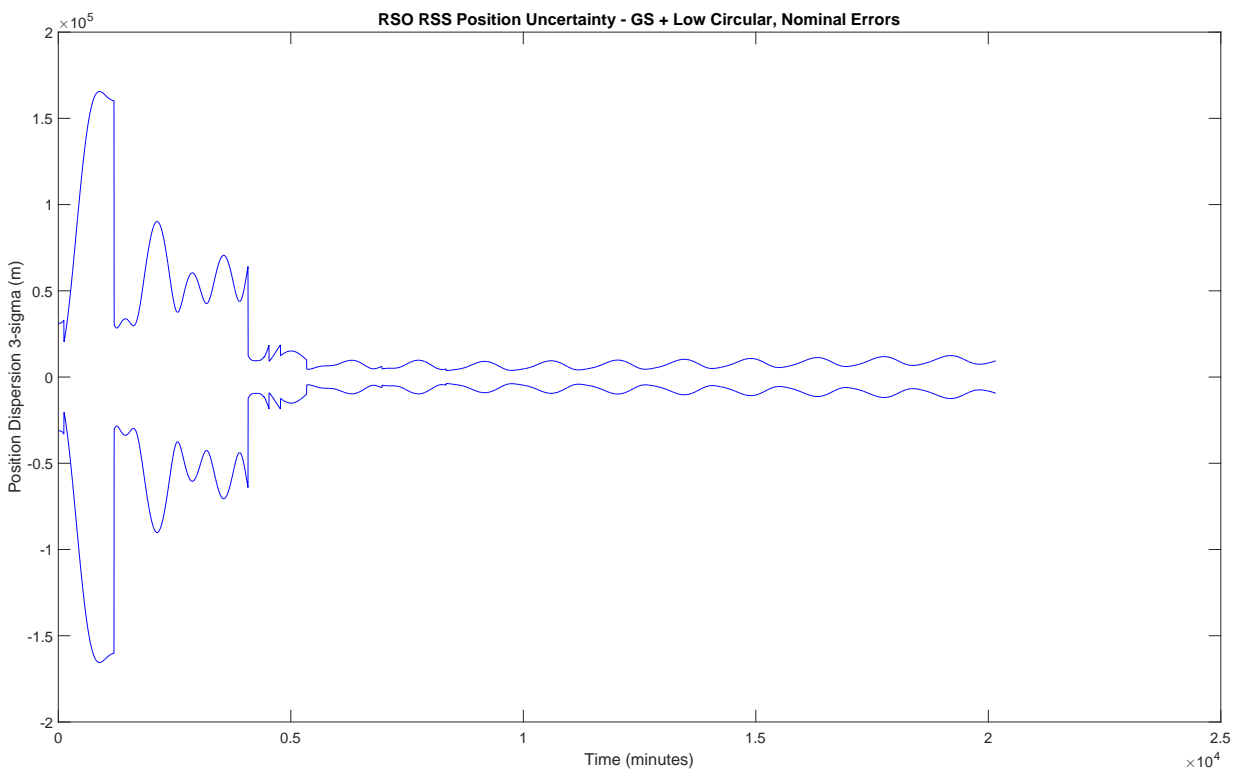

RSS @ 1 week $=5.14$ km 3-sigma

RSS@ 2 weeks $=9.38 \mathrm{~km} 3$-sigma

Fig. 10.5: Baseline Results - GS and Low Circular Orbit SS Measurements, Nominal Tasking Profiles, Nominal Initial Errors 


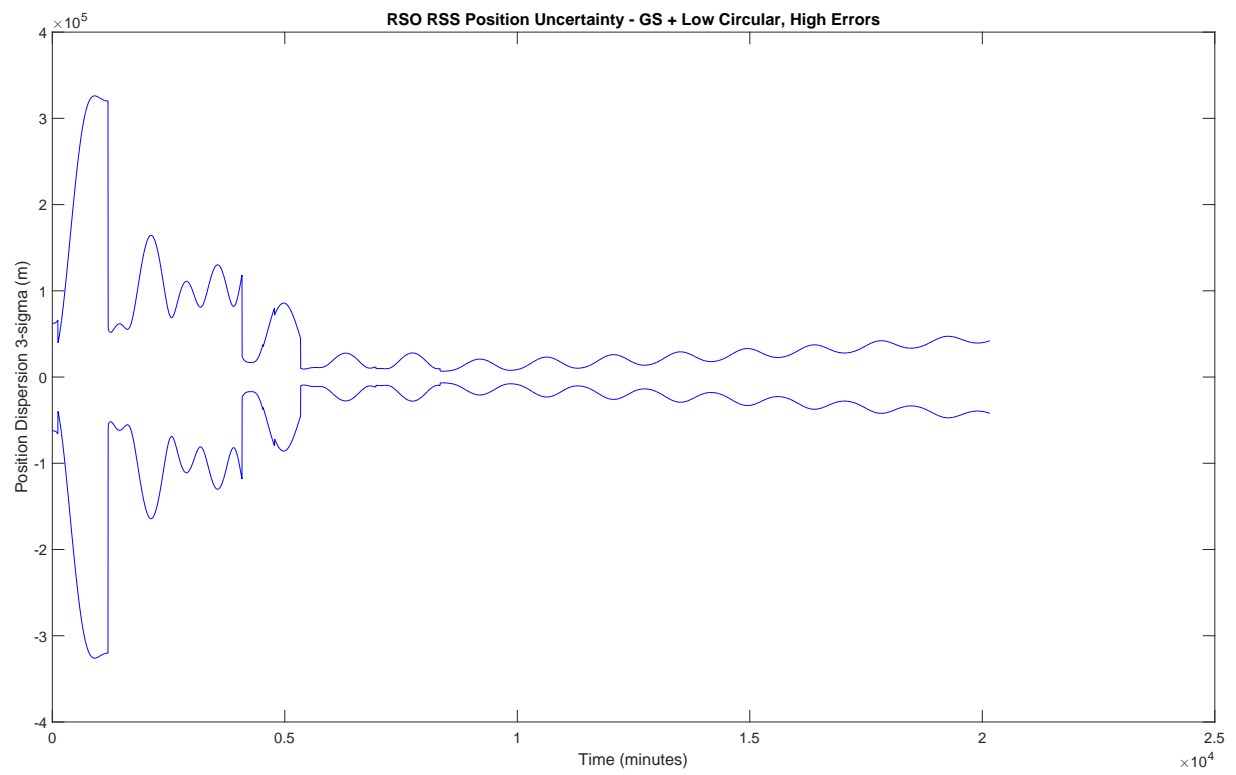

RSS @ 1 week $=9.90 \mathrm{~km} 3$-sigma RSS @ 2 weeks $=42.11 \mathrm{~km} 3$-sigma

Fig. 10.6: Baseline Results - GS and Low Circular Orbit SS Measurements, Nominal Tasking Profiles, High Initial Errors

\subsection{High Circular Space Sensor Orbit}

Presented in Figures 10.7 through 10.9 is the achievable RSO position accuracy at 1 week and 2 weeks with error sources set at low, nominal, and high levels. GPS is set at the nominal level for all scenarios, updating the space sensor position and velocity every 10 minutes. Ground station and high circular orbit space sensor measurements of the RSO are included using their nominal tasking profiles. The figures presented are of the RSO position for the entire 2 week period. 


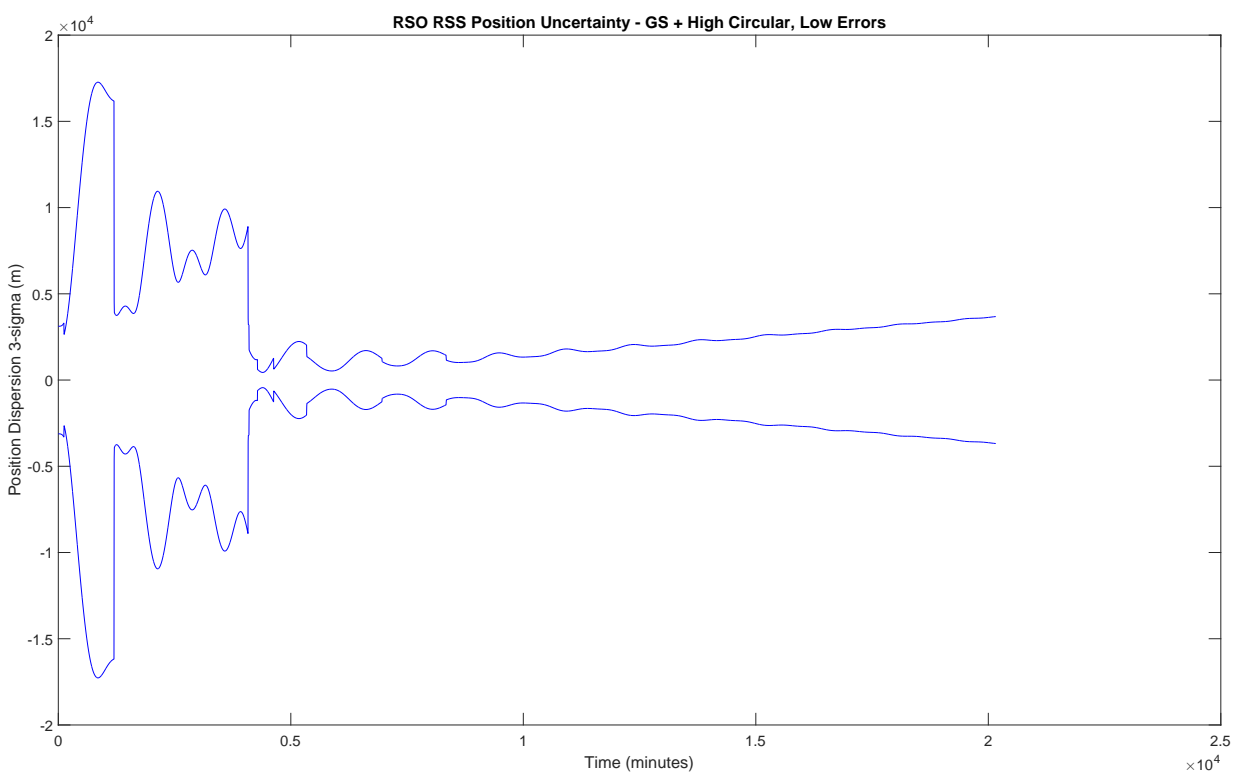

RSS @ 1 week = 1.34 km 3-sigma RSS @ 2 weeks $=3.68 \mathrm{~km} 3$-sigma

Fig. 10.7: Baseline Results - GS and High Circular Orbit SS Measurements, Nominal Tasking Profiles, Low Initial Errors 


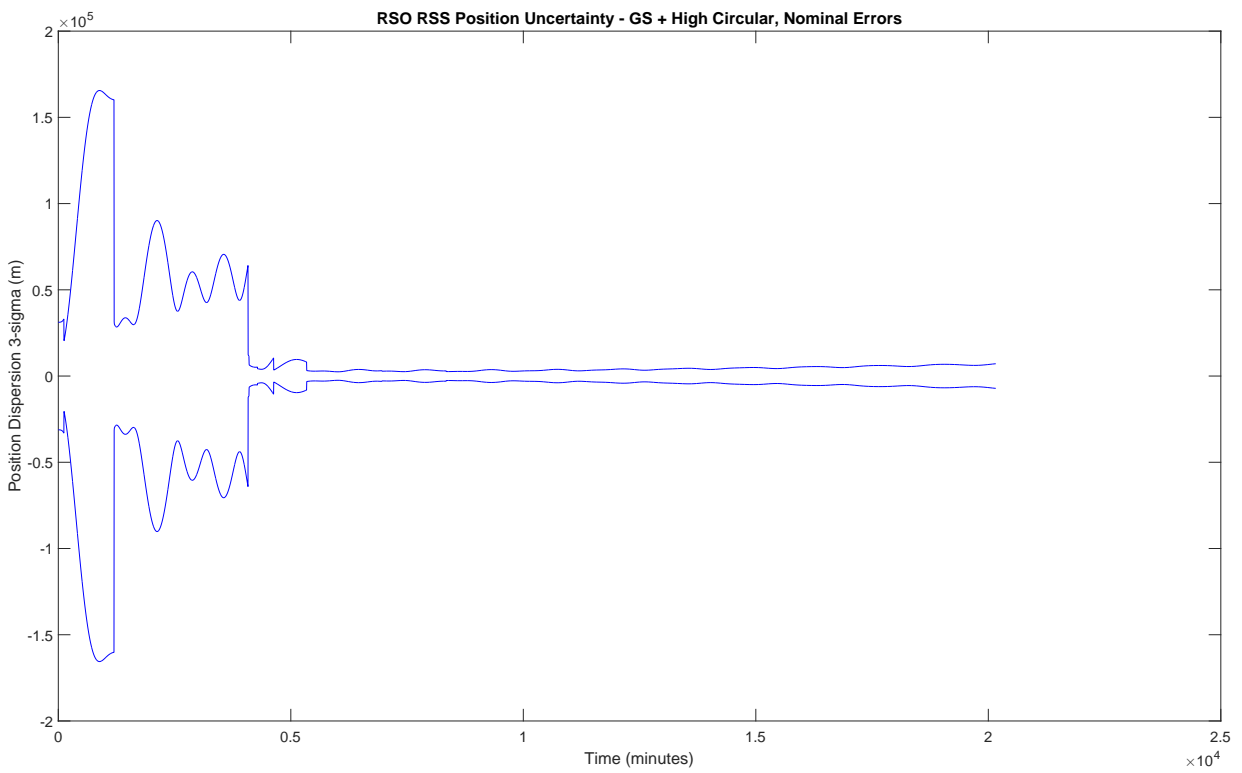

RSS @ 1 week = 3.03 km 3-sigma RSS @ 2 weeks $=7.14 \mathrm{~km} 3$-sigma

Fig. 10.8: Baseline Results - GS and High Circular Orbit SS Measurements, Nominal Tasking Profiles, Nominal Initial Errors 


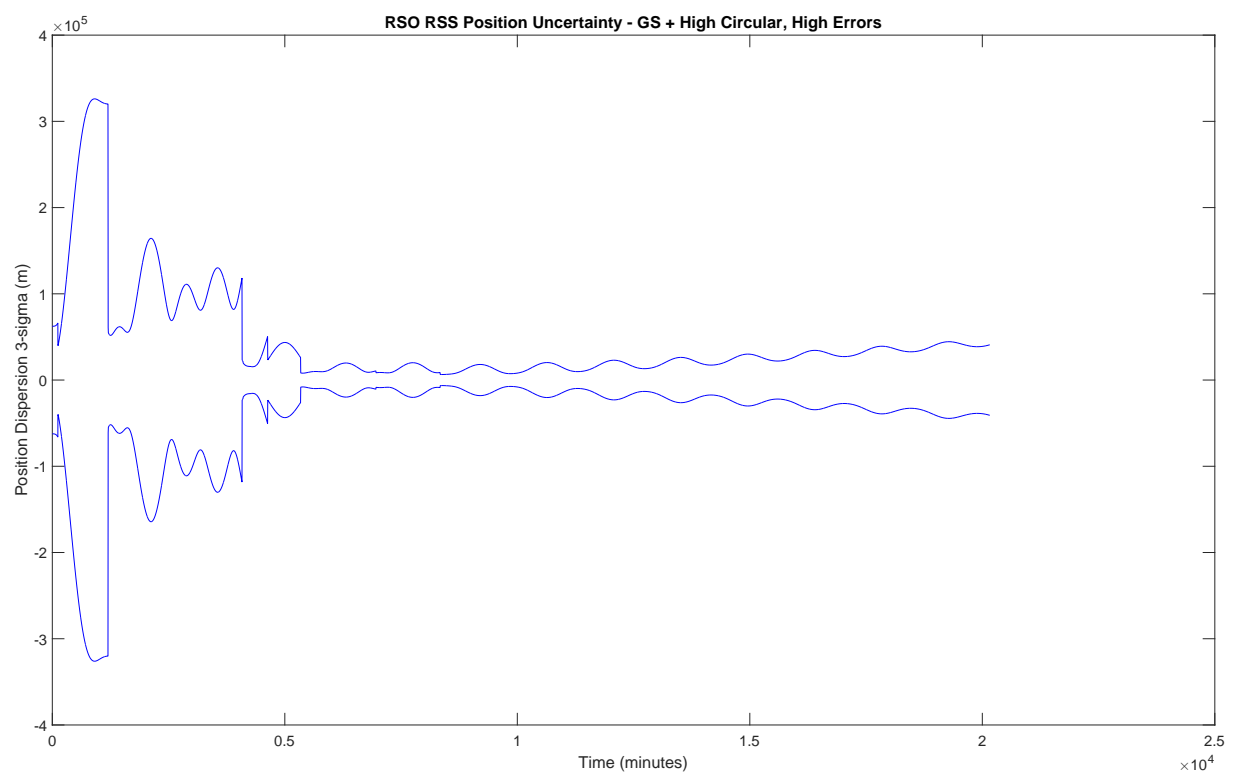

RSS @ 1 week $=8.98 \mathrm{~km} 3$-sigma $\quad$ RSS @ 2 weeks $=40.91 \mathrm{~km} 3$-sigma

Fig. 10.9: Baseline Results - GS and High Circular Orbit SS Measurements, Nominal Tasking Profiles, High Initial Errors

\subsection{Elliptical Space Sensor Orbit}

Presented in Figures 10.10 through 10.12 is the achievable RSO position accuracy at 1 week and 2 weeks with error sources set at low, nominal, and high levels. GPS is set at the nominal level for all scenarios, updating the space sensor position and velocity every 10 minutes. Ground station and elliptical orbit space sensor measurements of the RSO are included using their nominal tasking profiles. The figures presented are of the RSO position for the entire 2 week period. 


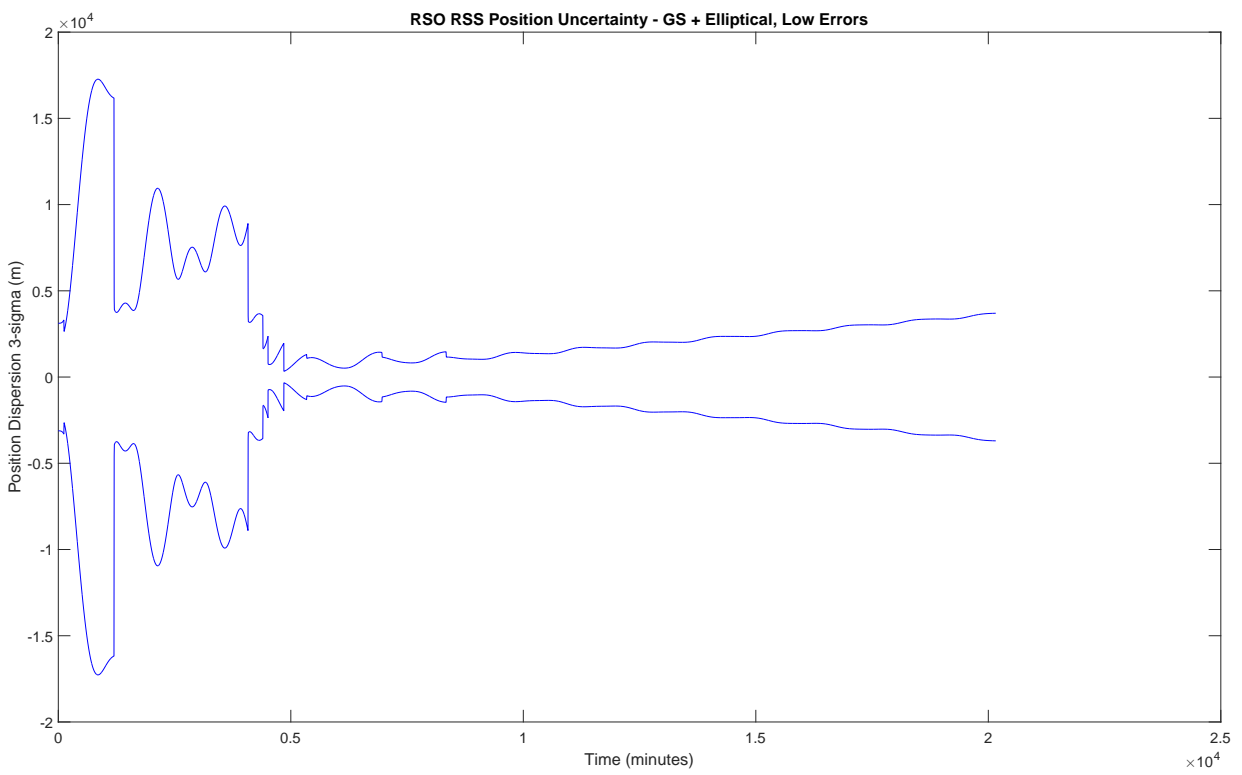

RSS @ 1 week $=1.38$ km 3-sigma

RSS @ 2 weeks $=3.70 \mathrm{~km} 3$-sigma

Fig. 10.10: Baseline Results - GS and Elliptical Orbit SS Measurements, Nominal Tasking Profiles, Low Initial Errors 


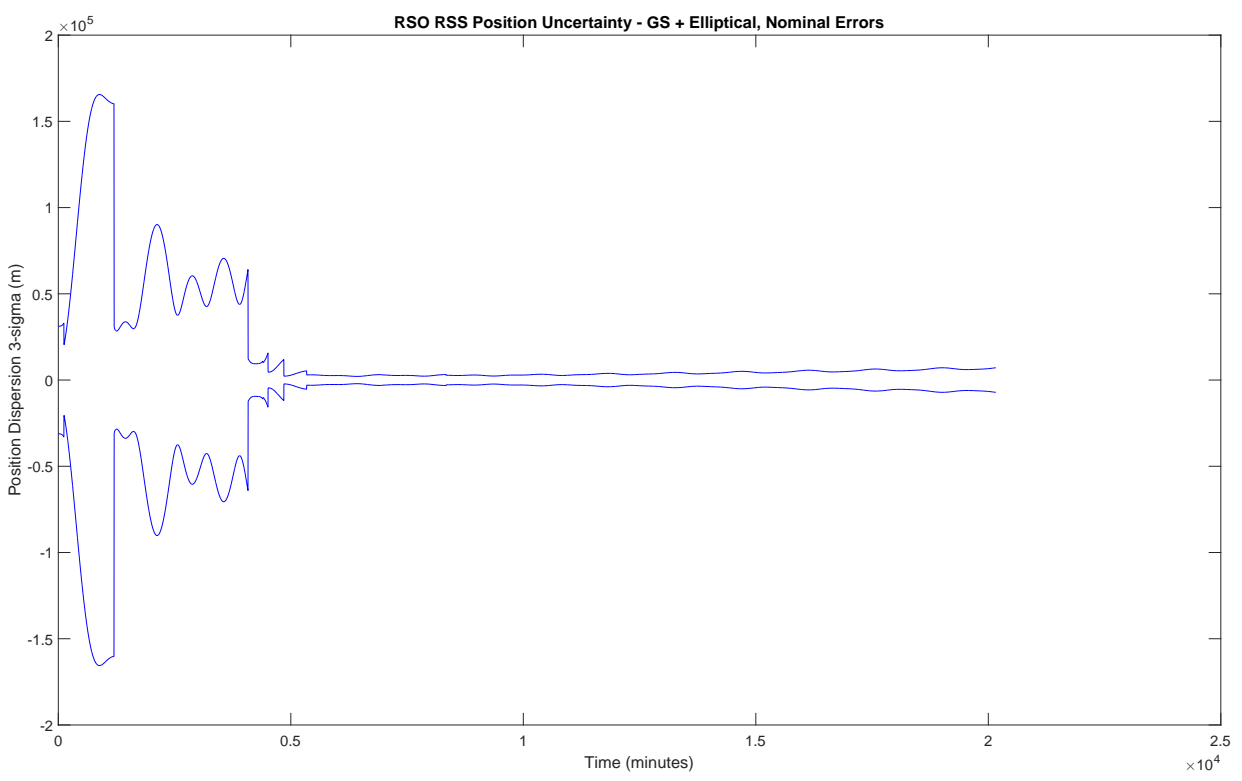

RSS @ 1 week $=2.96$ km 3-sigma

RSS@ 2 weeks $=7.14 \mathrm{~km} \mathrm{3-sigma}$

Fig. 10.11: Baseline Results - GS and Elliptical Orbit SS Measurements, Nominal Tasking Profiles, Nominal Initial Errors 


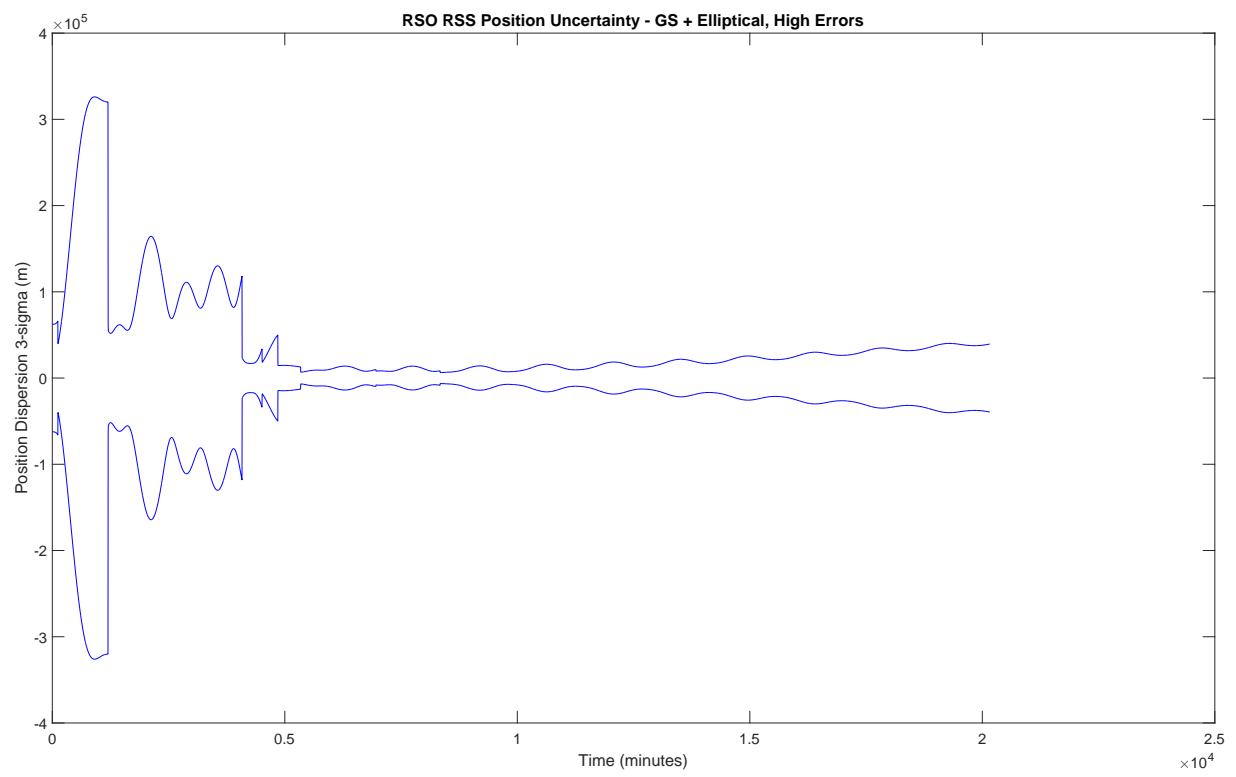

RSS @ 1 week $=8.55 \mathrm{~km} 3$-sigma RSS @ 2 weeks $=39.49 \mathrm{~km} 3$-sigma

Fig. 10.12: Baseline Results - GS and Elliptical Orbit SS Measurements, Nominal Tasking Profiles, High Initial Errors

\subsection{Summary}

Table 10.1 summarizes the results of the RSO position at the end of 1 week. Table 10.2 summarizes the results of the RSO position at the end of 2 weeks. The results clearly show that augmentation with the space sensor improves the ground station only results in all cases. Another take away from these results is that the low circular orbit space sensor results in the largest RSS error out of the three space sensor orbit results; this can be expected as the space sensor is furthest away from the RSO in this orbit. The high circular and elliptical space sensor orbit results are very comparable, and trade back and forth as to which is the best given the conditions of the scenario. 
Table 10.1: Baseline 1-week RSO Position Results, Nominal Tasking Profiles

\begin{tabular}{|c|c|c|c|c|}
\hline \multirow{2}{*}{ Error Levels } & \multicolumn{4}{|c|}{ 1-week 3-sigma (RSS) } \\
\cline { 2 - 5 } & GS Only & GS+Low Circ & GS+High Circ & GS+Elliptical \\
\hline Low & $2.85 \mathrm{~km}$ & $2.33 \mathrm{~km}$ & $1.34 \mathrm{~km}$ & $1.38 \mathrm{~km}$ \\
\hline Nominal & $5.66 \mathrm{~km}$ & $5.14 \mathrm{~km}$ & $3.03 \mathrm{~km}$ & $2.96 \mathrm{~km}$ \\
\hline High & $9.95 \mathrm{~km}$ & $9.90 \mathrm{~km}$ & $8.98 \mathrm{~km}$ & $8.55 \mathrm{~km}$ \\
\hline
\end{tabular}

Table 10.2: Baseline 2-week RSO Position Results, Nominal Tasking Profiles

\begin{tabular}{|c|c|c|c|c|}
\hline \multirow{2}{*}{ Error Levels } & \multicolumn{4}{|c|}{ 2-week 3-sigma (RSS) } \\
\cline { 2 - 5 } & GS Only & GS+Low Circ & GS+High Circ & GS+Elliptical \\
\hline Low & $5.48 \mathrm{~km}$ & $4.80 \mathrm{~km}$ & $3.68 \mathrm{~km}$ & $3.70 \mathrm{~km}$ \\
\hline Nominal & $10.23 \mathrm{~km}$ & $9.38 \mathrm{~km}$ & $7.14 \mathrm{~km}$ & $7.14 \mathrm{~km}$ \\
\hline High & $42.19 \mathrm{~km}$ & $42.11 \mathrm{~km}$ & $40.91 \mathrm{~km}$ & $39.49 \mathrm{~km}$ \\
\hline
\end{tabular}


CHAPTER 11

\section{RESULTS - REDUCED GROUND STATION MEASUREMENT PERIODS}

\subsection{Results Overview}

The goal of this chapter is to analyze the effect of reducing the amount of ground station measurement periods from the nominal amount of six down to two. The effect in the ground station measurement only scenario as well as the space sensor and ground station measurement scenarios will be analyzed. The ground station measurement periods are removed one by one starting with the removal of the last measurement period in the week.

\subsection{Ground Station Only}

Presented in Tables 11.1 and 11.2 is the achievable RSO position accuracy at 1 week and 2 weeks with error sources set at low, nominal, and high levels. The tables show results based on the nominal 6 ground station measurement periods (3 for each ground station) tasking scenario, down to 2 ground station measurement periods ( 1 for each ground station). 
Table 11.1: Number of GS Measurement Periods Analysis - Ground Station Only, 1 week results

\begin{tabular}{|c|c|c|c|}
\hline \multirow{2}{*}{ \# of GS Meas Periods } & \multicolumn{3}{|c|}{ 1-week 3-sigma Position RSS } \\
\cline { 2 - 4 } & Low Errors & Nominal Errors & High Errors \\
\hline \hline 6 (Baseline) & $2.85 \mathrm{~km}$ & $5.66 \mathrm{~km}$ & $9.95 \mathrm{~km}$ \\
\hline 5 & $3.52 \mathrm{~km}$ & $6.51 \mathrm{~km}$ & $12.06 \mathrm{~km}$ \\
\hline 4 & $7.05 \mathrm{~km}$ & $10.24 \mathrm{~km}$ & $19.01 \mathrm{~km}$ \\
\hline 3 & $7.27 \mathrm{~km}$ & $12.22 \mathrm{~km}$ & $22.41 \mathrm{~km}$ \\
\hline 2 & $24.68 \mathrm{~km}$ & $196.0 \mathrm{~km}$ & $362.64 \mathrm{~km}$ \\
\hline
\end{tabular}

Table 11.2: Number of GS Measurement Periods Analysis - Ground Station Only, 2 week results

\begin{tabular}{|c|c|c|c|}
\hline \multirow{2}{*}{ \# of GS Meas Periods } & \multicolumn{3}{|c|}{ 2-week 3-sigma Position RSS } \\
\cline { 2 - 4 } & Low Errors & Nominal Errors & High Errors \\
\hline \hline 6 (Baseline) & $5.48 \mathrm{~km}$ & $10.23 \mathrm{~km}$ & $42.19 \mathrm{~km}$ \\
\hline 5 & $6.93 \mathrm{~km}$ & $11.15 \mathrm{~km}$ & $43.93 \mathrm{~km}$ \\
\hline 4 & $13.78 \mathrm{~km}$ & $19.25 \mathrm{~km}$ & $57.36 \mathrm{~km}$ \\
\hline 3 & $13.97 \mathrm{~km}$ & $20.37 \mathrm{~km}$ & $58.68 \mathrm{~km}$ \\
\hline 2 & $48.72 \mathrm{~km}$ & $384.73 \mathrm{~km}$ & $714.46 \mathrm{~km}$ \\
\hline
\end{tabular}




\subsection{Low Circular Space Sensor Orbit}

Presented in Tables 11.3 and 11.4 is the achievable RSO position accuracy at 1 week and 2 weeks with error sources set at low, nominal, and high levels. GPS is set at the nominal level for all scenarios, updating the space sensor position and velocity every 10 minutes. Ground station and low circular space sensor measurements of the RSO are included. The low circular orbit space sensor measurement profile remains nominal for all results. The tables show results based on the nominal 6 ground station measurement periods tasking scenario, down to 2 ground station measurement periods.

Table 11.3: Number of GS Measurement Periods Analysis - GS and Low Circular SS, 1 week results

\begin{tabular}{|c|c|c|c|}
\hline \multirow{2}{*}{ \# of GS Meas Periods } & \multicolumn{3}{|c|}{ 1-week 3-sigma Position RSS } \\
\cline { 2 - 4 } & Low Errors & Nominal Errors & High Errors \\
\hline \hline 6 (Baseline) & $2.33 \mathrm{~km}$ & $5.14 \mathrm{~km}$ & $9.90 \mathrm{~km}$ \\
\hline 5 & $2.87 \mathrm{~km}$ & $5.97 \mathrm{~km}$ & $11.98 \mathrm{~km}$ \\
\hline 4 & $4.29 \mathrm{~km}$ & $9.77 \mathrm{~km}$ & $18.96 \mathrm{~km}$ \\
\hline 3 & $4.56 \mathrm{~km}$ & $9.91 \mathrm{~km}$ & $21.13 \mathrm{~km}$ \\
\hline 2 & $6.33 \mathrm{~km}$ & $57.87 \mathrm{~km}$ & $310.34 \mathrm{~km}$ \\
\hline
\end{tabular}


Table 11.4: Number of GS Measurement Periods Analysis - GS and Low Circular SS, 2 week results

\begin{tabular}{|c|c|c|c|}
\hline \multirow{2}{*}{ \# of GS Meas Periods } & \multicolumn{3}{|c|}{ 2-week 3-sigma Position RSS } \\
\cline { 2 - 4 } & Low Errors & Nominal Errors & High Errors \\
\hline \hline 6 (Baseline) & $4.80 \mathrm{~km}$ & $9.38 \mathrm{~km}$ & $42.11 \mathrm{~km}$ \\
\hline 5 & $5.98 \mathrm{~km}$ & $10.70 \mathrm{~km}$ & $43.90 \mathrm{~km}$ \\
\hline 4 & $8.83 \mathrm{~km}$ & $18.77 \mathrm{~km}$ & $57.34 \mathrm{~km}$ \\
\hline 3 & $9.44 \mathrm{~km}$ & $18.82 \mathrm{~km}$ & $58.14 \mathrm{~km}$ \\
\hline 2 & $12.81 \mathrm{~km}$ & $114.15 \mathrm{~km}$ & $612.02 \mathrm{~km}$ \\
\hline
\end{tabular}

\subsection{High Circular Space Sensor Orbit}

Presented in Tables 11.5 and 11.6 is the achievable RSO position accuracy at 1 week and 2 weeks with error sources set at low, nominal, and high levels. GPS is set at the nominal level for all scenarios, updating the space sensor position and velocity every 10 minutes. Ground station and high circular space sensor measurements of the RSO are included. The high circular orbit space sensor measurement profile remains nominal for all results. The tables show results based on the nominal 6 ground station measurement periods tasking scenario, down to 2 ground station measurement periods. 
Table 11.5: Number of GS Measurement Periods Analysis - GS and High Circular SS, 1 week results

\begin{tabular}{|c|c|c|c|}
\hline \multirow{2}{*}{ \# of GS Meas Periods } & \multicolumn{3}{|c|}{ 1-week 3-sigma Position RSS } \\
\cline { 2 - 4 } & Low Errors & Nominal Errors & High Errors \\
\hline \hline 6 (Baseline) & $1.34 \mathrm{~km}$ & $3.03 \mathrm{~km}$ & $8.98 \mathrm{~km}$ \\
\hline 5 & $1.56 \mathrm{~km}$ & $3.72 \mathrm{~km}$ & $10.91 \mathrm{~km}$ \\
\hline 4 & $1.75 \mathrm{~km}$ & $5.05 \mathrm{~km}$ & $18.10 \mathrm{~km}$ \\
\hline 3 & $2.00 \mathrm{~km}$ & $5.59 \mathrm{~km}$ & $18.60 \mathrm{~km}$ \\
\hline 2 & $2.13 \mathrm{~km}$ & $8.27 \mathrm{~km}$ & $52.74 \mathrm{~km}$ \\
\hline
\end{tabular}

Table 11.6: Number of GS Measurement Periods Analysis - GS and High Circular SS, 2 week results

\begin{tabular}{|c|c|c|c|}
\hline \multirow{2}{*}{ \# of GS Meas Periods } & \multicolumn{3}{|c|}{ 2-week 3-sigma Position RSS } \\
\cline { 2 - 4 } & Low Errors & Nominal Errors & High Errors \\
\hline \hline 6 (Baseline) & $3.68 \mathrm{~km}$ & $7.14 \mathrm{~km}$ & $40.91 \mathrm{~km}$ \\
\hline 5 & $4.22 \mathrm{~km}$ & $8.65 \mathrm{~km}$ & $43.58 \mathrm{~km}$ \\
\hline 4 & $4.68 \mathrm{~km}$ & $11.92 \mathrm{~km}$ & $56.90 \mathrm{~km}$ \\
\hline 3 & $5.22 \mathrm{~km}$ & $13.14 \mathrm{~km}$ & $57.03 \mathrm{~km}$ \\
\hline 2 & $5.47 \mathrm{~km}$ & $19.28 \mathrm{~km}$ & $113.75 \mathrm{~km}$ \\
\hline
\end{tabular}




\subsection{Elliptical Space Sensor Orbit}

Presented in Tables 11.7 and 11.8 is the achievable RSO position accuracy at 1 week and 2 weeks with error sources set at low, nominal, and high levels. GPS is set at the nominal level for all scenarios, updating the space sensor position and velocity every 10 minutes. Ground station and elliptical space sensor measurements of the RSO are included. The elliptical orbit space sensor measurement profile remains nominal for all results. The tables show results based on the nominal 6 ground station measurement periods tasking scenario, down to 2 ground station measurement periods.

Table 11.7: Number of GS Measurement Periods Analysis - GS and Elliptical SS, 1 week results

\begin{tabular}{|c|c|c|c|}
\hline \multirow{2}{*}{ \# of GS Meas Periods } & \multicolumn{3}{|c|}{ 1-week 3-sigma Position RSS } \\
\cline { 2 - 4 } & Low Errors & Nominal Errors & High Errors \\
\hline \hline 6 (Baseline) & $1.38 \mathrm{~km}$ & $2.96 \mathrm{~km}$ & $8.55 \mathrm{~km}$ \\
\hline 5 & $1.69 \mathrm{~km}$ & $3.47 \mathrm{~km}$ & $10.56 \mathrm{~km}$ \\
\hline 4 & $2.02 \mathrm{~km}$ & $4.36 \mathrm{~km}$ & $17.67 \mathrm{~km}$ \\
\hline 3 & $2.28 \mathrm{~km}$ & $6.28 \mathrm{~km}$ & $17.92 \mathrm{~km}$ \\
\hline 2 & $2.47 \mathrm{~km}$ & $10.85 \mathrm{~km}$ & $55.54 \mathrm{~km}$ \\
\hline
\end{tabular}


Table 11.8: Number of GS Measurement Periods Analysis - GS and Elliptical SS, 2 week results

\begin{tabular}{|c|c|c|c|}
\hline \multirow{2}{*}{ \# of GS Meas Periods } & \multicolumn{3}{|c|}{ 2-week 3-sigma Position RSS } \\
\cline { 2 - 4 } & Low Errors & Nominal Errors & High Errors \\
\hline \hline 6 (Baseline) & $3.70 \mathrm{~km}$ & $7.14 \mathrm{~km}$ & $39.49 \mathrm{~km}$ \\
\hline 5 & $4.39 \mathrm{~km}$ & $8.27 \mathrm{~km}$ & $43.44 \mathrm{~km}$ \\
\hline 4 & $5.09 \mathrm{~km}$ & $10.61 \mathrm{~km}$ & $56.61 \mathrm{~km}$ \\
\hline 3 & $5.58 \mathrm{~km}$ & $13.99 \mathrm{~km}$ & $56.90 \mathrm{~km}$ \\
\hline 2 & $5.89 \mathrm{~km}$ & $23.60 \mathrm{~km}$ & $121.16 \mathrm{~km}$ \\
\hline
\end{tabular}

\subsection{Summary}

As one might expect, it can be seen that in each scenario as the number of ground station measurement periods reduces from 6 periods to 2 periods, the position RSS values at both the 1 week and 2 week marks is larger. Another take away is that, for a given scenario, the ground station only position RSS error results are always larger than the ground station with space sensor augmentation results. The low circular orbit space sensor augmentation results remain the largest position RSS error out of the three space sensor orbits, as was seen in Chapter 10. The high circular space sensor and elliptical space sensor augmentation results are very similar and depending on the scenario trade back and forth the smallest position RSS error results.

When only two ground station measurement periods are performed in addition to the space sensor measurements, the results are larger position RSS errors (in some cases $50 \%+$ larger) when compared to when there are three ground station measurement periods in addition to the space sensor measurements. Recalling the ground station and space sensor 
measurement tasking profiles presented in Chapter 3, there is not much time between the third ground station measurement period and the first of three space sensor measurement periods for all space sensor orbits. This means the errors are already reduced going into the first space sensor measurement period when there are three ground station measurement periods. When only two ground station measurement periods are performed, the errors have time to grow before the first space sensor measurement period is performed. Therefore, even with the measurements from the space sensor the errors grow to much larger values over 1 or 2 weeks. 


\section{CHAPTER 12 \\ RESULTS - EXTENDED SPACE SENSOR MEASUREMENT PERIODS}

\subsection{Results Overview}

The goal of this chapter is to analyze the effect of extending the length of the space sensor measurement periods from 5 minutes to 20 minutes. The effect of the extended space sensor measurements is studied across all space sensor orbits: low circular, high circular, and elliptical. The space sensor measurement profile is kept nominal throughout all scenarios, the measurement periods begin at the same time as when the measurement periods were 5 minutes, but now they are carried out for 20 minutes. Ground station measurements are used in the analysis. The effect of having reduced ground station measurements is studied as well, which places more emphasis on the extended space sensor measurements. The ground station measurement periods are removed one by one starting with the removal of the last measurement period in the week.

\subsection{Low Circular Space Sensor Orbit}

Table 12.1 summarizes the extended space sensor measurement period results for each error level: low error level, nominal error level, and high error level for the low circular orbit space sensor. The percent change in RSO position accuracy, when compared to the results presented in the previous chapter, is given at 1 week and 2 weeks based on the extended space sensor measurements as well as reducing the ground station measurement periods. GPS is set at the nominal level for all scenarios, updating the space sensor position and velocity every 10 minutes. 
Table 12.1: Extended SS Measurement Periods Analysis - GS and Low Circular SS

\begin{tabular}{|c|c|c|c|c|}
\hline \# of GS & & Low Errors & Nominal Errors & High Errors \\
\hline Meas Periods & & \multicolumn{3}{|c|}{$\%$ Change } \\
\hline \multirow{2}{*}{6} & 1 week & $-18.6 \%$ & $-8.9 \%$ & $-1 \%$ \\
\hline & 2 week & $-11.4 \%$ & $-6.8 \%$ & $-0.3 \%$ \\
\hline \multirow{2}{*}{5} & 1 week & $-19.9 \%$ & $-6.7 \%$ & $-1.4 \%$ \\
\hline & 2 week & $-13.9 \%$ & $-2.95 \%$ & $-0.1 \%$ \\
\hline \multirow{2}{*}{4} & 1 week & $-30.6 \%$ & $-6.4 \%$ & $-0.6 \%$ \\
\hline & 2 week & $-25.7 \%$ & $-4.5 \%$ & $-0.1 \%$ \\
\hline \multirow{2}{*}{3} & 1 week & $-28.4 \%$ & $-6.8 \%$ & $-5.7 \%$ \\
\hline & 2 week & $-24.01 \%$ & $-4.2 \%$ & $-0.8 \%$ \\
\hline \multirow{2}{*}{2} & 1 week & $-39 \%$ & $-46 \%$ & $-25 \%$ \\
\hline & 2 week & $-35.5 \%$ & $-45.4 \%$ & $-24.7 \%$ \\
\hline
\end{tabular}

\subsection{High Circular Space Sensor Orbit}

Table 12.2 summarizes the extended space sensor measurement period results for each error level: low error level, nominal error level, and high error level for the high circular orbit space sensor. The percent change in RSO position accuracy, when compared to the results presented in the previous chapter, is given at 1 week and 2 weeks based on the extended space sensor measurements as well as reducing the ground station measurement periods. GPS is set at the nominal level for all scenarios, updating the space sensor position and velocity every 10 minutes. 
Table 12.2: Extended SS Measurement Periods Analysis - GS and High Circular SS

\begin{tabular}{|c|c|c|c|c|}
\hline \# of GS & & Low Errors & Nominal Errors & High Errors \\
\hline Meas Periods & & \multicolumn{3}{|c|}{$\%$ Change } \\
\hline \multirow{2}{*}{6} & 1 week & $-2.7 \%$ & $-21.6 \%$ & $-13.5 \%$ \\
\hline & 2 week & $-0.8 \%$ & $-4.6 \%$ & $-3.6 \%$ \\
\hline \multirow{2}{*}{5} & 1 week & $-2.9 \%$ & $-22.1 \%$ & $-8.4 \%$ \\
\hline & 2 week & $-1.1 \%$ & $-6.7 \%$ & $-0.5 \%$ \\
\hline \multirow{2}{*}{4} & 1 week & $-3.6 \%$ & $-24.3 \%$ & $-5.5 \%$ \\
\hline & 2 week & $-1.8 \%$ & $-11.9 \%$ & $-1.1 \%$ \\
\hline \multirow{2}{*}{3} & 1 week & $-3.6 \%$ & $-14.2 \%$ & $-6.9 \%$ \\
\hline & 2 week & $-2.1 \%$ & $-6.3 \%$ & $-1.02 \%$ \\
\hline \multirow{2}{*}{2} & 1 week & $-4.5 \%$ & $-17.3 \%$ & $-40 \%$ \\
\hline & 2 week & $-2.8 \%$ & $-11.4 \%$ & $-30.4 \%$ \\
\hline
\end{tabular}

\subsection{Elliptical Space Sensor Orbit}

Table 12.3 summarizes the extended space sensor measurement period results for each error level: low error level, nominal error level, and high error level for the elliptical orbit space sensor. The percent change in RSO position accuracy, when compared to the results presented in the previous chapter, is given at 1 week and 2 weeks based on the extended space sensor measurements as well as reducing the ground station measurement periods. GPS is set at the nominal level for all scenarios, updating the space sensor position and velocity every 10 minutes. 
Table 12.3: Extended SS Measurement Periods Analysis - GS and Elliptical SS

\begin{tabular}{|c|c|c|c|c|}
\hline \# of GS & & Low Errors & Nominal Errors & High Errors \\
\hline Meas Periods & & \multicolumn{3}{|c|}{$\%$ Change } \\
\hline \multirow{2}{*}{6} & 1 week & $-1.07 \%$ & $-22.1 \%$ & $-10.6 \%$ \\
\hline & 2 week & $-0.4 \%$ & $-7.4 \%$ & $-2.99 \%$ \\
\hline \multirow{2}{*}{5} & 1 week & $-0.7 \%$ & $-19.4 \%$ & $-6.3 \%$ \\
\hline & 2 week & $-0.3 \%$ & $-7.6 \%$ & $-0.3 \%$ \\
\hline \multirow{2}{*}{4} & 1 week & $-0.5 \%$ & $-22.7 \%$ & $-6.3 \%$ \\
\hline & 2 week & $-0.2 \%$ & $-12.3 \%$ & $-1.5 \%$ \\
\hline \multirow{2}{*}{3} & 1 week & $-0.4 \%$ & $-4.8 \%$ & $-4.2 \%$ \\
\hline & 2 week & $-0.2 \%$ & $-2.7 \%$ & $-0.7 \%$ \\
\hline \multirow{2}{*}{2} & 1 week & $-0.4 \%$ & $-5.5 \%$ & $-36.6 \%$ \\
\hline & 2 week & $-0.2 \%$ & $-4.4 \%$ & $-29.4 \%$ \\
\hline
\end{tabular}

\subsection{Summary}

The main takeaway from the results presented above is extending the space sensor measurement periods to 20 minutes improves the RSO position accuracy in all scenarios, as one might expect. Another important observation is that the extended measurement periods tend to be more helpful for the low circular orbit, especially when there are low errors. When there are two ground station measurement periods, the extended space sensor measurement periods are very helpful. The effect of the extended space sensor measurement periods diminishes over time, as seen with the 2 week results having a less percent change 
than the 1 week results in all scenarios. 


\section{CHAPTER 13 \\ RESULTS - RSO INCLINATION ANALYSIS}

\subsection{Results Overview}

This chapter analyzes the effect of inclining the RSO to a 15 degree inclination. Geosynchronous satellites that are no longer under active control begin to drift, and can reach an inclination of 15 degrees over several decades. Some of the first geosynchronous satellites launched are now at inclinations of 15 degrees [6]. The goal of this chapter is to see if the orbit estimation results are as accurate when tracking an inclined RSO versus the nominal RSO. Ground station measurements alone are analyzed, as well as ground station measurements and three different space sensor orbits, low circular, high circular, and elliptical measurements.

\subsection{Ground Station Only}

Below are the results for the ground station only scenarios analyzing the change in RSO position accuracy when the RSO orbit is inclined to 15 degrees. Table 13.1 presents results for each of the three error levels: low error level, nominal error level, and high error level. The table gives percent change results for the 15 degree inclined RSO orbit when compared to the nominal 0.05 degree inclined RSO orbit. The results are provided after each measurement is taken during the simulation as well as at the 1 week and 2 week points. 
Table 13.1: RSO Inclination Analysis - Ground Station Only

\begin{tabular}{|c|c|c|c|}
\hline \multirow{2}{*}{ Point in } & Low Errors & Nominal Errors & High Errors \\
\cline { 2 - 4 } Simulation & \multicolumn{2}{|c|}{$\%$ Change - Position RSS } \\
\hline \hline 1st GS Meas* & $-0.8 \%$ & $-1.2 \%$ & $-1.3 \%$ \\
\hline 2nd GS Meas* & $-0.7 \%$ & $-1.1 \%$ & $-1.3 \%$ \\
\hline 3rd GS Meas* & $-0.8 \%$ & $+0.7 \%$ & $+0.7 \%$ \\
\hline 4th GS Meas & $-1.4 \%$ & $-1.1 \%$ & $-1.0 \%$ \\
\hline 5th GS Meas & $-2.2 \%$ & $-0.9 \%$ & $-0.7 \%$ \\
\hline 6th GS Meas & $-2.2 \%$ & $-1.1 \%$ & $-1.0 \%$ \\
\hline 1 week & $-2.6 \%$ & $-2.0 \%$ & $-2.1 \%$ \\
\hline 2 week & $-2.4 \%$ & $-1.5 \%$ & $-0.4 \%$ \\
\hline
\end{tabular}

* - These three rows remain the same for all ground station and space sensor measurement analysis results presented below, due to the fact that space sensor measurements are not introduced until after these measurements.

\subsection{Low Circular Space Sensor}

Setting the RSO inclination to 15 degrees changed the relative motion range between the RSO and the low circular space sensor slightly for all space sensor orbits. Figure 13.1 demonstrates the change in relative motion for the low circular space sensor orbit. The nominal space sensor tracking schedule was used for this analysis, however the relative range for these measurements will be approximately the same as in the 0.05 degree inclined RSO analysis. 


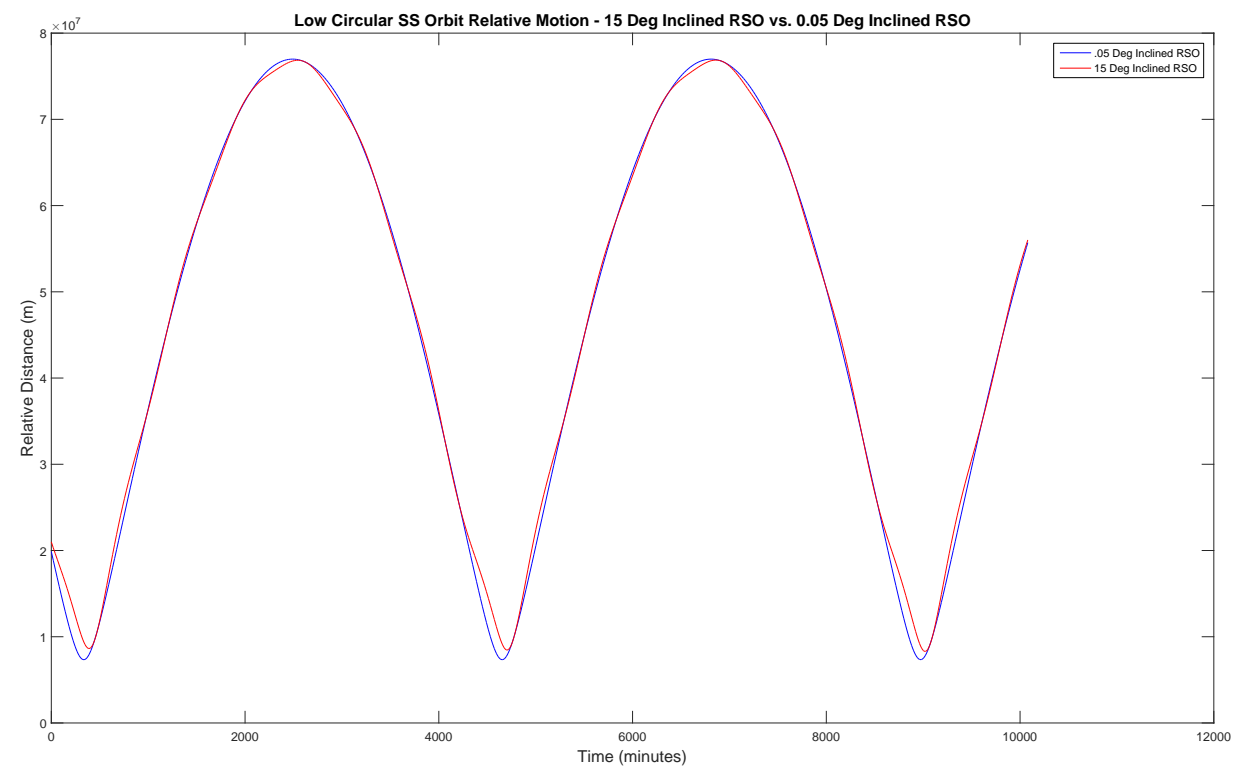

Fig. 13.1: Low Circular Space Sensor Relative Motion - RSO Inclined Analysis

Below are the results for the low circular orbit space sensor and ground station measurement scenarios analyzing the change in RSO position accuracy when the RSO orbit is inclined to 15 degrees. Table 13.2 present results for each of the three error levels: low error level, nominal error level, and high error level. The table gives percent change results for the 15 degree inclined RSO orbit when compared to the nominal 0.05 degree inclined RSO orbit. The results are provided after each measurement is taken during the simulation as well as at the 1 week and 2 week points. 
Table 13.2: RSO Inclination Analysis - GS and Low Circular SS

\begin{tabular}{|c|c|c|c|}
\hline \multirow{2}{*}{ Point in } & Low Errors & Nominal Errors & High Errors \\
\cline { 2 - 4 } Simulation & \multicolumn{2}{|c|}{$\%$ Change - Position RSS } \\
\hline \hline 1st SS Meas & $-10.1 \%$ & $-0.6 \%$ & $-0.03 \%$ \\
\hline 2nd SS Meas & $-15.4 \%$ & $-1.5 \%$ & $-0.05 \%$ \\
\hline 3rd SS Meas & $-5.1 \%$ & $-4.7 \%$ & $-1.9 \%$ \\
\hline 4th GS Meas & $-7.7 \%$ & $-3.0 \%$ & $-1.1 \%$ \\
\hline 5th GS Meas & $-10.6 \%$ & $-2.96 \%$ & $-0.8 \%$ \\
\hline 6th GS Meas & $-7.4 \%$ & $-2.1 \%$ & $-1.0 \%$ \\
\hline 1 week & $-11.4 \%$ & $-2.2 \%$ & $-2.1 \%$ \\
\hline 2 week & $-7.9 \%$ & $-1.4 \%$ & $-0.4 \%$ \\
\hline
\end{tabular}

\subsection{High Circular Space Sensor}

Figure 13.2 demonstrates the change in relative motion for the high circular space sensor orbit. The nominal space sensor tracking schedule was used for this analysis, however the relative range for these measurements will be potentially much different than in the 0.05 degree inclined RSO analysis. The 15 degree inclined RSO introduced an oscillatory relative motion when compared to the 0.05 degree inclination RSO. As a result of this very different relative motion, the results of this analysis for the high circular space sensor orbit vary back and forth between a positive and negative change, depending on when the space sensor measurements are taken. Figure 13.3 is an overall plot of the RSO RSS 3-sigma position for the nominal errors, for the entire 2 week simulation. Figure 13.4 zooms in on the space sensor measurement portion of the two weeks, it can be seen that the inclined and 
not inclined position RSS curves vary back and forth with each other. Figure 13.5 zooms in on the second week of the simulation, which demonstrates that at one week the 15 degree inclined RSO has worse results and at the end of two weeks the 0.05 degree inclined RSO has worse results. It depends on the point in time when the data is extracted from the plots as to which position RSS produces a lower result.

The percent change results are presented in Table 13.3. This same variation is seen for the low error level results. For the high error level results, the oscillatory motion of the results do not cross each other overtime and therefore the results remain consistent with the 15 degree inclined RSO producing better results.

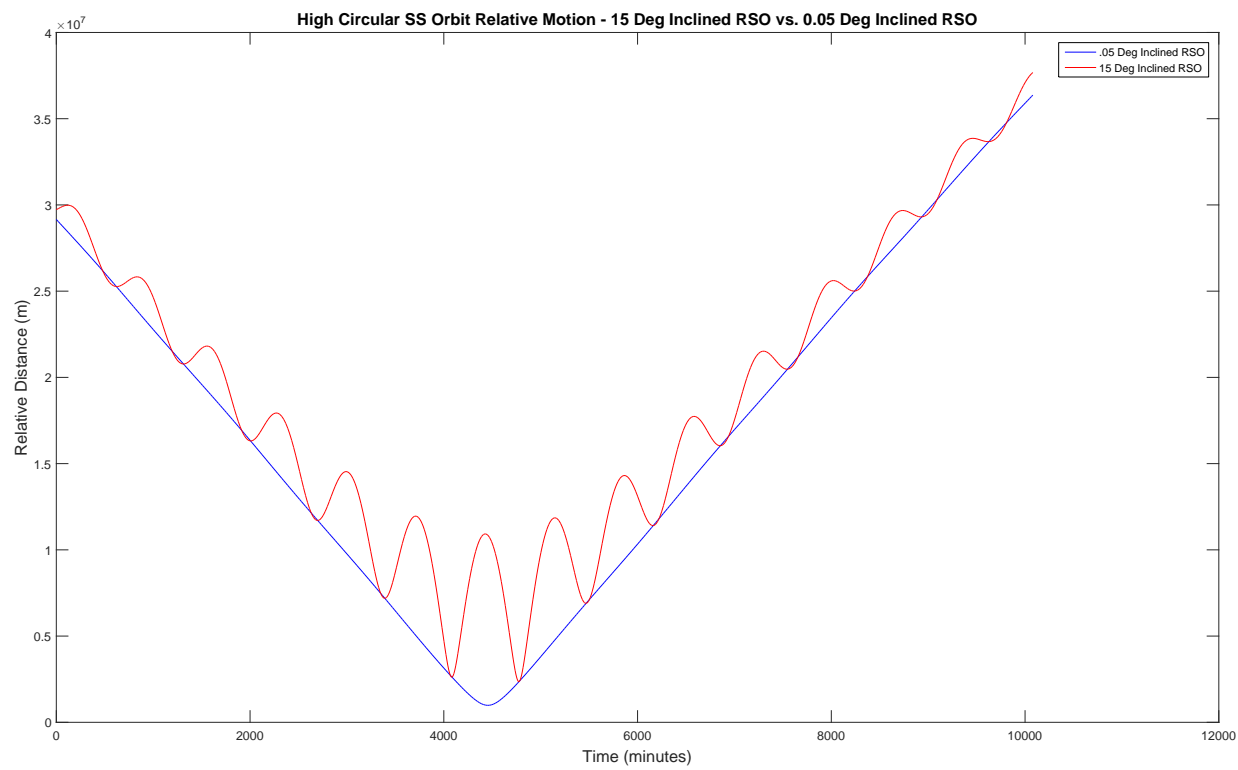

Fig. 13.2: High Circular Space Sensor Relative Motion - RSO Inclined Analysis 


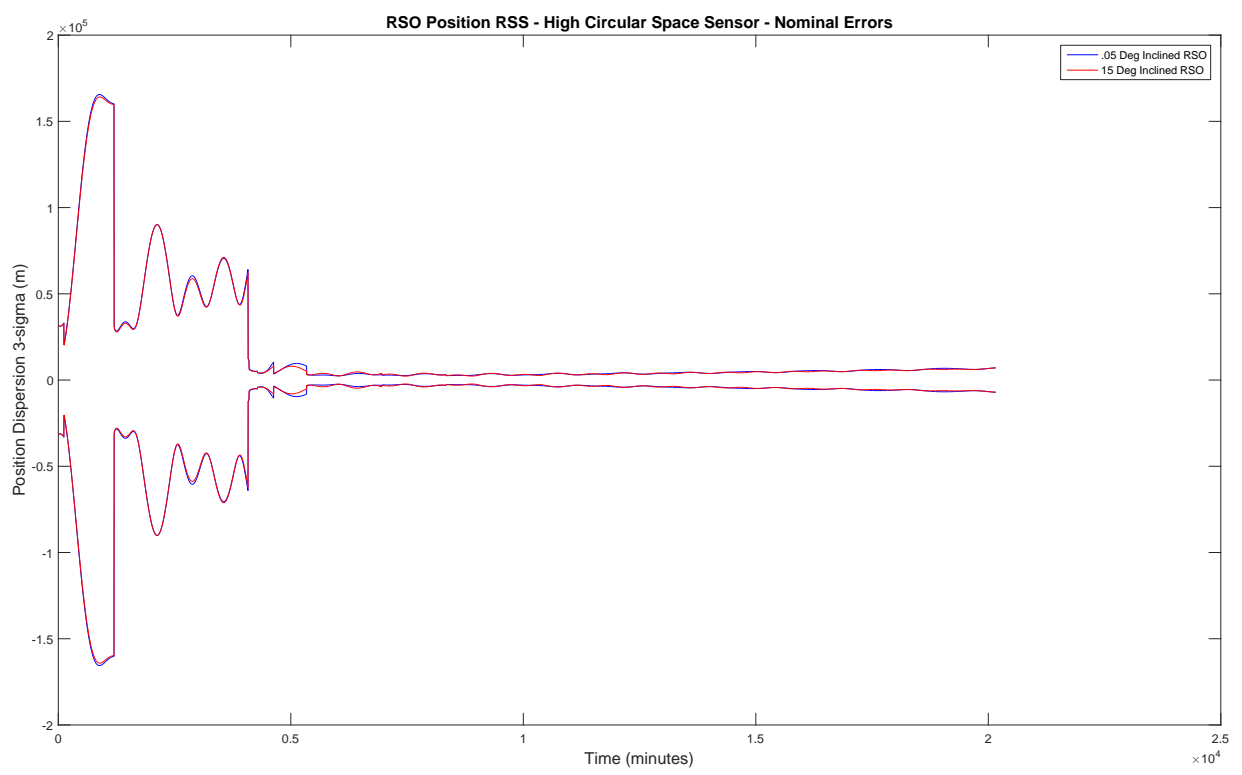

Fig. 13.3: High Circular SS Orbit, Low Initial Errors, RSO Inclination Analysis - RSO 3- $\sigma$ Position Error

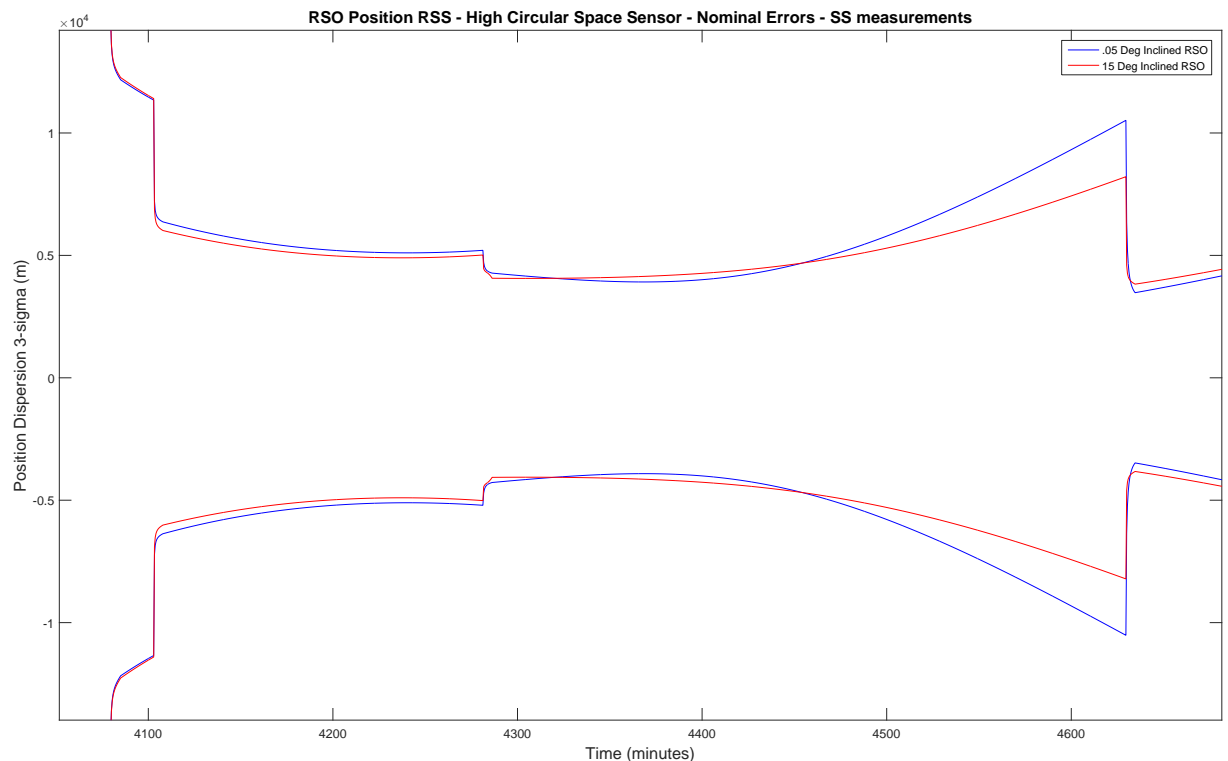

Fig. 13.4: High Circular SS Orbit, Low Errors, RSO Inclination Analysis - RSO 3- $\sigma$ Position Error, During SS Measurement Periods 


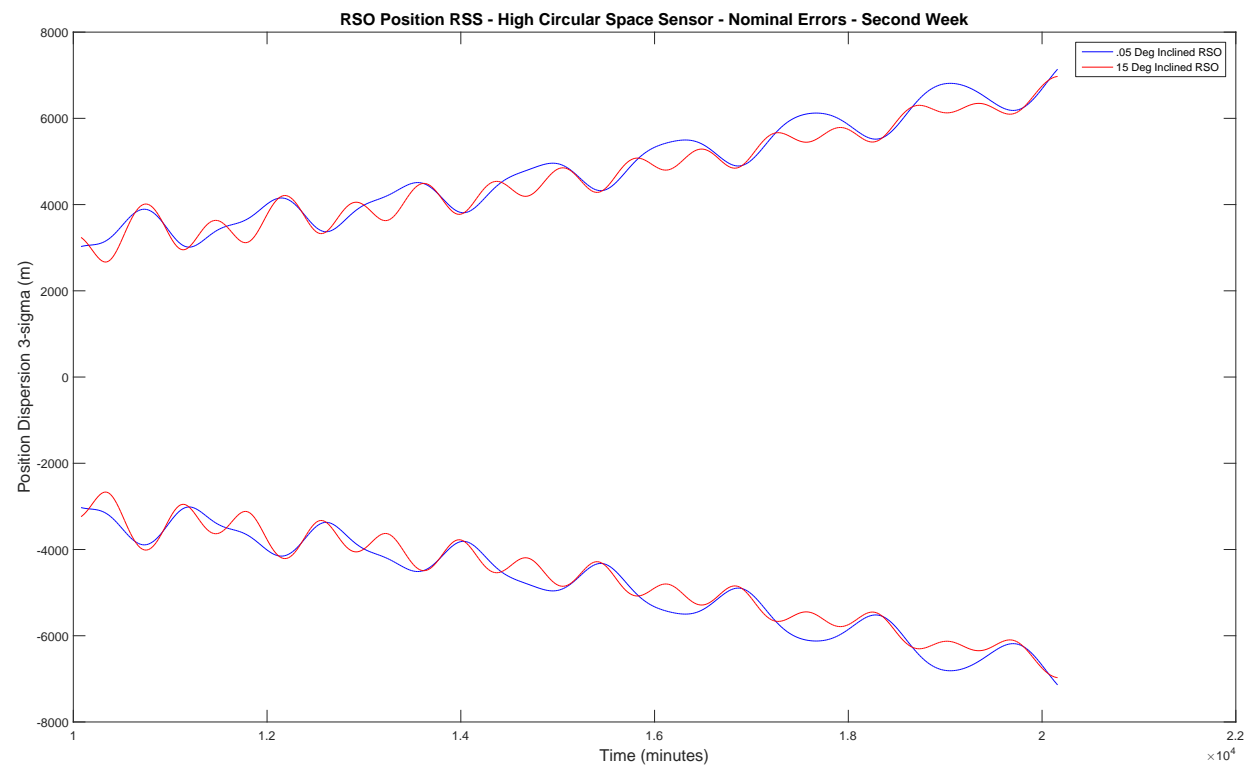

Fig. 13.5: High Circular SS Orbit, Low Errors, RSO Inclination Analysis - RSO 3- $\sigma$ Position Error, During Second Week 
Table 13.3: RSO Inclination Analysis - GS and High Circular SS

\begin{tabular}{|c|c|c|c|}
\hline \multirow{2}{*}{ Point in } & Low Errors & Nominal Errors & High Errors \\
\cline { 2 - 4 } Simulation & \multicolumn{2}{|c|}{$\%$ Change - Position RSS } \\
\hline \hline 1st SS Meas & $-26.0 \%$ & $-5.5 \%$ & $-0.9 \%$ \\
\hline 2nd SS Meas & $+24.9 \%$ & $-4.9 \%$ & $-20.1 \%$ \\
\hline 3rd SS Meas & $-22.1 \%$ & $+10.0 \%$ & $-34.8 \%$ \\
\hline 4th GS Meas & $-41.4 \%$ & $-2.8 \%$ & $-17.3 \%$ \\
\hline 5th GS Meas & $-14.4 \%$ & $+8.8 \%$ & $-4.3 \%$ \\
\hline 6th GS Meas & $-9.1 \%$ & $-2.9 \%$ & $-11.0 \%$ \\
\hline 1 week & $+6.3 \%$ & $+6.95 \%$ & $-19.5 \%$ \\
\hline 2 week & $-2.1 \%$ & $-2.3 \%$ & $-4.2 \%$ \\
\hline
\end{tabular}

\subsection{Elliptical Space Sensor}

Figure 13.6 demonstrates the change in relative motion for the elliptical space sensor orbit. For the elliptical space sensor analysis, it was possible to slightly adjust the space sensor tracking schedule so that the same relative distance between the RSO and space sensor existed at the time of the measurements. The elliptical space sensor measurements still take place between the 3rd and 4th ground station measurement. Table 13.4 presents the results of this analysis. The space sensor measurement results are presented, however they are not directly comparable between the different RSO inclinations due to the measurements taken at different times. Overall, inclining the RSO to 15 degrees made the RSO position accuracy worse at the end of 1 week and 2 weeks, for all error source levels. 


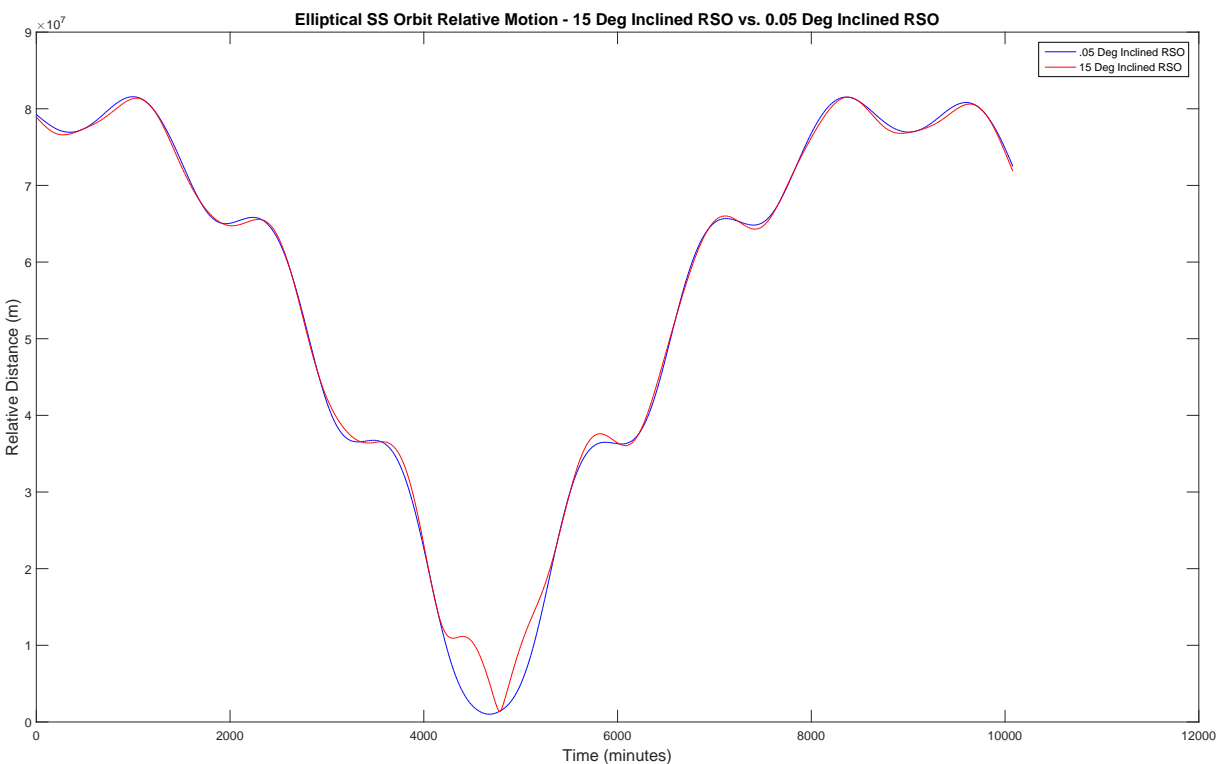

Fig. 13.6: Elliptical Space Sensor Relative Motion - RSO Inclined Analysis 
Table 13.4: RSO Inclination Analysis - GS and Elliptical SS

\begin{tabular}{|c|c|c|c|}
\hline \multirow{2}{*}{ Point in } & Low Errors & Nominal Errors & High Errors \\
\cline { 2 - 4 } Simulation & \multicolumn{2}{|c|}{$\%$ Change - Position RSS } \\
\hline \hline 1st SS Meas & N/A & N/A & N/A \\
\hline 2nd SS Meas & N/A & N/A & N/A \\
\hline 3rd SS Meas & N/A & N/A & N/A \\
\hline 4th GS Meas & $+5.1 \%$ & $+4.4 \%$ & $+9.4 \%$ \\
\hline 5th GS Meas & $+10.7 \%$ & $+21.7 \%$ & $+7.9 \%$ \\
\hline 6th GS Meas & $+3.4 \%$ & $+10.5 \%$ & $+1.2 \%$ \\
\hline 1 week & $+11.4 \%$ & $+18.0 \%$ & $+0.3 \%$ \\
\hline 2 week & $+2.1 \%$ & $+0.05 \%$ & $+1.5 \%$ \\
\hline
\end{tabular}

\subsection{Summary}

The results greatly differ between all the scenarios completed. The ground station only results and the low circular space sensor with ground station results are comparable in that the 15 degree RSO inclination results are slightly better than the 0.05 degree inclination results. However, the results do not vary that greatly whether the RSO is inclined or not, the results improved by no more than 15 percent. This is due to the ground stations and the low circular orbit space sensor being relatively far away from the RSO. Changing the RSO inclination to 15 degrees did not introduce much change in the distance between the RSO and the ground stations or low circular orbit space sensor.

Inclining the RSO to 15 degrees introduced a very different relative geometry between the RSO and the high circular orbit space sensor. The oscillatory motion that resulted can 
be seen in the analysis results. Taking data at one point in time does not give an overall picture of the results, as it is highly dependent upon the oscillatory motion. Overall, the largest improvement seen in the results was approximately $41 \%$.

The elliptical orbit space sensor analysis was handled a bit differently in that the space sensor measurement profile was slightly adjusted to keep the same relative distance between the space sensor and RSO at the time of the measurement. Overall, at the end of 1 week and 2 weeks, the 15 degree RSO inclination results are worse than the 0.05 RSO inclination results, with the largest percent change being approximately $22 \%$. 


\section{CHAPTER 14 \\ RESULTS - SPACE SENSOR INCLINATION ANALYSIS}

\subsection{Results Overview}

This chapter analyzes the effect of inclining the space sensor to a 15 degree inclination from the nominal zero degree inclination. The RSO is set to its nominal inclination. Analysis is completed for each space sensor orbit: low circular, high circular, and elliptical. Results are presented so that a direct comparison can be made between the inclined and zero degree inclination orbit. Changing the space sensor orbit inclination to 15 degrees caused changes in the relative motion of the space sensor and RSO that are very similar to the inclined RSO relative motion.

\subsection{Low Circular Space Sensor}

Setting the low circular space sensor orbit inclination to 15 degrees changed the relative motion range between the RSO and the space sensor slightly, with motion very similar to the inclined RSO relative motion. The nominal space sensor tracking schedule was used for this analysis, however the relative range during the measurement periods will be slightly different than in the zero degree inclined space sensor analysis. RSO RSS position accuracy percent change results are provided after each measurement is taken during the simulation as well as at the 1 week and 2 week points. Table 14.1 presents results for each of the three error levels: low error level, nominal error level, and high error level. Generally, the inclined space sensor results are better than the nominal zero degree inclination orbit, except for a few cases. However, the results do not differ by much. As seen in the RSO inclination analysis, the position of the space sensor in the low circular orbit is relatively far away from the RSO, therefore changing the inclination of the space sensor does not introduce

much of a difference in the results. There is no change in results for the first three ground 
station measurements, as the space sensor has no effect on the results until it takes its first measurement. Therefore, the first three ground station measurements are not presented in the table below.

Table 14.1: SS Inclination Analysis - GS and Low Circular SS

\begin{tabular}{|c|c|c|c|}
\hline \multirow{2}{*}{ Point in } & Low Errors & Nominal Errors & High Errors \\
\cline { 2 - 4 } & \multicolumn{2}{|c|}{$\%$ Change - Position RSS } \\
\hline \hline 1st SS Meas & $-9.07 \%$ & $0 \%$ & $.005 \%$ \\
\hline 2nd SS Meas & $-11.67 \%$ & $4.23 \%$ & $1.08 \%$ \\
\hline 3rd SS Meas & $-5.99 \%$ & $-5.5 \%$ & $-2.55 \%$ \\
\hline 4th GS Meas & $-6.25 \%$ & $-1.91 \%$ & $-0.16 \%$ \\
\hline 5th GS Meas & $-8.45 \%$ & $-1.15 \%$ & $-0.1 \%$ \\
\hline 6th GS Meas & $-5.02 \%$ & $-0.4 \%$ & $-0.02 \%$ \\
\hline 1 week & $-8.66 \%$ & $-0.38 \%$ & $-0.03 \%$ \\
\hline 2 week & $-5.5 \%$ & $-0.28 \%$ & $-0.02 \%$ \\
\hline
\end{tabular}

\subsection{High Circular Space Sensor}

The 15 degree inclined space sensor orbit introduced an oscillatory relative motion with respect to the RSO when compared to the zero degree inclination space sensor, very similar to that seen in the RSO inclination analysis. As a result of this different relative motion, the results of this analysis for the high circular space sensor orbit vary with different error source levels. Figure 14.1 is a plot of the RSO RSS 3-sigma position for the low errors, starting at the space sensor measurements and ending at 2 weeks. The results of the different inclinations cross over each other throughout the simulation. When data is taken at a point 
in time, as presented in Table 14.2, this alone doesn't give a complete picture of the motion. There is no change in results for the first three ground station measurements, as the space sensor has no effect on the results until it takes its first measurement. Therefore, the first three ground station measurements are not presented in Table 14.2.

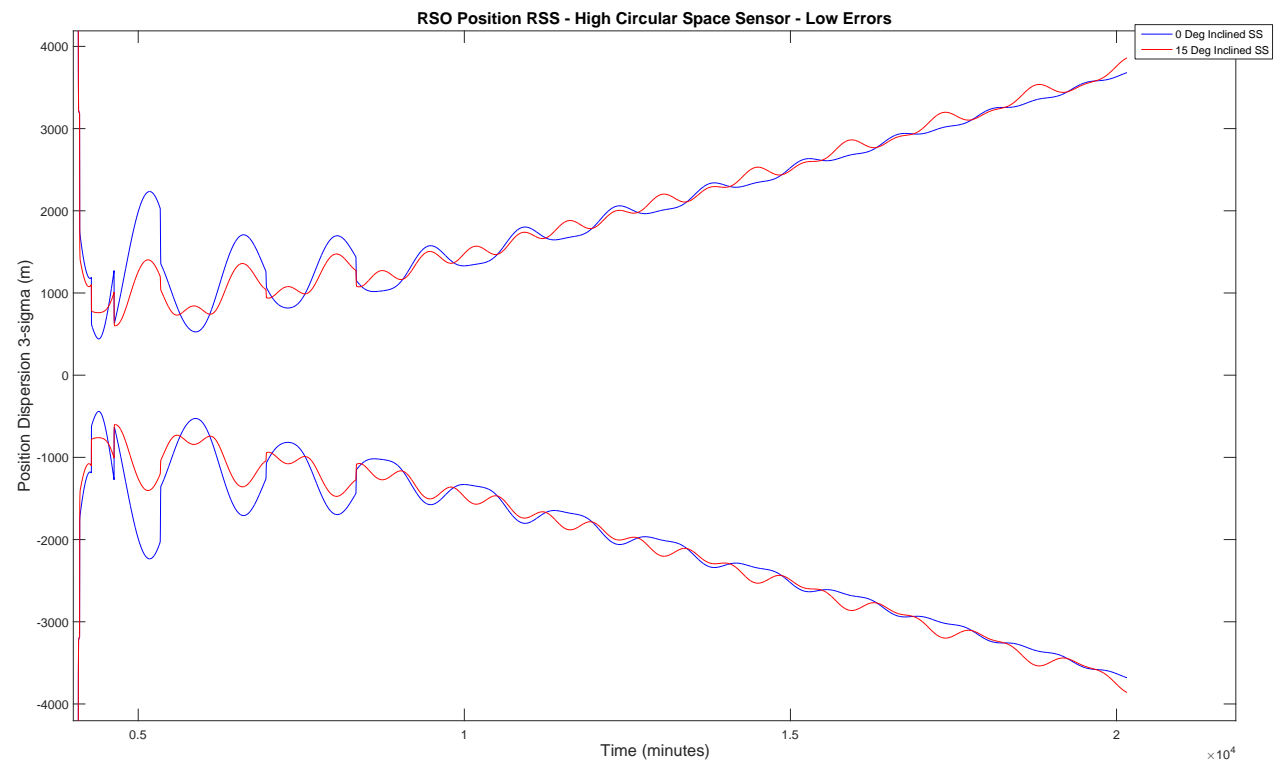

Fig. 14.1: SS Inclination Analysis - RSO 3- $\sigma$ Position Error, Low Initial Errors

Figure 14.2 is a plot of the RSO RSS 3-sigma position for the nominal error level, starting at the space sensor measurements and ending at 2 weeks. The inclined space sensor results are slightly worse than the zero inclination results, as shown in Table 14.2. The high error level results follow the same trend. By examining Figures 14.1 and 14.2 it can be seen that the values in Table 14.2 are very dependent on the time at which the comparison is made. While at times the difference is as much as $+/-27 \%$, Figures 14.2 and 14.2 show that the results are not much different. 


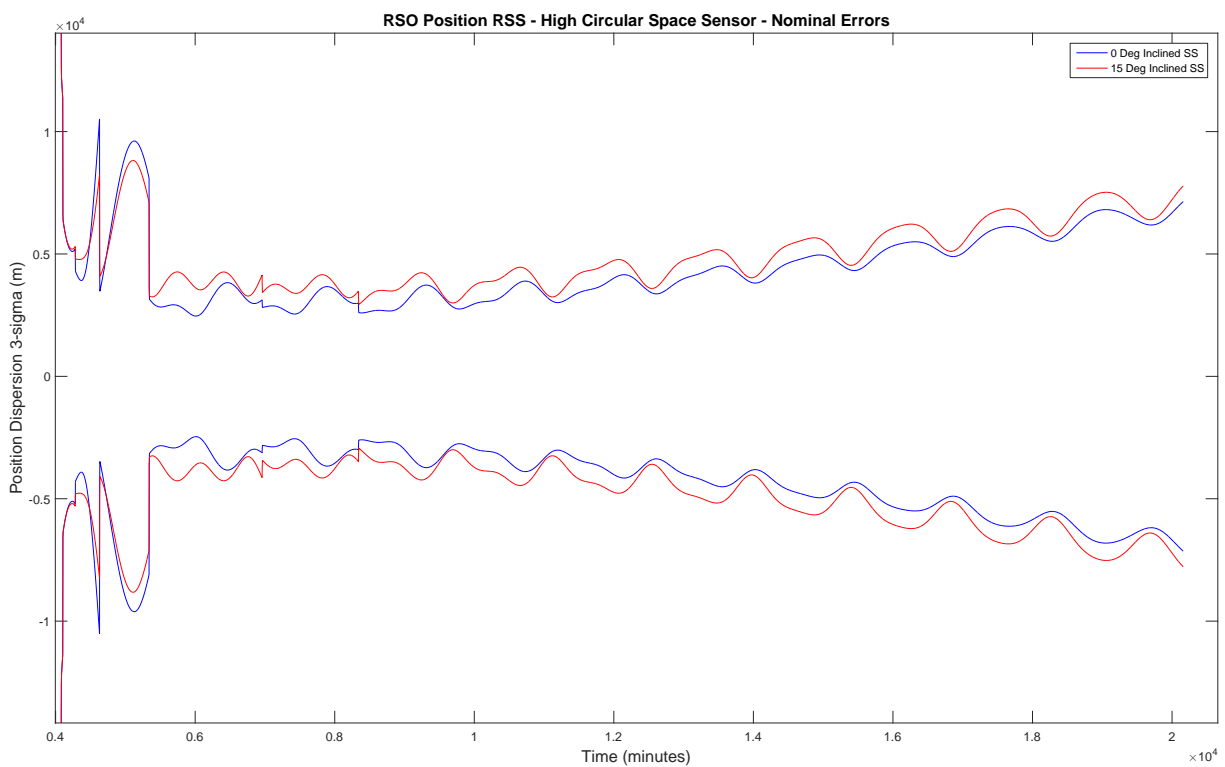

Fig. 14.2: SS Inclination Analysis - RSO 3- $\sigma$ Position Error, Nominal Initial Errors 
Table 14.2: SS Inclination Analysis - GS and High Circular SS

\begin{tabular}{|c|c|c|c|}
\hline \multirow{2}{*}{ Point in } & Low Errors & Nominal Errors & High Errors \\
\cline { 2 - 4 } Simulation & \multicolumn{2}{|c|}{$\%$ Change - Position RSS } \\
\hline \hline 1st SS Meas & $-17.43 \%$ & $-0.58 \%$ & $+0.31 \%$ \\
\hline 2nd SS Meas & $+27.62 \%$ & $+12.16 \%$ & $-5.71 \%$ \\
\hline 3rd SS Meas & $-4.67 \%$ & $+17.71 \%$ & $+4.46 \%$ \\
\hline 4th GS Meas & $-23.53 \%$ & $+4.35 \%$ & $+5.97 \%$ \\
\hline 5th GS Meas & $-11.7 \%$ & $+21.73 \%$ & $+6.65 \%$ \\
\hline 6th GS Meas & $-5.78 \%$ & $+12.82 \%$ & $+2.57 \%$ \\
\hline 1 week & $+15.1 \%$ & $+26.45 \%$ & $+4.11 \%$ \\
\hline 2 week & $+4.87 \%$ & $+8.96 \%$ & $+0.93 \%$ \\
\hline
\end{tabular}

\subsection{Elliptical Space Sensor}

The change in relative motion between the RSO and space sensor, for the inclined space sensor orbit, is very similar to that of the inclined RSO. For this analysis, the same space sensor tracking schedule used in the RSO inclined analysis is used. This keeps relatively the same distance between the RSO and space sensor that existed at the time of the measurements. The elliptical space sensor measurements still take place between the 3rd and 4th ground station measurement. However, the space sensor measurement results are not directly comparable to the zero degree inclination orbit due to the measurements being taken at different times. Generally, the inclined space sensor results are worse than the zero degree inclination orbit, except for one case. There is no change in results for the first three ground station measurements, as the space sensor has no effect on the results 
until it takes its first measurement. Therefore, the first three ground station measurements are not presented in Table 14.3.

Table 14.3: SS Inclination Analysis - GS and Elliptical SS

\begin{tabular}{|c|c|c|c|}
\hline \multirow{2}{*}{ Point in } & Low Errors & Nominal Errors & High Errors \\
\cline { 2 - 4 } Simulation & \multicolumn{2}{|c|}{$\%$ Change - Position RSS } \\
\hline \hline 1st SS Meas & N/A & N/A & N/A \\
\hline 2nd SS Meas & N/A & N/A & N/A \\
\hline 3rd SS Meas & N/A & N/A & N/A \\
\hline 4th GS Meas & $+2.94 \%$ & $+5.44 \%$ & $+11.31 \%$ \\
\hline 5th GS Meas & $+6.37 \%$ & $+18.62 \%$ & $+9.3 \%$ \\
\hline 6th GS Meas & $+3.05 \%$ & $+10.28 \%$ & $+1.95 \%$ \\
\hline 1 week & $+8.9 \%$ & $+7.59 \%$ & $+1.64 \%$ \\
\hline 2 week & $+2.25 \%$ & $-3.38 \%$ & $+1.97 \%$ \\
\hline
\end{tabular}

\subsection{Summary}

The results for the low circular orbit space sensor with ground station measurements do not greatly depend on the space sensor orbit inclination. The low circular orbit space sensor and ground stations are relatively far away from the RSO. Changing the space sensor inclination to 15 degrees did not introduce much change in the distance between the RSO and the low circular orbit space sensor. The largest improvement seen was approximately $12 \%$.

The relative geometry between the RSO and the high circular orbit space sensor 
changed when the space sensor was inclined to 15 degrees, very similar to that of the inclined RSO. The oscillatory motion that resulted can be seen in the analysis results. Taking data at one point in time does not give an overall picture of the results for the low error level. The largest percent change seen in the data presented was approximately $28 \%$.

For the elliptical orbit space sensor analysis the space sensor measurement profile was slightly adjusted to keep the same relative distance between the space sensor and RSO at the time of the measurement. Overall, at the end of 1 week and 2 weeks, the 15 degree space sensor inclination results are worse than the zero inclination results, with the largest percent change being approximately $19 \%$.

Overall, the effect of inclining the space sensor to 15 degrees is dependent on the relative geometry and distance between the space sensor and RSO. In particular, when the inclination causes a very different geometry to exist between the space sensor and the RSO, as with the high circular space sensor, knowledge of the position accuracy over the entire two week period is beneficial, as a snapshot in time does not convey all of the information. 


\section{CHAPTER 15 \\ RESULTS - GPS/USN ANALYSIS}

\subsection{Results Overview}

This chapter first analyzes the effect of changing the space sensor GPS accuracy on RSO orbit determination. Next, the space sensor position and velocity is assumed to be updated through the SSC Universal Space Network, rather than GPS. The SSC USN updates are assumed to occur only once per day.

\subsection{GPS Accuracy}

The geosynchronous satellite GOES-R uses a GPS receiver and antenna as part of the GN\&C suite. GOES-R has an orbit determination accuracy requirement of approximately 150 meter 3-sigma position accuracy. During a worst case test scenario, it was demonstrated to achieve 33.65 meter 3 -sigma position accuracy and $1.98 \mathrm{~cm} / \mathrm{s} 3$-sigma velocity accuracy [35]. To be conservative, for this analysis, the GPS accuracy is raised from the nominal errors of $100 \mathrm{~m}$ in position and $.01 \mathrm{~m} / \mathrm{s}$ in velocity, 1-sigma, to $1 \mathrm{~km}$ in position and 0.1 $\mathrm{m} / \mathrm{s}$ in velocity, 1-sigma. Each space sensor orbit is analyzed, with the space sensor position and velocity updates occurring every 10 minutes. The high level GPS RSO position RSS results are compared to the nominal level GPS RSO position RSS results. Results are

presented for scenarios with 6 ground station measurement periods and only 2 ground station measurement periods. The space sensor measurement profile was kept at nominal. Tables 15.1 through 15.3 present results for each space sensor orbit that includes the percent change in the position RSS when the high GPS position errors are compared to the nominal GPS position errors. The tables include the results for each baseline error source level: low, nominal, and high. Overall, the high GPS error level had hardly any effect on the position RSS results. The largest percent changes were seen in the baseline low error scenarios, but 
the results were still less than a $2 \%$ change.

Table 15.1: GPS Analysis - Low Circular SS Orbit, High GPS Errors

\begin{tabular}{|c|c|c|c|c|}
\hline \multirow{2}{*}{ \# of GS } & & Low Errors & Nominal Errors & High Errors \\
\cline { 3 - 5 } Meas Periods & & \multicolumn{2}{|c|}{$\%$ Change - Position RSS } \\
\hline \hline \multirow{2}{*}{6} & 1 week & $+0.05 \%$ & $+0.002 \%$ & $0 \%$ \\
\cline { 2 - 5 } & 2 week & $+0.02 \%$ & $+0.002 \%$ & $0 \%$ \\
\hline \multirow{2}{*}{2} & 1 week & $+0.21 \%$ & $+0.002 \%$ & $0 \%$ \\
\cline { 2 - 5 } & 2 week & $+0.20 \%$ & $+0.003 \%$ & $0 \%$ \\
\hline
\end{tabular}

Table 15.2: GPS Analysis - High Circular SS Orbit, High GPS Errors

\begin{tabular}{|c|c|c|c|c|}
\hline \multirow{2}{*}{ \# of GS } & & Low Errors & Nominal Errors & High Errors \\
\cline { 3 - 5 } Meas Periods & & \multicolumn{3}{|c|}{$\%$ Change - Position RSS } \\
\hline \hline \multirow{2}{*}{6} & 1 week & $+1.5 \%$ & $+0.15 \%$ & $+0.001 \%$ \\
\cline { 2 - 5 } & 2 week & $+0.5 \%$ & $+0.04 \%$ & $0 \%$ \\
\hline \multirow{2}{*}{2} & 1 week & $+1.7 \%$ & $+0.13 \%$ & $+0.004 \%$ \\
\cline { 2 - 5 } & 2 week & $0.99 \%$ & $+0.08 \%$ & $+0.003 \%$ \\
\hline
\end{tabular}


Table 15.3: GPS Analysis - Elliptical SS Orbit, High GPS Errors

\begin{tabular}{|c|c|c|c|c|}
\hline \multirow{2}{*}{ \# of GS } & & Low Errors & Nominal Errors & High Errors \\
\cline { 3 - 5 } Meas Periods & & \multicolumn{3}{|c|}{ \% Change - Position RSS } \\
\hline \hline \multirow{2}{*}{6} & 1 week & $+1.6 \%$ & $+0.2 \%$ & $+0.001 \%$ \\
\cline { 2 - 5 } & 2 week & $+0.6 \%$ & $+0.07 \%$ & $0 \%$ \\
\hline \multirow{2}{*}{2} & 1 week & $+1.8 \%$ & $+0.1 \%$ & $+0.004 \%$ \\
\cline { 2 - 5 } & 2 week & $+1.2 \%$ & $+0.08 \%$ & $+0.003 \%$ \\
\hline
\end{tabular}

\subsection{SSC USN Accuracy Analysis}

For this analysis, the space sensor position and velocity are updated once per day. The accuracy of this update is based on what is believed could be achieved through use of the SSC USN. Achievable accuracy data could not be found for the SSC USN, however SBV was shown to achieve 15 meter position accuracy when tracked by the USAF Space Ground Link System with 11 tracks per day distributed across eight ground stations [36]. The SSC USN has slightly more globally distributed ground stations [37]. Based on the information found, for this analysis, the best position accuracy achievable was simulated at 20 meters, 1-sigma and a velocity accuracy of $.002 \mathrm{~m} / \mathrm{s}, 1$-sigma. Then the 1-sigma position accuracy was raised to 100 meters, 1000 meters, and 5000 meters, with corresponding velocity accuracies of $.01 \mathrm{~m} / \mathrm{s}, 0.1 \mathrm{~m} / \mathrm{s}$, and $0.5 \mathrm{~m} / \mathrm{s}, 1$-sigma, to simulate less USN ground station tracking per day of the space sensor, which would reduce the cost of using SSC USN. Below, results are presented for scenarios with the nominal 6 ground station measurement periods and only 2 ground station measurement periods. The space sensor measurement profile was kept at nominal. The different levels of accuracy for the SSC USN updates results are compared to what is achievable when nominal level GPS updates are used for the space sensor. Results are only shown for the SSC USN accuracy levels where the RSO position RSS changed by 
over $5 \%$.

For the low circular space sensor orbit, the results for the 20, 100, and 1000 meter accuracy with low errors were less than a $5 \%$ change from the nominal GPS results, therefore they are omitted. For the nominal error source level and high error source level, all of the accuracy levels, including the 5000 meter accuracy cases, changed the results by less than $5 \%$ when compared to the nominal GPS achievable accuracy. The only case in which the errors were more than 5\% are shown in Table 15.4 (low errors, $5000 \mathrm{~m}$ USN accuracy).

Table 15.4: USN Analysis - Low Circular SS Orbit, Low Initial Errors

\begin{tabular}{|c|c|c|}
\hline \multirow{3}{*}{$\begin{array}{c}\text { \# of GS } \\
\text { Meas Periods }\end{array}$} & & USN Accuracy \\
\hline & & $5000 \mathrm{~m}, 0.5 \mathrm{~m} / \mathrm{s}$ \\
\hline & & \% Change - Position RSS \\
\hline \multirow{2}{*}{6} & 1 week & $+0.02 \%$ \\
\hline & 2 week & $+0.9 \%$ \\
\hline \multirow{2}{*}{2} & 1 week & $+30.5 \%$ \\
\hline & 2 week & $+11.7 \%$ \\
\hline
\end{tabular}

For the high circular space sensor orbit, the results for the 20 and 100 meter accuracy for the low errors were less than a 5\% change from the nominal GPS results, therefore they are omitted. For the nominal error source level, the 20 meter and 100 meter results changed less than 5\% and are omitted. Finally, for the high error level all accuracy levels changed the results by less than 5\% when compared to the nominal GPS achievable accuracy. Tables 15.5 and 15.6 show results greater than $5 \%$. 
Table 15.5: USN Analysis - High Circular SS Orbit, Low Initial Errors

\begin{tabular}{|c|c|c|c|}
\hline \multirow{2}{*}{ \# of GS } & & \multicolumn{2}{|c|}{ USN Accuracy } \\
\cline { 3 - 4 } & & $1000 \mathrm{~m}, 0.1 \mathrm{~m} / \mathrm{s}$ & $5000 \mathrm{~m}, 0.5 \mathrm{~m} / \mathrm{s}$ \\
\cline { 3 - 4 } Meas Periods & & \% Change - Position RSS \\
\hline \multirow{2}{*}{6} & 1 week & $+23.9 \%$ & $+70.8 \%$ \\
\cline { 3 - 4 } & 2 week & $+6.08 \%$ & $+18.2 \%$ \\
\hline \multirow{2}{*}{2} & 1 week & $+34.4 \%$ & $+218.3 \%$ \\
\cline { 2 - 4 } & 2 week & $+14.7 \%$ & $+54.9 \%$ \\
\hline
\end{tabular}

Table 15.6: USN Analysis - High Circular SS Orbit, Nominal Initial Errors

\begin{tabular}{|c|c|c|c|}
\hline \multirow{3}{*}{$\begin{array}{c}\text { \# of GS } \\
\text { Meas Periods }\end{array}$} & & \multicolumn{2}{|c|}{ USN Accuracy } \\
\hline & & $1000 \mathrm{~m}, 0.1 \mathrm{~m} / \mathrm{s}$ & $5000 \mathrm{~m}, 0.5 \mathrm{~m} / \mathrm{s}$ \\
\hline & & \multicolumn{2}{|c|}{$\%$ Change - Position RSS } \\
\hline \multirow{2}{*}{6} & 1 week & $+7.06 \%$ & $+40.7 \%$ \\
\hline & 2 week & $+2.28 \%$ & $+12.6 \%$ \\
\hline \multirow{2}{*}{2} & 1 week & $+4.13 \%$ & $+46.8 \%$ \\
\hline & 2 week & $+2.56 \%$ & $+14.2 \%$ \\
\hline
\end{tabular}

For the elliptical space sensor orbit, the results for the 20 and 100 meter accuracy with low errors were less than a 5\% change from the nominal GPS results, therefore they are omitted. For the nominal error source level, the 20 and 100 meter results changed less than 
5\% and are omitted. Finally, for the high error level the 20, 100, and 1000 meter accuracy levels changed the results by less than $5 \%$ when compared to the nominal GPS achievable accuracy. Tables 15.7 through 15.9 show results greater than $5 \%$.

Table 15.7: USN Analysis - Elliptical SS Orbit, Low Initial Errors

\begin{tabular}{|c|c|c|c|}
\hline \multirow{3}{*}{$\begin{array}{c}\text { \# of GS } \\
\text { Meas Periods }\end{array}$} & & \multicolumn{2}{|c|}{ USN Accuracy } \\
\hline & & $1000 \mathrm{~m}, 0.1 \mathrm{~m} / \mathrm{s}$ & $5000 \mathrm{~m}, 0.5 \mathrm{~m} / \mathrm{s}$ \\
\hline & & \multicolumn{2}{|c|}{ \% Change - Position RSS } \\
\hline \multirow{2}{*}{6} & 1 week & $+24.2 \%$ & $+44.2 \%$ \\
\hline & 2 week & $+8.5 \%$ & $+17.1 \%$ \\
\hline \multirow{2}{*}{2} & 1 week & $+39.9 \%$ & $+203.6 \%$ \\
\hline & 2 week & $+24.1 \%$ & $+81.1 \%$ \\
\hline
\end{tabular}

Table 15.8: USN Analysis - Elliptical SS Orbit, Nominal Initial Errors

\begin{tabular}{|c|c|c|c|}
\hline \multirow{2}{*}{ \# of GS } & \multirow{2}{*}{} & \multicolumn{2}{|c|}{ USN Accuracy } \\
\cline { 3 - 4 } Meas Periods & & $1000 \mathrm{~m}, 0.1 \mathrm{~m} / \mathrm{s}$ & $5000 \mathrm{~m}, 0.5 \mathrm{~m} / \mathrm{s}$ \\
\cline { 3 - 4 } & & \multicolumn{2}{|c|}{ \% Change - Position RSS } \\
\hline \multirow{2}{*}{6} & 1 week & $+9.5 \%$ & $+38.9 \%$ \\
\cline { 2 - 4 } & 2 week & $+2.3 \%$ & $+10.3 \%$ \\
\hline \multirow{2}{*}{2} & 1 week & $+5.3 \%$ & $+54.5 \%$ \\
\cline { 2 - 4 } & 2 week & $+3.2 \%$ & $+30.4 \%$ \\
\hline
\end{tabular}


Table 15.9: USN Analysis - Elliptical SS Orbit, High Initial Errors

\begin{tabular}{|c|c|c|}
\hline \multirow{2}{*}{ \# of GS } & & USN Accuracy \\
\cline { 3 - 3 } Meas Periods & & \% Change - Position RSS \\
\cline { 3 - 3 } & & $5000 \mathrm{~m}, 0.5 \mathrm{~m} / \mathrm{s}$ \\
\hline \multirow{2}{*}{6} & 1 week & $+1.8 \%$ \\
\cline { 3 - 3 } & 2 week & $+0.6 \%$ \\
\hline \multirow{2}{*}{2} & 1 week & $+6.7 \%$ \\
\cline { 2 - 3 } & 2 week & $+3.3 \%$ \\
\hline
\end{tabular}

\subsection{Summary}

Varying the GPS accuracy from $100 \mathrm{~m}$ in position and $.01 \mathrm{~m} / \mathrm{s}$ in velocity, 1-sigma, to $1 \mathrm{~km}$ in position and $0.1 \mathrm{~m} / \mathrm{s}$ in velocity, 1-sigma for the space sensor position and velocity updates did not affect the results much. The largest percent changes were seen in the low error levels of the high circular and elliptical space sensor orbit scenarios, however those results only changed by less than $2 \%$.

The SSC USN accuracy analysis produced a greater change in results, especially at the position accuracy levels of 1000 meters and 5000 meters with corresponding velocity accuracy levels of $0.1 \mathrm{~m} / \mathrm{s}$ and $0.5 \mathrm{~m} / \mathrm{s}$. The largest changes were once again seen in the high circular and elliptical space sensor orbit scenarios using the low error source level. To maintain position accuracies near the same level achievable with the nominal GPS accuracy updates, the SSC USN accuracy needs to be $1000 \mathrm{~m}$, 1-sigma, or less in the low circular orbit space sensor, low error level scenarios. For the high circular orbit space sensor, low error and nominal error scenarios, the accuracy needs to be kept at $100 \mathrm{~m}, 1$-sigma, or below. For the elliptical orbit space sensor low error and nominal error scenarios, the accuracy needs 
to be kept at $100 \mathrm{~m}$, 1-sigma, or below. Finally, for the high circular orbit space sensor high error scenario, the accuracy needs to be kept at $1000 \mathrm{~m}$, 1-sigma, or below.

These results clearly show that USN accuracy is relatively unimportant for the baseline high error scenario since in this case the high errors dominate the RSO position accuracy. When the baseline errors are reduced to the low or nominal levels, USN accuracy becomes more important. 


\section{CHAPTER 16 \\ RESULTS - ERROR BUDGET ANALYSIS}

\subsection{Error Budget Analysis Overview}

This chapter analyzes the error budgets for ground station only scenarios and ground station plus space sensor scenarios. It present results for the nominal ground station and space sensor tasking profiles, as well as the effect of reducing the number of ground station measurement periods to two, one for each of the two ground stations. The error budget results for RSO position RSS accuracy, based on the error sources, are broken into eleven groups:

1) RSO initial position and velocity - This set of error sources consists of the initial error placed on the RSO position and RSO velocity.

2) SS initial position and velocity - This set of error sources consists of the initial error placed on the space sensor position and space sensor velocity.

3) RSO area-to-mass ratio errors - This set of error sources consists of the initial error placed on the RSO area-to-mass ratio as well as the random walk noise, $Q_{A_{\text {rso }}}$.

4) SS area-to-mass ratio errors - This set of error sources consists of the initial error placed on the space sensor area-to-mass ratio as well as the random walk noise, $Q_{A_{s s}}$.

5) RSO disturbance accelerations - This consists of the inertial $\mathrm{x}, \mathrm{y}$ and $\mathrm{z}$ components of the RSO disturbance accelerations.

6) SS disturbance accelerations - This consists of the inertial $x, y$ and $z$ components of the space sensor disturbance accelerations.

7) RSO process noise

8) SS process noise

9) GPS/USN errors 
10) Ground station ( $1 \& 2$ ) measurement errors - This set of error sources consists of the ground station measurement biases and noise.

11) SS measurement errors - This consists of the space sensor measurement bias and noise.

This chapter analyzes which of these 11 error groups has the most effect on the RSO position RSS results of a given scenario. Future upgrades and design decisions can be justified by knowing which error groups have the most effect on RSO orbit determination for a given scenario.

\subsection{Ground Station Only}

Figure 16.1 is the error budget breakdown for the nominal ground station measurement only scenario with low error levels. Each of the 11 error groups are represented by a different color/type of line, and the overall position RSS uncertainty is represented by the blue dashed line. At each time step the RSS of the 11 error groups equals the overall position RSS uncertainty. At the start of the simulation the RSO position and velocity errors are dominant but, overtime, as the ground station measurements are incorporated, the ground station errors become dominant. 


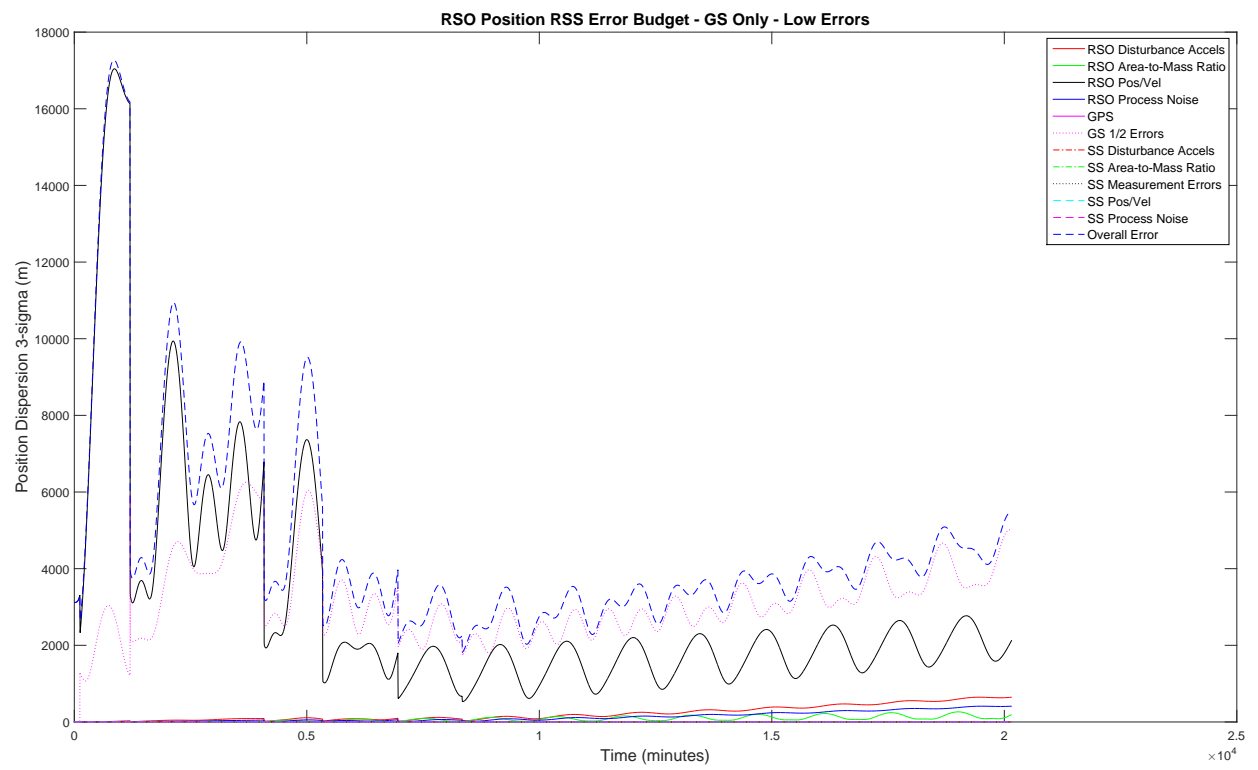

Fig. 16.1: Error Budget Analysis - Ground Station Only, Low Initial Errors

Figure 16.2 shows a close up view of the error budget results from 1 week to 2 weeks. There are no measurements in this stretch of time and the errors are considered to have reached steady error growth values. The errors from the ground station measurements are the most dominant, followed by the RSO initial position and velocity errors, RSO disturbance accelerations, RSO process noise, and finally the RSO area-to-mass ratio. As expected the space sensor sources of error and GPS have no effect on the results since there are no space sensor measurements in this scenario. This information shows that in order to reduce the overall position RSS uncertainty improvements need to be made to the ground station telescopes themselves, in particular the ground station measurement bias and noise. 


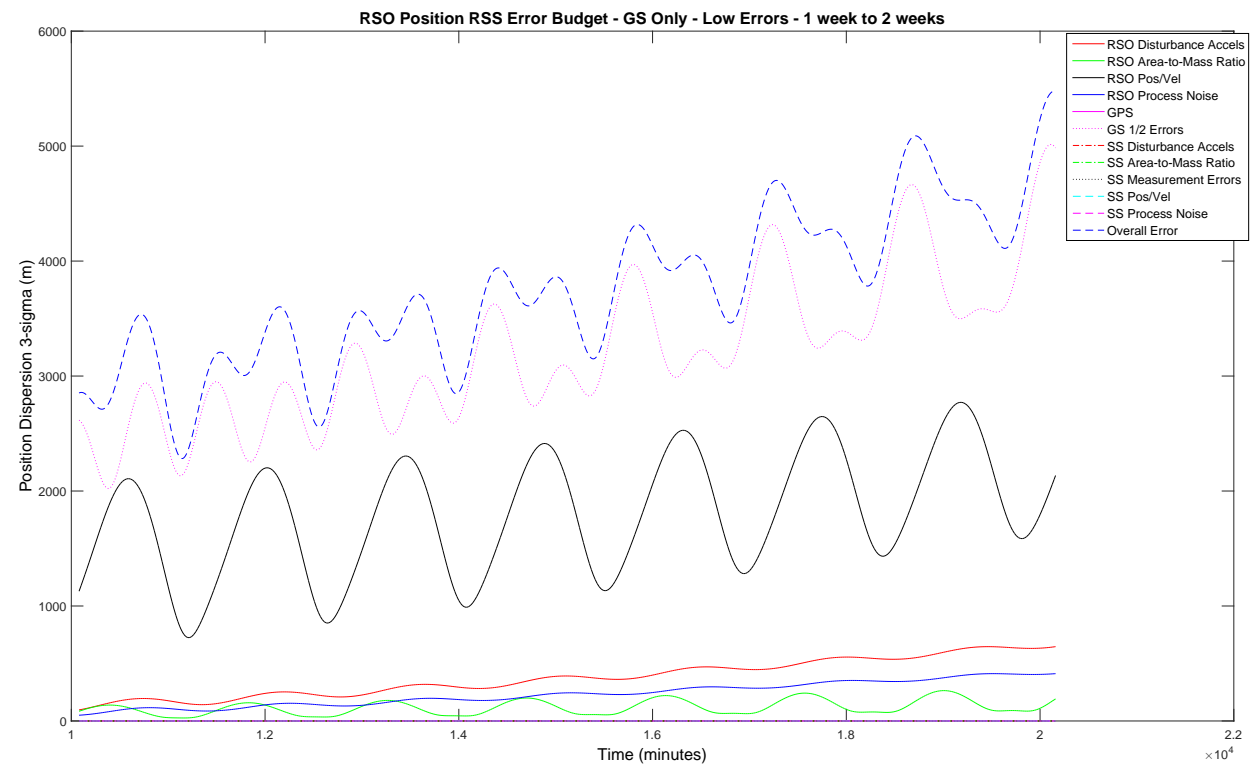

Fig. 16.2: Error Budget Analysis - Ground Station Only, Low Initial Errors, 1 week to 2 weeks

Table 16.1 is a list of the top five error groups for each of the three error source levels for the ground station only nominal tasking scenario. This list shows top error groups once they reach steady error growth, from 1 week to 2 weeks along with their approximate percentage contribution to the overall position RSS error. As a note, it is the RSS of the percentage contributions of all eleven error groups that equals $100 \%$, not the sum.

For the nominal error level, the ground station measurement errors are the most dominant. The RSO process noise becomes more dominant than the RSO initial position and velocity errors by the end of 2 weeks. Finally, the RSO area-to-mass ratio and RSO disturbance accelerations are less dominant.

For the high error level, the RSO process noise becomes the dominant error source. The ground station measurement errors, RSO initial position and velocity errors, RSO areato-mass ratio, and RSO disturbance accelerations are the next dominant error sources in order. 
Table 16.1: Error Budget Analysis - Ground Station Only, Nominal Tasking, 1 week to 2 weeks

\begin{tabular}{|c|c|c|c|}
\hline Error Source Ranking & Low Errors & Nominal Errors & High Errors \\
\hline 1 & $\begin{array}{c}\text { GS Bias and Noise } \\
\qquad \sim 90-95 \%\end{array}$ & $\begin{array}{c}\text { GS Bias and Noise } \\
\qquad 80-85 \%\end{array}$ & $\begin{array}{c}\text { RSO Process Noise } \\
\sim 95 \%\end{array}$ \\
\hline 2 & $\begin{array}{l}\text { RSO Pos/Vel } \\
\quad \sim 35-40 \%\end{array}$ & $\begin{array}{l}\text { RSO Process Noise } \\
\quad \sim 40-50 \%\end{array}$ & $\begin{array}{c}\text { GS Bias and Noise } \\
\qquad \sim 30 \%\end{array}$ \\
\hline 3 & $\begin{array}{l}\text { RSO Dist Accel } \\
\quad \sim 10-15 \%\end{array}$ & $\begin{array}{c}\text { RSO Pos/Vel } \\
\quad \sim 30 \%\end{array}$ & $\begin{array}{l}\text { RSO Pos/Vel } \\
\text { } 10-15 \%\end{array}$ \\
\hline 4 & $\begin{array}{l}\text { RSO Process Noise } \\
\qquad 5-10 \%\end{array}$ & $\begin{array}{c}\text { RSO Area-to-Mass } \\
\sim 25 \%\end{array}$ & $\begin{array}{c}\text { RSO Area-to-Mass } \\
\quad \sim 5-10 \%\end{array}$ \\
\hline 5 & $\begin{array}{c}\text { RSO Area-to-Mass } \\
<5 \%\end{array}$ & $\begin{array}{c}\text { RSO Dist Accel } \\
\sim 10 \%\end{array}$ & $\begin{array}{c}\text { RSO Dist Accel } \\
<5 \%\end{array}$ \\
\hline
\end{tabular}

Figure 16.3 is a plot of the error budget results from 1 week to 2 weeks for the ground station measurement only scenario with only 2 ground station measurement periods and low error levels. It is clearly evident that the dominant sources of error are the RSO initial position and velocity errors and the ground station measurement errors. All other sources of error have a minimal contribution with respect to those two. These are the same top sources of error as in the nominal tasking scenario, however they are much more dominant when there are only 2 ground station measurement periods.

For the nominal error level, the top two dominant errors remain the RSO initial position and velocity errors and the ground station measurement errors. The RSO initial position 
and velocity errors are by far the most dominant. The other listed errors next in order have a minimal contribution in comparison.

For the high error level, the top dominant errors are RSO initial position and velocity errors, the ground station measurement errors, and the RSO process noise. The RSO initial position and velocity errors are by far the most dominant. The RSO process noise took on a more dominant place with this error level. The other listed errors next in order are negligible in comparison.

Table 16.2 summarizes the order of dominant errors groups for each error source level.

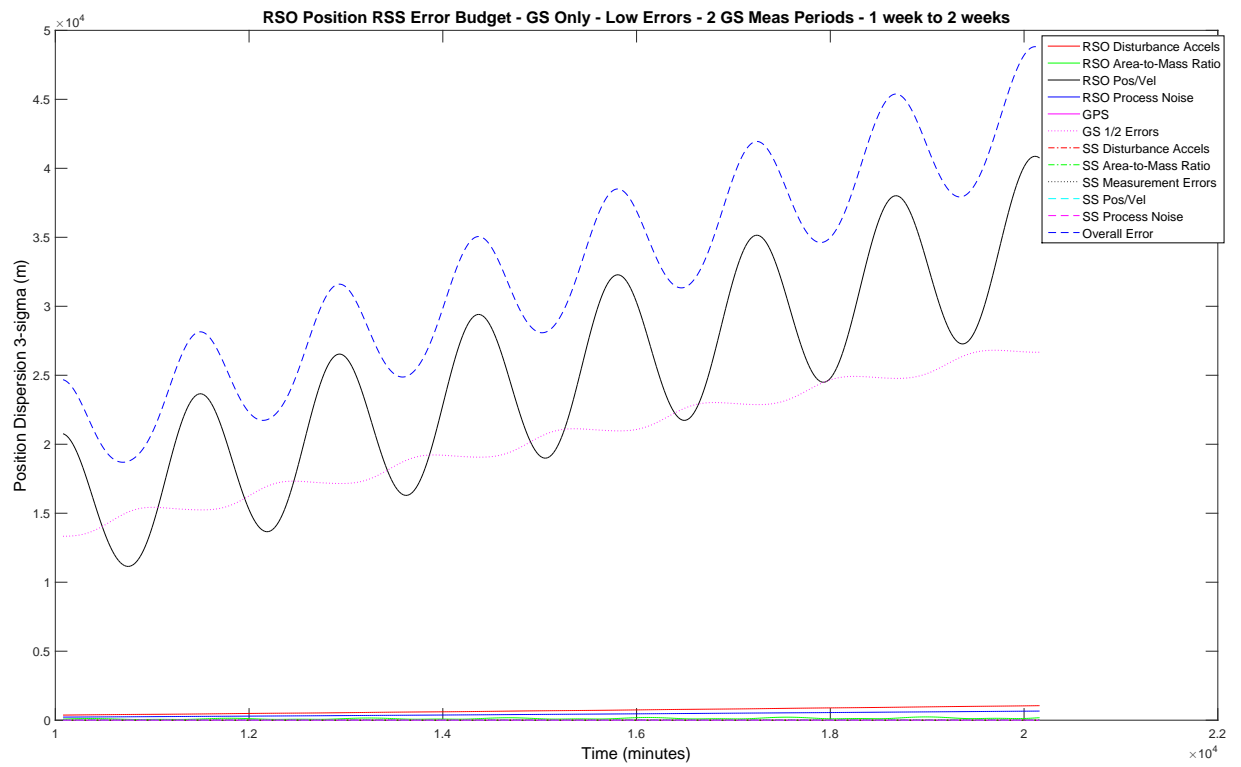

Fig. 16.3: Error Budget Analysis - Ground Station Only, Low Initial Errors, 2 GS Meas Periods, 1 week to 2 weeks 
Table 16.2: Error Budget Analysis - Ground Station Only, 2 GS Tasking, 1 week to 2 weeks

\begin{tabular}{|c|c|c|c|}
\hline Error Source Ranking & Low Errors & Nominal Errors & High Errors \\
\hline 1 & $\begin{array}{l}\text { RSO Pos/Vel } \\
\text { } 70-80 \%\end{array}$ & $\begin{array}{l}\text { RSO Pos/Vel } \\
\quad \sim 90-95 \%\end{array}$ & $\begin{array}{l}\text { RSO Pos/Vel } \\
\text { 〜85-90\% }\end{array}$ \\
\hline 2 & $\begin{array}{l}\text { GS Bias and Noise } \\
\qquad \sim 50-70 \%\end{array}$ & $\begin{array}{c}\text { GS Bias and Noise } \\
\qquad 35-40 \%\end{array}$ & $\begin{array}{c}\text { GS Bias and Noise } \\
\qquad 45-50 \%\end{array}$ \\
\hline 3 & $\begin{array}{c}\text { RSO Dist Accel } \\
<5 \%\end{array}$ & $\begin{array}{c}\text { RSO Process Noise } \\
<5 \%\end{array}$ & $\begin{array}{l}\text { RSO Process Noise } \\
\quad \sim 10-15 \%\end{array}$ \\
\hline 4 & $\begin{array}{c}\text { RSO Process Noise } \\
<5 \%\end{array}$ & $\begin{array}{c}\text { RSO Area-to-Mass } \\
<5 \%\end{array}$ & $\begin{array}{c}\text { RSO Area-to-Mass } \\
<1 \%\end{array}$ \\
\hline 5 & $\begin{array}{c}\text { RSO Area-to-Mass } \\
<5 \%\end{array}$ & $\begin{array}{c}\text { RSO Dist Accel } \\
<5 \%\end{array}$ & $\begin{array}{c}\text { RSO Dist Accel } \\
<1 \%\end{array}$ \\
\hline
\end{tabular}

\subsection{Low Circular Orbit Space Sensor}

Figure 16.4 is the error budget breakdown for low circular space sensor plus ground station measurement scenario for the low error level. At the start of the simulation the RSO position and velocity errors are dominant but, overtime, as the ground station measurements are incorporated, the ground station errors become more important, and as the space sensor begins to take measurements, the space sensor measurement errors become important as well. 


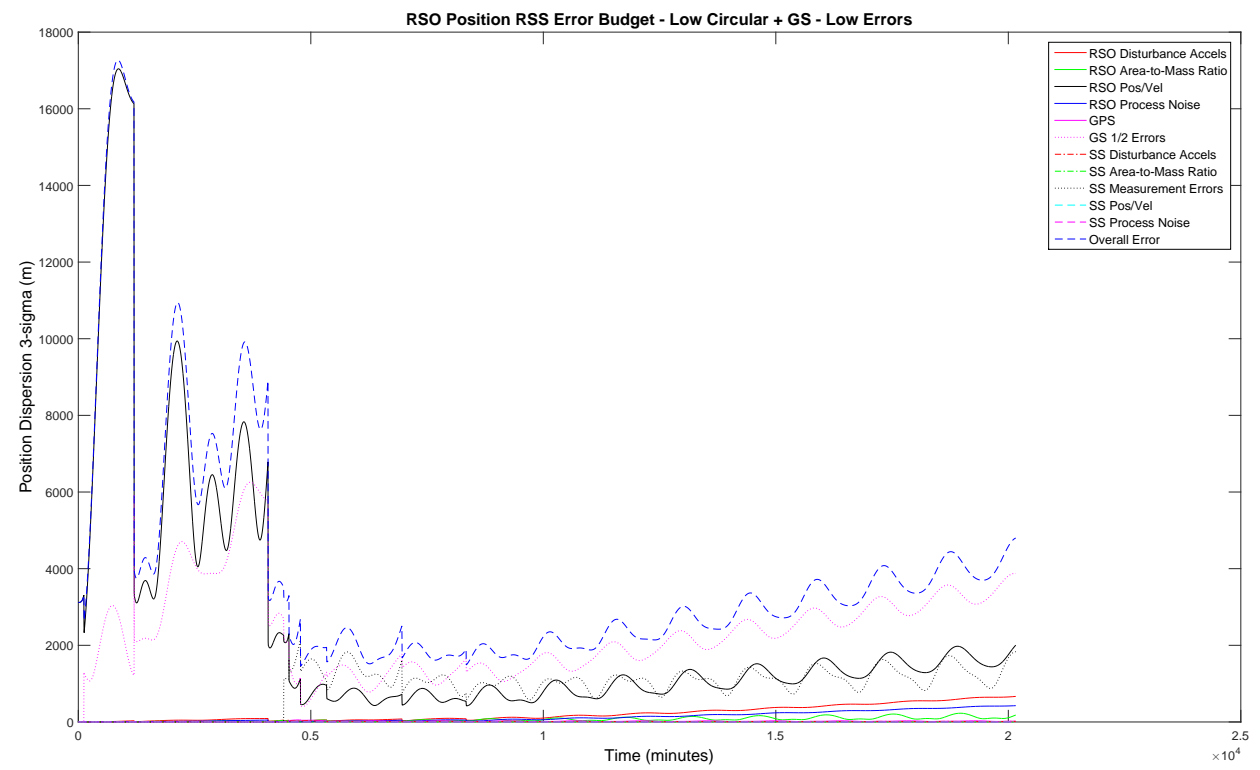

Fig. 16.4: Error Budget Analysis - Low Circular SS + GS, Low Initial Errors

Figure 16.5 is a close up view of the error budget results from 1 week to 2 weeks. There are no measurements in this stretch of time and the errors are considered to have reached steady error growth values. In Figure 16.5, the errors from the ground station measurements are the most dominant, followed by the RSO initial position and velocity errors and space sensor measurement errors. As the two week point approaches, the RSO initial position and velocity errors become more dominant than the space sensor measurement errors. Less dominant, but still prevalent are the RSO disturbance accelerations, RSO process noise, and RSO area-to-mass ratio. This information shows that in order to reduce the overall position RSS uncertainty improvements need to be made to the ground station telescopes themselves, in particular the ground station measurement bias and noise and to the space sensor measurement errors.

For the nominal error level, the errors from the ground station measurements are the most dominant. By the end of 2 weeks, the RSO process noise becomes more dominant than the RSO initial position and velocity errors. Less dominant, but still prevalent is the RSO area-to-mass ratio. To improve the overall position RSS accuracy, focus should be 
placed on reducing the ground station and space sensor measurement errors.

For the high error level, the RSO process noise becomes the most dominant error source. The ground station measurement errors, RSO initial position and velocity errors, space sensor measurement errors, and RSO area-to-mass ratio are the next dominant error sources in order.

Table 16.3 summarizes the order of dominant errors groups for each error source level.

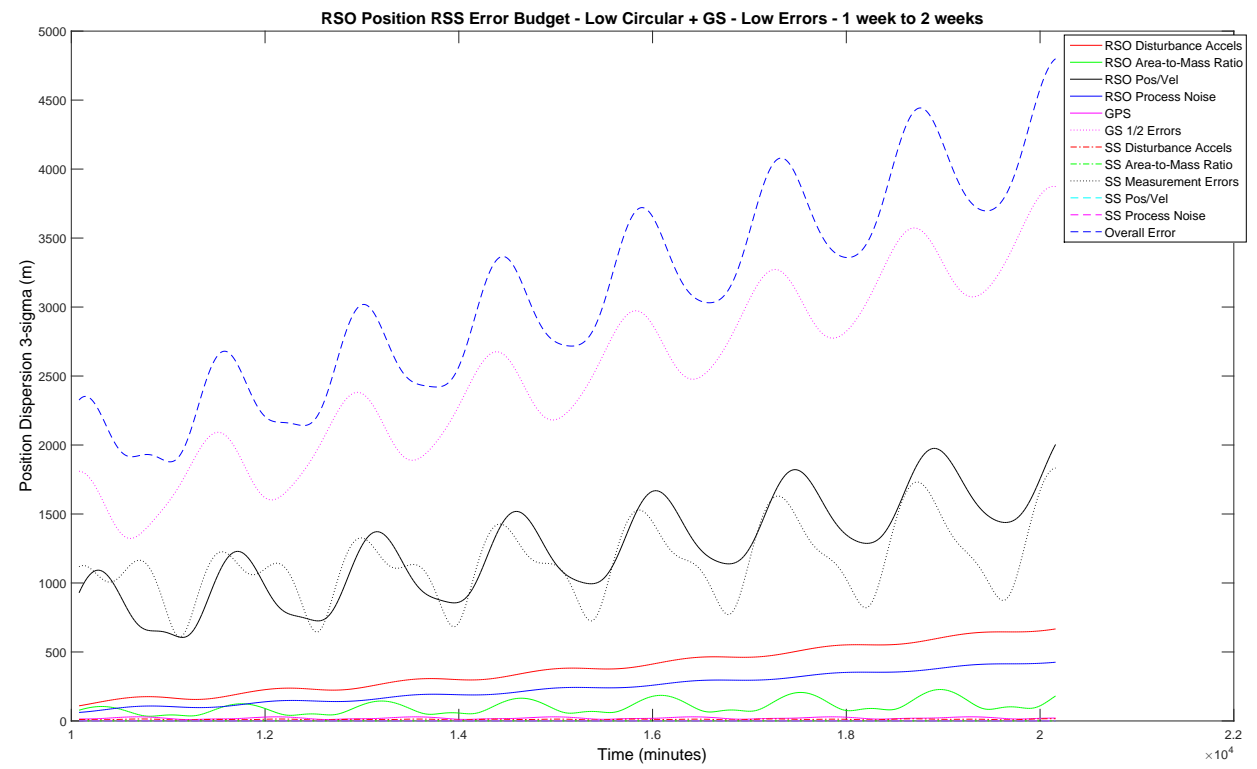

Fig. 16.5: Error Budget Analysis - Low Circular SS + GS, Low Initial Errors, 1 week to 2 weeks 
Table 16.3: Error Budget Analysis - Low Circular SS + GS, Nominal Tasking, 1 week to 2 weeks

\begin{tabular}{|c|c|c|c|}
\hline Error Source Ranking & Low Errors & Nominal Errors & High Errors \\
\hline 1 & $\begin{array}{l}\text { GS Bias and Noise } \\
\qquad \sim 80-90 \%\end{array}$ & $\begin{array}{c}\text { GS Bias and Noise } \\
\quad \sim 65-80 \%\end{array}$ & $\begin{array}{c}\text { RSO Process Noise } \\
\quad \sim 85-90 \%\end{array}$ \\
\hline 2 & $\begin{array}{l}\text { RSO Pos/Vel } \\
\quad \sim 35-40 \%\end{array}$ & $\begin{array}{l}\text { SS Bias and Noise } \\
\quad \sim 40-55 \%\end{array}$ & $\begin{array}{c}\text { GS Bias and Noise } \\
\qquad 30-45 \%\end{array}$ \\
\hline 3 & $\begin{array}{l}\text { SS Bias and Noise } \\
\qquad \sim 30-34 \%\end{array}$ & $\begin{array}{l}\text { RSO Process Noise } \\
\quad \sim 30-50 \%\end{array}$ & $\begin{array}{l}\text { RSO Pos/Vel } \\
\quad \sim 15-25 \%\end{array}$ \\
\hline 4 & $\begin{array}{c}\text { RSO Dist Accel } \\
\sim 15 \%\end{array}$ & $\begin{array}{l}\text { RSO Pos/Vel } \\
\quad \sim 15-30 \%\end{array}$ & $\begin{array}{l}\text { SS Bias and Noise } \\
\qquad \sim 5-10 \%\end{array}$ \\
\hline 5 & $\begin{array}{l}\text { RSO Process Noise } \\
\qquad 10 \%\end{array}$ & $\begin{array}{c}\text { RSO Area-to-Mass } \\
\sim 10 \%\end{array}$ & $\begin{array}{c}\text { RSO Area-to-Mass } \\
\sim 5 \%\end{array}$ \\
\hline
\end{tabular}

Figure 16.6 is a plot of the error budget results from 1 week to 2 weeks for the low circular space sensor plus ground station measurement scenario with only 2 ground station measurement periods and low error levels. It is clearly evident that the dominant source of error is the space sensor measurement noise followed by the RSO initial position and velocity errors and the ground station measurement errors. All other listed sources of error have a minimal contribution with respect to these.

For the nominal error level, the space sensor measurement errors, RSO initial position and velocity errors, and ground station measurement errors are the most dominant. The other listed sources of error are minimal in comparison. 
For the high error level, the RSO initial position and velocity errors, space sensor measurement errors, ground station measurement errors, and RSO process noise are dominant in that order. The other listed source of errors is minimal in comparison.

Table 16.4 summarizes the order of dominant errors groups for each error source level.

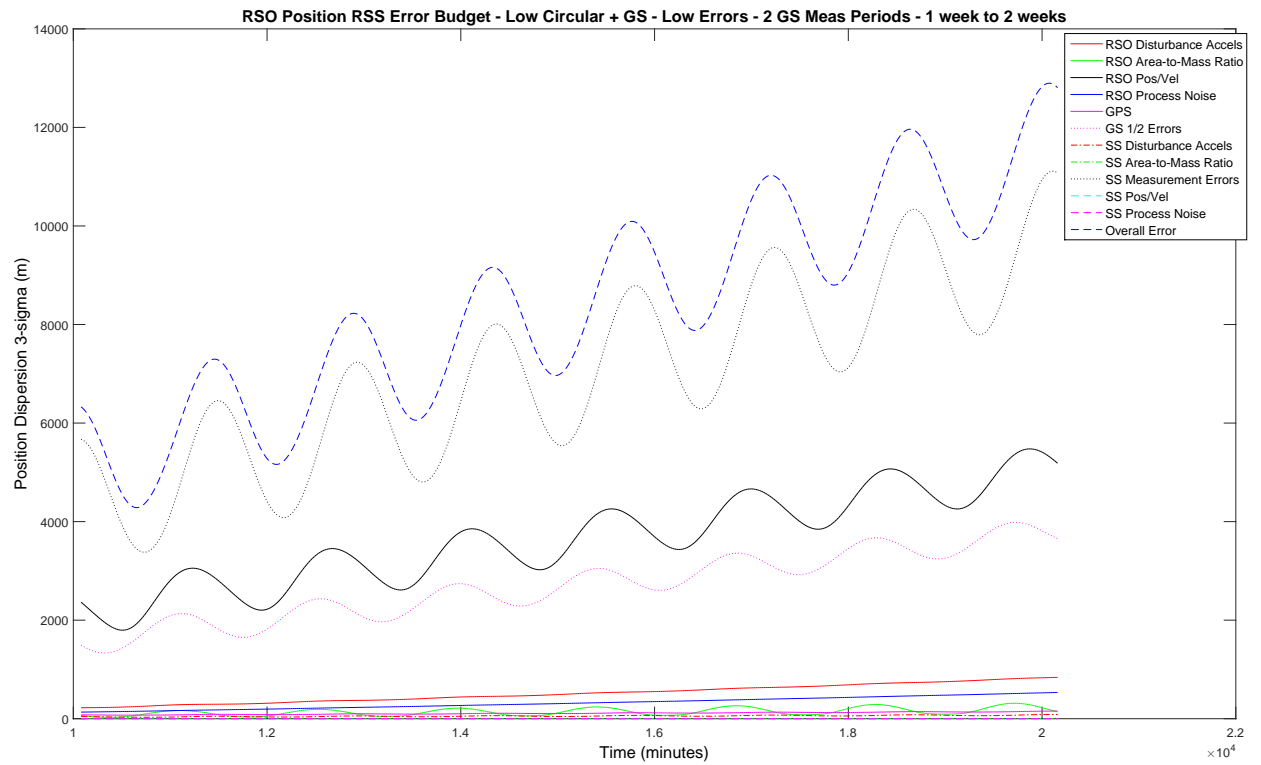

Fig. 16.6: Error Budget Analysis - Low Circular SS + GS, Low Initial Errors, 2 GS Meas Periods, 1 week to 2 weeks 
Table 16.4: Error Budget Analysis - Low Circular SS + GS, 2 GS Tasking, 1 week to 2 weeks

\begin{tabular}{|c|c|c|c|}
\hline Error Source Ranking & Low Errors & Nominal Errors & High Errors \\
\hline 1 & $\begin{array}{l}\text { SS Bias and Noise } \\
\qquad>80-85 \%\end{array}$ & $\begin{array}{l}\text { SS Bias and Noise } \\
\qquad \sim 95 \%\end{array}$ & $\begin{array}{c}\text { RSO Pos/Vel } \\
\quad \sim 75 \%\end{array}$ \\
\hline 2 & $\begin{array}{l}\text { RSO Pos/Vel } \\
\text { 〜40-50\% }\end{array}$ & $\begin{array}{c}\text { RSO Pos/Vel } \\
\quad \sim 25 \%\end{array}$ & $\begin{array}{l}\text { SS Bias and Noise } \\
\quad \sim 50-55 \%\end{array}$ \\
\hline 3 & $\begin{array}{l}\text { GS Bias and Noise } \\
\qquad \sim 30-40 \%\end{array}$ & $\begin{array}{l}\text { GS Bias and Noise } \\
\qquad 20-30 \%\end{array}$ & $\begin{array}{c}\text { GS Bias and Noise } \\
\qquad 40-45 \%\end{array}$ \\
\hline 4 & $\begin{array}{c}\text { RSO Dist Accel } \\
<10 \%\end{array}$ & $\begin{array}{c}\text { RSO Process Noise } \\
\qquad<10 \%\end{array}$ & $\begin{array}{l}\text { RSO Process Noise } \\
\qquad 10-15 \%\end{array}$ \\
\hline 5 & $\begin{array}{c}\text { RSO Process Noise } \\
<10 \%\end{array}$ & $\begin{array}{c}\text { RSO Area-to-Mass } \\
<5 \%\end{array}$ & $\begin{array}{c}\text { RSO Area-to-Mass } \\
<1 \%\end{array}$ \\
\hline
\end{tabular}

\subsection{High Circular Space Sensor Orbit}

Figure 16.7 is a plot of the error budget results from 1 week to 2 weeks for the high circular space sensor plus ground station measurement scenario for low error levels. There are no measurements in this stretch of time and the errors are considered to have reached steady error growth. In Figure 16.7, the errors from the ground station measurements are the most dominant, followed by the RSO initial position and velocity errors. The space sensor measurement errors are less dominant than the RSO disturbance accelerations and RSO process noise by the end of 2 weeks. Less dominant, but still prevalent, is the RSO area- 
to-mass ratio. This information is beneficial in that in order to reduce the overall position RSS uncertainty improvements would need to be made to the ground station telescopes themselves.

For the nominal error level, the errors from the ground station measurements are the most dominant. As the two week point approaches, the RSO process noise becomes more dominant than the space sensor measurement errors and is close to becoming more dominant than the ground station measurement errors. Less dominant, but still prevalent are the RSO area-to-mass ratio and RSO disturbance accelerations.

For the high error level, the most dominant error is the RSO process noise. The ground station measurement errors, space sensor measurement errors, RSO initial position and velocity errors, and RSO area-to-mass ratio are the next dominant in order.

Table 16.5 summarizes the order of dominant errors groups for each error source level.

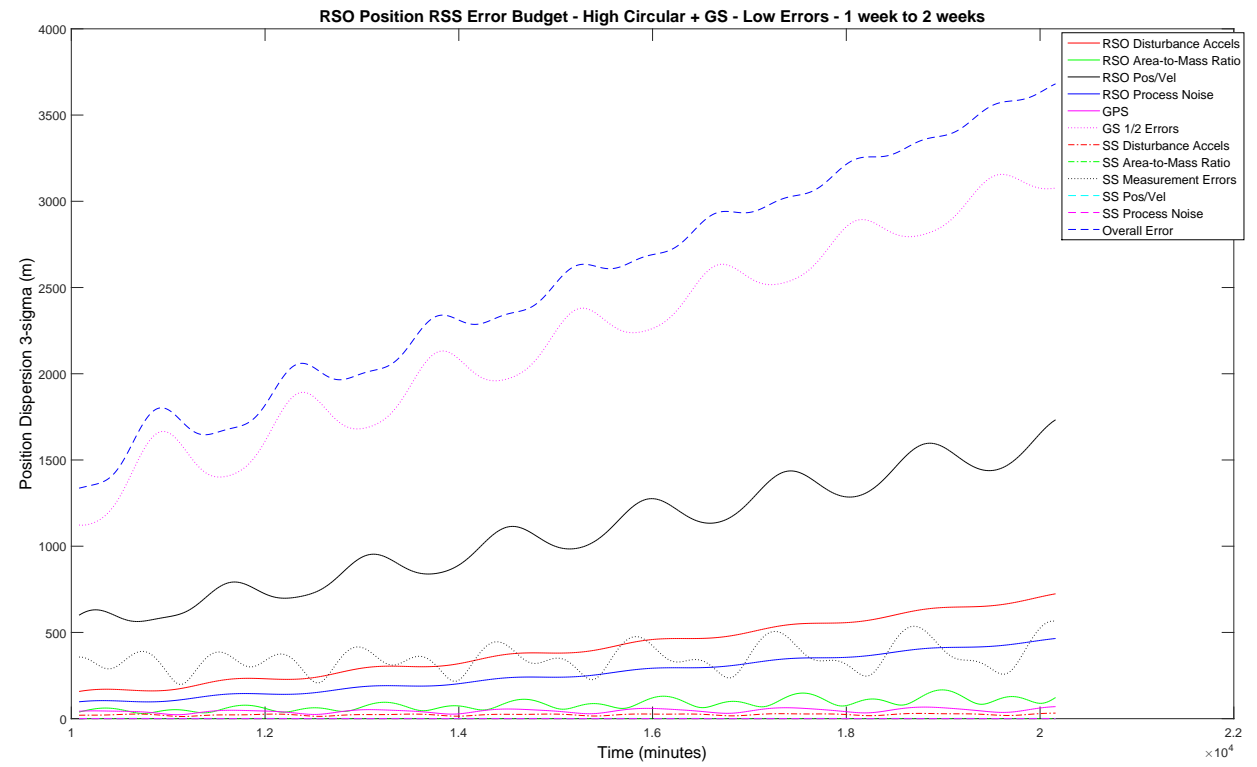

Fig. 16.7: Error Budget Analysis - High Circular SS + GS, Low Initial Errors, 1 week to 2 weeks 
Table 16.5: Error Budget Analysis - High Circular SS + GS, Nominal Tasking, 1 week to 2 weeks

\begin{tabular}{|c|c|c|c|}
\hline Error Source Ranking & Low Errors & Nominal Errors & High Errors \\
\hline 1 & $\begin{array}{l}\text { GS Bias and Noise } \\
\qquad \sim 80-90 \%\end{array}$ & $\begin{array}{c}\text { GS Bias and Noise } \\
\quad \sim 65-70 \%\end{array}$ & $\begin{array}{c}\text { RSO Process Noise } \\
\sim 85 \%\end{array}$ \\
\hline 2 & $\begin{array}{l}\text { RSO Pos/Vel } \\
\quad \sim 40-50 \%\end{array}$ & $\begin{array}{c}\text { RSO Process Noise } \\
\quad \sim 65-70 \%\end{array}$ & $\begin{array}{c}\text { GS Bias and Noise } \\
\qquad 30-35 \%\end{array}$ \\
\hline 3 & $\begin{array}{c}\text { RSO Dist Accel } \\
\quad \sim 20 \%\end{array}$ & $\begin{array}{l}\text { SS Bias and Noise } \\
\qquad 60-70 \%\end{array}$ & $\begin{array}{l}\text { SS Bias and Noise } \\
\qquad 15-30 \%\end{array}$ \\
\hline 4 & $\begin{array}{c}\text { RSO Process Noise } \\
\sim 15 \%\end{array}$ & $\begin{array}{c}\text { RSO Area-to-Mass } \\
\quad \sim 15-25 \%\end{array}$ & $\begin{array}{l}\text { RSO Pos/Vel } \\
\text { } 10-15 \%\end{array}$ \\
\hline 5 & $\begin{array}{l}\text { SS Bias and Noise } \\
\qquad 12 \%\end{array}$ & $\begin{array}{c}\text { RSO Dist Accel } \\
\sim 12 \%\end{array}$ & $\begin{array}{c}\text { RSO Area-to-Mass } \\
\sim 5 \%\end{array}$ \\
\hline
\end{tabular}

Figure 16.8 is a plot of the error budget results from 1 week to 2 weeks for the high circular space sensor plus ground station measurement scenario with only 2 ground station measurement periods and low error levels. The RSO initial position and velocity errors and the ground station measurement errors remain the dominant sources of error, at about equivalent contributions. The space sensor measurement errors, RSO disturbance accelerations, and RSO process noise are the next dominant contributors. All other sources of error have a minimal contribution with respect to these.

For the nominal error level, the dominant source of error is the ground station measurement errors. The space sensor measurement errors are second. The RSO process noise 
begins to become more dominant than the RSO initial position and velocity errors by the end of two weeks. The RSO area-to-mass ratio error is the least dominant. All other sources of error have a minimal contribution with respect to these.

For the high error level, the dominant source of error in this scenario is the space sensor measurement errors. The RSO process noise becomes more dominant than the ground station measurement error by the end of two weeks. The RSO initial position and velocity errors, and RSO area-to-mass ratio are the next dominant sources in order. All other sources of error have a minimal contribution with respect to these.

Table 16.6 summarizes the order of dominant errors groups for each error source level.

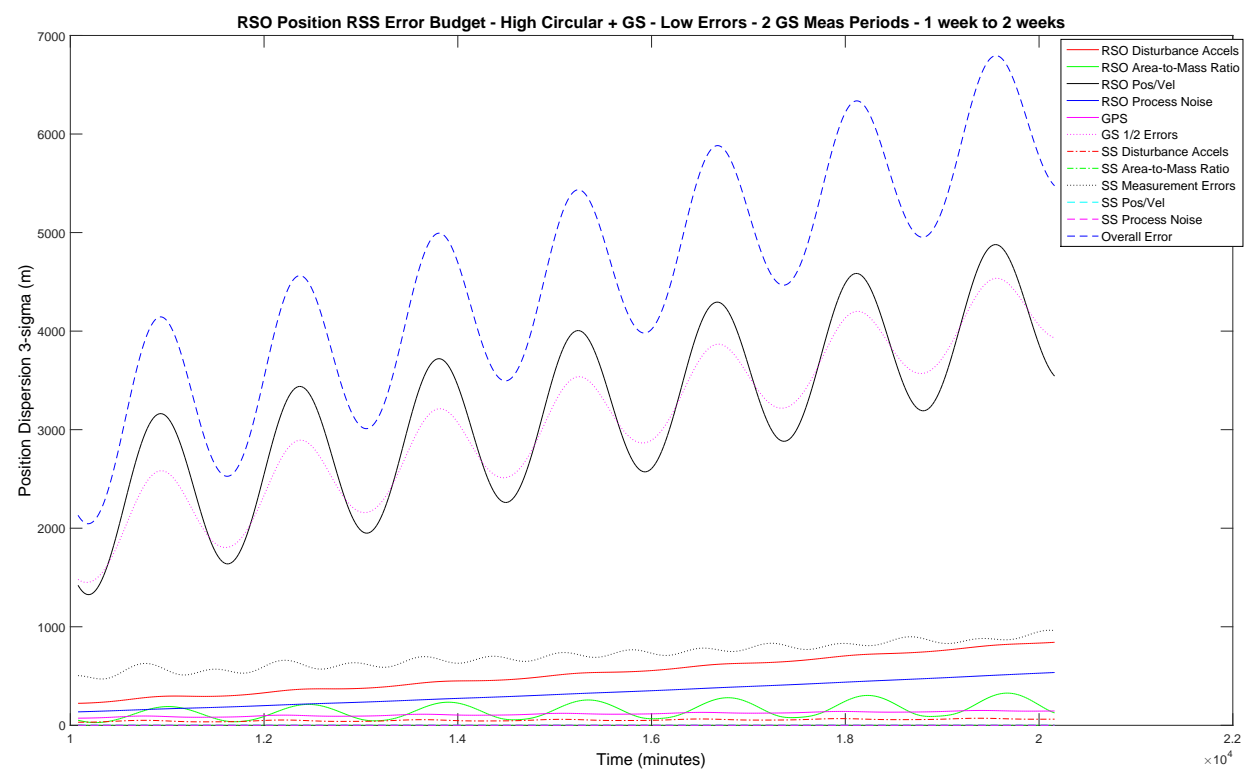

Fig. 16.8: Error Budget Analysis - High Circular SS + GS, Low Initial Errors, 2 GS Meas Periods, 1 week to 2 weeks 
Table 16.6: Error Budget Analysis - High Circular SS + GS, 2 GS Tasking, 1 week to 2 weeks

\begin{tabular}{|c|c|c|c|}
\hline Error Source Ranking & Low Errors & Nominal Errors & High Errors \\
\hline 1 & $\begin{array}{l}\text { RSO Pos/Vel } \\
\text { 〜65-70\% }\end{array}$ & $\begin{array}{c}\text { GS Bias and Noise } \\
\qquad 75-85 \%\end{array}$ & $\begin{array}{l}\text { SS Bias and Noise } \\
\qquad 70-80 \%\end{array}$ \\
\hline 2 & $\begin{array}{c}\text { GS Bias and Noise } \\
\quad \sim 65-75 \%\end{array}$ & $\begin{array}{l}\text { SS Bias and Noise } \\
\quad \sim 35-50 \%\end{array}$ & $\begin{array}{c}\text { RSO Process Noise } \\
\quad \sim 45-55 \%\end{array}$ \\
\hline 3 & $\begin{array}{l}\text { SS Bias and Noise } \\
\quad \sim 15-20 \%\end{array}$ & $\begin{array}{l}\text { RSO Process Noise } \\
\qquad 20-30 \%\end{array}$ & $\begin{array}{c}\text { GS Bias and Noise } \\
\qquad 25-40 \%\end{array}$ \\
\hline 4 & $\begin{array}{l}\text { RSO Dist Accel } \\
\quad \sim 10-15 \%\end{array}$ & $\begin{array}{l}\text { RSO Pos/Vel } \\
\text { } 20-25 \%\end{array}$ & $\begin{array}{l}\text { RSO Pos/Vel } \\
\quad \sim 10-15 \%\end{array}$ \\
\hline 5 & $\begin{array}{c}\text { RSO Process Noise } \\
<10 \%\end{array}$ & $\begin{array}{c}\text { RSO Area-to-Mass } \\
\quad \sim 10-15 \%\end{array}$ & $\begin{array}{c}\text { RSO Area-to-Mass } \\
<5 \%\end{array}$ \\
\hline
\end{tabular}

\subsection{Elliptical Space Sensor Orbit}

Figure 16.9 is a plot of the error budget results from 1 week to 2 weeks for the elliptical space sensor plus ground station measurement scenario for low error levels. There are no measurements in this stretch of time and the errors are considered to have reached steady error growth. The errors from the ground station measurements are the most dominant, followed by the RSO initial position and velocity errors. The space sensor measurement errors become less dominant than the RSO disturbance acceleration and RSO process noise by the end of 2 weeks. 
For the nominal error level, the ground station measurement error remains the most dominant. The RSO process noise becomes more dominant than the space sensor measurement errors by the end of 2 weeks. The RSO area-to-mass ratio and disturbance accelerations are the next dominant error sources in order.

For the high error level, the RSO process noise is now the most dominant. The space sensor and ground station measurement errors are the next dominant error sources in order. The least dominant error groups are the RSO initial position and velocity errors and the RSO area-to-mass ratio.

Table 16.7 summarizes the order of dominant errors groups for each error source level.

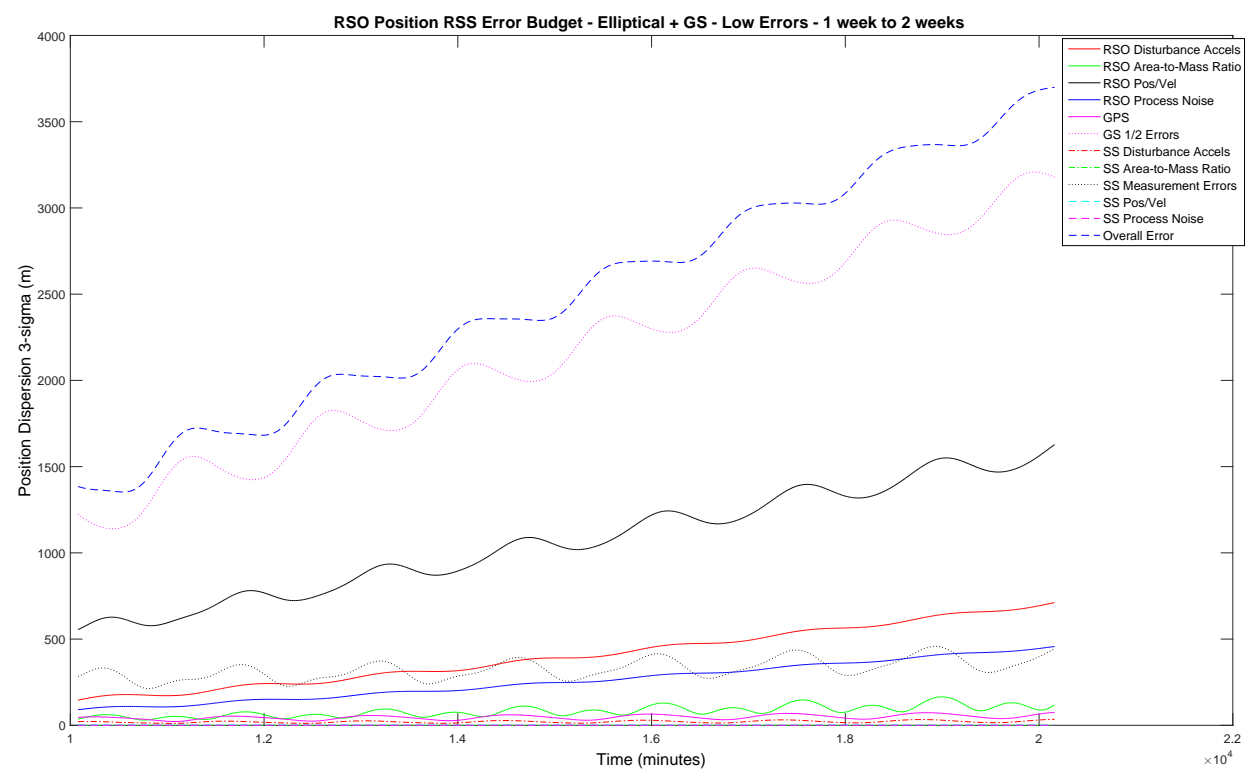

Fig. 16.9: Error Budget Analysis - Elliptical SS + GS, Low Initial Errors, 1 week to 2 weeks 
Table 16.7: Error Budget Analysis - Elliptical SS + GS, Nominal Tasking, 1 week to 2 weeks

\begin{tabular}{|c|c|c|c|}
\hline Error Source Ranking & Low Errors & Nominal Errors & High Errors \\
\hline 1 & $\begin{array}{c}\text { GS Bias }+ \text { Noise } \\
\sim 85-90 \%\end{array}$ & $\begin{array}{c}\text { GS Bias }+ \text { Noise } \\
\sim 65-70 \%\end{array}$ & $\begin{array}{c}\text { RSO Process Noise } \\
\quad \sim 90-95 \%\end{array}$ \\
\hline 2 & $\begin{array}{l}\text { RSO Pos/Vel } \\
\quad \sim 45-50 \%\end{array}$ & $\begin{array}{l}\text { RSO Process Noise } \\
\quad \sim 50-65 \%\end{array}$ & $\begin{array}{c}\text { SS Bias }+ \text { Noise } \\
\quad \sim 20-40 \%\end{array}$ \\
\hline 3 & $\begin{array}{c}\text { RSO Dist Accel } \\
\quad \sim 20 \%\end{array}$ & $\begin{array}{c}\text { SS Bias + Noise } \\
\quad \sim 30-40 \%\end{array}$ & $\begin{array}{c}\text { GS Bias }+ \text { Noise } \\
\quad \sim 25-30 \%\end{array}$ \\
\hline 4 & $\begin{array}{c}\text { RSO Process Noise } \\
\sim 15 \%\end{array}$ & $\begin{array}{c}\text { RSO Area-to-Mass } \\
\quad \sim 25-35 \%\end{array}$ & $\begin{array}{l}\text { RSO Pos/Vel } \\
\quad \sim 5-15 \%\end{array}$ \\
\hline 5 & $\begin{array}{c}\mathrm{SS} \text { Bias }+ \text { Noise } \\
\sim 10-15 \%\end{array}$ & $\begin{array}{c}\text { RSO Dist Accel } \\
\sim 15 \%\end{array}$ & $\begin{array}{c}\text { RSO Area-to-Mass } \\
<5 \%\end{array}$ \\
\hline
\end{tabular}

Figure 16.10 is a plot of the error budget results from 1 week to 2 weeks for the elliptical space sensor plus ground station measurement scenario with only 2 ground station measurement periods and low error levels. The RSO initial position and velocity errors and the ground station measurement errors remain the dominant sources of error, at about equivalent contributions. The space sensor measurement errors, RSO disturbance accelerations, RSO process noise, RSO area-to-mass ratio are the next dominant error sources in order. All other sources of error have a minimal contribution with respect to these.

For the nominal error level, the ground station and space sensor measurement errors are the most dominant sources of error. The RSO initial position and velocity errors, RSO 
process noise, and RSO area-to-mass ratio are the next dominant error sources in order.

For the high error level, the space sensor measurement errors are dominant. The RSO process noise becomes more dominant than the ground station bias and noise by the end of two weeks. The RSO initial position and velocity errors and RSO area-to-mass ratio are the next dominant error sources in order.

Table 16.8 summarizes the order of dominant errors groups for each error source level.

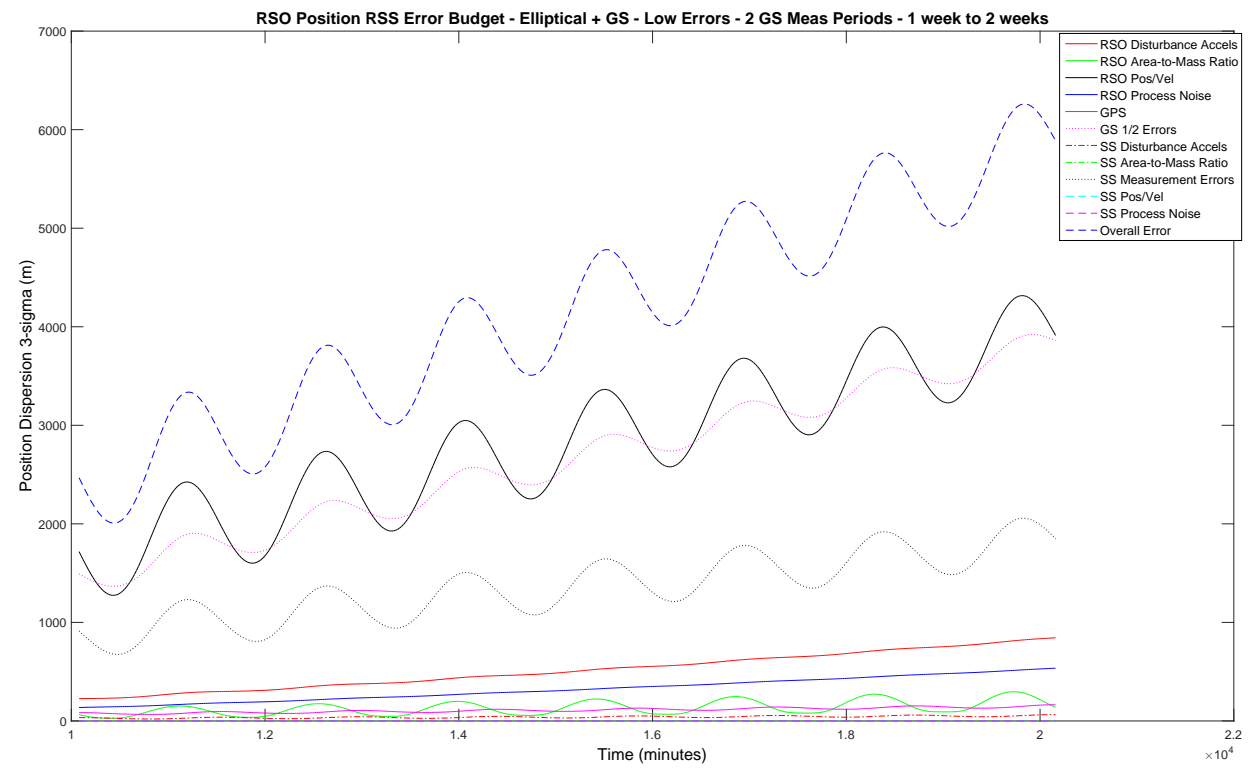

Fig. 16.10: Error Budget Analysis - Elliptical SS + GS, Low Initial Errors, 2 GS Meas Periods, 1 week to 2 weeks 
Table 16.8: Error Budget Analysis - Elliptical SS + GS, 2 GS Tasking, 1 week to 2 weeks

\begin{tabular}{|c|c|c|c|}
\hline Error Source Ranking & Low Errors & Nominal Errors & High Errors \\
\hline 1 & $\begin{array}{l}\text { RSO Pos/Vel } \\
\quad \sim 65-70 \%\end{array}$ & $\begin{array}{c}\text { GS Bias }+ \text { Noise } \\
\sim 80-85 \%\end{array}$ & $\begin{array}{c}\text { SS Bias }+ \text { Noise } \\
\quad \sim 75-85 \%\end{array}$ \\
\hline 2 & $\begin{array}{c}\text { GS Bias }+ \text { Noise } \\
\sim 65 \%\end{array}$ & $\begin{array}{c}\text { SS Bias }+ \text { Noise } \\
\sim 40-45 \%\end{array}$ & $\begin{array}{l}\text { RSO Process Noise } \\
\qquad 45-50 \%\end{array}$ \\
\hline 3 & $\begin{array}{c}\text { SS Bias + Noise } \\
\quad \sim 30-35 \%\end{array}$ & $\begin{array}{l}\text { RSO Pos/Vel } \\
\quad \sim 25-30 \%\end{array}$ & $\begin{array}{c}\text { GS Bias + Noise } \\
\sim 30-40 \%\end{array}$ \\
\hline 4 & $\begin{array}{l}\text { RSO Dist Accel } \\
\quad \sim 15 \%\end{array}$ & $\begin{array}{l}\text { RSO Process Noise } \\
\qquad 20-25 \%\end{array}$ & $\begin{array}{l}\text { RSO Pos/Vel } \\
\quad \sim 5-10 \%\end{array}$ \\
\hline 5 & $\begin{array}{c}\text { RSO Process Noise } \\
\sim 10 \%\end{array}$ & $\begin{array}{c}\text { RSO Area-to-Mass } \\
\quad \sim 5-15 \%\end{array}$ & $\begin{array}{c}\text { RSO Area-to-Mass } \\
<5 \%\end{array}$ \\
\hline
\end{tabular}

\subsection{Summary}

A consistent dominant error group throughout all of the scenarios is the ground station measurement errors. In order to reduce the overall RSS position error, the ground station measurement bias and/or noise needs to be reduced.

For the ground station measurement only, 2 ground station measurement periods scenarios, the RSO initial position and velocity error is the most dominant for each error level, followed by the ground station measurement errors. In comparison, when the measurement tasking scenario was nominal, the ground station measurement errors were a dominant source of error. When the measurement periods are reduced to two, the ground stations 
can no longer reduce the RSO initial position and velocity error to a low value, therefore the initial errors are the most dominant.

It's interesting to note that for the ground station only and ground station plus space sensor scenario, with the nominal tasking, the high error level dominant error group is the RSO process noise. This process noise was meant to model unknown dynamics effects, such as the SRP on a high area-to-mass ratio object where the dynamics of the object are mismodeled. Better knowledge and modeling of these objects would need incorporated to reduce the RSO process noise.

It's also interesting to note that the elliptical space sensor results almost exactly mirror the high circular space sensor results. This seems to fall in line with the results of the previous chapters, where the numerical results from both the elliptical and high circular space sensor orbits are similar.

GPS has virtually no effect on the error budget results. This confirms the results seen in the GPS results section, when changing the GPS accuracy had very little effect on the position RSS values. The space sensor area-to-mass ratio errors also had virtually no effect on the results as well. This is an expected result as the space sensor vehicle was set to a relatively well-known area to mass ratio for all scenarios. It's interesting to note that the space sensor initial position and velocity errors and the disturbance accelerations had no effect on the results. These were in most cases at least an order of magnitude or two less than the RSO values for the similar quantities, therefore their effect is minimal in comparison to the RSO effect of these error groups. 


\section{CHAPTER 17 \\ CONCLUSIONS}

\subsection{Dissertation Thesis Statement}

Through linear covariance theory the increased orbital accuracy of objects in the GEO catalog, achievable when the AFSPC SSN is augmented with space-based angles-only measurements from a sensor in a near-GEO orbit, can be quantified.

\subsection{Summary of Results}

The LinCov analysis tool developed and implemented in this dissertation demonstrates that the increased orbital accuracy of objects in the GEO catalog, encompassing both AFSPC SSN sensor measurements and space-based angles-only measurements from a space sensor, can be quantified using linear covariance theory. First, a truth model was developed to simulate as closely as possible, the complete dynamics and environment of the RSO, space sensor, and ground stations. Within the truth model, SS and ground station measurements

of the RSO were generated. A developed EKF processed these measurements to determine the orbital accuracy of the RSO. The EKF results were compared to Monte Carlo results and verified that the EKF performs within its intended application. The truth model and EKF were then linearized around a reference trajectory to develop the LinCov analysis tool. This LinCov analysis tool was used to determine the orbital accuracy of an RSO, while incorporating both AFSPC SSN ground station and space sensor angles-only measurements.

The baseline results created with the LinCov analysis tool clearly demonstrate that the orbital accuracy of the RSO increased when space-based angles-only measurements from a space sensor were used to augment the two simulated AFSPC SSN ground station measurements. Increased orbital accuracy occurred when space sensor measurements were taken from all three space sensor orbits: low circular, high circular, and elliptical, as well 
as at all three error source levels: low, nominal, and high. Reducing the number of ground station measurement periods, from the nominal value of 6 down to 2 resulted in worse RSO position RSS accuracy. Extending the space sensor measurement periods from 5 minutes to 20 minutes increases the orbital accuracy in all scenarios.

Ground station only and ground station plus low circular space sensor orbit results for a 15 degree inclined RSO changed less than 15\%. Both the ground stations and low circular space sensor orbit are relatively far away from the RSO; therefore changing the RSO inclination did not have much of an effect on the results. Similarly, when the space sensor is inclined to 15 degrees, the ground station plus low circular space sensor orbit results changed by at most $12 \%$. These results confirm that inclination change does not have much of an effect on ground station only or ground station plus low circular space sensor orbit results. The relative geometry between the high circular space sensor and RSO changed dramatically when either the RSO or the space sensor was inclined to 15 degrees. This resulted in widely varying results. A comprehensive assessment overall all conditions is required to determine the change in accuracy as opposed to just examining data at certain points in time.

GPS position and velocity accuracy does not have an effect on the position accuracy of the RSO for the GPS high initial error parameters of $1 \mathrm{~km}$ 1-sigma position error and $0.1 \mathrm{~m} / \mathrm{s}$ 1-sigma velocity error, the largest percent change in any analysis was less than $2 \%$. The SSC USN position and velocity accuracies had a greater effect on the resulting RSO position accuracy, especially at the low and nominal error source levels.

One great benefit of the LinCov analysis tool is the ability to perform fast error budget analysis. This was performed for the baseline results as well as two ground station measurement periods in addition to the space sensor measurement periods scenarios. A consistent dominant error group is the ground station measurement bias and noise. In order to reduce the overall RSO RSS position accuracy, the ground station measurement bias and noise needs reduced. RSO process noise is dominant when the high error source level is used with the nominal tasking profile; this represents unknown dynamics effects on the 
RSO. For example, if the RSO has a high area-to-mass ratio the effect of SRP on the RSO orbit determination is dramatic. Better modeling of the RSO would be required in order to reduce the process noise.

Another great benefit of the LinCov analysis tool is the reduction in analysis time when compared to Monte Carlo analysis. One 50 sample Monte Carlo analysis run took approximately 4 hours to generate the results seen within this dissertation while one LinCov run created the results within minutes.

An interesting, consistent outcome throughout the results of this dissertation is that the high circular space sensor orbit and elliptical space sensor orbit had very similar results. This is beneficial in that the elliptical space sensor orbit has a shortened period than that of the high circular space sensor orbit. Therefore, it's possible to have more views of GEO objects with the elliptical space sensor orbit while achieving the same level of results of the high circular space sensor orbit.

The potential problem of the RSO falling outside the field of view of the ground station telescopes within the modeled two week period was not an item of concern for the given tasking scenarios. The field of view of the GEODSS ground station telescope is $1.23^{\circ} \mathrm{x}$ $1.61^{\mathrm{O}}$, which at geosynchronous range corresponds to about $860 \times 1125 \mathrm{~km}$ [8]. Only two scenarios break the field of view requirement: the ground station only results when only two ground station measurement periods are used, and the ground station plus low circular space sensor orbit scenario with only two ground station measurement periods, both with high error source levels.

The important application of the developed LinCov analysis tool is for design optimization, due to its efficiency and easily adaptable nature to different scenarios. This tool can be used to achieve a desired level of performance based on a variety of sensor specifications. This LinCov analysis tool is especially useful for the 808 geosynchronous satellites with available two line element data sets on the space track website [3].

\subsection{Future Work}

The LinCov analysis tool developed within this dissertation can be utilized to determine 
the accuracies achievable when other RSOs, in different geometries, are tracked. This would provide a more comprehensive picture of achievable GEO position accuracies dependent upon RSO orbital position.

Another area of future work is to investigate the effect of a constellation of space sensors on the results. It is clearly evident that a space based sensor improves RSO RSS position accuracies, so it would be helpful to determine if more space sensors improve the accuracies further, or provide the possibility of replacing some ground station measurement periods, and the potential of providing persistent 24 hour coverage.

The divergence that occurred when the high error source level of the RSO was set at $100 \mathrm{~km}$ downrange, $20 \mathrm{~km}$ altitude, and $20 \mathrm{~km}$ crosstrack should be investigated in more detail. This could help to determine if the EKF is adaptable and still the right tool to perform the analysis at this level, or if a different type of tool is required. If the EKF is adaptable to that level of error, the work still exists to see if it is possible to build a LinCov analysis tool from it. 


\section{REFERENCES}

[1] Vallado, D. A., Fundamentals of Astrodynamics and Applications, Microcosm Press, 4th ed., 2013.

[2] Curtis, H. D., Orbital mechanics for engineering students, Butterworth-Heinemann, 2nd ed., 2013.

[3] "space-track," https://www.space-track.org/auth/login, [Online Database] [retrieved 1 November 2015].

[4] Flohrer, T., "Classification of geosynchronous objects. Issue 15," Tech. Rep. GEN-DBLOG-00101-HSO-GR, ESA/ESOC, Darmstadt, Germany, 2013.

[5] Vallado, D. and Griesbach, J., "Simulating space surveillance networks," $A A S / A I A A$ Astrodynamics Specialist Conference Paper AAS 11-580, 2011.

[6] Sharma, J., Stokes, G. H., von Braun, C., Zollinger, G., and Wiseman, A. J., "Toward operational space-based space surveillance," Lincoln Laboratory Journal, Vol. 13, No. 2, 2002, pp. 309-334.

[7] "Ground-Based Electro-Optical Deep Space Surveillance," http:// www.afspc.af .mil/About-Us/Fact-Sheets/Display/Article/249016/ ground-based-electro-optical-deep-space-surveillance/, [online] [retrieved 10 May 2017].

[8] Bruck, R. F. and Copley, R. H., "GEODSS Present Configuration and Potential," Advanced Maui Optical and Space Surveillance Technologies Conference, 2014.

[9] Stokes, G. H., von Braun, C., Sridharan, R., Harrison, D., and Sharma, J., "The SpaceBased Visible Program," LINCOLN LABORATORY JOURNAL, Vol. 11, No. 2, 1998. 
[10] "Space Based Space Surveillance," http://www.afspc.af.mil/About-Us/ Fact-Sheets/Article/249017/space-based-space-surveillance-sbss/, $\quad 2017$, [online] [retrieved 10 May 2017].

[11] "U.S. Air Force Fact Sheet Geosynchronous Space Situational Awareness Program," $\quad$ http://www.afspc.af.mil/About-Us/Fact-Sheets/Article/730802/ geosynchronous-space-situational-awareness-program-gssap/, 2017, [online] [retrieved 10 May 2017].

[12] Vallado, D., Lowe, J., and Anderson, J., "Benefits of hosted payload architectures for improved GEO SSA," Advanced Maui Optical and Space Surveillance Technologies Conference, 2011.

[13] Lowe, J., Vallado, D., and Hall, B., "Technical analysis of commercially hosted optical payloads for enhanced SSA," Advanced Maui Optical and Space Surveillance Technologies Conference, 2010.

[14] Byrne, R., Griesmeyer, M., Schmidt, R., Shaddix, J., and Bodette, D., "Benefits of a Geosynchronous Orbit (GEO) Observation Point for Orbit Determination," Tech. rep., DTIC Document, 2011.

[15] Maybeck, P. S., Stochastic models, estimation, and control, Vol. 1, Academic press, 1982.

[16] Bhaskaran, S., Desai, S., Dumont, P., Kennedy, B., Null, G., Owen, W., Riedel, J., Synnott, S., and Werner, R., "Orbit determination performance evaluation of the deep space 1 autonomous navigation system," AIAA/AAS Space Flight Mechanics Meeting, Monterey, Citeseer, 1998.

[17] Christensen, R. S. and Geller, D., "Linear Covariance Techniques for Closed-Loop Guidance Navigation and Control System Design and Analysis," Journal of Aerospace Engineering, 2012. 
[18] Park, R., Cangahuala, L. A., Chodas, P., and Roundhill, I., "Covariance analysis of cassini titan flyby using sar and altimetry data," AIAA/AAS Astrodynamics Specialist Conference and Exhibit, 2006, p. 6398.

[19] Estefan, J. and Folkner, W., "Sensitivity of Planetary Cruise Navigation to Earth Orientation Calibration Errors," Telecommunications and Data Acquisition Progress Report, Vol. 123, 1995, pp. 1-29.

[20] Estefan, J. and Burkhart, P., "Enhanced Orbit Determination Filter Sensitivity Analysis: Error Budget Development," The Telecommunications and Data Acquisition Progress Report 42-11 6, pp. 24.

[21] Hicks, K. D. and Wiesel, W. E., "Autonomous orbit determination system for earth satellites," Journal of guidance, control, and dynamics, Vol. 15, No. 3, 1992, pp. 562566.

[22] Tombasco, J. and Axelrad, P., "Along-Track Separation Uncertainty Modeling Given Space-Based Optical Observations," Journal of Guidance, Control, and Dynamics, Vol. 35, No. 3, 2012, pp. 732-739.

[23] Tombasco, J., Orbit estimation of geosynchronous objects via ground-based and spacebased optical tracking, Ph.D. thesis, University of Colorado, 2011.

[24] Sharma, J., "Space-based visible space surveillance performance," Journal of Guidance, Control, and Dynamics, Vol. 23, No. 1, 2000, pp. 153-158.

[25] Faccenda, W. J., Ferris, D., Williams, C. M., and Brisnehan, D., "Deep Stare technical advancements and status," AMOS 2003 Technical Conference Proceedings (Advanced Maui Optical and Space Surveillance Technologies Conference), Maui Economic Development Board, Inc. Maui, HI, 2003.

[26] Braun, C. v., Sharma, J., and Gaposchkin, E. M., "Space-based visible metric accuracy," Journal of Guidance, Control, and Dynamics, Vol. 23, No. 1, 2000, pp. 175-181. 
[27] Montenbruck, O. and Gill, E., "Satellite orbits," Springer, Vol. 2, 2000, pp. 55.

[28] Mueller, A., "A Fast Recursive Algorithm for Calculating the Forces Due to the Geopotential (Program: GEOPOT)," Tech. rep., JSC Internal Note 75-FM-42 (JSC-09731), NASA, 1975.

[29] Roithmayr, C. M., "Contributions of spherical harmonics to magnetic and gravitational fields," Tech. Rep. NASA/TM-2004-213007, L-18358, NASA Langley Research Center, Hampton, VA, 2004.

[30] Lear, W. M., "Kalman Filtering Techniques," Tech. Rep. JSC-20688, NASA Johnson Space Center, Houston,Texas, 1985.

[31] "Proceedings of the 2000 Space Control Conference," Tech. Rep. STK-255, Massachusetts Institute of Technology Lexington Lincoln Lab, 2000.

[32] Schildknecht, T., Früh, C., Herzog, A., Hinze, J., and Vananti, A., "AIUB efforts to survey, track, and characterize small-size objects at high altitudes," Proceedings of 2010 AMOS Technical Conference, 2010, pp. 14-17.

[33] "Cubesat design specification rev. 13," Tech. rep., Cal Poly San Luis Obispo, 2014.

[34] Kreyszig, E., Advanced Engineering Mathematics, John Wiley \& Sons, eighth ed., 1999.

[35] Chapel, J., Stancliffe, D., Bevacqua, T., Winkler, S., Clapp, B., Rood, T., Gaylor, D., Freesland, D., and Krimchansky, A., "Guidance, Navigation, and Control Performance for the GOES-R Spacecraft," Tech. Rep. GSFC-E-DAA-TN19917, NASA Goddard Space Flight Center; Greenbelt, MD, 2014.

[36] Abbot, R. I., Gaposchkin, E. M., and Braun, C. v., "Midcourse Space Experiment Precision Ephemeris," Journal of Guidance, Control, and Dynamics, Vol. 23, No. 1, 2000, pp. 186-190. 
[37] "Swedish Space Corporation Universal Space Network," http://www . sscspace.com/Products-Services/satellitemanagementservices/ ground-network-prioranet-1, [online] [retrieved 10 March 2017]. 


\section{CURRICULUM VITAE}

Blythe Andrews

\section{Education}

Ph.D. $\quad$ Aerospace Engineering, Utah State University, Logan, Utah, 2017.

M.S. Astronautical Engineering, Air Force Institute of Technology, WrightPatterson AFB, Ohio, 2012.

B.S. Mechanical Engineering, University of Pittsburgh, Pittsburgh, Pennsylvania, 2006.

\section{Work Experience}

2013-2014 Executive Officer and Instructor of Astronautics, United States Air Force Academy, Colorado Springs, CO

Administrates day to day operations for 34 member department, course directs Introduction to Astronautics for 490 cadets, responsible for all course material and manages 12 instructors, teaches Introduction to Astronautics to 35 cadets.

2012-2013 Instructor of Astronautics, United States Air Force Academy, Colorado Springs, $\mathrm{CO}$

Taught Introduction to Astronautics to 85 cadets, chosen as new instructor of the year by peers.

2009-2010 Executive Officer, Defense Contract Management Agency, Joint Base Balad, Iraq

Oversaw operations for Defense Contract Management Agency Northern Iraq 
in support of Operation Iraqi Freedom, led 4 member team, responsible for logistical support for 40 personnel located at 18 Forward Operating Bases throughout Iraq.

2008-2010 Test Director, National Hover Test Facility, Edwards AFB, CA Directed tests for \$100M Missile Defense Agency National Hover Test Facility, Directed 38 member team testing Multiple Kill Vehicle (MKV), Directorate Team Trophy Winner.

2007-2008 Deputy Branch Chief - Liquid Rocket Engines, Air Force Research Laboratory, Edwards AFB, CA Oversaw \$32M/yr US liquid rocket engine science \& technology budget, led 20 members working 29 R $6 D$ programs, led $\$ 3 M$ rocket engine health management program acquisition.

2006-2007 Rocket Propulsion Program Manager, Air Force Research Laboratory, Edwards AFB, CA

Directed $\$ 750 K$ engine program for engine health management, chaired DoD/industry Rocket Engine Health Management conference. 